\title{
Comparative study of catalase-peroxidases (KatGs)
}

\author{
by \\ Rahul Singh \\ A thesis \\ submitted to the Faculty of Graduate Studies \\ in partial fulfillment of the requirements for the degree of \\ DOCTOR OF PHILOSOPHY
}

Department of Microbiology

University of Manitoba

Winnipeg, Manitoba

(C) Rahul Singh, August 2006 
THE UNIVERSITY OF MANITOBA

FACULTY OF GRADUATE STUDIES

COPYRIGHT PERMISSION

Comparative study of catalase-peroxidases (KatGs)

BY

Rahul Singh

A Thesis/Practicum submitted to the Faculty of Graduate Studies of The University of Manitoba in partial fulfillment of the requirement of the degree

OF

DOCTOR OF PHILOSOPHY

Rahul Singh $\mathbb{C} 2006$

Permission has been granted to the Library of the University of Manitoba to lend or sell copies of this thesis/practicum, to the National Library of Canada to microfilm this thesis and to lend or sell copies of the film, and to University Microfilms Inc. to publish an abstract of this thesis/practicum.

This reproduction or copy of this thesis has been made available by authority of the copyright owner solely for the purpose of private study and research, and may only be reproduced and copied as permitted by copyright laws or with express written authorization from the copyright owner. 
Dedicated to my mother

Banmala Singh (1951-2005) 


\begin{abstract}
Catalase-peroxidases (KatGs) act as a protective enzyme against oxidative stress
\end{abstract} during the exponential phase of growth. KatG from $M$. tuberculosis is better known for its role in the activation of isoniazid (INH), a front line anti-tuberculosis prodrug, through it's conversion to isonicotinoyl-NAD adduct, which inhibits the mycolic acid synthesis. Biochemical characterization of KatGs from Burkholderia pseudomallei, Mycobacterium tuberculosis, Escherichia coli, Bacillus stearothermophilus, Archaeoglobus fulgidus, Synechosystis PCC 6803, and Rhodobacter capsulatus, was carried out to compare their enzyme kinetics in view of the recent success in crystallization of the KatGs. Catalaseperoxidases show substantial catalase specific activity with significant variation among the seven KatGs studied. A large variation was also evident in the peroxidase activity of the seven KatGs. Differences in the $K_{\mathrm{m}}$ values for both catalase and peroxidase reactions are indicative of the subtle differences in the protein structure, particularly in the vicinity of active site heme and the access channel for the substrate. The role of catalaseperoxidases in the activation of $\mathrm{NH}$ was also characterized and compared using NADH and some other pyridine derivatives as substrate. KatGs catalyze the oxidation of NADH to form $\mathrm{NAD}^{+}$and either $\mathrm{H}_{2} \mathrm{O}_{2}$ or superoxide radical depending on $\mathrm{pH}$. The $\mathrm{NADH}$ oxidase reaction requires molecular oxygen, does not require hydrogen peroxide, is not inhibited by superoxide dismutase or catalase, and has a $\mathrm{pH}$ optimum of 8.75 , clearly differentiating it from the peroxidase and catalase reactions with $\mathrm{pH}$ optima of 5.5 and 6.5 , respectively, and from the NADH peroxidase-oxidase reaction of horseradish peroxidase. $B$. pseudomalle $\mathrm{KatG}$ has a relatively high affinity for $\mathrm{NADH}\left(K_{\mathrm{m}}=12 \mu \mathrm{M}\right)$, but the oxidase reaction is slow $\left(k_{\mathrm{cat}}=0.54 \mathrm{~min}^{-1}\right)$ compared with the peroxidase and 
catalase reactions. The catalase-peroxidases also catalyze the hydrazinolysis of isonicotinic acid hydrazide (INH) in an oxygen- and $\mathrm{H}_{2} \mathrm{O}_{2}$-independent reaction, and KatG-dependent radical generation from a mixture of NADH and INH is two to three times faster than the combined rates of separate reactions with $\mathrm{NADH}$ and INH alone. The major products from the coupled reaction, identified by HPLC fractionation and mass spectrometry, are $\mathrm{NAD}^{+}$and isonicotinoyl-NAD. Isonicotinoyl-NAD synthesis from a mixture of $\mathrm{NAD}^{+}$and $\mathrm{NH}$ is KatG-dependent and is activated by manganese ion. M. tuberculosis KatG catalyzes isonicotinoyl-NAD formation from $\mathrm{NAD}^{+}$and INH more efficiently than B. pseudomallei KatG. A similar comparison of Ser315Thr and Ser315Asn variants of $\mathrm{MtKatG}$, the two most common mutations occurring in the kat $G$ gene among drug resistant strains of $M$. tuberculosis, demonstrates that variants have lower rates of hydrazinolysis and isonicotinoyl-NAD synthase activity and undetectable NADH oxidase activity while maintaining substantial catalase and peroxidase activities. These properties are consistent with the enzyme being required for the maintenance of virulence while developing resistance against INH. 


\section{ACKNOWLEDGEMENTS}

I am deeply indebted to my advisor, Dr. Peter Loewen, for his expert guidance and faith in my abilities throughout this project. His financial and personal support during my studies is very much appreciated. His thoughtfulness and dedication towards his research always remained a source of inspiration. I am also thankful for the guidance and encouragement I received from my advisory committee members, Dr. E. Worobec, Dr. J. O’Neil and especially Dr. I. J. Oresnik for his critical analysis of my progress. I am also very grateful to Mr. Jacek Switala for his help and cooperation with his excellent technical expertise. I would also like to thank Dr. Lynda Donald, Dr. Maureen Spearman, Dr. Linda Cameron, and Mr. Norm Huzel for their assistance. I greatly appreciate Ms. Sharon Berg for the unreserved support I have received countless time from her. Our collaborators in Spain (Prof Ignacio Fita's lab) and France (Dr. Anabella Ivancich's Lab) are greatly appreciated. A special thank goes to the Loewen lab members; Taweewat, Ben, Amarbeer and Vikas and the past members Prashen and Sherif. I would also thank to the faculty members and support staff in the department of microbiology for their cooperation and support. My fellow graduate students are greatly appreciated for their friendship and encouragement with special mention of Ayush, Patrick, Matt, Taylor, and many more. Finally I owe a great deal to my father, my sister Priyanka, her husband Avnish, my friends, Gurusharan and Ajay, for always been there for me, and my wife Tejomayee who deserves a very special appreciation for the patience, understanding and tremendous support she has continually provided while I was writing this thesis. 


\section{TABLE OF CONTENTS}

\section{Page}

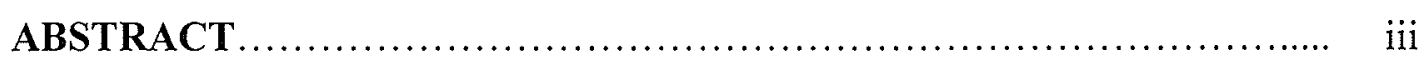

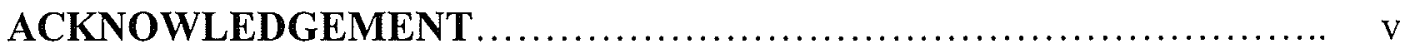

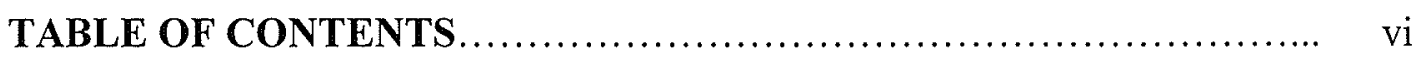

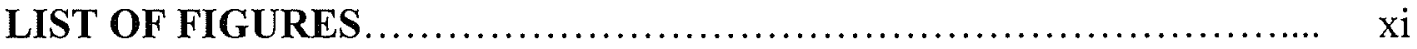

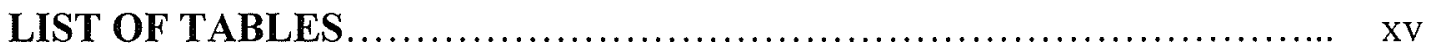

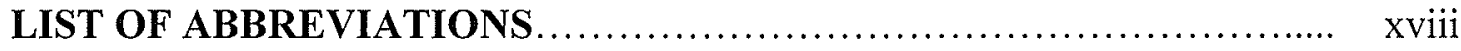

1. GENERAL INTRODUCTION............................... 1

1.1. Oxygen: Elixir of life - and death................................. 1

1.2. The oxygen molecule............................................... 4

1.2. The oxygen molecule............................................... 8

1.3. Antioxidant Systems........................................... 6

1.3.1. Non-Enzymatic Defenses........................................ 7

1.3.2. Enzymatic Defenses.............................................. 8

1.4. The nonfunctional, heme containing catalases......................... 11

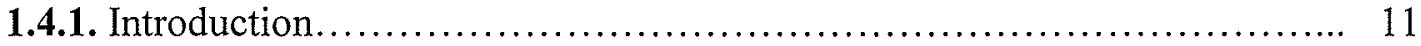

1.4.2. Structural properties of catalases.................................. 13

1.4.3. An electrical potential field in the channel of mono-functional catalases... 15

1.5. Non-heme or manganese containing catalases.......................... 16

1.6. Monofunctional peroxidases......................................... 16

1.7. Catalase-peroxidases........................................... 19

1.7.1. Phylogeney of catalase-peroxidases................................ 19

1.7.2. Regulation of catalase-peroxidase gene $(k a t G)$ expression................ 21

1.7.3. Structure and mechanism of catalase-peroxidases....................... 23

1.7.3.1. The unusual covalent modifications............................... 29

1.7.3.1.1. A covalent adduct structure linked Trp111-Tyr238-Met264.......... 31

1.7.3.1.2. Perhydroxy modification of Heme.............................. 35

1.7.3.1.3. Perhydroxy modification on the Indole of $\operatorname{Trp} 111 \ldots \ldots \ldots \ldots \ldots \ldots \ldots . . . \ldots 5$

1.7.3.2. Three large loops in the catalytic part of KatGs....................... 36

1.7.3.3. Substrate access channel.......................................... 37

1.7.3.4. $\mathrm{pH}$ induced changes in catalase-peroxidases ......................... 41

1.7.4. Protein radicals in catalase-peroxidases............................... 43

1.7.5. Catalasae-peroxidase from different organisms........................ 45

1.7.5.1. Catalase-peroxidase of Burkholderia pseudomallei (BpKatG)............ 45 
1.7.5.2. Catalase-peroxidase of Mycobacterium tuberculosis (MtKatG).......... 47

1.7.5.3. Catalase-peroxidase of Escherichia coli (EcKatG)...................... 48

1.7.5.4. Catalase-peroxidase of Synechocystis PCC 6803 (SyKatG)............... 49

1.7.5.5. Catalase-peroxidase of Archaeoglobus fulgidus (AfKatG) ................ 50

1.7.5.6. Catalase-peroxidase of Bacillus stearothermopohilus (BsKatG).............. 50

1.7.5.7. Catalase-peroxidase of Rhodobacter capsulatus (RcKatG)............... 51

1.7.6. Catalase-peroxidase and isoniazid activation............................ 51

1.7.6.1. KatG mutations involve in isoniazid resistance....................... 53

1.7.6.2. Analysis of potential isoniazid binding site......................... 57

1.8. Objective of the thesis................................................... 58

2. MATERIALS AND METHODS........................................ 59

2.1. Escherichia coli strains, plasmids and bacteriophage ...................... 59

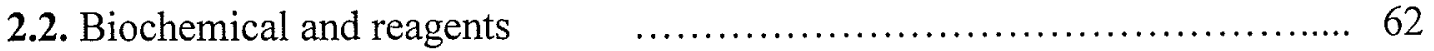

2.3. Media, growth conditions and storage of cultures......................... 62

2.4. DNA manipulation.................................................... 63

2.4.1. Preparation of synthetic oligonucleotides.............................. 63

2.4.2. Site-directed mutagenesis strategy.................................... 64

2.4.3. Reconstruction of $M$. tuberculosis kat $G$ subclones with desired mutation... 80

2.4.4. Reconstruction of B. pseudomallei kat $G$ subclones with desired mutation... 80

2.4.5. DNA isolation and purification..................................... 81

2.4.6. Restriction endonuclease digestion of DNA ........................... 82

2.4.7. Agarose gel electrophorosis......................................... 82

2.4.8. Ligation ............................................................... 83

2.4.9. Transformation........................................................ 83

2.4.10. DNA sequencing ..................................................... 84

2.5. Purification of catalase-peroxidases................................... 85

2.6. Polyacrylamide gel electrophoresis (PAGE) of proteins and staining........ 87

2.7. Enzyme assay and protein quantitation................................. 89

2.7.1. Catalase activity .................................................... 89

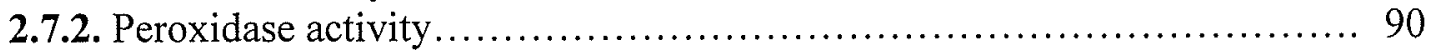

2.7.3. INH hydrazine lyase activity ............................................ 91

2.7.4. NADH oxidation.................................................... 92

2.7.5. Isonicotinoyl $N A D$ synthase activity ...................................... 92

2.7.6. Protein Concentration................................................. 93

2.8. Absorption spectrophotometry of KatGs.................................. 93

2.9. Effects of inhibitors................................................. 93

2.10. High Performance Liquid Chromatography characterization of reaction products........................................................................ 94

2.11. Mass Spectrometry ............................................... 95 


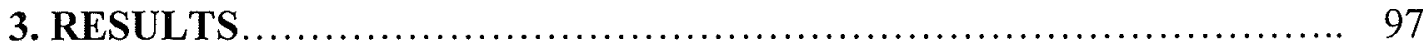

3.1 Introduction............................................................. 97

3.2. Purification and characterization of $B p K a t G, M t K a t G, E c K a t G, S y K a t G$,

BsKatG, AfKatG and,RcKatG....................................... 98

3.2.1 Purification of KatGs................................................ 98

3.2.2 Polyacrylamide gel electrophoresis (PAGE) of the catalase-peroxidases.... 102

3.2.3. UV-visible absorption spectroscopy of catalase-peroxidases................. 109

3.2.4. $\mathrm{pH}$ profile for the catalase and peroxidase activities........................ 109

3.2.5. Specific catalase and peroxidase activities of KatGs...................... 116

3.2.6. Kinetic characterization of KatGs....................................... 116

3.2.7. Effect of heme inhibitor on catalase activity of KatGs...................... 130

3.3. KatGs and isoniazid activation...................................... 134

3.3.1. Catalase-peroxidases (KatG) exhibit NADH oxidase activity............... 135

3.3.1.1. NADH oxidation by KatG.......................................... 136

3.3.1.2 Visualization of NADH oxidase activity by PAGE under non-denaturating conditions............................................. 143

3.3.1.3. Kinetic characterization of NADH oxidase activity ..................... 147

3.3.1.4. Comparison of NADH oxidase activity with NADH peroxidase-oxidase reaction by HRP...................................... 147

3.3.1.5. Effect of NADH on the peroxidase activity of KatGs..................... 153

3.3.2. INH hydrazine lyase activity by KatGs.................................... 153

3.3.3. Interaction of KatG with a mixture of $\mathrm{INH}$ and NADH.................... 158

3.3.3.1. $\mathrm{NAD}^{+}$as Precursor for Isonicotinoyl-NAD ............................. 168

3.3.3.2. Comparison of the rate of isonicotinoyl-NAD synthase activity by KatGs. 168

3.3.5. NADH oxidation can be uncoupled from superoxide Formation................ 173

3.4. Resistance against isoniazid in Mycobacterium tuberculosis.................. 175

3.4.1. Introduction................................................... 175

3.4.2. Purification and characterization of Ser $315 \mathrm{Thr}$, Ser 315 Asn variants of $M$. tuberculosis catalase-peroxidase......................................... 176

3.4.3. Catalase and peroxidase specific activities of MtKatG and variants......... 177

3.4.4. Kinetic characterization of Ser315Thr and Ser315Asn variants............. 177

3.4.5. Comparasion of heme inhibition by cyanide and azide..................... 182

3.4.6. Effect of Ser315 mutation on oxidase activity and isoniazid activation........ 182

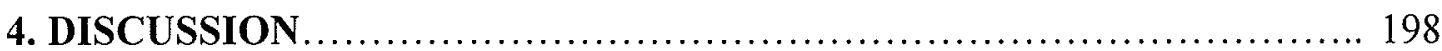

4.1. Location of conserved residues in catalase-peroxidases..................... 198

4.2. Overview and comparison of catalase and peroxidase activities................ 201

4.3. The oxidase, lyase and synthase activities of KatGs......................... 203

4.4. Modulation of isoniazid activation by Ser315 variants in MtKatG............. 215

4.5. Future directions.................................................... 216

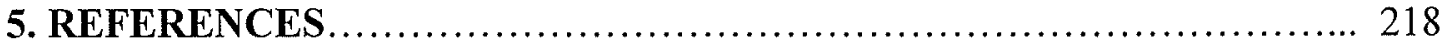




\section{LIST OF FIGURES}

Figure

Page

1.1. Schematic representation of intermediates between water and oxygen........ 2

1.2. Molecular orbital diagrams of molecular oxygen, superoxide, hydrogen peroxide, and singlet oxygen................................................. 5

1.3. Structures of BpKatG, MtKatG, HmkatG and $\mathrm{SyKatG} \ldots \ldots \ldots \ldots \ldots \ldots \ldots . \ldots 24$

1.4. Structures of $\mathrm{N}$-terminal of $\mathrm{BpKatG}$, cytochrome $c$ Peroxidase, single subunit of horseradish peroxidase, and single subunit of ascorbate peroxidase.......... 25

1.5. The active site residues in the proximal and distal side of BpKatG and overlay of cytochrome $c$ peroxidase with $\mathrm{N}$-terminal of BpKatG............. 28

1.6. Structural representation of covalent modifications in $B p K a t G \ldots \ldots \ldots \ldots \ldots . . . .30$

1.7. Structures of INH, NADH, isonicotinoyl-NAD adduct and isonicotinoyl-NAD adduct in bound form with InhA protein of $M$. tuberculosis...................... 54

2.1. Simplified restriction map of the chromosomal insert containing the M. tuberculosis kat $G$ gene in plasmids pAH1, pMt-EC and pMtG-CX ........ 65

2.2. General reconstruction protocol used for the generation of variant MtKatG's from mutagenized subclone I for protein expression................................... 66

2.3. The DNA sequence and corresponding amino acid sequence of M. tuberculosis showing the restriction sites and the target codones selected for mutagenesis in this study....

2.4. Simplified restriction map of the chromosomal insert containing the B. pseudomallei kat $G$ gene in plasmids $\mathrm{pBpG}$ and $\mathrm{pBpG}-\mathrm{CH}$..

2.5. General reconstruction protocol used for the generation of variant BpKatG's from mutagenized subclone I for protein expression.

2.6. The DNA sequence and corresponding amino acid sequence of $B$. pseudomallei showing the restriction sites and the target codones selected for mutagenesis in this study.

3.2.1. SDS-polyacrylamide analysis of purified KatGs...................... 103

3.2.2. Absorption spectra of KatGs..................................... 105

3.2.3. $\mathrm{pH}$ profile for catalase and peroxidase activities of KatGs................ 111

3.2.4. Effect of $\mathrm{H}_{2} \mathrm{O}_{2}$ concentration on the initial catalatic velocities of KatGs.... 118

3.2.5. Effect of ABTS concentration on the initial peroxidatic velocities of KatGs........................................................ 122

3.2.6. Effect of $\mathrm{H}_{2} \mathrm{O}_{2}$ concentration on the initial peroxidatic velocities of KatGs............................................................. 126

3.2.7. Comparison of sensitivity of KatGs to cyanide $(\mathrm{KCN})$ or azide $\left(\mathrm{NaN}_{3}\right) \ldots \quad 131$

3.3.1. NADH oxidation by catalase-peroxidases............................. 138

3.3.2. Migration of purified BpKatG on a $8 \%$ SDS-polyacrylamide gel and a nondenaturing $8 \%$ polyacrylamide gel.

3.3.3. $\mathrm{pH}$ dependence of $\mathrm{NADH}$ oxidation by $\mathrm{BpKatG}$ determined by radical formation at $560 \mathrm{~nm}$

3.3.4. Elution profiles from reverse phase HPLC of reaction products from mixtures containing $\mathrm{NADH}$ and $\mathrm{KatG}$. 
3.3.5. Effect of NADH concentration on the initial NADH oxidase velocities

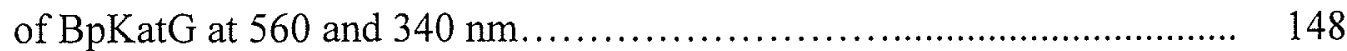

3.3.6. Effects of $\mathrm{NAD}^{+}$and Pyridoxine on NADH oxidase activity............... 149

3.3.7. Removal of hydrazine form isoniazid ............................... 154

3.3.8. Effect of manganese on the removal of hydrazine form isoniazid........... 155

3.3.9. Synergistic effect of $I N H$ and $\mathrm{NADH}$ on radical generation in a solution containing INH, NADH and KatG.

3.3.10. Elution profiles from reverse phase HPLC of reaction products from mixtures containing INH, NADH and KatGs.

3.3.11. Elution profiles from reverse phase HPLC of reaction products from mixtures containing INH, $\mathrm{NADH}, \mathrm{Mn}^{2+}$ and KatGs

3.3.12. Part of a matrix-assisted laser desorption ionization mass spectrum of a reaction mixture after $18 \mathrm{~h}$ of incubation showing the isonicotinoyl-NAD product ion.

3.3.13. Elution profiles from reverse phase HPLC of reaction products from mixtures containing $\mathrm{INH}, \mathrm{NAD}^{+}, \mathrm{Mn}^{2+}$ and KatGs.

3.3.14. Rate of synthesis of the isonicotinoyl-NAD adduct.

3.4.1. SDS-polyacrylamide analysis of purified $\mathrm{MtKatG}$ and variants Ser315Thr and Ser315Asn

3.4.2. Absorption spectra of MtKatG and variants Ser315Thr and Ser315Asn..... 179

3.4.3. Effect of $\mathrm{H}_{2} \mathrm{O}_{2}$ concentration on the initial catalatic velocities of Ser315Thr and Ser315Asn variants of MtKatG.

3.4.4. Effect of ABTS concentration on the initial catalatic velocities of the Ser315Thr and Ser315Asn variants of MtKatG

3.4.5. Effect of $\mathrm{H}_{2} \mathrm{O}_{2}$ concentration on the initial peroxidatic velocities of Ser315Thr and Ser315Asn variants of MtkatG.

3.4.6. Comparison of the sensitivity of $\mathrm{MtKatG}$ and its variants to cyanide $(\mathrm{KCN})$ and azide $\left(\mathrm{NaN}_{3}\right)$.....

3.4.7. NADH oxidation by MtKatG and variants Ser315Thr and Ser315Asn..... 190

3.4.8. Removal of hydrazine form isoniazid by MtKatG and variants............ 192

3.4.9. Effect of NADH on the hydrazine lyase activity by MtKatG and variants $a$, Isonicotinoyl-NAD synthase activity by MtKatG and variants $b$................ 194

4.1. Location of residues in BpKatG that are identical in more than $95 \%$ of the 53 catalase-peroxidase sequences available

4.2. Amino acid sequence alignment of BpKatG, MtKatG, EcKatG, SykatG, BskatG, AfkatG, RcKatG

4.3. Scheme showing the relationship of the five activities of KatG 


\section{LIST OF TABLES}

Table

Page

2.1. Genotypes and sources of $E$. coli strains, plasmids and bacteriophage used in this study....

2.2a. Sequence of oligonucleotides and restriction fragments used for the site directed mutagenesis of $M$. tuberculosis kat $G$.

61

2.2b. Sequence of oligonucleotides and restriction fragments used for the site directed mutagenesis of $B$. pseudomallei kat $G$.

2.2c. Primers used for the polymerase chain reaction for the cloning of kat $G$ from $M$. tuberculosis into $\mathrm{pET} 28 \mathrm{~b}+$ vactor..

3.2.1. Comparison of the effect of supplementing growth medium with $\mathrm{FeCl}_{3}$, $\delta$ - aminolevulinic acid (ALA) and hemen on the expression of catalase activity of $\mathrm{MtKatG}$

3.2.2a. Purification of wild type BpKatG and MtKatG from E. coli strain UM262 harboring plasmid encoded kat $G$ of B. pseudomallei and $M$. tuberculosis.

3.2.2b. Purification of wild type EcKatG and AfKatG from E. coli strain UM262 harboring plasmid encoded kat $G$ of E. coli and A. fulgidus

3.2.3. Comparison of observed optical absorbance maxima, $A_{407 / 280}$ ratio and heme/subunit ratio for purified KatGs

3.2.4. $\mathrm{pH}$ optima for catalatic and peroxidatic activities of KatG..................... 110

3.2.5. Comparison of catalase and peroxidase activities of KatGs................... 117

3.2.6. Comparison of the observed catalatic kinetic parameters of purified KatGs, using $\mathrm{H}_{2} \mathrm{O}_{2}$ as substrate.

3.2.7. Comparison of the observed peroxidatic kinetic parameters of purified

KatGs, using ABTS as substrate.

3.2.8. Comparison of the observed peroxidatic kinetic parameters of purified KatGs, using $\mathrm{H}_{2} \mathrm{O}_{2}$ as substrate for the compound I formation

3.2.9. Comparison of sensitivity of $\mathrm{KatGs}$ to cyanide $(\mathrm{KCN})$ or azide $\left(\mathrm{NaN}_{3}\right) \ldots \ldots 132$

3.3.1. Comparison of NADH oxidase activity of KatGs.......................... 137

3.3.2. KatG-mediated radical production in the presence of different substrates..... 142

3.3.3. Comparison of kinetic constants for $\mathrm{NADH}$ oxidase, catalase and, peroxidase activities of BpKatG.

3.3.4. Comparison of NADH oxidation by HRP, BpKatG, and the W111F.

3.3.5. Effect of $\mathrm{NADH}$ or $\mathrm{NAD}^{+}$on the peroxidase activity of BpKatG, MtKatG and Eckat $G$ using ABTS or $o$-dianisidine

3.3.6. Comparison of effect of $\mathrm{MnCl}_{2}$ and $\mathrm{NADH}$ on the hydrazine lyase activity of catalase-peroxidases.

3.3.7. Comparison of expected and observed $\mathrm{m} / \mathrm{z}$ values of ions in the MS/MS spectra of NADH and the product of the reaction of KatG with INH and $\mathrm{NADH}$ identified as isonicotinoyl-NAD.

3.3.8. Comparison of catalase, peroxidase, and oxidase activities of $\mathrm{BpKatG}$ and its variants 
3.4.1. Comparison of observed optical absorbance maxima, $A 407 / 280$ ratio and heme/subunit ratio for purified KatGs.

3.4.2. Comparison of catalase and peroxidase activities of MtKatGs and the Ser315Thr and Ser315Asn variants.

3.4.3. Comparison of the observed catalatic kinetic parameters of purified MtKatG and the Ser315Thr and Ser315Asn variants using $\mathrm{H}_{2} \mathrm{O}_{2}$ as substrate.

3.4.4. Comparison of the observed peroxidatic kinetic parameters of purified $\mathrm{MtKatG}$ and the Ser315Thr and Ser315Asn variants using ABTS as substrate

3.4.5. Comparison of the sensitivity of $M t K a t G$ and its variants to cyanide $(\mathrm{KCN})$ and azide $\left(\mathrm{NaN}_{3}\right)$.

3.4.6. Comparison of NADH oxidase activity, hydrazine lyase, and synthase activity by $\mathrm{MtKatG}$ and variants.

4.1. Comparison of the percentage of highly conserved residues ( $>95 \%$ identical) in KatGs compared to other families of proteins. 200 


\section{List of Abbreviations}

A

ABTS

$\mathrm{Amp}^{\mathrm{R}}$

APX

AfKatG

bp

BpKatG

BLC

BsKatG

$\mathrm{CcP}$

$\mathrm{Ci}$

CIP

Da

DEAE

DMSO

DNA

dNTP

$\mathrm{e}^{-}$

EcKatG

EDTA

$\mathrm{H}^{+}$

$\mathrm{HmKatG}$

$\mathrm{H}_{2} \mathrm{O}$

$\mathrm{H}_{2} \mathrm{O}_{2}$

HPI

HPII

HRP

INH

KatG

$\mathrm{Kbp}$

$k_{\text {cat }}$

$\mathrm{kDa}$

$K_{\mathrm{m}}$

LB

$\mu \mathrm{M}$

$\mathrm{M}$

$\mathrm{mg}$

$\mathrm{mL}$

$\mathrm{mM}$

MtKatG

$\mathrm{NADH}$

NADPH

$\mathrm{NAD}^{+}$

NBT
Absorbance

2,2'-azinobis(3-ethylbenzothiazolinesulfonic acid)

Ampicillin resistance

Ascorbate peroxidase

KatG from Archeoglobus fulgidus

Base pairs (s)

KatG from Burkholderia pseudomallei

Bovine liver catalase

KatG from Bacillus stearotthermophilus

Cytochrome $c$ peroxidase

Curie

calf intestinal alkaline phosphatase

Daltons(s)

Diethylaminoethyl

DImethylsulfoxide

Deoxyribonucleic acid

Deoxyribonucleoside triphosphate

Electron

KatG from Escherichia coli

Ethylenediaminetetraacetic acid

Proton

KatG from Haloarcula marismortui

Water

Hydrogen peroxide

Hydroperoxidase I from Escherichia coli

Hydroperoxidae II from Escherichia coli

Horseradish peroxidase

Isonicotinic acid hydrazide (isoniazid)

Catalase-peroxidase

Kilo base pairs(s)

Turnover number

Kilo Daltons

Michealis-Menten constant

Lauria-Bertani

Micromolar

Molarity/molar

Milligrams

Milliliter

Millimolar

KatG from Mycobacterium tuberculosis

Nicotinamide adenine dinucleotide (reduced)

Nicotinamide adenine dinucleotide phosphate

Nicotinamide adenine dinucleotide (oxidized)

Nitroblue Tetrazolium 
$\mathrm{nm}$

RcKatG

SyKatG

OD

PAGE

PEG

PMSF

RNAse

ROS

SDS

Tris

$\mathrm{V}$

$\mathrm{V}_{\max }$

$\mathrm{w} / \mathrm{v}$

$w / w$
Nanometers(s)

KatG from Rhodobacter capsulatis

KatG from Synechocystis

Optical density

Polyacrylamide gel electrophoresis

Polyethylene glycol

Phenylmethylsulfonyl fluoride

Ribonuclease

Reactive oxygen species

Sodium dodecyl sulfate

Tris (hydroxymethyl) aminomethane

volts

Maximum velocity

Weight per volume

Weight per weight 


\section{General Introduction}

\subsection{Oxygen: Elixir of life - and death}

Although the story of oxygen $\left(\mathrm{O}_{2}\right)$ began with the discoveries of the British theologian J. B. Priestley, the French chemist A. L. Lavoisier, and the Swedish pharmacist C.W. Scheele in the eighteenth century, the concept of $\mathrm{O}_{2}$ as a requirement for aerobic life existed even in ancient civilization and history is full of such examples referring to the significance of oxygen; for example, the Sanskrit word "prana vayu" which means "the gas of life" appears frequently in the Vedas, the sacred writings of ancient Indian civilization. Another example came from 1604, when the Polish alchemist Michael Sendivogius wrote that "Man was created of the earth, and lives by virtue of the air; for there is in the air a secret food of life... whose invisible congealed spirit is better than the whole Earth" and proposes that this 'aerial food of life' circulated between the air and earth by way of an unusual salt — nitre, or saltpeter. He evidently believed that he had discovered the Elixir of life, "without which no mortal can live, and without which nothing grows or is generated in the world" (Lane, 2002). Thus, for centuries considered critical to life itself, $\mathrm{O}_{2}$ remains, and will always remain one of our essential elements. However, by the end of twentieth century, $\mathrm{O}_{2}$ had become the focus of controversy, resulting from observations that it can be dangerous, toxic and deleterious to biological systems (Doroshow, 1989).

During the past 30 years, the fields of free radical chemistry and biology have risen from relative obscurity to become mainstream elements of biomedical investigation 


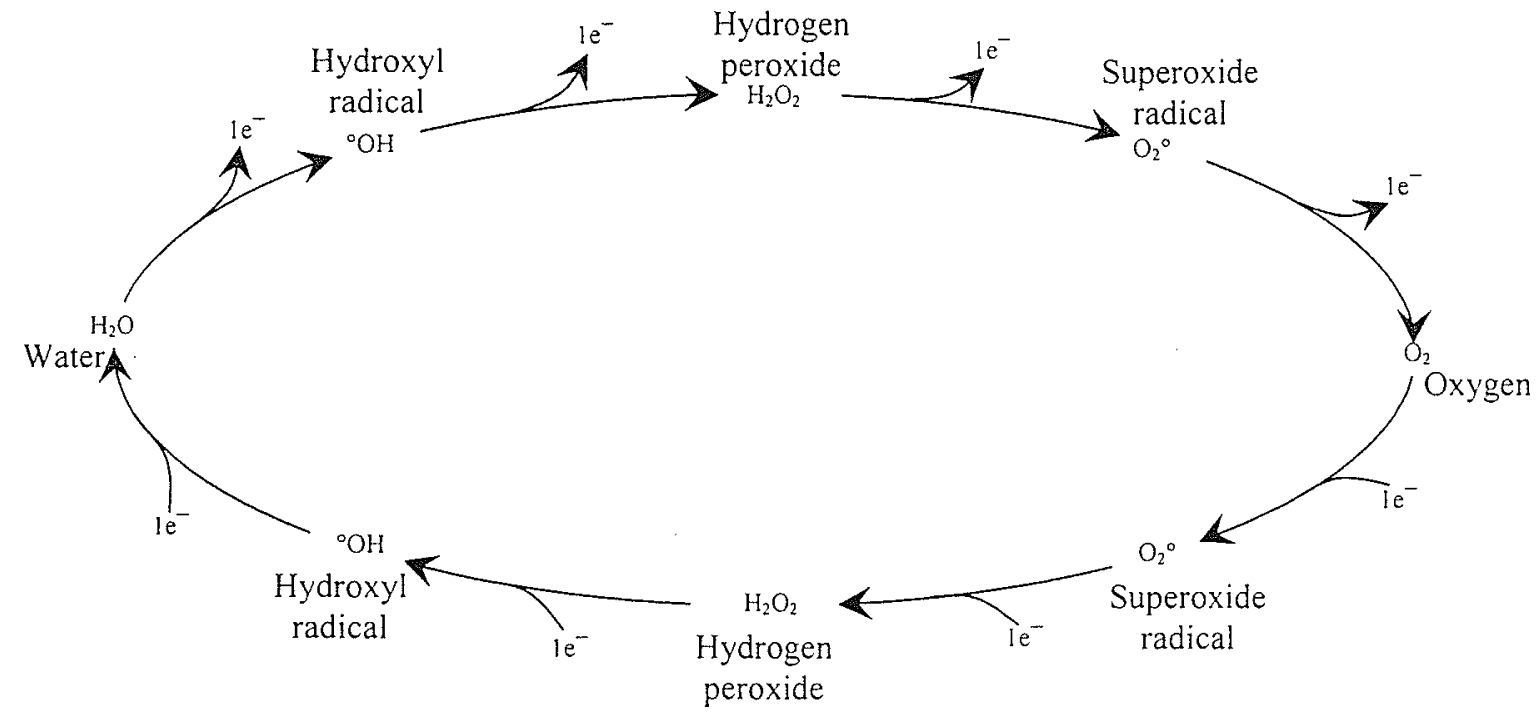

Figure 1.1. Schematic representation of intermediates between water and oxygen. Only changes in number of electron $\left(\mathrm{e}^{-}\right)$is shown in each direction. 
and pharmaceutical development. There is increasing evidence that oxidative stress, defined as an imbalance between oxidants and antioxidants in favor of the former, leads to detrimental biochemical reactions between $\mathrm{O}_{2}$ and other molecules within both the human body and the environment, which generate reactive species of oxygen better known as reactive oxygen species (ROS). During evolution nature selected $\mathrm{O}_{2}$ as the primary electron acceptor in the most efficient mechanisms for producing usable biological energy. With the use of $\mathrm{O}_{2}$ as a terminal oxidant came the formation of the partially reduced oxygen species: the superoxide anion, hydrogen peroxide, and the hydroxyl radical which are too reactive to be tolerated within living systems. These reactive oxygen species are an important contributing factor in several chronic human diseases, including atherosclerosis and related vascular diseases, mutagenesis and cancer, neurodegeneration, immunologic disorders, and the aging process (Doroshow, 1989, Harman, 1986, Rice-Evans, 1988). The first direct proof in our understanding that oxygen free radicals mediated oxygen toxicity appeared in 1954, when Rebeca Gerschman, Daniel Gilbert, and colleagues noted that the pattern of X-radiation damage to lung tissue was similar to that induced by high oxygen concentrations (Greshman et al., 1954). Apart from showing the cumulative effects of hyperoxia and $\mathrm{x}$-irradiation, they also reported that many chemical agents affording protection against the toxic effect of radiation also protected against oxygen poisoning. The essential feature of their theory was that both oxygen poisoning and the biological effects of $\mathrm{x}$-irradiation share a common mechanism of action, specifically the reactions of free radicals, which is mediated by exactly the same fleeting intermediates which can be produced either from oxygen or water (Figure 1.1). In radiation poisoning they are produced from water, and in oxygen poisoning from 
oxygen. As stated by Irwin Fridovich, a Professor of Biochemistry at Duke University: "This was a remarkably prescient theory, considering the paucity of information concerning the generation and scavenging of specific free radicals available at that time" (Fridovich, 1975). In 1969, Joe McCord and Irwin Fridovich went on to solidify the concept of free radical mediation of oxygen toxicity by identifying a catalytic function for the enzyme superoxide dismutase (SOD) (Fredovich and McCord, 1969) providing direct analytical evidence of the in vivo generation of the superoxide radical. Thus, aerobic life is a high wire act supported by $\mathrm{O}_{2}$ chemistry and balanced precariously between the biosynthetic powers provided by the rich energy supplies of respiration and the destructive effects of $\mathrm{O}_{2}$ radicals formed during $\mathrm{O}_{2}$ metabolism.

Microorganisms have a limited tolerance for oxygen. This property is most evident in the cases of obligate anaerobes and microaerophiles, which cannot grow in air saturated media, but it applies as well to committed aerobes, which grow poorly, or even die, when they are exposed to hyperoxia. Yet the basis of this universal phenomenon is not intuitive; after all, the structural molecules from which cells are made, amino acids, lipids, and nucleic acids, are reasonably stable in aerobic buffers (Imlay, 2003).

The chemistry of oxygen plays a major role in its unique behavior. It explains not only why oxygen free radicals are formed, but also why we do not spontaneously combust (despite a favorable energetics).

\subsection{The oxygen molecule}

Oxygen is a paradox. The secret of the behavior of molecular oxygen and its partially reduced species lies in their reduction potentials and molecular orbital structures. 


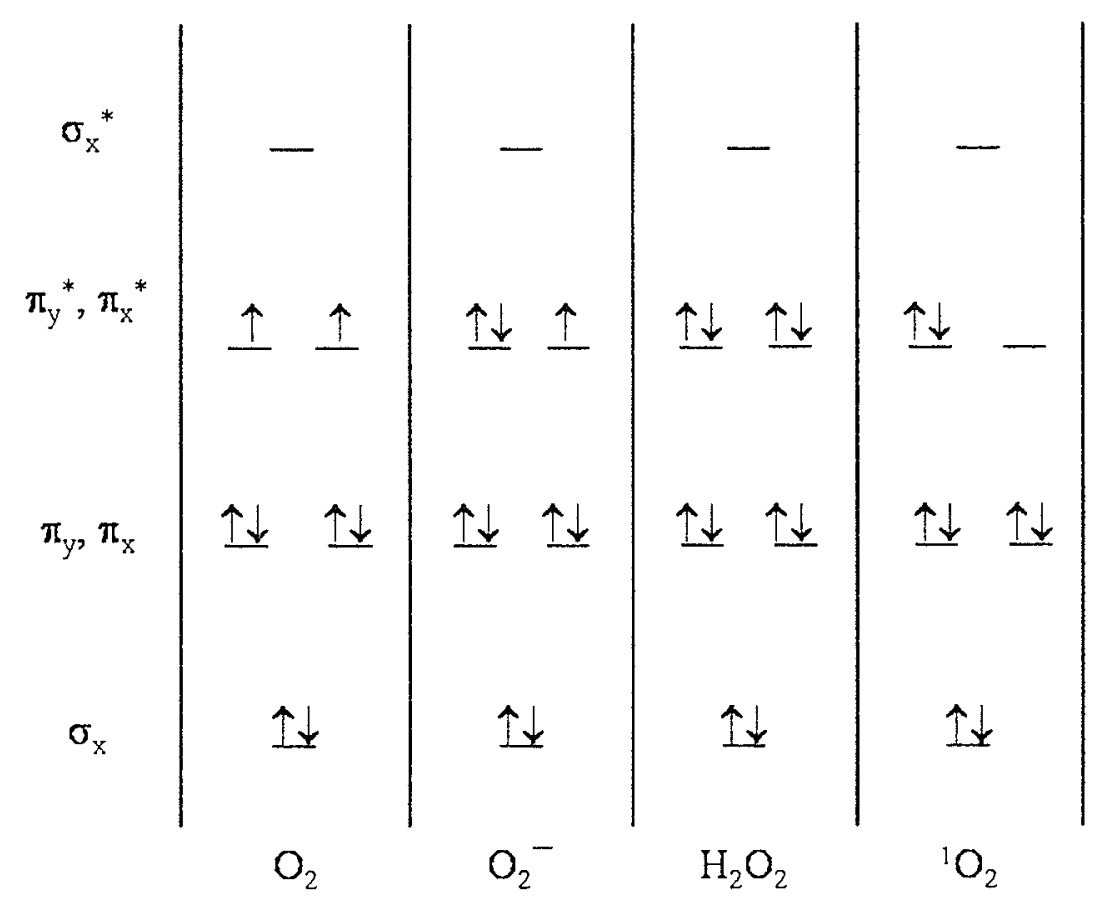

Figure1.2. Molecular orbital diagrams of molecular oxygen $\left(\mathrm{O}_{2}\right)$, superoxide $\left(\mathrm{O}_{2}{ }^{-}\right)$, hydrogen peroxide $\left(\mathrm{H}_{2} \mathrm{O}_{2}\right)$, and singlet oxygen $\left({ }^{l} \mathrm{O}_{2}\right)$. 
Molecular oxygen itself is a rarity, a stable diradical, with two spin-aligned, unpaired electrons in its $\pi$ antibonding orbital (Figure 1.2).

An important consequence of this structure is that organic molecules with spin paired electrons cannot transfer more than one electron at a time to oxygen. Because oxygen is a weak electron acceptor (and most organic molecules are poor electron donors), this restriction ensures that oxygen cannot efficiently oxidize amino acids and nucleic acids. However, the unpaired electrons of dioxygen readily interact with the unpaired electrons of transition metals and organic radicals.

In contrast, the reduction potentials of $\mathrm{O}_{2}{ }^{--}, \mathrm{H}_{2} \mathrm{O}_{2}$ and the hydroxyl radical dictate that in thermodynamic terms they are much stronger univalent oxidants than dioxygen. However, the anionic charge of $\mathrm{O}_{2}{ }^{\circ-}$ inhibits its effectiveness as an oxidant of electron rich molecules, while the reactivity of $\mathrm{H}_{2} \mathrm{O}_{2}$ is diminished by the stability of its oxygenoxygen bond. Neither of these features applies to the hydroxyl radical, and indeed $\mathrm{HO}^{\circ}$ reacts at virtually diffusion rates with most biomolecules.

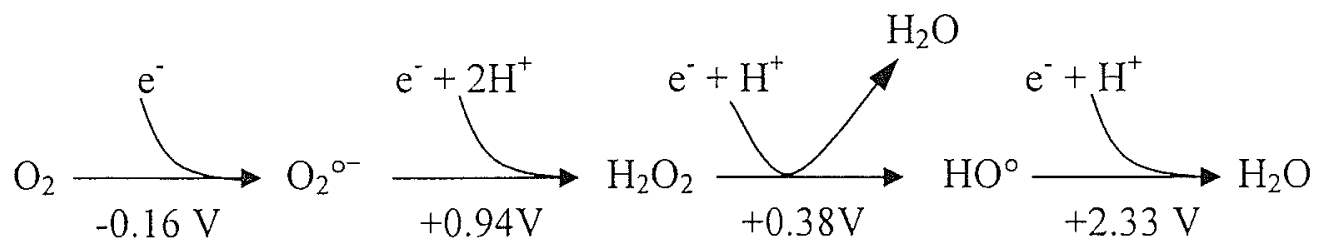

\subsection{Antioxidant Systems}

During the course of evolution, while life adapted to an aerobic atmosphere, all living organisms, both aerobes and anaerobes, evolved mechanisms to protect themselves specifically from oxidative stress and its toxic effects (Sies, 1993; Gutteridge et al., 
1999). During the last 30 years, biochemists and biologists working in this field have thus focused their attention mainly on the following three objects. The first is the identification of the toxic species, the biological targets and the molecular mechanism by which an oxidative stress is expressed within an organism. The second is the role played by oxidative stress in ageing and a number of diseases (neurological disorders, some types of cancers and inflammations, etc.) with the object of developing suitable therapeutic strategies. The third is an understanding of the nature and mechanism of the complex antioxidant machinery which control the balance between the generation and the scavenging of reactive oxygen species, continuously (aerobes) or transiently (anaerobes). These antioxidants include both non-enzymatic and enzymatic species. Historically, the term antioxidants referred to any substance that hindered the reaction of a substance with oxygen. Since every such reaction usually involves radicals, the term "antioxidants" has taken a more mechanistic flavor and now generally refers to any substance which inhibits a free radical reaction (Feldman et al., 1997).

\subsubsection{Non-Enzymatic Defenses}

The non-enzymatic antioxidants include various low molecular weight scavengers such as L-ascorbic acid (Vitamin C), $\beta$-carotene, $\alpha$-tocopherol (Vitamin E), glutathione, phenolic compounds and ubiquinones (coenzyme Q). There are also certain reductants and some iron binding proteins. 


\subsubsection{Enzymatic Defenses}

Enzymatic antioxidants, comprising the primary tier of defense include superoxide dismutases (SODs), catalases, peroxidases, catalase-peroxidases (KatG), and in mammalian cells, glutathione peroxidases (GPx). In addition there is a second tier of defense that includes methionine sulfoxide reductase, proteases, endonucleases and phospholipases (Fridovich, 1999). The major advantage of having specific antioxidant enzymes is that the steady-state concentrations of peroxide or superoxide can be adapted to cellular requirements. Several of these enzymes can be induced, activated or inhibited. The best example of a well studied protein induced in E.coli when grown at high levels of oxygen or exposed to redox-cycling agents is superoxide dismutase (Fridovich, 1995). These agents also induce glucose-6-phosphate dehydrogenase to replenish the NADPH consumed in the antioxidant reactions (such as GSH reductase) (Kao and Hassan, 1985).

GSH levels have been known to increase upon exposure to $\mathrm{H}_{2} \mathrm{O}_{2}$ in $E$. coli and $S$. typhimurum (Christman et al., 1985).

SODs and catalases form a defensive team that prevents the less reactive species $\mathrm{O}_{2}{ }^{\circ-}$ and $\mathrm{H}_{2} \mathrm{O}_{2}$ from being converted into the highly reactive hydroxyl radical $\left(\mathrm{OH}^{\circ}\right)$ (Liochev \& Fridovich, 1992). Superoxide dismutase, first discovered in 1969 (McCord et al., 1969) catalyzes the dismutation of $\mathrm{O}_{2}{ }^{\circ-}$ to $\mathrm{H}_{2} \mathrm{O}_{2}$ and $\mathrm{O}_{2}$ at diffusion limiting rates:

$$
\mathrm{O}_{2}{ }^{--}+\mathrm{O}_{2}{ }^{\mathrm{O}^{-}}+2 \mathrm{H}^{+} \longrightarrow \mathrm{H}_{2} \mathrm{O}_{2}+\mathrm{O}_{2}
$$

These supremely efficient catalysts are abundant in aerobic cells and keep the steady state level of $\mathrm{O}_{2}{ }^{\circ-}$ in the $10^{-10} \mathrm{~mol} \mathrm{l}^{-1}$ range (Imlay and Fridovich, 1991). In E. coli, SODs provide approximately $95 \%$ protection to all targets susceptible to $\mathrm{O}_{2}{ }^{\mathrm{o}-}$ attack (Linochev and Fridovich, 1997). 
There are three classes of SODs that differ in utilizing $\mathrm{Cu}$ and $\mathrm{Zn}, \mathrm{Mn}$, or Fe and Ni. SODs can be cytosolic, localized to specific sub-cellular organelles or secreted (Fridovich, 1997). The presence of SOD enzymes in strictly anaerobic bacteria further supported the notion that $\mathrm{O}_{2}{ }^{\circ}$ is a key and general component of oxidative stress (Hatchikian et al., 1977; Dos Santos et al., 2000). These organisms may be transiently exposed to oxygen and the availability of the antioxidant mechanism may be an advantage (Cypionka, 2000). Some microaerophilic and anaerobic bacteria use not only SOD but also a relatively new class of superoxide-scavenging enzymes, the superoxide reductase (SOR) (Jenney et al., 1999; Lombard et al., 2000; Lombard et al., 2000b., Jovanovic et al., 2000), that catalyze the reduction of superoxide radical anion to $\mathrm{H}_{2} \mathrm{O}_{2}$ :

$$
\mathrm{O}_{2}{ }^{\mathrm{o}}+\mathrm{e}^{-}+2 \mathrm{H}^{+} \longrightarrow \mathrm{H}_{2} \mathrm{O}_{2}
$$

SOD dismutates toxic superoxide anion radical but, in so doing produces hydrogen peroxide, another toxic product, which is degraded by catalases and peroxidases, the latter using organic electron donors (Fridovich, 1978):

$$
\begin{array}{llll}
\mathrm{H}_{2} \mathrm{O}_{2}+\mathrm{H}_{2} \mathrm{O}_{2} & \longrightarrow & 2 \mathrm{H}_{2} \mathrm{O}+\mathrm{O}_{2} & \text { (Catalases) } \\
\mathrm{H}_{2} \mathrm{O}_{2}+2 \mathrm{RH} & \longrightarrow & 2 \mathrm{H}_{2} \mathrm{O}+2 \mathrm{R}^{\circ} & \text { (Peroxidases) }
\end{array}
$$

Catalases, or more correctly hydroperoxidases, are one of the most studied enzymes. The name catalase was first used in 1900 (Loew O, 1900) and the protein has been the object of study in many organisms ever since. Three classes of catalase, unrelated on the basis of sequence and structure exhibit significant catalase activity. The class that is most widespread in nature and which has been most extensively characterized is composed of mono-functional, heme-containing enzymes subdivided based on having large $(>75 \mathrm{kDa})$ or small $(<60 \mathrm{kDa})$ subunits. Phylogenetic analysis has 
demonstrated the existence of two clades of small subunit enzymes and one clade of large subunit enzymes among monofunctional catalases (Klotz et al., 1997). The second less widespread class of catalase is composed of bifunctional, heme containing catalaseperoxidases that are closely related in sequence and structure to plant peroxidases. The third class includes the nonheme or Mn-containing catalases. In addition, a diverse group of proteins, all heme-containing such as chloroperoxidases, plant peroxidases and myoglobins, exhibit very low levels of catalase activity, attributable to the heme, which alone exhibits catalase activity ( Nicholls et al., 2001).

Most bacteria produce one or more catalases that usually respond to oxidative stress, either directly to $\mathrm{H}_{2} \mathrm{O}_{2}$ levels or to the presence of other active oxygen species. In E. coli, the catalase-peroxidase HPI (Hydroperoxidase I), or EcKatG, is controlled as part of the OxyR regulon, which senses active oxygen species, and the monofunctional catalase HPII is controlled as part of the $\sigma^{\mathrm{s}}$ regulon in the stationary phase. Recently, polyamines, including putrescine, spermidine and spermine, were reported to upregulate both the oxyR and rpoS regulons in E.coli (Jung et al., 2003), but the mechanism remains unclear. There is a commonality of a reactive oxygen sensor or growth phase or $\sigma$ transcription factor control mechanism in controlling catalase gene expression, although there is no consistency in the pairing of the type of catalase and the type of regulator.

Like the catalases, peroxidases are predominantly heme-containing enzymes which catalyze the dismutation of hydrogen peroxide using an electron donor other than $\mathrm{H}_{2} \mathrm{O}_{2}(\mathrm{AH})$ :

$$
\mathrm{H}_{2} \mathrm{O}_{2}+2 \mathrm{AH} \text { (reduced) } \longrightarrow 2 \mathrm{H}_{2} \mathrm{O}+2 \mathrm{~A} \text { (oxidized) }
$$


There is a significant variability in peroxidases compared to catalases in regard to their structural properties. This is probably a consequence of the many different roles that individual peroxidases play. While the majority of known peroxidases are hemoproteins, such as horseradish peroxidase (HRP), cytochrome $c$ peroxidase (CCP), and other mammalian, plant and fungal peroxidases, there are also non-heme peroxidases, such as the vanadium containing chloroperoxidase enzyme from thr fungus Cuvularia inaequalis (Messerschmidt and Wever, 1996), the FAD cysteine redox center NADH peroxidase from Streptococcus faecalis (Stehle et al., 1991), and the human peroxiredoxin (peroxidase) (Choi et al., 1998). Peroxidases are discussed in detail in the later sections.

Glutathione peroxidases (GPx) or ascorbate peroxidases are reductases which receive reducing equivalents or are regenerated by electrons from NADPH in metabolic pathways. GPxs are absent in E.coli, but in animal cells these are selenoenzymes, which catalyze the reduction of hydroperoxidases at the expense of GSH (Flohe, 1988; Ursini et al., 1995). The GPx active site contains selenium in the form of the selenocysteine residue (Flohe et al., 1973; Stadtman, 1991). There has been no report of a selenium dependent glutathione peroxidase activity in plants and $\mathrm{H}_{2} \mathrm{O}_{2}$ is degraded by ascorbate peroxidase (Bunkelmann and Trelease, 1996; Smirnoff, 1996).

\subsection{The monofuctional, heme containing catalases}

\subsubsection{Introduction}

All heme-containing catalases have a common two stage mechanism for the degradation of $\mathrm{H}_{2} \mathrm{O}_{2}$. In the first step, one hydrogen peroxide molecule oxidizes the heme 
to an oxyferryl species, the so called compound I, in which one oxidation equivalent is removed from the iron and one from the porpyrin ring to generate a porphyrin cation radical [reaction 1]:

$$
\text { Enz }\left(\text { Por }-\mathrm{Fe}^{\mathrm{Ill}}\right)+\mathrm{H}_{2} \mathrm{O}_{2} \longrightarrow \mathrm{CpdI}\left(\mathrm{Por}^{{ }^{+}}-\mathrm{Fe}^{\mathrm{IV}}=\mathrm{O}\right)+\mathrm{H}_{2} \mathrm{O}
$$

Compound I is a short lived catalytic intermediate with a distinct absorption spectrum characterized by a reduction of absorption intensity of the Sorêt band (at approximately $406 \mathrm{~nm}$; a band diagnostic of hemoprotein). Compound I formation is believed to be initiated by a histidine side-chain in the active site above the plane of the heme. The distal histidine act as a general acid-base catalyst forming a hydrogen bond with the hydrogen peroxide also coordinated to the heme iron. This facilitates the formation of the transition state, which is stabilized by an additional hydrogen bond with an asparagine, leading to scission of the peroxide O-O bond with one oxygen transferred to the iron forming compound I and the second oxygen being connected to water (Fita and Rossman, 1985). A second molecule of hydrogen peroxide is utilized as a reductant of compound I to regenerate the resting enzyme, water and oxygen [reaction 2]:

$$
\mathrm{CpdI}\left(\mathrm{Por}^{\circ}-\mathrm{Fe}^{\mathrm{IV}}=\mathrm{O}\right)+\mathrm{H}_{2} \mathrm{O}_{2} \longrightarrow \mathrm{Enz}\left(\text { Por-Fe }{ }^{\mathrm{III}}\right)+\mathrm{H}_{2} \mathrm{O}+\mathrm{O}_{2}
$$

Despite this common reaction, there are significant differences in reactive capability among the members of this very large family of enzymes (Switala and Loewen, 2002).

Approximately 256 sequences of mono-functional catalase have been divided into three clades arising from a minimum of two gene duplication events (Klotz and Loewen, 2003). Recent induction of catalase-peroxidase and non-heme catalases into the universal tree of life created a larger picture. Clade 1 catalases are predominantly of plant origin, but with one algal representative and a sub group of bacterial origin. Clade 2 enzymes are 
all large subunit enzymes of bacterial and fungal origin. The one archaebacterial clade 2 enzyme is postulated to have arisen in a horizontal transfer event from a bacillus species. The clad 3 enzymes are all small subunit enzymes from bacteria, archaebacteria, fungi and other eukaryotes (Koltz and Loewen, 2003).

Catalases do not follow Michaelis-Menton kinetics except at low substrate concentration; rather, they show pseudo first order saturation kinetics. A comparative study of 16 catalases has shown the great divergence in properties within the catalase family (Switala and Loewen, 2002). Different enzymes are affected differently at higher substrate concentration. Most small subunit enzymes suffer inactivation by $\mathrm{H}_{2} \mathrm{O}_{2}$ at concentrations above $300-500 \mathrm{mM}$ and never reach the Michaelis-Menten $V_{\max }$ predicted by extrapolation from rates at lower substrate concentrations. Large subunit enzymes show inhibition to $\mathrm{H}_{2} \mathrm{O}_{2}$ only at concentrations above $3 \mathrm{M}$, if at all, and exceed the predicted Michaelis-Menten $V_{\max }$. Consequently the presentation of observed data in terms of the typical constants $K_{\mathrm{m}}$ and $V_{\max }$ is misleading because true Michaelis-Menten kinetics are not applicable. Sequence differences among catalases are considered to be responsible for the widely differing reactions rates and substrate affinities, but a rationale for how has not been proposed (Chelikani et al., 2004).

\subsubsection{Structural properties of catalases}

All monofunctional catalases so far characterized are tetrameric, but there are reports of hexamers and an unusual heterotrimer from Pseudomonas aeruginosa which have not yet been substantiated by structural analysis. To date the crystal structures of eleven monofunctional catalases have been reported. These include catalases from: 
bovine liver (BLC) (Murthy el al., 1981, Melik-Adamyan et al., 1986), Penicillium vitale (PVC) (Vanishtein et al., 1981 and 1986), Micrococcus lysodeikticus (MLC) (Murshudov et al., 1982), Proteus mirabilis (PMC) (Gouet et al., 1995), Escherichia coli (HPII) (Bravo et al., 1995 and 1999), human erythrocytes (HEC) (Ko et al., 1999; Puntam et al., 1999), Pseudomonas syrengae (CatF) (Carpena et al., 2001 and 2003), Helicobactor pylori (HPC) (Loewen et al., 2004), Neurospora crasa (Cat 1) (Diaz et al., 2004) and Enterococcus faecalis (Håkansson et al., 2004). The characteristic feature of a catalase structure is a core beta barrel inside which is the deeply buried heme active site accessed by two or three channels (Loewen et al., 2004). In BLC, CATA, HEC, PMC and MLC, there is a common orientation of the active site His imidazole ring over ring III of the heme (His-III orientation) which was concluded to be the normal heme orientation in catalase. In PVC and HPII, the heme orientation is flipped with the active site His located above ring IV of the heme (His-IV) (Murshodov et al., 1996; Chelikani et al., 2004). This flipped orientation in large subunit enzymes is accompanied by oxidation of the heme to heme $\mathrm{d}$ to form a cis-hydroxyspirolactone group on ring III (Murshudov et al., 1996) and suggested that large subunit enzymes are unique. Demonstration of the His-IV orientation in CatF, a clade I catalase, suggested a more general distribution. Recent structurefunction studies on variants of HPII (Chelikani et al., 2003) and CatF (Carpena et al., 2003) have revealed significant information about the channel architecture in catalases.

The deeply buried heme is connected to the surface by three channels. The main channel gives the most obvious access route to the heme as it approaches the heme perpendicular to its plane and has long been considered the primary access route for substrate $\mathrm{H}_{2} \mathrm{O}_{2}$ (Mate et al., 1999; Sevenic et al., 1999; Melic-Adamayan et al., 2001; Chelikani et al., 
2003). The second channel approaches the heme laterally, almost in the plane of the heme, and has been referred as the minor or lateral channel. The third channel has been proposed to connect the heme to the central cavity but the role of this channel has not been proposed (Chelikani et al., 2003).

\subsubsection{An electrical potential field in the channel of mono-functional catalases}

In 2003, Chelikani et al, demonstrated the striking influence of the negatively charged side chain of a conserved Asp (181 in HPII) on catalase activity. When replaced with any uncharged residue, polar or non-polar, including Asn, Ala, Ser, and Ile, enzymatic activity was lost accompanied by a reduction in solvent occupancy in the channel including the sixth ligand water in all subunits of Asp181Ala, Asp181Ser, and Asp 181 GIn. However, variant Asp181Glu exhibited normal levels of activity and contained the sixth ligand water in all subunits as part of an unbroken water matrix extending the full length of the channel. The existence of an electrical potential field in the hydrophobic portion of the channel between the negatively charged carboxylate and positively charged heme iron was proposed as an explanation for the phenomenon. The potential field influences the orientation of any molecule with an electrical dipole including both $\mathrm{H}_{2} \mathrm{O}$ and $\mathrm{H}_{2} \mathrm{O}_{2}$. In both cases, the preferred orientation will be with oxygen atoms pointed toward the positively charged heme iron and the hydrogens toward the negatively charged side chain of Asp or Glu. The uniform orientation of waters favours hydrogen bond formation and explains the high water occupancy with both Asp181 and the variant Asp181Gln. A second result of the induced orientation of hydrogen peroxide is that it will enter the active site with the a-oxygen oriented toward 
the heme iron and the a-hydrogen on this oxygen located within the hydrogen bonding distance of the $\mathrm{NH}_{2}$ of the active-site Asn, thereby explaining conclusion from molecular dynamic studies that the substrate hydrogen peroxide enters the active site in a preferred orientation (Kalko et al., 2001).

\subsection{Non-heme or manganese containing catalases}

There is another group of catalases that, instead of heme, contains a manganese complex at the reaction center. This group was initially referred to as pseudo-catalases because the enzyme lacked a heme prosthetic group (Kono and Fridovich, 1983), but now are more commonly called Mn-containing catalases (Allgood and Perry, 1986), with the terms non-heme (Nicholls, 2001) and dimanganase catalase (Antonyuk et al., 2000) also being used. These are not as widespread as the heme-containing catalases, being found only in eubacteria with the exception of Pyrobaculum calidifontis (a facultative aerobic respiratory, hypertherophylic Chrenarcheabacterium). They show lower enzymatic activity than heme-containing catalases with the exception of $P$. calidifontis catalase (Koltz et al., 1997).

\subsection{Monofunctional peroxidases}

Peroxidases are predominantly heme containing and catalyze the hydrogen peroxide mediated oxidation of various organic and inorganic substrates generating in some cases, free radial species (Hillar et al., 1999). The overall reaction can be summarized as:

$$
\mathrm{H}_{2} \mathrm{O}_{2}+2 \mathrm{AH} \text { (reduced) } \longrightarrow 2 \mathrm{H}_{2} \mathrm{O}+2 \mathrm{~A} \text { (oxidized) }
$$


Typical reducing substrates include aromatic phenols, phenolic acids, indoles, amines and sulfonates although inorganic ions are used by a few enzymes (Preston et al., 1987). The reaction proceeds in three steps, with the first step involving formation of Compound I, a high oxidation state intermediate comprising an Fe(IV) oxoferryl centre and a porphyrinbased cation radical [reaction 2], common to both catalases and pereoxidases:

$$
\begin{aligned}
& \mathrm{Enz}\left(\text { Por- }-\mathrm{Fe}^{\mathrm{III}}\right)+\mathrm{H}_{2} \mathrm{O}_{2} \longrightarrow \mathrm{Cpdl}\left(\mathrm{Por}^{{ }^{+}}-\mathrm{Fe}^{\mathrm{lV}}=\mathrm{O}\right)+\mathrm{H}_{2} \mathrm{O} \\
& \mathrm{CpdI}\left(\mathrm{Por}^{\circ}{ }^{+}-\mathrm{Fe}^{\mathrm{IV}}=\mathrm{O}\right)+\mathrm{AH}_{\text {red }} \longrightarrow \mathrm{CpdII}\left(\mathrm{Por}-\mathrm{Fe}^{\mathrm{IV}}-\mathrm{OH}\right)+\mathrm{A}_{0 \mathrm{x}} \\
& \text { CpdII (Por-Fe } \left.{ }^{I V}-\mathrm{OH}\right)+\mathrm{AH}_{\text {red }} \longrightarrow \text { Enz }\left(\text { Por-Fe }{ }^{I I I}\right)+\mathrm{A}_{\mathrm{ox}}
\end{aligned}
$$

A transient intermediate (compound 0 ) formed prior to compound I has been detected in reactions between $\mathrm{HRP} C$ and $\mathrm{H}_{2} \mathrm{O}_{2}$ at low temperatures and described as an $\mathrm{Fe}$ (III)hydroperoxy complex (Filizola and Loew, 2000). In formal terms, compound I is two oxidizing equivalents above the resting state. The first one-electron reduction step requires the participation of a reducing substrate and leads to the generation of compound II, an $\mathrm{Fe}(\mathrm{IV})$ hydroxoferryl species that is one-oxidising equivalent above the resting state [reaction 3]. Both compound I and compound II are powerful oxidants, with redox potentials estimated to be close to $+1 \mathrm{~V}$. The second one-electron reduction step returns compound II to the resting state of the enzyme. Reaction of excess hydrogen peroxide with the resting state enzyme gives compound III, which can also be prepared by several other routes (Dunford, 1991). This intermediate is best described as a resonance hybrid of iron (III)-superoxide and iron (II)-dioxygen complexes. A high-resolution crystal structure of $95 \%$ compound III shows dioxygen bound to heme iron in a bent conformation (Berglund et al., 2002), . A similar but irreversibly inactivated compound 
IV formation has also been reported (Baynton et al., 1994). A covalently modified heme iron, possibly with a perhydroxy or dioxygen group in a compound III like structure, has been reported in the crystal structure of Helicobacter pylori catalase (HPC), with and without formic acid bound, (Loewen et al., 2004). In the HPC structure, the elongated electron density associated with the iron in subunit B resembles the maps of oxymyoglobin (Vojtechovsky et al., 1999) and compound III of horseradish peroxidase (Berglund et al., 2002). High resolution crystal structures of the oxidised intermediates of HRP C has shown that distal heme pocket residues Arg38 and His42 (conserved in all members of the plant peroxidase superfamily) play a significant role in the formation of compound I (Berglund et al., 2002).

In 1992, Welinder performed multiple sequence alignment of number of peroxidases and classified them into three distinct structural families or classes. Class I enzymes are identified as bacterial peroxidases, and include the sequence of $E$. coli catalase-peroxidase and yeast cytochrome $c$ peroxidase. Class II enzymes include the plant peroxidases such as horseradish peroxidase isoenzyme $\mathrm{C}$ while class III contains the classical secretory plant peroxidases for example horseradish peroxidase. It should be noted that the structural classes identified do not imply degrees of evolutionary relatedness, simply structural variation (Hillar, 2000). Among class I enzymes, for example, the yeast cytochrome c peroxidase has been shown to be evolutionarily distinct from bacterial peroxidases (KatGs) by phylogenetic analysis (Klotz, 1997). 


\subsection{Catalase-peroxidases}

Catalase-peroxidases, also called KatGs after the encoding gene $k a t G$ (Loewen et $a l, 1985$ ), are found in bacteria, archaebacteria and a few fungi. In 1992, catalaseperoxidases gained significant notoriety with the reports confirming that mutations in the $k a t G$ gene encoding the Mycobacterium tuberculosis catalase-peroxidase (MtKatG) were responsible for resistance against isoniazid (Zhang et al., 1992), the front line antituberculosis drug, and were the leading cause of the world-wide resurgence of tuberculosis (Deretic et al., 1996). This gave rise to significant interest among researchers, worldwide. These fascinatingly complex enzymes show strong catalatic activity, although with turnover rates only 1-20\% of mono-functional catalases and a broad spectrum peroxidatic activity. They have recently been associated with a slow NADH oxidase, hydrazinolysis and isonicotinyl NAD synthase activities (Singh et al., 2004). The catalase-peroxidase HPI of E. coli was first purified and characterized in 1979 (Claiborn and Fridovich, 1979), and its encoding gene $k a t G$, was sequenced in 1988

(Triggs-Raine et al., 1988), providing the first catalase-peroxidase sequence and demonstrating the sequence similarity to plant peroxidases. Since then a wide variety of KatGs have been purified, characterized and sequenced.

\subsubsection{Phylogeney of catalase-peroxidases}

Catalase-peroxidases bear significant sequence similarity to eukaryotic class I heme peroxidases of the plant, fungal and bacterial peroxidase superfamily (Welinder $e t$ al., 1992). This superfamily includes three independent evolutionary lineages depending upon the cellular location and function of the enzyme. Class I (peroxidases of prokaryotic 
origin) contains the large family of ascorbate peroxidases from higher plants and green algae, cytochrome $c$ peroxidase $(\mathrm{CcP})$ from Saccharomayces cerevisiae, and catalaseperoxidases. Class II comprises extracellular fungal peroxidases (e.g lignin peroxidase, manganese peroxidase), while class III contains the classical secretory plant peroxidases (e.g horseradish peroxidase) (Zàmocký et al., 2000). All representatives possess the same heme prosthetic group containing high spin ferric heme. Catalase peroxidases are the only member of this superfamily that possesses notable catalase activity (i.e. they can reduce and oxidize hydrogen peroxide). All other members of the superfamily are restricted to reducing hydrogen peroxide with subsequent oxidation of a secondary substrate. These 'noncatalase' members of class I exhibit strong specificity for electron donors: the preferred substrate is ascorbate in the case of ascorbate peroxidase and cytochrome $c$ in case of cytochrome $c$ peroxidase. The in vivo substrate for the peroxidatic reaction of catalase-peroxidases remains unidentified. Sequence analysis has revealed that catalase-peroxidases have evolved from a common ancestor, shared with class I, II and III peroxidases (Zàmocký et al., 2000). The core of the enzyme is a nonbundled $\alpha$-helical fold consisting of ten helices with bound ferric protoporphyrin IX (Zàmocký et al., 2000), but KatGs arose from a gene duplication event, giving rise to a two domain structure in which the $\mathrm{N}$-terminal domain is active and the C-terminal domain is inactive (Welinder, 1992). The first review on KatG phylogeny included 19 sequences (Faguy et al., 2000) while the most recent has 60 sequences (Zàmocký et al., 2004). With the large number of sequences in the data set, the tree is less robust than previous trees that were based on a much smaller set of selected sequences (Klotz et al., 1997; Faguy and Doolittle, 2000; Loewen et al., 2000) leading to several possible 
interpretations of relationships. Upon integration into a conceptual tree of life, it is apparent that KatGs evolved much later than the heme-containing mono-functional catalases, and a significant frequency of lateral gene transfer is evident (Klotz and Loewen, 2003). A recent report has also supported this by analyzing sixty representative sequences covering all known subgroups of class I of the super-family of bacteria, fungal and plant heme peroxidases, showing that the duplication event in $k a t G$ occurred at a later phase of evolution (Zàmocký et al., 2004).

\subsubsection{Regulation of catalase-peroxidase gene $(\mathrm{kat} G)$ expression.}

Regulation of KatG normally occurs through the OxyR regulator belonging to the LysR family. OxyR is an $\mathrm{H}_{2} \mathrm{O}_{2}$ sensing transcriptional regulator and transducer of oxygen stress. It is activated by disulfide bond formation between two cysteine residues and induces the expression of $\operatorname{xxyS}$ (which encodes a small, nontranslated regulatory RNA), $k a t G$ (which encodes hydrogen peroxidase I), $a h p C$ (which encodes alkyl hydroperoxide reductase), gorA (which encodes glutathione reductase), $d p s$ (which encodes DNA binding protein), and grxA (which encodes glutaredoxin 1). Glutaredoxin 1 deactivates OxyR by reducing the disulfide bond, forming an autoregulatory feedback loop (Zheng et al., 1998). Only the oxidized form of the protein is able to activate the expression of downstream genes and negatively autoregulate its expression by binding to specific regions in promoters (Christman et al., 1989). Irrespective of its redox state, OxyR also acts as a repressor of its own expression like other LysR transcriptional regulators. Several oxyR genes have been identified in other organisms, such as Burkholderia pseudomallei (Loprasert et al., 2002), Haemophilus influenzae (Maciver and Hensen, 
1998), various Mycobacterium species (Dertci et al., 1995, Dhandayuthapani et al., 1997), Xanthomonas species (Loprasert et al., 1997), and even the anaerobic bacterium Bacteroides fragilis (Rocha et al., 2000). In E. coli, kat G is transcriptionally induced during the logarithmic phase in response to low concentrations of $\mathrm{H}_{2} \mathrm{O}_{2}$, and requires the transcriptional regulator, OxyR (Visick et al., 1997). Conversely, monofunctional HPII is induced during the transition from the exponential phase to stationary phase by RpoS, an alternative sigma factor, which is neither regulated by Oxy R nor induced by $\mathrm{H}_{2} \mathrm{O}_{2}$ (Loewen et al., 1985; Mukhopadhyay et al., 1994; Mulvey et al., 1990; Schellhorn et al., 1992). M. tuberculosis has a non functional OxyR gene with several mutations making it a natural mutant of OxyR (Deretic et al., 1995; Sherman et al., 1995). In all the mycobacterial species studied, the DNA region immediately upstream of the $k a t G$ genes is highly conserved and encodes the fur $A$ gene, suggesting a putative involvement of the FurA protein in katG regulation (Anna et al., 2001). In M. smegmatis, a knockout fur A mutant over expressed the catalase-peroxidase $\mathrm{KatG}$ and was complemented by expression of FurA suggesting that FurA is a negative regulator of kat $G$. Fur-like proteins are transcriptional repressors that exhibit a $\mathrm{Fe}^{2+}$-dependent DNA binding activity and regulate several genes involved in iron metabolism (Escolar et al., 1999). There is an intimate relationship between iron metabolism and oxidative stress as the cytotoxic effects of reactive oxygen species are largely mediated by iron, and regulators of $E$ coli involved in oxidative stress response, OxyR and SoxRS, activate the expression of Fur (Zheng and Storz, 2000). E. coli $\Delta$ fur mutants are more sensitive to hydrogen peroxide than are Fur-proficient strains (Touati et al., 1995). In M. smegmatis, hydrogen peroxide sensitivity is increased by iron starvation (Lundrigan et al., 1997); in Staphylococcus 
aureus, Fur is necessary for oxidative stress resistance (Horsburgh et al., 2001); and in Streptomyces spp., Fur-like proteins regulate catalase-peroxidase genes in an irondependent manner (Zou et al., 1999).

\subsubsection{Structure and Mechanism of catalase-peroxidases}

Catalase-peroxidases are unique in modulating substantial catalase and peroxidase activity from a single enzyme. Despite the lack of similarity in the sequence and structure between catalase-peroxidases and mono-functional catalases, they share a common catalatic reaction for the compound I formation [reaction 1]. Biochemical and structural characterization of KatGs suggested this common reaction could be attributed to the active sites of both enzymes, which contain heme and have similar roles for the active site residues, though in catalase-peroxidases asparagine is replaced by the arginine or tyrosine (Carpena et al., 2003; Jakopitsch et al., 2003). In catalases, the compound I is reduced to the ground state in a single two-electron reduction step using a second molecule of hydrogen peroxide while the peroxidatic reaction requires an electron doner to reduce the compound I to the ground state in two one-electron reduction steps [reactions 2 and 3]. A variety of organic substances, such as 0 -dianisidine, ABTS and pyragallol, act as the electron donating substrate, in vitro, for the peroxidatic reaction, but in vivo, the peroxidatic substrate remains unknown.

Attempts to crystallize KatGs were unsuccessful until 2001 when the first report describing the crystal structure of the halophilic archaebacterium Haloarcula marismortui (HmKatG; Yamada et.al., 2001, 2001) appeared. This was followed by the 

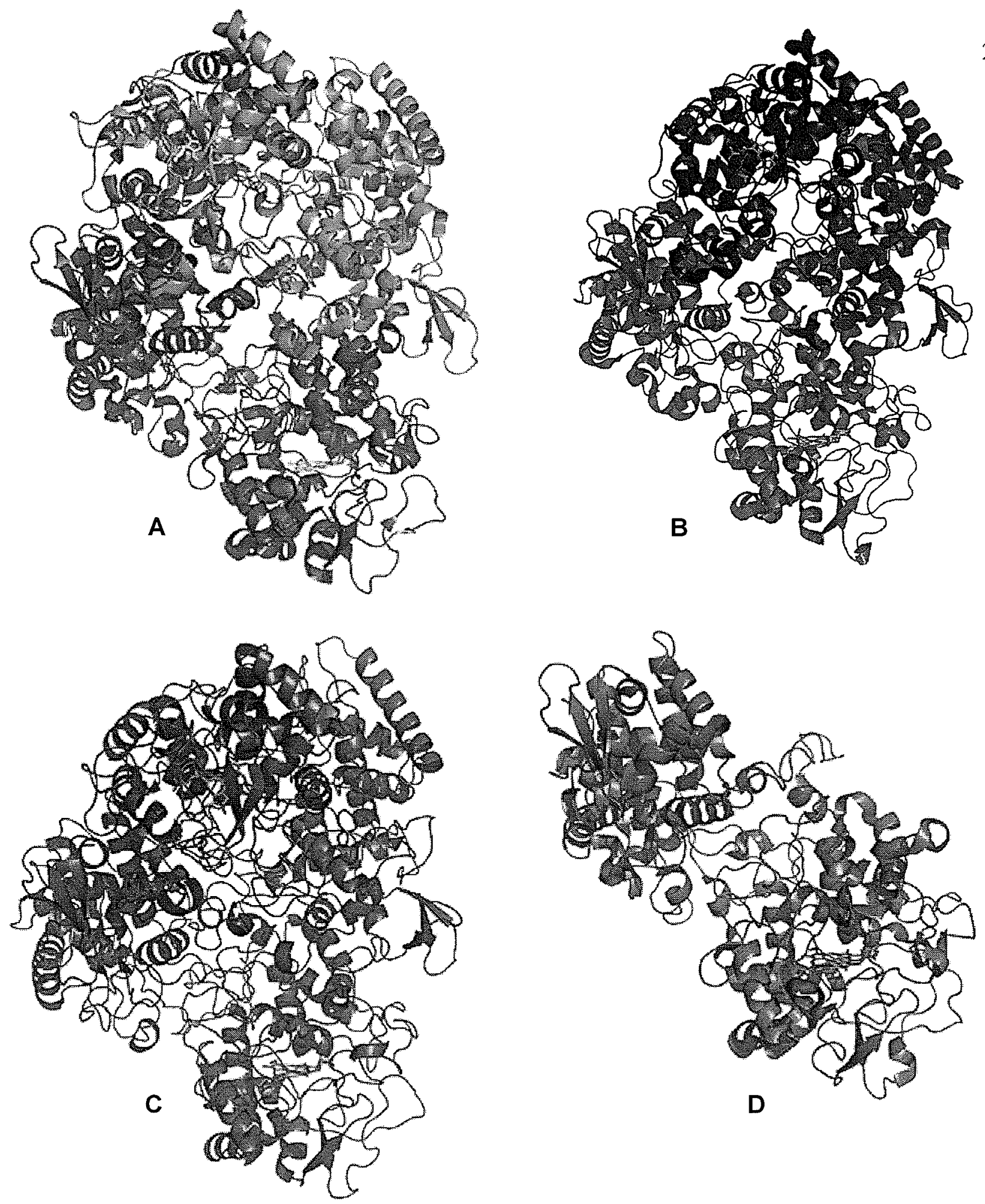

Figure 1.3. Structures of catalase-peroxidase (KatG) from Burkholderia pseudomallei (A), Mycobacterium tuberculosis (B), Haloarcula marismortui (C), and Synechocystis PCC 7942 (D). Each subunit is shown with different colour and the heme is shown in green. Ribbon representations are prepared using Pymol (Deleno, 2002)) and the coordinates submitted to the protein data bank. 


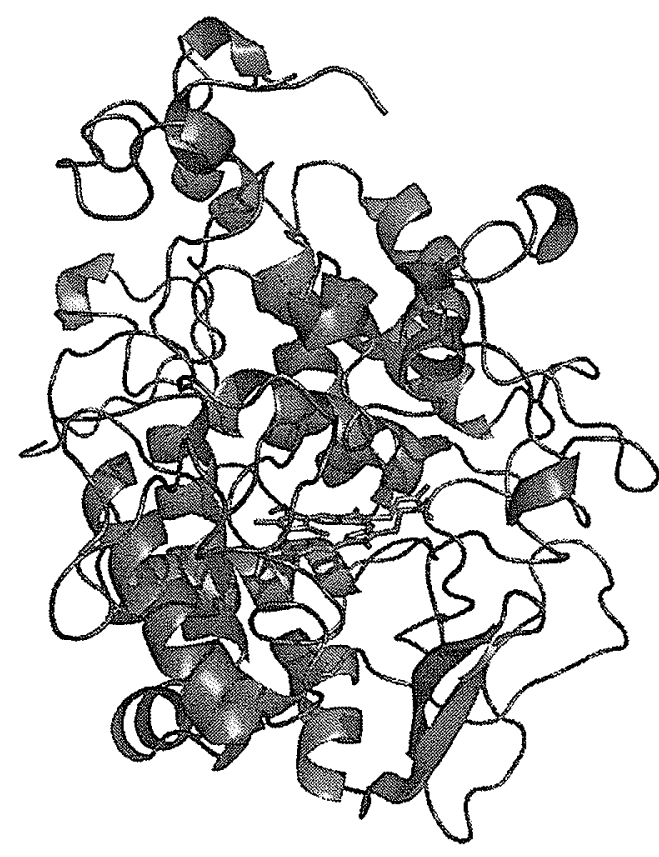

A

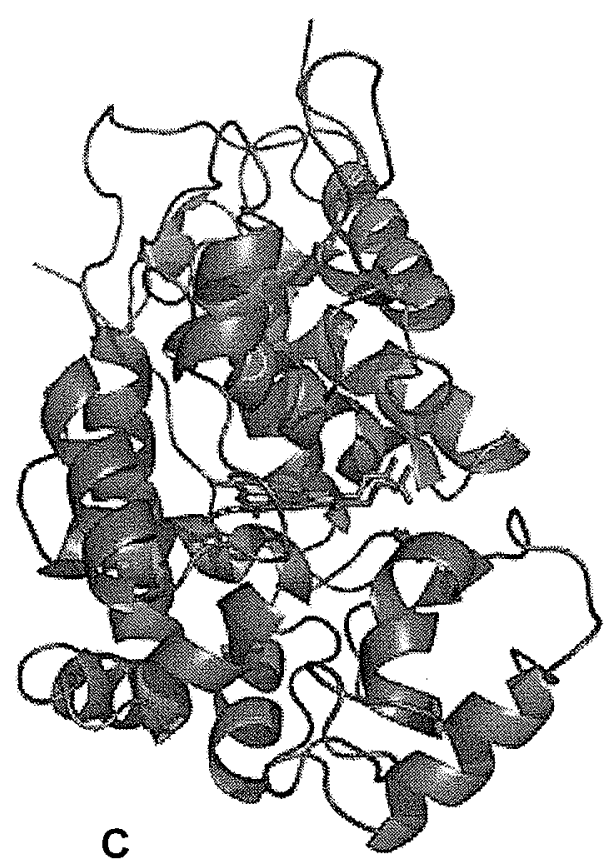

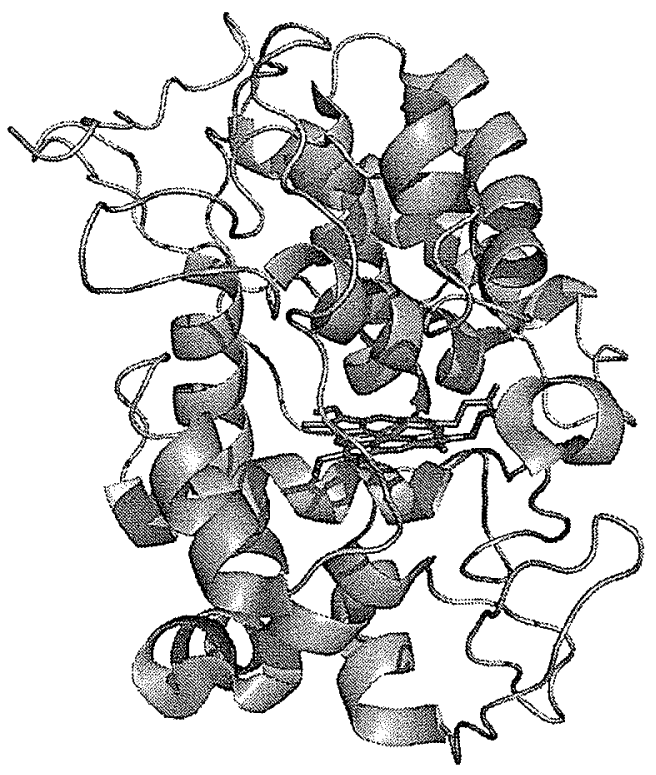

B

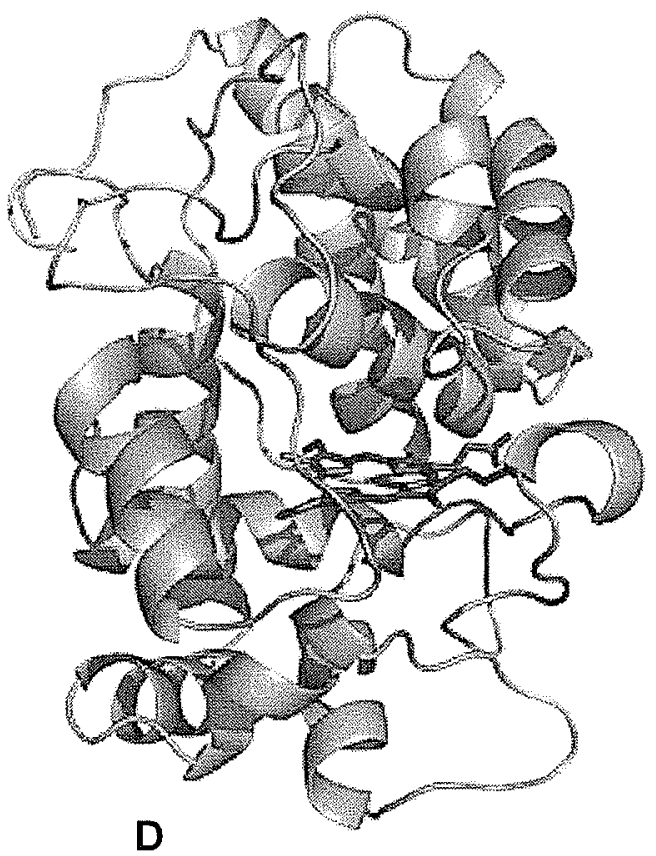

Figure 1.4. Structures of the $\mathrm{N}$-terminal of BpKatG (A), cytochrome $c$ Peroxidase (B), Horseradish peroxidase (C), and ascorbate peroxidase (D). The heme is shown in green.Ribbon representations are prepared using Pymol (Deleno, 2002) and the coordinates submitted to the protein data bank. 
crystal structure reports of KatGs from Burkholderia pseudomallei (BpKatG; Carpena et al., 2002, 2003), Synechococcus (SyKatG; Wada et al., 2002) and Mycobacterium tuberculosis [Figure 1.3] (Bertrand et al., 2004). In addition, the crystal structure of the Cterminal domain of E. coli HPI has been reported (Carpena et al., 2004).

Crystal structure analysis confirms that KatGs are normally active as homodimers, although homotetrameric associations do occur, as in EcKatG or HPI. The individual subunits are relatively large at approximately $80,000 \mathrm{Da}$ and have two distinct but sequence related domains, possibly fused during a gene duplication event (Welinder, 1991). During subsequent evolution, the $\mathrm{N}$-terminal domain retained (or acquired) the ability to bind heme and the C-terminal domain lost (or never acquired) this ability. Despite this functional divergence, considerable structural homology has been conserved, with a root mean square deviation (r.m.s.d) of $2.19 \AA$ for the $\mathrm{C} \alpha$ atoms of 133 residues in the ten $\alpha$ helical sections that are topologically equivalent in the two domains of BpKatG. The two subunits in the molecule are associated mainly through interactions between the $\mathrm{N}$ - and $\mathrm{C}$-terminal domains, with few $\mathrm{N}$-terminal to $\mathrm{N}$-terminal and no $\mathrm{C}$-terminal to $\mathrm{C}$ terminal domain interactions.

A role for the C-terminal domain, other than as an agent for dimer formation and stabilization, has not been determined (Carpena et al., 2004). The heme is located in the $\mathrm{N}$-terminal region (Figure 1.3) and the heme environment in KatG has been shown to be similar to that of plant peroxidases [Figure 1.4] (Yamada et al., 2002; Carpena et al., 2003; Wada et al., 2002; Bertrand et al., 2004). On the distal side of heme, the active site triad of Arg108, Trp111, and His1 12 (BpKatG numbering) is typical of all 
catalase-peroxidases and of class I peroxidases. On the proximal side, His279 is the fifth ligand to the heme iron atom also as in the peroxidases, and it is in close association with the fully conserved Asp389, which interacts with the indole $\mathrm{N}$ atom from the proximal Trp330 (Carpena et al., 2003) as shown in Figure 1.5.

Crystal structures from four KatGs and one variant from BpKatG (Ser324Thr, Deemagaran et al., 2005) and MtKatG (Ser315Thr Zhao et al., 2006) has provided better insight into the structural properties but there is no consensus on the structural basis for the high catalase activity of KatGs. It is not clear whether the role of KatG compound I is the same as in monofunctional catalases or it follows a different path. KatG's thermodynamics present no barrier to the two electron oxidation of hydrogen peroxide to dioxygen. The standard reduction potential of the redox couple $\mathrm{E}^{\circ}\left(\mathrm{O}_{2} / \mathrm{H}_{2} \mathrm{O}_{2}\right)$ is $280 \mathrm{mV}$ (Wood, 1988), and normally the oxidizing intermediates of heme peroxidases are strong oxidants (Dunford, 1999).

It has previously been shown that $\mathrm{KatG}$ compound I, like that of monofunctional catalases, does not accumulate during $\mathrm{H}_{2} \mathrm{O}_{2}$ degradation but can be trapped by using organic peroxides (Jakopitsch et al., 1999). However, this intermediate shows a low reactivity towards $\mathrm{H}_{2} \mathrm{O}_{2}$ (Jakopitsch et al., 1999). Also, both in the absence and in the presence of one electron donors, accumulation of a hydroxo ferryl like compound II was never observed in wild type KatGs, suggesting that the spectral signature of $\mathrm{KatG}$ compound II could be similar to that of the ferric protein (Jakopitsch et al., 1999; Reglesberger et al., 2000). Unlike plant peroxidases, in KatGs the formation of compound III has not be confirmed even at higher concentration of $\mathrm{H}_{2} \mathrm{O}_{2}$ (Chauchane et al., 2000). Besides these mechanistic peculiarities, KatG's structural analysis has 

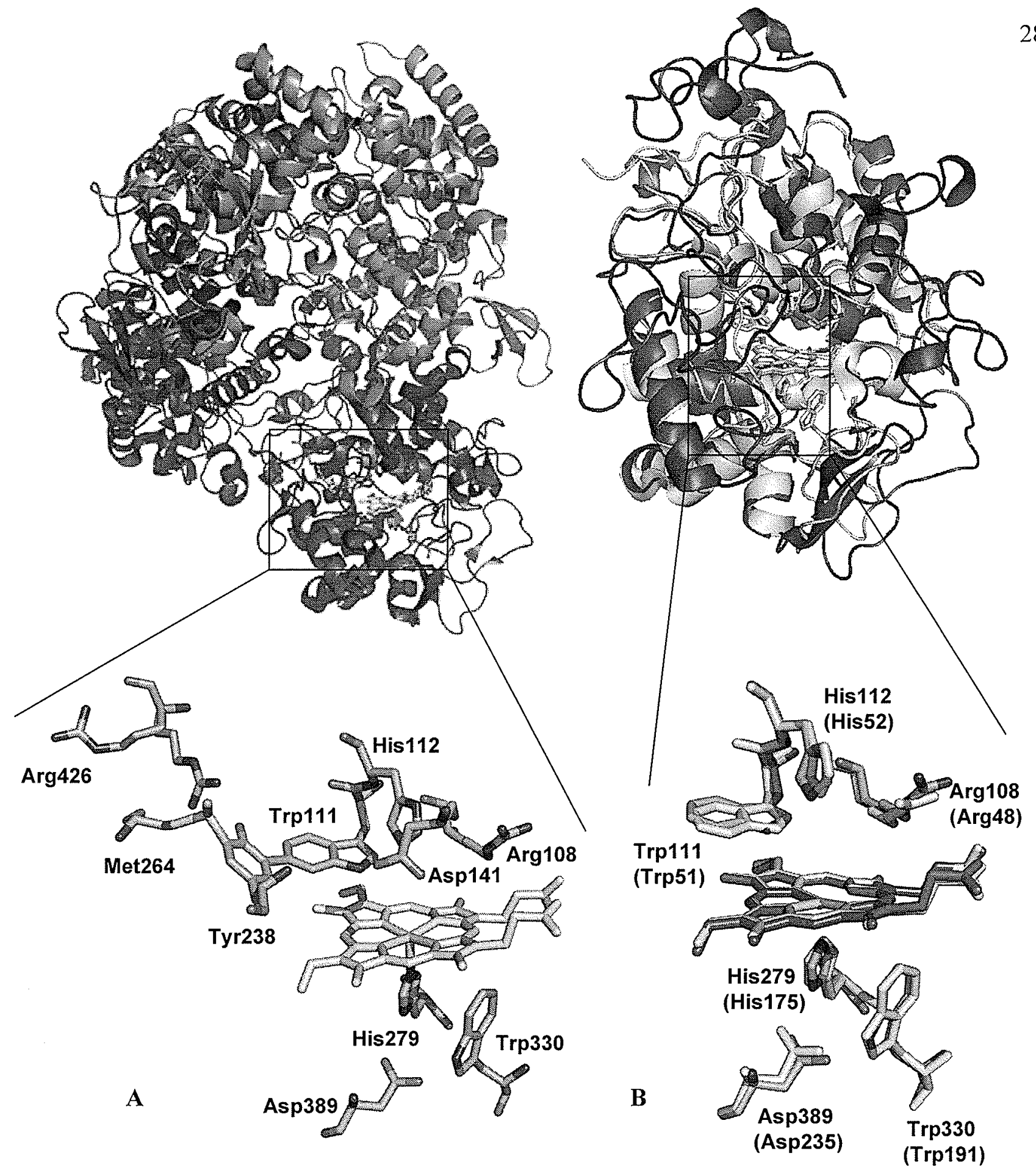

Figure 1.5. Panel $A$ shows the active site residues in the proximal and distal side of BpKatG. In the panel B, an overlay of cytochrome $c$ peroxidase with the $\mathrm{N}$-terminal of BpKatG has been shown. KatG's subunits are shown with different colours and $\mathrm{CcP}$ is shown in yellow and parenthesis indicates residues numbering in CcP. Figures are prepared using Pymol (Deleno, 2002) and the coordinates submitted to the protein data. 
revealed some striking difference from heme peroxidases as discussed in the following sections.

\subsubsection{The unusual covalent modifications}

In a wide range of metalloenzymes, active site residues undergo covalent modification such as in cytochrome $c$ oxidase (His240-Tyr244; heme $\left.{ }_{\mathrm{a} 3}-\mathrm{Cu}_{\mathrm{B}}\right)($ Ostermeier et al., 1997; Yoshikawa et al., 1998; Buse et al., 1999), catalase HPII (His392-Tyr415; heme) (Bravo et al., 1997), galactose oxidase (Tyr272-Cys228; copper) (Ito, et al., 1994; Ito, et al., 1994), catalase 1 (Cys-356-Tyr379; heme) (Diaz et al., 2004), and amine oxidases (2,4,5 trihydroxyphenylalanine quinine; copper) (Parsons et al., 1995). In KatGs three unusal covalent modifications has been reported. The most prominent modification is the cross linking of three amino acid side chains, Trp111, Tyr238 and Met264 (BpKatG numbering) located on the distal side of the heme [Figure 1.6a] and has been reported in the crystal structure reports of all four KatGs and the variant Ser324Thr of BpkatG (Yamada et al., 2002; Carpena et al., 2003; Wada et al., 2002; Bertrand et al., 2004; Deemagaran et al., 2005).

This modification has also been confirmed by mass spectrometric analysis of BpKatG (Donald et al., 2003) and MtKatG (Ghiladi et al., 2005; Ghiladi et al., 2005a). Met-Tyr-Trp crosslink appears to be a characteristic common to all KatGs and has been shown to be essential for catalase activity (Donald et al., 2003; Jakopitsch et al., 2003; Ghiladi et al., 2005; Santoni et al., 2004; Jakopitsch et al., 2003a; Jakopitsch et al., 2004; Ghiladi et al., 2005a). Similar cross-link has not been reported in heme peroxidases and these distal tyrosine and methionine are not conserved. Other modifications include a perhydroxy group added to the vinyl group on the ring I of the heme of BpKatG [Figure 


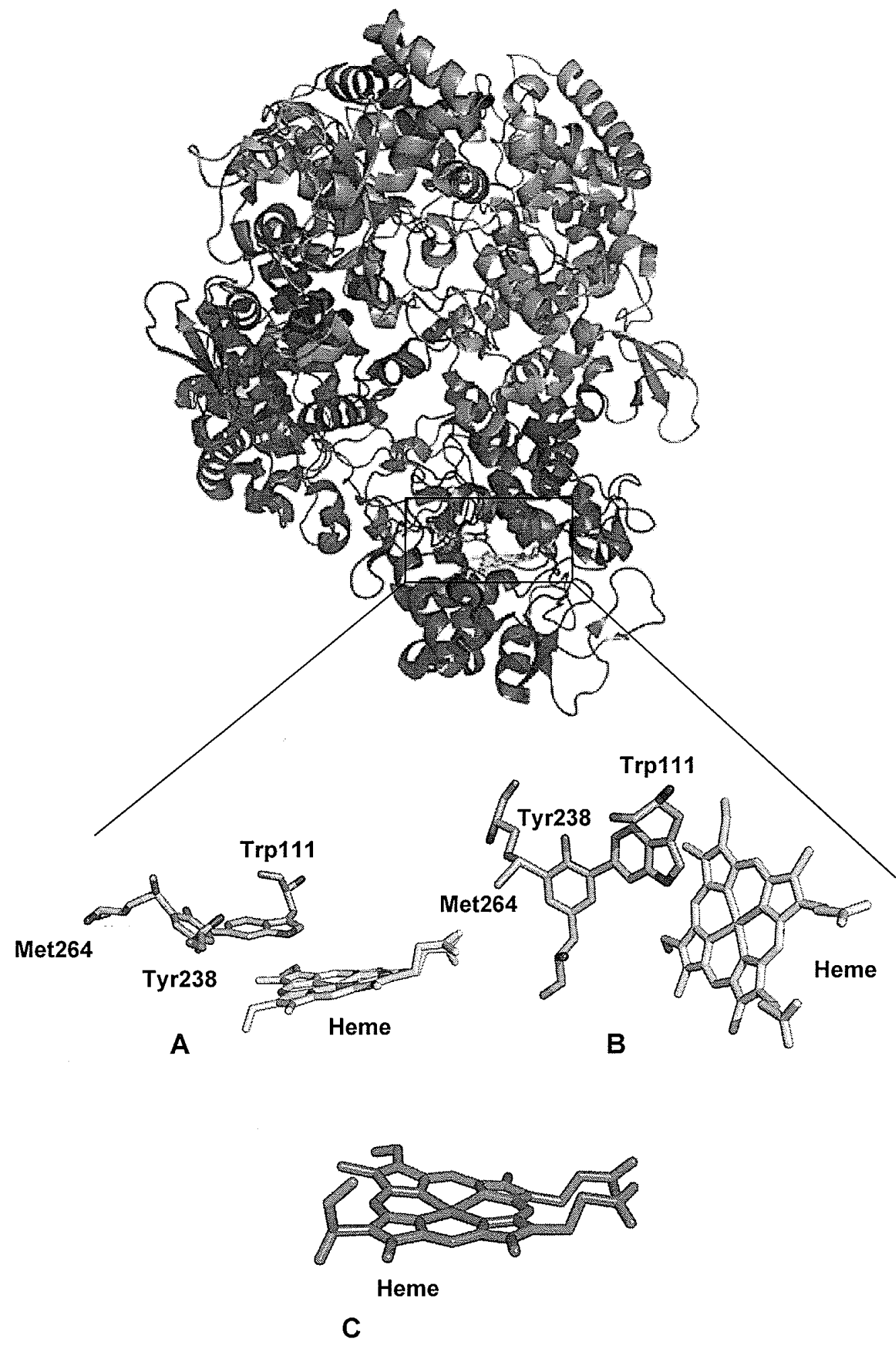

Figure 1.6. Structural representation of covalent modifications in BpKatG: Trp111-Tyr238-Met264 cross link has been shown in two views; A is rotated almost $90^{\circ}$ to B. Panel C shows the peroxy modification (shown in red) on heme. Figures are prepared using Pymol (DElono, 2002) the coordinates submitted to the protein data bank. 
1.6b] (Carpena et al., 2003) and a perhydroxy group on the indole $N$ of $\operatorname{Trp} 111$ of BpKatG (Deemagarn et al., 2005; Carpena et al., 2006).

\subsection{The covalent Trp111-Tyr238-Met264 cross link}

The crossed linked peptide (Trp-Tyr-Met) in KatGs has been subjected to extensive study using structural, mass spectrometric and HPLC analysis. Studies on BpKatG demonstrated that the covalent adduct involves the indole ring of the active site Trp111 and the sulfur of Met264 joined to the two ortho positions of Tyr238, and is found in close proximity to the heme [Figure1.6a] (Donald et al., 2003). Trp111 is a catalytic residue required for compound I reduction in the catalatic reaction (Hillar et al., 2000 and Jakopitsch et al., 2003). Possible roles suggested for the covalent adduct include stabilizing the indole ring in the active site and providing a route for electron transfer. Mass spectrometric analysis of BpKatG, EcKatG (Donald et al., 2003), Synechocystis $\mathrm{KatG}$ (Jakospitch et al., 2003) and MtKatG (Ghiladi et al., 2005; Ghiladi et al., 2005a) have confirmed the presence of the Trp-Tyr-Met adduct in the five catalaseperoxidases and supports the conjecture that the structure may be common to all catalaseperoxidases (Smulevich et al., 2006).

Recently, De Montelleno demonstrated that formation of the Met-Tyr-Trp crosslink is autocatalytic and requires compound I as the two electron oxidizing species rather than compound II (Ghiladi et al., 2005). A comparison of apoMtKatG with holoMtKatG demonstrated the lack of cross link in holoenzyme and its reconstitution with heme alone did not generate the cross link rather a 33\% cross link formation was 
observed after incubating the reconstituted enzyme with PAA (peroxy acetic acid) (thus generating compound I) but generation of compound II by incubating the enzyme with even an higher concentration of MMMP (2-methyl-1-phenyl-2-propyl hydroperoxide), leads to a negligible increase in the cross link present. Auther attributed higher efficiency (as compared to $33 \%$ ) in vivo to the presence of bound substrate.

The cross link formation has been proposed to be a sequential process with both Trp107 and Tyr229 (MtKatG numbering) necessary for complete Met-Tyr-Trp crosslink. Variants of Met255 still produced a Tyr-Trp linkage despite the lack of a Met-Tyr linkage, suggesting that the cross link forms first between the $\mathrm{C \eta} 2$ of $\operatorname{Trp} 107$ and $\mathrm{C} \varepsilon 1$ of Tyr229, followed by bond formation between the CE2 of Tyr229 and S $\delta$ of Met264 (Jakopitsch et al., 2003; Ghiladi et al., 2005). Thus, the over all reaction starts with compound I formation either by a peracid (in vitro) or hydrogen peroxide (in vivo) leading to oxidation of both Tyr229 and Trp 107 which, being in close proximity to the heme periphery ( $\sim 3.7 \AA$ and $5.9 \AA)$, remains within the threshold for long range electron transfer (Gray and Winkler, 1996; Gray and Winkler, 2003) and are capable of being oxidized by compound I with concomitant formation of $\operatorname{Trp} 107^{\circ+}$ and Tyr $229^{\circ+}$ radicals. Simultaneous formation of these protein radicals from a single turnover event makes possible their coupling to the Tyr-Trp bond. Formation of a second compound I intermediate results in further oxidation of the Tyr-Trp cross link leading to nucleophilic attack of the sulfur atom of Met255 on the quinine-like intermediate, ultimately forming the Met-Tyr-Trp cross link (Ghiladi et al., 2005). A similar process for Tyr-Trp cross link formation has been proposed for Met255 variant of MtKatG but in this variant further reaction with oxidized Tyr-Trp was not possible (Ghiladi et al., 2005a). Mutating 
equivalent Trp to Ala in SyKatG did not yield any Met-Tyr-Trp cross link (Jakotipsch et al., 2003) which supported a sequential formation of bond between Tyr229 and Trp108, followed by Tyr229 and Met255.

The studies of Obinger and coworkers on the ferric, ferrous and fluoride complexes of Tyr249Phe, Met275Ile and Trp122Ala and Trp122Phe (SyKatG numbering) variants of SyKatG showed no significant effect on the protein structure on the proximal side of heme and attributed this to no change in frequency for the Feimidazole stretching modes as compared to the wild type SyKatG (Heering et al., 2002; Santoni et al., 2004; Santoni el al., 2004a). Tyr249Phe and Met275Ile variants showed similar ferric spectra as that of wild type with a tendency to loose heme which was attributed to the presence of a shoulder at $368 \mathrm{~nm}$ in conjugation with an increase of a 6coordinated (c) low spin (LS) heme at the expense of a native 5-coordinated high spin (HS) heme (Heering et al., 2002). The Trp122Phe and Trp122Ala variants exhibit increased $p K a$ of the alkaline transition and showed the presence of aquo 6-coordinated HS heme and hydroxyl 6-coordinated HS heme (Santoni et al., 2004a). These authers demonstrated weakened heme binding with a destabilized protein when the crosslink was only partially formed, but the absence of the cross-link did not alter the disposition of the distal the Trp because the binding of fluoride was unaffected compared to native protein. On the other hand the variants Trp122Phe and Trp122Ala affected the protein to bind fluoride (Smulevich et al., 2006).

A similar characterization of the equivalent variants (Tyr229Phe and Met255Ile) of MtKatG by Magliozzo and coworkers demonstrated spectroscopic features slightly different from those reported for SyKatG. The Met255Ile variant of MtKatG showed 
greater relative population of 6-coordinated HS heme than either native KatG or Tyr229Phe variant and all three (native MtKatG and the variants Tyr229Phe and Met255Ile) lacking a detectable LS heme component (Chouchane et al., 2003).

Jakopitsch et al demonstrated that KatGs can be converted into monofunctional peroxidases by mutating Tyr249Phe (in case of SyKatG). The variant Tyr249Phe also demonstrated the formation of an oxoferryl-porphyrin radical compound I upon addition of equimolar $\mathrm{H}_{2} \mathrm{O}_{2}$ to the ferric enzyme (a phenomenon difficult to observe in native enzyme due to its high catalase activity), followed by rapid formation of an intermediate with a oxoferryl type compound II spectrum similar to HRP (Jakopitsch et al., 2003). Auther attributed this spectrum to the result of an endogenous electron transfer similar to a kinetic coupling demonstrated between the generation of a tyrosyl radical and the formation of the intermediate with the compound II like spectrum in Tyr229Phe variant of MtKatG (Yu et al., 2003). This was also confirmed by sequential mixing-stopped flow analysis which directly produced oxoferryl type compound II by one electron reduction of the ferryl-porphyrin radical compound I (Jakopitsch et al., 2003). In the presence of micromolar concentration of $\mathrm{H}_{2} \mathrm{O}_{2}$ and in the absence of one-electron donors, a compound III like spectrum has also been reported in Tyr249Phe variant of SyKatG (Regelsberger et al., 2001; Jakopitsch et al., 2003). These reports support the conjecture that covalent cross linking of distal Met-Tyr-Trp plays a significant role in maintaining the catalase and peroxidase activities of KatGs. 


\subsection{Perhydroxy modification of Heme}

The second unusual modification is evident only in the BpKatG but not in the other three $\mathrm{KatG}$ structures. This is a perhydroxy modification on the ring I vinyl group of the heme (Carpena et al., 2003). A simple hydration like addition of hydrogen peroxide across the double bond of the vinyl group has been suggested as the reason for this modified heme (Carpena et al., 2003) [Figure 1.6b]. This modification on heme is shown to be removed easily by treatment of the enzyme with INH (X. Carpena, personal

communication). The function of the perhydroxy group could be to serve as a reservoir of $\mathrm{H}_{2} \mathrm{O}_{2}$ in the active site, available for the immediate reaction when a peroxidatic substrate is contacted (Carpena et al., 2003).

\subsection{Perhydroxy modification on the Indole of Trp111}

A perhydroxy modification on the indole nitrogen atom of Trp 111 was reported in the crystal structure of the Ser324Thr variant of BpKatG (Carpena et al., 2005).

Interestingly, the modified Trp111 was not observed in the first crystal structure report of the wild-type BpKatG (Carpena et al., 2003) but was present in a recent report and has been shown to be associuated the change in $\mathrm{pH}$ (Carpena et al., 2006). The crystals of BpKatG soaked at different $\mathrm{pH}$ values showed almost no extraneous electron density in the vicinity of the indole nitrogen at $\mathrm{pH} 4.5$ and almost $100 \%$ occupancy when $\mathrm{pH}$ was raised to 8.5 , suggesting the gradual appearance of modification from $0 \%$ occupancy to almost 100\% occupancy (Carpena et al., 2006). EPR spectra were also correlated with the presence of the Trp 111 modification at $\mathrm{pH}$ above 7 . The rapidity of perhydroxy-indole formation, $100 \%$ yield after just 1 min soaking of the crystal at $\mathrm{pH} 8.5$, suggests a very 
facile reaction with molecular oxygen in the buffer at higher $\mathrm{pH}$, which correlates with the higher $\mathrm{pH}$ of NADH oxidase reaction (Singh et al., 2004). The explanation for the presence of perhydroxy modification in the Ser324Thr variant at $\mathrm{pH} 5.7$ has been attributed to an inductive effect of the extra methyl group of Thr324 facilitating indole oxidation (Carpena et al., 2006).

\subsubsection{Presence of three large loops in the catalytic region of KatGs}

Apart from having a deeply buried heme, a longer and more constricted proposed access route for $\mathrm{H}_{2} \mathrm{O}_{2}$ and an unusual Met-Tyr-Trp covalent adduct, KatGs also have a short stretch and three large loop (LL) insertions (LL1-LL3) which differs them from peroxidases (Yamada et al., 2002). The short stretch occurs at position 102-113 with the sequence pattern 'SQXWWPADXGXY' on the distal side of the heme, and is highly conserved (>95\%) among all known KatGs (Zàmocký et al., 2001). It is positioned in the

region homologous to the connection between helices $\mathrm{A}$ and $\mathrm{B}$. The first large insertion, which is of variable length among KatGs, occurs between positions 210 and 251 with a length of $36-43$ residues between helices $D$ and $E$ situated between the distal and proximal catalytic domains. There is a conserved sequence on the C-terminus of this loop comprising residues 'AXXMGLIYVN'. The second large insertion at positions 300-334 between helices $F$ and $G$ has the same length for all known KatGs (35 or 36 residues) and is, relatively conserved among all KatGs with a signature sequence 'GXXPXXAXXEXQGLGW'. The third large insertion between residues 357 and 392 (between helices $\mathrm{G}$ and $\mathrm{H}$ ) seems not to be highly conserved among KatGs on the other hand there exist a similar although shorter insertion in cytochrome $c$ peroxidase. The 
conservation evident in the inserts suggests that the first and the second insertions as well as the short distal stretch are of functional importance for KatGs (Zàmocký et al., 2001). Recent crystal structure analysis of KatGs supports this hypothesis. Constriction of the access channel has been attributed to the LL1 and LL2 insertions, as LL2 is positioned at one edge of the heme between helices $\mathrm{D}$ and $\mathrm{E}$ connecting the distal and proximal catalytic domains. The LL1 insertion contains a number of residues neighboring the covalently linked Tyr249 that are highly conserved in KatGs viz Ile248, Asn 152, Pro252 and Glu253 (Jakopitsch et al., 2005). Asp152 has been reported to be hydrogen-bonded to the LL1 residue Ile248 providing stability to the heme architecture and its substitution markedly changes the proximal His291-Asp402 hydrogen bond interaction (Santoni et al., 2004), underlining the role of the LL1 insertion in linking the distal and proximal heme sites.

\subsubsection{Substrate access channel}

The crystal structure analysis of HmKatG (Yamada et al., 2002) BpKatG (Carpena et al., 2003), SyKatG (Wada et al., 2002), MtKatG (Bertrand et al., 2004), and the BpKatG Ser324Thr variant (Deemagaran et al., 2005) has revealed several KatG specific peculiarities distinguishing these enzymes from other heme peroxidases. These peculiarities have also been shown to play an important role in the substrate access to the active site. An access route, for the substrate hydrogen peroxide, to the distal side of the heme through a pronounced funnel shaped channel positioned similarly to, but longer and more constricted than the access route of monofunctional peroxidases, has been proposed (Carpena et al., 2003). This channel is narrowest near the residues Ser324 and Asp141, 
about $14 \AA$ from the heme iron atom. The constriction of the access channel results from the LL1 and LL2 insertions which are lacking in both ascorbate peroxidase and cytochrome $c$ peroxidase. Hydrogen peroxide entering the distal side cavity of BpKatG through the constricted portion of the channel will immediately come into contact with the active-site residues Arg109, Trp111 and His112 leading to generation of compound I in both the catalatic and peroxidatic modes of reaction, or for reduction of compound I in the catalatic reaction (Carpena et al., 2003). Most peroxidases have a second access route, approximately in the plane of the heme, leading to the distal heme cavity, but the equivalent route in $\mathrm{KatGs}$ is blocked by loops in this larger protein.

However, there is another funnel-shaped access channel that approaches a small central cavity containing a single metal ion, which, in BpKatG, was tentatively identified as sodium (Carpena et al., 2003). This second channel provides direct access to the core of the protein and to a region that encompasses two other structural features with possible functional significance. The first is a large cleft between the two domains and could be a potential substrate binding site with a clear intra subunit pathway for electron transfer to the heme through the Met-Tyr-Trp adduct. A second feature is that the side chain of Arg426, which can exist in two orientations and the predominant conformation $(>70 \%)$ differs from that in $\mathrm{HmKatG}$.

Contributing to the complexity of this region are two conformations of the side chain of Thr1 19, of which the minor $(<30 \%)$ is similar to the conformation in HmKatG. A co-ordination between the conformations of the Arg426 and Thr1119 side chains has been suggested (Carpena et al., 2003). Changes in the structure of BpKatG relative to HmKatG (the single ion, rotated Asp427 carbonyl group, and the displaced Thr119 and 
Arg 426 side-chains) are in close proximity to one another, are associated directly or indirectly through hydrogen bonds or ionic interactions, and represent the only changes in structure relative to $\mathrm{HmKatG}$ in this region of the protein. This correlation suggests that the changes may be functionally related, and that the region of the protein may have an, as yet undefined function. This possibility is reinforced by the fact that there is a region of undefined electron density located in the cavity vacated by the Arg 426 side chain in direct contact with the oxygen atom of the side chain from Tyr238, the central residue of the covalent cross link (Carpena et al., 2003).

Although, the main access channel in KatGs is more restricted compared to the monofunctional peroxidases, it is not as pronounced as in monofunctional catalases. Similar to monofunctional catalases, there are conserved acidic amino acids in the main access channel: Glu242 at the entrance of the channel and Asp141 at the 3-4 $\AA$ wide constriction in the access channel. Exchange of both residues significantly reduced the catalase, but did not alter the peroxidase activity, suggesting that they are critical for stabilizing the solute matrix in the channel and possibly for orienting the water dipoles. In addition, Asp141 controls the access to the distal residues Arg108, His112 and Trp111, an indication that it may also participate in actual oxidation of $\mathrm{H}_{2} \mathrm{O}_{2}$ to molecular oxygen (Jakopitsch et al., 2004).

Recently Jakopitsch et al. (2005) have reported the involvement of LL1 specific residues (Ile248, Asn251, Pro252, and Glu253) in the catalysis of SyKatG based upon their exposure to the solute matrix of the access channel. These authers proposed the existence of a very rigid and ordered structure built up by interactions of LL1 residues with both distal and proximal residues and the heme itself. Mutation Ile248Phe 
significantly altered the heme pocket similar to one involving Asp152Ser leading to the alteration of the hydrogen bonding between Ile248 and Asp152 and subsequent reduced catalase activity by $87 \%$, similar to Asp152Ser variant [97\%] (Jakopitsch et al., 2003). They also reported that mutation at Ile 248 brings changes in the proximal heme site mediated by LL1 and its connection with helices $\mathrm{E}$ and F, with helix F accommodating the proximal histidine. Mutations at Asn251 and Pro252 affected the coordination of the iron atom, although the variations were less pronounced and RR spectra were very similar to those of native SyKatG in both ferric and ferrous state. Mutations at Glu253, which is located at the entrance of the funnel shaped access channel $\sim 9 \AA$ from the constriction formed by Asp 152 and Ser335, did not alter the spin and coordination state of the enzyme but altered the bifunctionality of the enzyme by significantly reducing the catalase activity. From the crystal structures of $\mathrm{BpKatG}$ and $\mathrm{MtKatG}$, it is known that both Asn251 and Glu253 are connected by a hydrogen bond between the amide oxygen of Asn251 and the main chain nitrogen of Glu253 as well as via at least two water molecules in their vicinity and mutations at these residues changes and disrupts this hydrogen bonding network proposed to be essential for the catalase activity of KatGs (Jakopitsch et al., 2005). These reports suggest that in KatGs, compound I reduction (not formation) depends strongly on a defined and oriented water matrix maintained by the channel architecture and highly conserved residues at the KatG specific loop LL1 (Jakopitsch et al., 2005). 


\subsubsection{4. $\mathrm{pH}$ induced changes in catalase-peroxidases}

BpKat $\mathrm{G}$ exhibits different $\mathrm{pH}$ optima for three different activities, $\mathrm{pH} 5.5$ for peroxidase activity, pH 6.5 for catalase activity and $\mathrm{pH} 8.75$ for oxidase activity (Singh $e t$ al., 2004 and see below). Loewen and coworkers have recently demonstrated pH induced changes in the structure of BpKatG which might be playing a key role in modulating different activities in KatGs (Carpena et al., 2005; Carpena et al., 2006). A conserved arginine at position 426 in $\mathrm{BpKatG}$, situated about $\pm 20 \AA$ from the active-site heme, has been shown to act as a molecular switch moving between two conformations, the relative proportions of which vary among the different crystal structures. In one position, the guanidinium group forms an ionic association with Tyr238 in the Trp-Tyr-Met adduct [conformation Y], and in the second position, it is shifted to a region containing two other arginine residues [conformation R] (Carpena et al., 2005). The proportion of conformation $Y$ varies form about $0 \%$ in MtKatG (Berthand et al., 2004), to 30\% in BpKatG (Carpena et al., 2003), to $80 \%$ in SyKatG (Wada et al., 2002) and 100\% in HmKatG (Yamada et al., 2002), presenting the striking correlation with the $\mathrm{pH}$ values of crystallization, pH 4.5 for MtKatG, pH 5.6 for BpKatG, pH 6.3 for SyKatG and pH 8.1 for HmKatG. The apparent correlation between the conformation of Arg426 and the $\mathrm{pH}$ of crystallization was tentatively confirmed by increasing the $\mathrm{pH}$ for a crystal of $\mathrm{BpKatG}$ to 8.0 causing an increase in the amount of conformation $\mathrm{Y}$ to about 100\% (Carpena et al., 2005). This apparently facile movement of the side chain of Arg 426 coupled with its key role in catalase activity suggested that it can act as a molecular switch reversibly interacting with the Trp-Tyr-Met adduct to inductively alter heme reactivity. Another pH induced change observed in $\mathrm{BpKatG}$ was a perhydroxy modification on the indole 
nitrogen of $\operatorname{Trp} 111$, situated on the distal side of the heme which increases from $0 \%$ at pH 4.5 to $100 \%$ at $\mathrm{pH} 8.5$, correlating with the NADH oxidase activity (Singh et al., 2004). The covalent attachment of oxygen to the indole creates a very different molecular species compared to its noncovalent bonding to heme iron in other oxygen binding proteins. The fact that NADH is too large to fit into the heme cavity necessitates electron transfer from a remotely bound $\mathrm{NADH}$ to the oxygen most likely through protein, and covalent linkage of the oxygen to the indole makes possible direct electron transfer from the protein. The perhydroxy modification was detected by both X-ray crystallography and EPR spectroscopy (Carpena et al., 2006).

The $\mathrm{pH}$ induced shift from conformation $\mathrm{R}$ to $\mathrm{Y}$ of Arg426 can be correlated with and explains at least in part, the $\mathrm{pH}$ optima for the peroxidase, catalase, and NADH oxidase reactions of 4.5,6.5, and 8.75, respectively (Singh et al., 2004). At pH 4.5, Arg is predominantly in $\mathrm{R}$ conformation, remote from Tyr238, allowing the tyrosinate charge to delocalize onto the heme, enhancing oxyferryl formation and peroxidase reaction (Carpena et al., 2005). The equal proportions of $Y$ and $R$ at $\mathrm{pH} 6.5$ suggests a facile equilibrium providing optimum conditions for both oxyferryl formation (conformation $\mathrm{R}$ ) and oxyferryl reduction (conformation $\mathrm{Y}$ ) by $\mathrm{H}_{2} \mathrm{O}_{2}$ required for the catalase reaction. At pH 8.5, Arg426 occupies predominantly conformation $Y$, associated with Tyr238, which inductively lowers electron density on the heme and adduct favoring perhydroxy-indole reduction in the oxidase reaction (Carpena et al., 2006) 


\subsubsection{Protein radicals in catalase-peroxidases}

Electron transfer reactions between metalloproteins play critical roles in many important biological processes. However these reactions are poorly understood due to their dependence on so many factors, including the kinetics of complex formation and dissociation, the electronic properties of the redox centers, the distance and the pathway for electron transfer, and the driving force and the reorganization energy (Millett et al., 1995). One such widely studied reactions is that of cytochrome $c$ peroxidase with cytochreome $c$, in which the electron transfer pathway extends form the heme methyl group on cytochrome $c$ through CcP residues Ala194, Ala193, and Gly192 to the indolyl radical on Trp191, which is in van der Waals contact with the heme (Pelletier and Kraut, 1992). This tryptophanyl radical, exchange-coupled with the oxyferryl heme iron $\left[\mathrm{Fe}(\mathrm{IV})=\mathrm{O} \operatorname{Trp}^{\mathrm{o}^{+}}\right]$is necessary for the electron transfer from cytochrome $\mathrm{c}$ to the heme, because cytochrome $c$ can not directly access the $\mathrm{C} c \mathrm{P}$ heme active site (Pelletier and Kraut, 1992). This was identified as the relevant intermediate acting as an electron sink for the substrate (cytochrome $c$ ) oxidation in $\mathrm{CcP}$ (Sivaraja et al., 1889).

In lignin peroxidase, a tryptophanyl radical located close to the enzyme surface has been identified as the oxidation site for veratryl alcohol (Smith and Veitch, 1998; Sollewiyn et al., 2002). The formation of an oxyferryl-tyrosyl radical intermediate $\left[\mathrm{Fe}(\mathrm{IV})=\mathrm{O} \mathrm{Tyr}{ }^{\circ}\right]$ by means of intramolecular electron transfer between the heme and a tyrosine residue has been observed in catalases (Ivanicich et al., 1997), peroxidases (Ivanicich et al., 2001; Ivanicich et al., 2001a) and prosthaglandin H synthase (Tsai et al., 1999; Shi et al., 2000). 
Formation of such radicals on specific Tyr and/or Trp strongly favors their role as an alternate oxidation site, other than heme, in both catalases and peroxidases. In catalase-peroxidases, the exact nature and positions of such protein based radicals is poorly understood. Different groups are investigating the catalatic intermediates of KatGs. Hillar et al., 2000, attributed the observed 9-GHz EPR signal of the Compound I intermediate in EcKatG to an oxoferryl-porphyrin radical [Fe(IV)=O $\mathrm{Por}^{\mathrm{o}^{+}}$]. Magliozzo and coworkers in their study on MtKatG assigned an EPR signal to a tyrosyl radical (Chouchane et al., 2002) and later, using nitric oxide as a radical scavenging reagent, identified Tyr353 to be the radical site (Zhao et al., 2004). Interestingly, the residue at this position is conserved as Trp in all other KatGs used in the current study (Figure 4.2). The side chain of Tyr353 lies in the interior of MtKatG on the proximal side and close to the heme suggesting that Tyr 353 could be a site for radical formation, although proximity alone can not be the sole criterion for an electron transfer reaction pathway between Compound I and a Tyr residue (Zhao et al., 2004). The authors did not rule out formation of radicals at other residues and this remains the subject of debate (Ivanicich et al., 2003). The atypical formation of compound I intermediate $\left[\mathrm{Fe}(\mathrm{IV})=\mathrm{O} \mathrm{Por}^{\circ+}\right]$ as well as two subsequent protein based radical intermediates indentified as $\operatorname{Tyr}^{\circ}$ and $\operatorname{Trp}^{\mathrm{o}^{+}}$were identified in Synechocystis PCC6803 catalase-peroxidase of catalase and peroxidases, using a combination of multifrequency EPR spectroscopy, isotope labeling and site directed mutagenesis. The position of the $\operatorname{Trp}^{\circ}$ was assigned to $\operatorname{Trp} 106$ (SyKatG numbering) located on the distal side of the heme belonging to the highly conserved short stretch of KatGs. Existence of an extensive hydrogen bonding network on the distal side of the heme, involving Trp122, His123, Arg119, seven structural waters, and the heme 6- 
propionate group has been identified as a possible path for electron transfer from the tryptophanyl radical (Ivanicich et al., 2003). This notion was further supported (Jakopitsch et al., 2006) in a demonstration that the variant Trp122His (thus maintaining the hydrogen bond network) allowed the radical formation but the Trp122Phe mutation did not.

\subsubsection{Catalase-peroxidase from different organisms}

The complete sequences are known for catalase-peroxidases from various prokaryotes, both eubacteria and archaea. The systematic analysis of KatGs reveals that these enzymes are distributed unequally among closely related genomes whereas in some complete genomes, no KatG is present (such as Bacillus subtilis). Some bacteria even contain two different ones (e.g. Mycobacterium fortuitum) (Zámocký, 2004). In my study I studied catalase-peroxidase from: Burkholderia pseudomallei, Mycobacterium tuberculosis, Escherichia coli, Synechocystis PCC6803, Archaeoglobus fulgidus, Bacillus stearothermophilus and Rhodobacter capsulatus.

\subsubsection{Catalase-peroxidase of Burkholderia pseudomallei (BpKatG)}

B. pseudomallei (formally known as Pseudomonas pseudomallei), the causative agent of melioidosis, is a gram-negative soil bacillus endemic mainly in Southeast Asia and northern Australia, although cases have been reported in India, China, Taiwan, and Laos (Cheng and Currie., 2005). The disease may manifest itself in an acute, subacute, or chronic form; and the acute form of melioidosis is often fatal despite aggressive antibiotic treatment (Chen et al., 2004). In this era of biological weapons, B. pseudomallei is being 
increasingly recognized as a potential biological weapon (Leelarasamee, 2004). At a cellular level, this gram-negative bacterium can survive and multiply in both phagocytic and non-phagocytic cells and after internalisation, it can readily escape from the membrane-bound phagosome into the cytoplasm (Jone et al., 1996). The internalised $B$. pseudomallei can also induce a cell-to-cell fusion, resulting in multinucleated giant cell (MNGC) formation and cell death (Kespichayawattana et al., 2000). The rpoS null mutants of $B$. pseudomallei exhibits an increased sensitivity to oxidative stress (Subsin et al., 2003) and also demonstrate abnormal modulation of the host cell response, particularly with regard to the macrophage antimicrobial capacity to control the intracellular fate of B. pseudomallei (Utaisincharoen et al., 2006). There is also mention of a nonspecific DNA-binding protein (DpsA) whose expression increases in response to oxidative stress through increased transcription from the upstream kat $G$ (catalaseperoxidase) promoter, which is OxyR dependent (Loprasert et al., 2004).

Catalase-peroxidase of $B$. pseudomallei (BpKatG) is one of the antioxidant enzymes that are part of the oxidative stress response (Loprasert et al., 2003). The nucleotide sequence of $k a t G$ gene appeared in 2002 (Loprasert et al., 2002), and this was followed by the purification and crystallization of the enzyme (Carpena et al., 2002). BpKatG shows high sequence similarity to other catalase-peroxidases of bacterial, archaebacterial and fungal origin including $65 \%$ similarity to $\mathrm{KatG}$ from $M$. tuberculosis and lesser sequence similarity to members of plant peroxidase family (Carpena et al., 2002). In 2003, the $1.7 \AA$ crystal structure of $B p K a t G$ was reported (Carpena et al., 2003).demonstrating that $\mathrm{BpKatG}$ is a homodimer of $79 \mathrm{kDa}$ subunits with one modified 
heme group and one metal ion, likely sodium, per subunit. Each subunit has distinct Nand $\mathrm{C}$ - terminal domains that are structurally related and are similar to plant peroxidases.

\subsubsection{Catalase-peroxidase of Mycobacterium tuberculosis (MtKatG)}

First purified and described by Diaz and Wayne (1974), this enzyme attracted the attention of researchers because of the correlation of isoniazid resistance in Mycobacterium tuberculosis strains with loss of catalase and peroxidase activity in the cells (Winder, 1982). Following the characterization and cloning of the structural gene for the M. tuberculosis KatG (MtKatG) (Zhang et al., 1992), purification of the recombinant form of the catalase-peroxidase has been reported by a number of groups Nagy et al., 1995; Johnsson et al., 1997; Nagy et al., 1997a; Saint-Joanis et al., 1999; Young et al., 2003), and purification of KatG from M. intracellulare (Morris et al., 1992), M. smegmatis (Marcinkeviciene et al., 1995), M. fortuitum (Menendez et at., 1997), M. leprae (Eiglmeier et al., 1997), M. avium (Li et al., 2005), M. bovis (Garnier et al., 2003), M. canettii (Gutierrez et al., 2005) has also been carried out. Recent success in solving the crystal structure of catalase-peroxidases from Haloarcula marismortui (Yamada et al., 2002), B. pseudomallei (Carpena et al., 2003) and Synechococcus Pcc 7942 (Wada and Tada, 2003) was followed by the structure of M. tuberculosis KatG, MtKatG (Bertrand et al., 2004) and its Ser315Thr variant (Yu and Sacchettini, 2006), one of the key variants thought to be involved in $\mathrm{N} H$ resistance. 


\subsubsection{Catalase-peroxidase of Escherichia coli (EcKatG)}

E. coli has three different enzymes withj catalase activity and the monofunctional catalase HPII of E. coli has already been discussed in Section 1.4. The bifunctional catalase-peroxidase "hydroperoxidase I [HPI or EcKatG]" (Claiborne and Fridovich, 1979) and monofunctional catalase "hydroperoxidase II (HPII)" (Claiborne et al., 1979) are chromosomally encoded enzymes, both of which has been extensively studied (Chelikani et al., 2004). In 1996, a third enzyme from enterohemorrhagic E. coli O157:H7 was characterized as a periplasmic catalase-peroxidase called KatP (Brunder et $a l, .1996)$. KatP has a molecular mass of $81.8 \mathrm{kDa}$ and the presence of an $\mathrm{N}$-terminal signal sequence suggests that KatP is exported to and functions in the periplasm.

Production of KatP by highly virulent bacterial pathogens but not by their less pathogenic or non-pathogenic relatives, suggests that these enzymes may be used as virulence factors (Cornelius et al., 2003).

HPI or EcKatG is encoded by the kat $G$ gene located at 89.2 min on the $E$. coli chromosome (Loewen et al., 1985b). Originally it was described as a tetrameric enzyme made up of identical $80 \mathrm{kDa}$ subunits containing two heme $b$ prosthetic groups per tetramer (Claiborne and Fridovich, 1979). Later partial heme occupation of HPI like some other catalase-peroxidases was shown with an average of 0.5 heme per subunit in a heterogenous mixture of dimers and tetramers with $0,1,2$ and 3 hemes respectively (Hillar et al., 2000). EcKatG has a specific catalase activity of about $2000 \mathrm{U} / \mathrm{mg}$ (Loewen et al., 1990; Hillar et al., 2000) which is only about one seventh of that reported for the large subunit catalase HPII also from E. coli (Loewen and Switala, 1986). To date, 
success in crystallizing EcKatG remains elusive although the crystal structure of its $\mathrm{C}$ terminal domain has been published (Carpena et al., 2004).

\subsubsection{Catalase-peroxidase of Synechocystis PCC 6803 (SyKatG)}

Cyanobacteria represent one of the major phylogenetic lines of bacteria, comprising a large and heterogeneous group of phototrophic prokaryotes. It is widely accepted that they were the first oxygen-evolving organisms and were responsible for the conversion of the atmosphere of the earth from an anoxic to an oxic one. Microfossil evidence of cyanobacteria-like cells is well documented for the Precambrian era i.e. more than three billion years ago (Schopf, 1993), and there is evidence that cyanobacteria occupied large areas of the earth in those ancient times. The evolution of oxygenic photosynthesis had enormous consequences for the environment and survival was dependent on either occupying an anaerobic habitat or developing defense strategies against the reactive oxygen species, such as superoxide anion, hydrogen peroxide, and hydroxyl radical.

Cyanobacterial catalase-peroxidases have been subjected to extensive characterization. Mutsuda et al (1996) reported the purification and nucleotide sequence analysis of a catalase-peroxidase from Synechococcus PCC 7942, and Obinger et al (1997) described the characterization of a catalase-peroxidase from Anacystis nidulans

(Synechococcus PCC 6301). The enzymes from both cyanobacteria showed peroxidase activity with $o$-dianisidine and pyrogallol, but no reaction was detected with guaiacol, ascorbate, NADH or NADPH. They are homodimeric enzymes with a molecular mass of 
approximately $80 \mathrm{kDa}$ per subunit. Both are very efficient catalases with a $K_{\mathrm{m}}$ for $\mathrm{H}_{2} \mathrm{O}_{2}$ in the millimolar range.

\subsubsection{Catalase-peroxidase of Archaeoglobus fulgidus (AfKatG)}

Archaeoglobus fulgidus is a strict anaerobic hyperthermophilic archaeon that has been isolated from marine hydrothermal environments as well as subsurface oil fields. This sulfate reducer can grow organoheterotrophically with a variety of carbon sources, or lithoautotrophically on hydrogen, thiosulfate, and $\mathrm{CO}_{2}$ (Stetter, 1988). Besides its ability to grow at extremely high temperatures, this organism is unusual in that it is unrelated to other sulfate reducers. The sequencing of the entire genome of $A$. fulgidus was completed in 1997 (Klenk et al. 1997), which revealed the presence of an open reading frame that was putatively identified as a catalase-peroxidase on the basis of its high similarity ( $62.9 \%$ nucleotide identity; $49.5 \%$ amino acid similarity) to the per $A$ gene of Bacillus stearothermophilus (Klenk et al., 1997; Loprasert et al., 1989). The purified AfKatG is a homodimer with a subunit molecular mass of $85 \mathrm{kDa}$.

\subsubsection{Catalase-peroxidase of Bacillus stearothermopohilus (BsKatG)}

B. stearothermopohilus is Gram-positive bacteria found in soil, hot springs, Arctic waters, ocean sediments, and spoiled food products. BsKatG is a dimeric enzyme with 731 amino acids per subunit and has $48 \%$ homology with EcKatG. It has an optimum temperature of $70^{\circ} \mathrm{C}$ and is stable for a month at $30^{\circ} \mathrm{C}$ (Loprasert et al., 1997). 


\subsubsection{Catalase-peroxidase of Rhodobacter capsulatus (RcKatG)}

$R$. capsulatus is a photosynthetic, facultatively anaerobic bacterium that is capable of fixing molecular nitrogen. It has two hydroperoxidases: a peroxidase and a catalaseperoxidase which make it suitable as a test organism for studying oxidative damage and protection mechanisms (Hochman et al., 1991). R. capsulatus katG (cpeA) was initially sequenced incorrectly in 1993 (Forkl et al., 1991) and later corrected (Nestor Cortez personal communication).

\subsubsection{Catalase-peroxidase and isoniazid activation}

M. tuberculosis, the causative agent of tuberculosis (TB), is a tenacious and remarkably successful pathogen that has latently infected a third of the world's population. Each year there are eight million new TB cases and two million deaths (Zhang et al., 2006). The increasing emergence of drug resistant TB, and HIV infection, which compromises host defense and allows latent infection to reactivate or render individuals more susceptible to $\mathrm{TB}$, pose further challenges for effective control of the disease (Corbett et al., 2003).

Isonicotinic acid hydrazide (isoniazid or $\mathrm{INH}$ ) [Figure $1.7 \mathrm{~A}$ ] is one of the front line drugs used in the treatment of tuberculosis, but the mode of action of this 55 year old

drug is still poorly understood (Kapetanaki et al., 2005). $\mathrm{NH}$ is a prodrug that requires in vivo activation by KatG upon entering $M$. tuberculosis (Zhang et al., 1992). The need for KatG activation was deduced soon after the introduction of INH therapy when $\mathbb{N H}$ resistant strains were correlated with the loss of catalase-peroxidase activity (Middlebrook, 1952 and 1954). This observation was confirmed by the molecular and 
genetic studies of Zhang and colleagues which demonstrated that INH-sensitivity could be restored by the introduction of a functional kat $G$ gene into an INH-resistant, catalasedeficient strain of M. tuberculosis and in Mycobacterium smegmatis (Zhang et al., 1992 and 1993). This was followed by numerous reports demonstrating that mutation in, or deletion of, the $k a t G$ gene results in the acquisition of isoniazid resistance (Heym et al., 1995; Morris et al., 1995; Rouse et al., 1996; Saint-Joanis et al., 1999).

The second gene found in M. tuberculosis to be associated with INH resistance is inhA (Banerjee et al., 1994). InhA, the product of inhA, is an NADH-dependent enoyl-acyl carrier protein reductase. Mutations within the inhA structural gene (Ristow et al., 1995) or promoter (Kapur et al., 1995) have been identified and shown to be associated with both INH and ethionamide (ETH), a structural analog of the isoniazid, resistance (Larsen et al., 2002). Missense mutations within the inhA structural gene cause INH resistance by reducing the NADH [Figure 1.7B] binding affinity of InhA and thus protecting the enzyme from INH inactivation (Rozwarski et al., 1998). Formation of isonicotinoyl-NAD [Figure $1.7 \mathrm{C}$ ], the active form of the drug, involves loss of hydrazine $\left(\mathrm{N}_{2} \mathrm{H}_{2}\right)$ from $\mathbb{I N H}$ promoted by KatG (Zhang et al., 1992) and binding of the isonicotinoyl group to $\mathrm{NAD}^{+}$ (Johnsson et al., 1995). Isonicotinoyl-NAD mimics the NADH and binds to InhA [Figure 1.7D] (Rozwarski et al., 1998), and possibly to KasA, a $\beta$-ketoacyl acyl carrier protein synthase (Mdluli et al., 1998), blocking their NADH-binding sites and interfering with the cell wall synthesis.

The central role of KatG in $\mathbb{N H}$ activation is evident from the significant number of $\mathbb{N} H$-resistant cases of tuberculosis attributable to mutations in $\mathrm{kat} G$ and from biochemical studies that have demonstrated a direct role for $\mathrm{KatG}$ in the generation of 
various isonicotinoyl derivatives (Johnsson and Schultz, 1994). Much of the literature related to the activation of INH by $\mathrm{KatG}$ has focused on the fate of $\mathrm{INH}$ and possible intermediates involved in the process (Johnsson and Schultz, 1995; Wilming and Johnsson, 1999; Magliozzo and Marcinkeviciene, 1996). With NAD included in the mix, the generation of the isonicotinoyl-NAD adduct was observed both with and without KatG present (Wilming and Johnsson, 1999; Magliozzo and Marcinkeviciene, 1996; Lei et al., 2000), leading to the suggestions that the role of $\mathrm{KatG}$ is limited to the hydrazine lyase of INH and that the subsequent reaction of the isonicotinoyl radical with $\mathrm{NAD}^{+}$is a nonenzymatic event involving a homolytic aromatic substitution (Wilming and Johnsson, 1999; Minisci et al., 1989). Reactive oxygen species have also been implicated in $\mathbb{N H}$ activation both in vivo (Mitchison et al., 1989; Youatt 1969) and in vitro (Johnsson and Schultz, 1995), and an elevated level of intracellular superoxide (Wang et al., 1998) have also been associated with the high sensitivity of M. tuberculosis to INH (Deretic et al., 1996). However, the absence of $\mathrm{H}_{2} \mathrm{O}_{2}$ involvement in $\mathrm{INH}$ activation implies that a reaction different from either the peroxidase or the catalase reactions may be involved, and some reports have suggested that the active participation of KatG in $\mathbb{N H}$ activation involves more than just hydrazinolysis (Lei et al., 2000).

\subsubsection{KatG mutations involved in isoniazid resistance}

Analysis of patient isolates resistant to INH has led to the conclusion that a relatively minor subset entirely lacks catalase-peroxidase activity. Typically this subset of strains has deletions or frame-shifts in $k a t G$ and are resistant to high levels of $\mathbb{N H}$ (Payton et al., 1999). Small insertions, partial deletions, and missense mutations are 
<smiles>CN(N)C(=O)c1ccncc1</smiles>

A

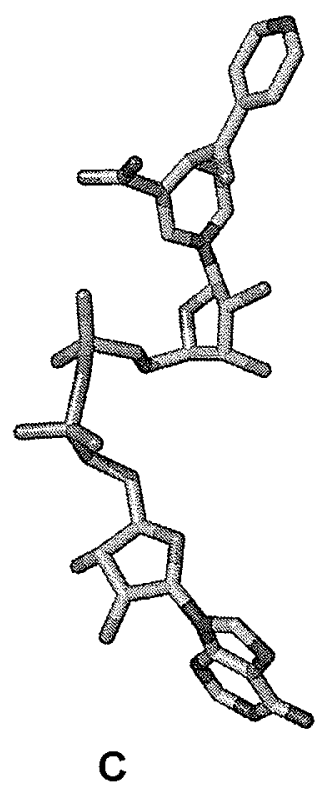

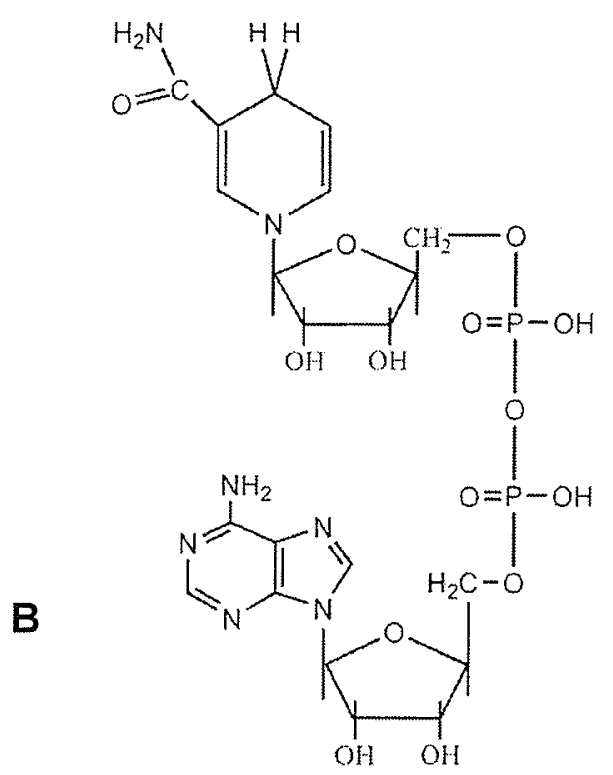

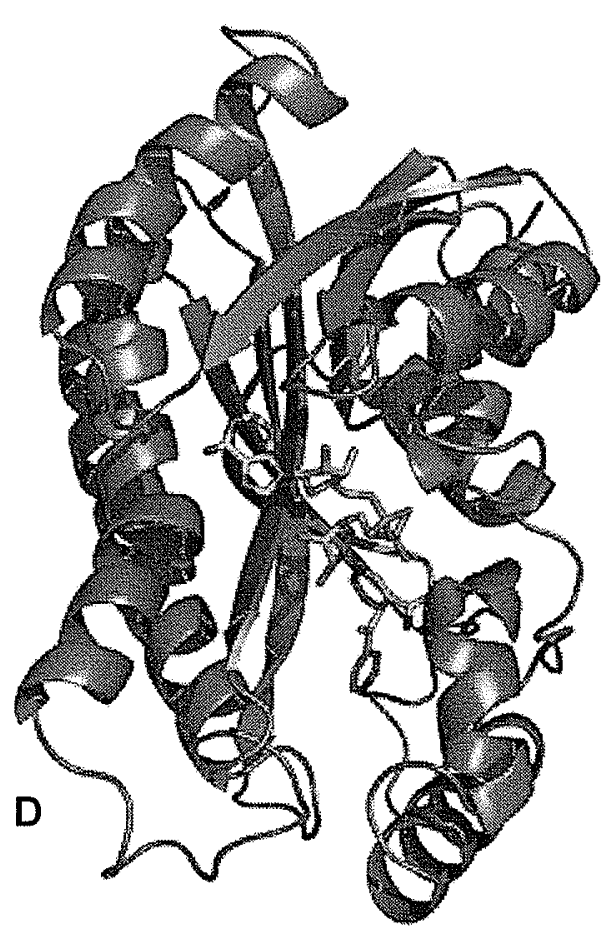

Figure 1.7. Structures of isoniazid (INH) (A), NADH (B), isonicotinoyl-NAD adduct (C) and isonicotinoyl-NAD adduct in bound form with InhA protein of M. tuberculosis (D). The structures were created using Chem Draw software and using Pymol (Deleno, 2003) and using the coordinates submitted to the protein data bank. 
observed at a low frequency in $\mathrm{NH}$-resistant clinical isolates. In contrast to these complete loss of function events, in vivo mutations in $k a t G$ that result in diminished or altered catalase-peroxidase activity are much more frequently observed amongst patient isolates (Victor et al., 1996; Heym et al., 1995; Kelley et al., 1997; Stoeckle et al., 1993; Altamirano et al., 1994).

Although, the real occurrence of these events in vivo is unknown, presumably these are more on the order of typical point mutation rates, occurring in perhaps one in $10^{8-9}$ organisms. The most commonly observed mutation is a point mutation replacing serine at location 315 with threonine (S315T). Estimates are that this mutation occurs in between 30 and $60 \%$ of all INH resistant isolates (Musser et al., 1996; Marttila et al., 1996; Martila et al., 1998). Studies on recombinant wild type MtKatG and the variant Ser315Thr demonstrated that the mutation results in somewhat reduced enzyme activity (Wengenack et al., 1997; Saint-Joanis et al., 1999; Rouse et al., 1996) but more recent studies on the Ser315Thr variant and its equivalent Ser324Thr in B. pseudomallei have shown that this mutation maintains catalase and peroxidase-specific activities similar to the native enzyme with only subtle differences among the kinetic parameters (Deemagarn et al., 2005).

The basis of the resistance of isoniazid became evident with the determination of crystal structures of different KatGs (Yamada et al., 2002; Carpena et al., 2003; Wada et al., 2002; Bertrand et al., 2004) and the crystal structure report of BpKatG variant Ser324Thr (equivalent of Ser315Thr) (Deemagaran et al., 2005). A calculated surface diagram reveals that the channel in native $B p K a t G$ adjacent to Ser324 is significantly wider than the channel in its variant Ser324Thr to the extent that a number of water 
molecules present in the native enzyme are absent from the variant. Consequently, it is interference with $\mathrm{NH}$ accessibility to the heme cavity that best explains the reduced affinity and reactivity of $\mathrm{KatG}$ towards $\mathrm{INH}$, although the possibility of INH binding in the channel cannot be excluded, in which case the methyl group of Thr324 would interfere with $\mathrm{NH}$ binding. Other mutations of Ser324 might cause a similar blockage of the channel, but the situation may be more complex because the hydrogen bond between the hydroxyl group of Ser (and Thr) and the propionate carbonyl group would disappear. The effect that this would have on the structural integrity of the enzyme, particularly in the channel region, and the electronic environment of the heme is not clear. For example, the complete lack of isonicotinoyl-NAD synthase activity and NH hydrazinolysis activity in the Ser315Asn variant of MtKatG may be a result of the larger Asn side-chain situated in the channel or of some other distortion in the subunit arising from the lack of interaction with the heme propionate group (Deemagarn et al., 2005).

Although $k a t G$ mutations resulting in Ser315Thr are the most common mechanism of $\mathrm{INH}$ resistance as determined by sequencing, additional mutations also occur in functionally important residues such as Ser315Asn, Asp63Glu, His108Gln, Thr262Arg, Ala350Ser and Gly629Ser. Biochemical characterization of some of these variant proteins has confirmed their importance to protein stability and catalytic properties. An amino acid polymorphism involving the change of Arg463 to Leu, was initially thought to be associated with INH resistance but couln't be associated with the INH resistance by biochemically or epidemiologically (Johnsson et al., 1997; Heym et al., 1995; Musser et al., 1996; Rouse et al., 1995; Pretorius et al., 1995; Lee et al., 1997; Nagy et al., 1998). 


\subsubsection{Analysis of the potential isoniazid binding site}

An area of wide dispute centers on the location of isoniazid binding to KatG although the recent success with the crystallization of KatG has provided some insight into this. The crystal structure report of BpKatG shows a region of undefined electron density located just before the constricted region in the main channel leading to the distal heme cavity of BpKatG over $10 \AA$ further away from the heme in close proximity to Ser324. This unidentified electron density was evident both in the structure obtained after a short soaking with $\mathbb{N H}$ and in the structure obtained without soaking and is thought to be the result of a metabolic constituent of E. coli, which may closely resemble INH such as pyridoxol or vitamin $\mathrm{B} 6$.

The location of this undefined electron density is the same in the crystal structures of native $\mathrm{BpKatG}$ and its variants (Deemagaran et al., 2005; unpublished data) and is consistence with the observation of a small peak of electron density in the same site in MtKatG (Bertrand et al., 2004). Bertrand et al argued this region to be too small to accommodate a pyridine-like metabolite and suggested it as solvent or a small hydrophobic molecule. Bertrand et al (2004) concluded that the binding site for aromatic compounds is near the $\delta$-meso edge of the heme based on a comparison with the crystal structures of monofunctional peroxidases from all three classes (APX with salicylhydroxamic acid (Sharp et al., 2004); Arthromyces ramosus peroxidase with salicylhydroxamic acid (Tsukamoto et al., 1999); HRPC with benzhydroxamic acid (Henriksen et al., 1998). 


\subsection{Objectives of the thesis}

Since the first report characterizing the purified catalase peroxidase HPI [EcKatG] of E. coli (Claiborn and Fridovich, 1979), KatGs have been purified and characterized from a large number of organisms. Even after almost three decades, there is still more to know about this fascinating enzyme. Two important issues that need to be addressed include the role of $\mathrm{KatG}$ in isoniazid activation against $M$. tuberculosis with specific interest in the drug protein interaction at the molecular level and the location of the binding site of $\mathrm{NH}$. Another key area is the reaction mechanism by which this enzyme maintains its bifunctionality, particularly the fate and role of the intermediates formed during the catalase and peroxidase reactions. The in vivo peroxidatic substrate(s) for KatGs need(s) to be identified so as to determine the physiological role for the peroxidatic activity of KatGs. The recent success in crystallization of KatGs is promising in understanding their structure function.

This study reports the comparison of catalase-peroxidases from seven different organisms, M. tuberculosis, B. pseudomallei, E. coli, B. stearothermophilus, A. fulgidus, Synechosystis PCC 6803, and $R$. capsulatus. The KatG gene product from these organisms was over expressed from the $\mathrm{katG}$ gene cloned in a suitable vector. The over expressed protein was purified and characterized for its biochemical and biophysical properties such as $\mathrm{pH}$ profile and enzyme kinetics. The major focus was on KatG mediated isoniazid activation, the formation of intermediates and subsequently the formation of isonicotinoyl-NAD adduct the active form of isoniazid. Two key mutations involved in isoniazid resistance, Ser315Thr and Ser315Asn, were also constructed, purified, characterized and compared with the native MtKatG for drug activation. 
2. MATEIALS AND METHODS

\subsection{Escherichia coli strains, plasmids and bacteriophage}

E. coli strains, plasmids and bacteriophage used in this study are listed in Table 2.1.

Phagemid $\mathrm{pKS}+$ - from Stratagene cloning system were used as a vector for mutagenesis, sequencing and cloning. The $M$. tuberculosis kat $G$ gene encoding $\mathrm{MtKatG}$, originally in pYZ55 (Zhang et al., 1992) was recloned as an EcoRV-KpnI fragment into pKS+ as pAH1 (Hiller, 1999). Two different sub-clones were constructed for the site-directed mutagenesis in M. tuberculosis katG. A 600bp EcoRV-ClaI fragment from pAH1 was sub-cloned into pKS+ to generate pMt-EC (Fragment I, Figure 2.1) and a 470bp XholClaI fragment from $\mathrm{pAH} 1$ was sub-cloned into $\mathrm{pKS}+$ to generate $\mathrm{pMt}-\mathrm{XC}$ (Fragment II, Figure 2.1). The B. pseudomallei kat $G$ gene, encoding catalase-peroxidase BpKatG, was inserted into plasmid pKS- to generate the plasmid pBpG (Deemagaran, 2004). For sitedirected mutagenesis purposes, $\mathrm{pBpG}$ was subcloned as $\mathrm{pBpG}-\mathrm{KH}$ and further subcloned as pBpG-CH, containing fragment II and I respectively of $B$, pseudomallei kat $G$ (Figure 2.2). Strain CJ236 was used to generate single stranded uracil containing DNA templates from $\mathrm{pMt}-\mathrm{EC}$, $\mathrm{pMt}-\mathrm{CX}$ and $\mathrm{pBpG}-\mathrm{CH}$. The E. coli kat $G$ gene encoding HPI (EcKatG) was cloned into a pBR322 derived plasmid to generate pBT22 (Triggs-rain and Loewen, 1987). The $A$. fulgidus $k a t G$ was cloned into a pET-28b+vector as pLUW640 (Kengen et al., 2001). The B. stearothermophilus kat $G$ was cloned as pBR322 (Loprasert et al., 1989) to over express BsKatG. The Synechocytis katG encoding SyKatG was cloned into pET-3a vector and over expressed in E. coli BL21- (DE3) pLysS (Jakopitsch et al., 1999). 
Table 2.1. Genotypes and sources of Escherichia coli strains, plasmids and bacteriophage used in this study.

\section{Genotype Source}

\section{E. coli strains}

CJ236

NM522

JM109

UM262

BL2 1 trxB(DE3) pLysS dut l ungl thi-1 relAl/pCJ105/ $\mathrm{cam}^{\mathrm{r}} \mathrm{F}^{2}$

sup $\mathrm{E} \Delta($ lac-pro $\mathrm{AB})$ hsd-5 [F'pro $\mathrm{AB}$ lacI $I^{q}$ lacZ $\left.\Delta 15\right]$

recAl supE44 endA1 hsdA1 hadR17 Yanisch-Perron et al., 1985 gyr 96 relA1 thi $\Delta$ (lac-proAB)

recA kat $G:: T n 10$ pro leu rpsL hsdM Loewen et al., 1990 hsd $\mathrm{R}$ end lac $\mathrm{Y}$

$\mathrm{F}^{-}$ompT hsdS $\mathrm{b}_{\mathrm{b}}\left(\mathrm{r}_{\mathrm{b}}{ }^{-} \mathrm{m}_{\mathrm{b}}{ }^{-}\right) \mathrm{gal} d \mathrm{dcm}$ $\operatorname{trx} B 15:: \operatorname{kan}(\mathrm{DE} 3)$ pLys $\left(\mathrm{Cm}^{\mathrm{R}}\right)$
Kunkel et al., 1987

Mead et al., 1985

Novagen

\section{Plasmids}

pKS-

pAHl(pSK+, M. tuberculosis kat $G$ clone)

pBpG (pKS-, B. pseudomallei kat G clone)

pBT22 (pBR322, E. coli katG clone)

pOD10 (pBR322, B. stearothermophilus kat $G$ clone)

pLUW640 (pET9d A. fulgidus kat G clone)

pET3a (Synechosystis PCC 6803 katG clone)

pHF100 ( $R$. capsulatus kat $G$ clone)

pMt-CX (pSK+, M. tuberculosis kat $G$ subclone I)

pMt-EC(pSK+, M. tuberculosis katG subclone I)

pBpG-KH (subclone I)

pBpG-CH (subclone II)

pEMt (pET28b ${ }^{+}$, M. tuberculosis kat $G$ clone)
Ampr $^{\mathbf{R}}$ Stratagene Cloning System

Ampr $^{\mathrm{R}}$ Hillar et al., 1999

Ampr $^{\mathrm{R}}$ Deemagarn M.Sc. thesis, 2004

Ampr $^{\mathbf{R}} \quad$ Triggs and Loewen 1987

$\mathrm{Ampr}^{\mathrm{R}} \quad$ Loprasert et al., 1997

$\mathrm{Kan}^{\mathrm{R}}$ Kengen et al., 2001

Ampr $^{\mathrm{R}}$ Jakopitsch et al., 1999

$\mathrm{Ampr}^{\mathrm{R}}$ Hochman et al., 1986

Ampr $^{R}$ This study

Ampr ${ }^{\mathrm{R}}$ This study

Ampr $^{\mathbf{R}}$ This study

Ampr ${ }^{R}$ This study

$\mathrm{Kan}^{\mathrm{R}}$ This study

\section{Bacteriophage}


Table 2.2a Sequence of oligonucleotides and restriction fragments used for the site directed mutagenesis of $M$. tuberculosis kat $G$

\begin{tabular}{llll}
\hline Primer & $\begin{array}{c}\text { Bases } \\
\text { changed }\end{array}$ & Oligonucleotide sequence & Restriction fragment \\
\hline Ser315Thr & AGC-ACC & GCGATCACCACCGGCATCG & ClaI-Xhol (590-1064) \\
Ser315Asn & AGC-AAC & GCGATCACCAGCGGCATCG & ClaI-XhoI (590-1064) \\
Trp321Phe & TGG-TTC & GAGGTCGTATTCACGAACACC & ClaI-XhoI (590-1064) \\
Trp91Phe $^{b}$ & TGG-TTC & CAGCCGTGGTTCCCCGCCGAC & EcorV-ClaI (1-490) \\
\hline
\end{tabular}

Table 2.2b Sequence of oligonucleotides and restriction fragments used for the site directed mutagenesis of $B$. pseudomallei kat $G$

\begin{tabular}{lcll}
\hline Primer & $\begin{array}{c}\text { Bases } \\
\text { changed }\end{array}$ & Oligonucleotide sequence $^{\mathrm{a}}$ & Restriction fragment $^{\text {Pad }}$ \\
\hline His279Asn $^{\mathrm{b}}$ & CAC-AAC & CGCGGCGGCAACACGTTCGGC & $\begin{array}{l}\text { ClaI-HindIII (990- } \\
1710)\end{array}$ \\
His279Tyr $^{\mathrm{b}}$ & CAC-TAC & CGCGGCGGCTACACGTTCGGC & $\begin{array}{l}\text { ClaI-HindIII (990- } \\
1710)\end{array}$ \\
\hline
\end{tabular}

Table 2.2c Primers used for the polymerase chain reaction for the cloning of $k a t G$ from M. tuberculosis into $\mathrm{pET} 28 \mathrm{~b}+$ vactor.

\section{Primer name Oligonucleotide sequence}

\begin{tabular}{lll}
\hline I8NT9S $^{b}$ & AACACCCACCGAATTCAGAAACCAC & EcoRI \\
MtKatG-2 $^{b}$ & CGACTAATTCGAACTAGCC & HindIII
\end{tabular}

a The sequences in bold are the codons that have been modified.

${ }^{\mathrm{b}}$ Results from the variants His279Asn, His279Tyr, Trp321Phe, Trp91Phe and $M$. tuberculosis kat $G$ gene cloned in the vector pET28b+ have not been reported in the thesis. 
express cyanobacterium catalase-peroxidase in E.coli (Jakopitsch et al., 1999).

Rhodobactor capsulatus catalase-peroxidase was cloned as pHF100 in pBlusescript (Forkl et al., 1992). Helper Phage R408 was used for the infection of the strain CJ236 to generate single-stranded DNA. Strain NM522 was used for cloning and plasmid propagation. Strain JM109 was used for the production of plasmid DNA for double stranded DNA sequencing. Strain UM262, an E.coli strain with a kat $G$ gene interruption, was used for routine expression of $\mathrm{KatG}$ from various plasmids. Genes cloned on $\mathrm{pET}$ expression vectors were expressed in E.coli strain BL21(DE3) pLysS.

\subsection{Biochemical and reagents}

All biochemicals and reagents, as well as antibiotics used in the course of these studies were purchased from either Sigma Chemical Company (St. Louis, Missouri), or from Fischer Scientific Limited (Mississauga, Ontario). Restriction endonucleases, polynucleotide kinase, T4 DNA ligase and most of other enzymes used in these studies were purchased from Invitrogen Canada Inc. (Burlington, Ontario). The T7 sequencing kit was purchased from USB Corporation (Cleveland, Ohio). Components of the media used for growth of bacterial cell cultures were purchased from DIFCO, U.S.A. Reverse osmosis distilled water was used in preparing all solutions.

\subsection{Media, growth conditions and storage of cultures}

E. coli cultures were routinely grown in LB (Luria-Bertani) medium containing $10 \mathrm{~g} / \mathrm{L}$ Tryptone, $5 \mathrm{~g} / \mathrm{L}$ Yeast Extract and $5 \mathrm{~g} / \mathrm{L} \mathrm{NaCl}$. Solid LB medium contained $15 \mathrm{~g} / \mathrm{L}$ agar. For strains harboring plasmids bearing the ampicillin resistance gene, ampicillin was added to a concentration of $100 \mu \mathrm{g} / \mathrm{mL}$ for maintenance of selection pressure while 
growing in both liquid and solid LB medium. For induction of expression, cells were grown for $2-3 \mathrm{~h}$ until the absorbance at $600 \mathrm{~nm}$ reached $\sim 0.6$ and then $1 \mathrm{mM}$ IPTG was added for induction. In addition, chloramphenicol was added to a concentration of $40 \mu \mathrm{g} /$ $\mathrm{mL}$, in order to maintain the presence of $\mathrm{F}^{\prime}$ episome for the growth of the strain CJ236. E.coli strains in general were grown in solid media at $37^{\circ} \mathrm{C}$. However, cell cultures in liquid media for expression of wild type and variant proteins were grown at either $28^{\circ} \mathrm{C}$ or at $37^{\circ} \mathrm{C}$ in shake flasks with good aeration depending what was optimum for a particular variant. Bacterial strains were stored at $-60^{\circ} \mathrm{C}$ in $24 \%$ dimethylsulfoxide (DMSO). Bacteriophage R408 was stored at $4^{\circ} \mathrm{C}$ in LB culture supernatant.

\subsection{DNA manipulation}

\subsubsection{Preparation of synthetic oligonucleotides}

Oligonucleotides used for mutagenesis were purchased from Invitrogen (Burlington, Ontario) in non-phosphorylated form. The concentration of oligonucleotide DNA was determined spectrophotometrically at $260 \mathrm{~nm}$, where 1 absorbance unit $=40$ $\mu \mathrm{g} / \mathrm{mL}$ single strand DNA (Sambrook et al., 1998). Oligonucleotides used for the sitedirected mutagenesis were phosphorylated at the $5^{\prime}$ end using $T_{4}$ kinase (Invitrogen) according to Ausubel et al. (1989). Approximately $100 \mathrm{ng}$ of oligonucleotide DNA in a volume of $25 \mathrm{~mL}$ containing $1 \mathrm{mM} \mathrm{ATP}$ and 10 units of kinase were incubated at $37^{\circ} \mathrm{C}$ for $30 \mathrm{~min}$ in appropriately diluted buffer supplied by the manufacturers. The reaction was terminated by heat inactivation at $65^{\circ} \mathrm{C}$ for $5 \mathrm{~min}$. 


\subsubsection{Site-directed mutagenesis strategy}

Targeted base changes on phagemid-borne M. tuberculosis kat $G$ and $B$. pseudomallei kat $G$ were generated according to the in-vitro mutagenesis protocol described by Kunkel et al. (1987). Sub-clones were constructed from parts of the chromosomal insert containing the $k a t G$ gene on $\mathrm{KS}(+/-)$ vector. Simplified restriction maps of $M t k a t G$ gene and Bpkat $G$ gene indicating the location of individual sub-clones are shown in Figures 2.1 and 2.4, respectively. The sub-clone rather than the whole gene was mutagenized in order to limit the amount of subsequent sequencing required to confirm the mutation in the subclone both after mutagenesis and after reinsertion into $M t k a t G$ and Bpkat $G$ gene for protein expression. Target codons for mutagenesis were selected from the DNA sequence of $M t k a t G$ and $B p k a t G$ gene shown in the Figures 2.3 and 2.6, respectively. DNA sequences for oligonucleotides used in the mutagenesis procedure are listed in Table 2.2. Mutagenesis was performed by annealing the phosphorylated oligonucleatides encoding the desired base modifications to uracil containing single strand DNA templates obtained from the appropriate Bluescript phagemid sub-clone. The complementary DNA strand was then synthesized in vitro by unmodified T7 DNA polymerase (New England Biolabs) using the annealed oligonucleotoide as a primer. The $3^{\prime}$ and $5^{\prime}$ ends of the completed complementary strand were ligated by adding T4 DNA ligase (Invitrogen Canada Inc) to the reaction mixture. Subsequently, the DNA was transformed into NM522 cells where the uracil-containing templates were degraded. Plasmid DNA recovered from the transformants was further transformed into JM109, recovered from this strain and used to screen for the desired mutation in the plasmid sub-clone by DNA sequencing. 


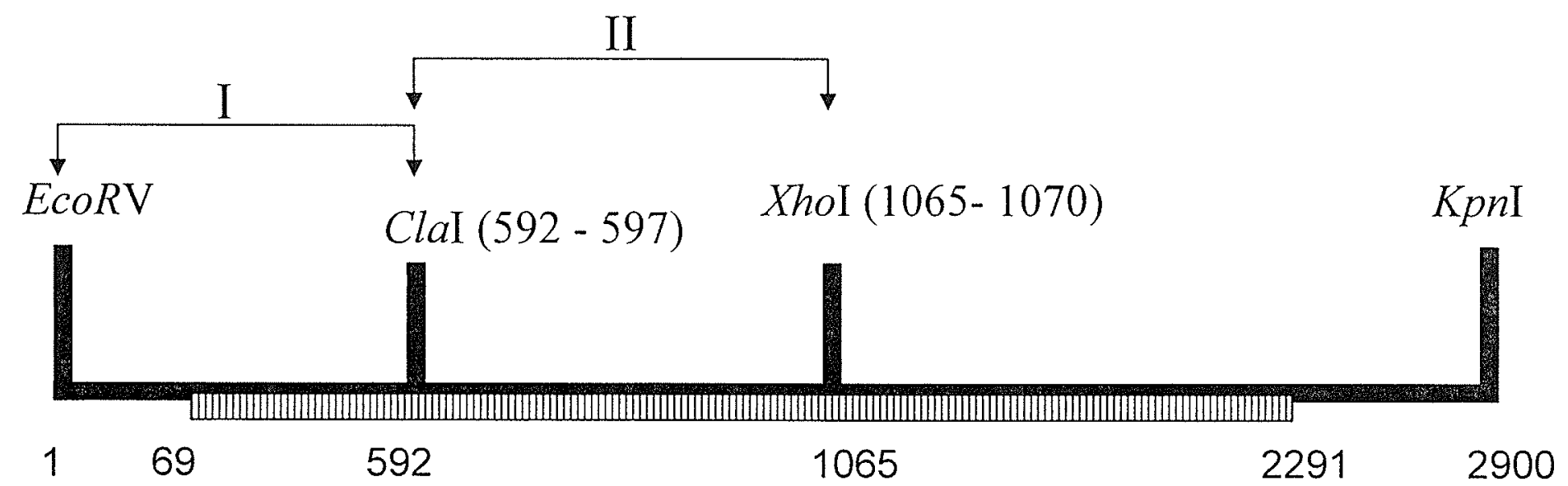

Figure 2.1. Simplified restriction map of the chromosomal insert containing the $M$. tuberculosis kat $G$ gene in plasmids

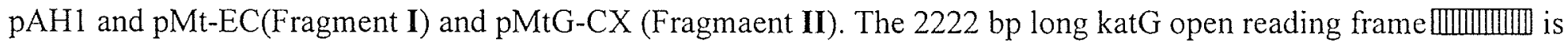
indicated as the part of the chromosomal insert as is the insert as are the two insert fragments I and II used to construct the subclone vectors employed in site directed mutagenesis of $M$. tuberculosis kat $G$. 

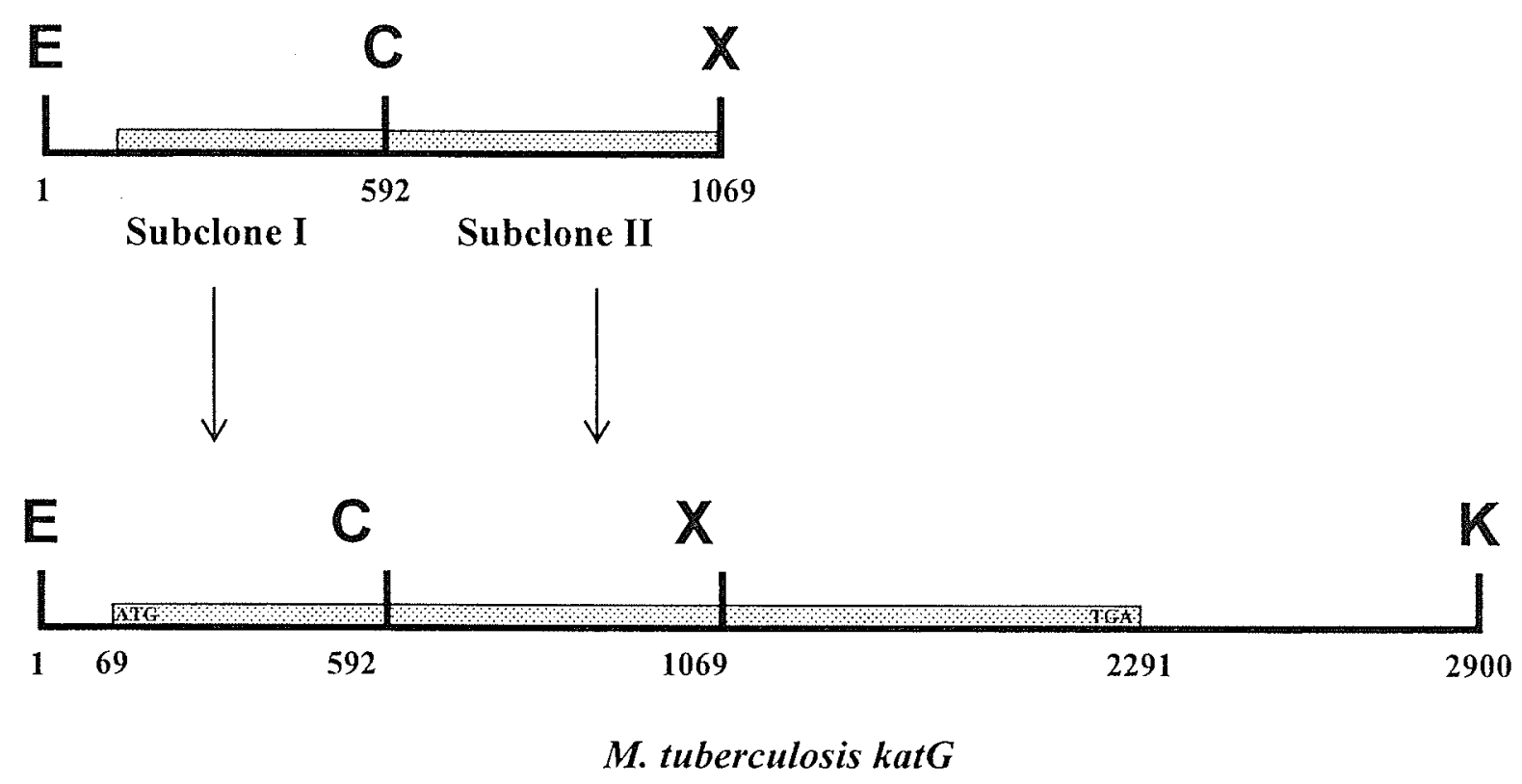

Figure 2.2 General reconstruction protocol used for the generation of variant MtKatG's from mutagenized subclone I for protein expression. (E:EcoRV, K:KpnI, C:ClaI, X:XhoI,) 
Figure 2.3. The DNA sequence and corresponding amino acid sequence of $M$. tuberculosis showing the restriction sites and the target codones selected for mutagenesis in this study. The sequencing primers are also shown.

M. tubercuTosis katG (MtKatG)

GATATCCGACACTTCGCGATCACATCCGTGATCACAGCCCGATAACACCAACTCCTGGAA

60

CTATAGGCTGTGAAGCGCTAGTGTAGGCACTAGTGTCGGGCTATTGTGGTTGAGGACCTT ECORV

BClI

$$
\checkmark \text { P }
$$

GGAATGCTGTGCCCGAGCAACACCCACCCATTACAGAAACCACCACCGGAGCCGCTAGCA

CCTTACGACACGGGCTCGTTGTGGGTGGGTAATGTCTTTGGTGGTGGCCTCGGCGATCGT

$$
\text { NheI }
$$

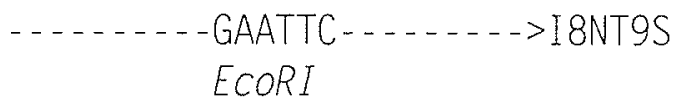

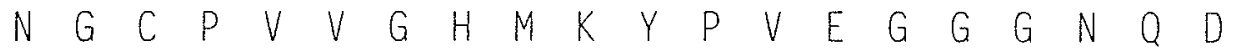
ACGGCTGTCCCGTCGTGGGTCATATGAAATACCCCGTCGAGGGCGGCGGAAACCAGGACT TGCCGACAGGGCAGCACCCAGTATACTTTATGGGGCAGCTCCCGCCGCCTTTGGTCCTGA

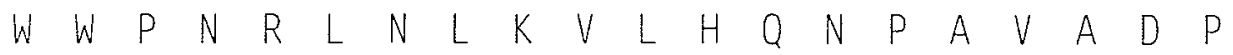
GGTGGCCCAACCGGCTCAATCTGAAGGTACTGCACCAAAACCCGGCCGTCGCTGACCCGA 240 CCACCGGGTTGGCCGAGTTAGACTTCCATGACGTGGTTTTGGGCCGGCAGCGACTGGGCT CCCAACCTGCAGAATCTGA $\rightarrow$ MtyG5

$$
\text { PStI }
$$

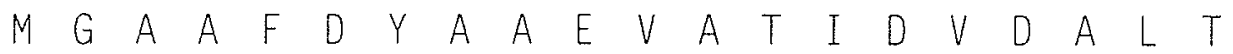
TGGGTGCGGCGTTCGACTATGCCGCGGAGGTCGCGACCATCGACGTTGACGCCCTGACGC ACCCACGCCGCAAGCTGATACGGCGCCTCCAGCGCTGGTAGCTGCAACTGCGGGACTGCG

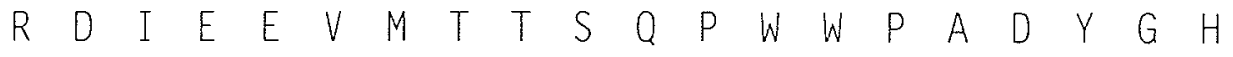
GGGACATCGAGGAAGTGATGACCACCTCGCAGCCGTGGTGGCCCGCCGACTACGGCCACT CCCTGTAGCTCCTTCACTACTGGTGGAGCGTCGGCACCACCGGGCGGCTGATGCCGGTGA -...... - TTC ....... - W91F

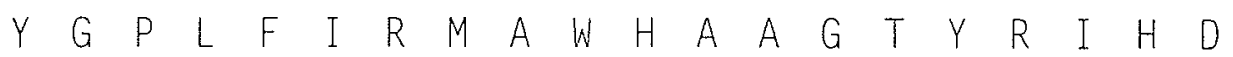
ACGGGCCGCTGTTTATCCGGATGGCGTGGCACGCTGCCGGCACCTACCGCATCCACGACG 420 TGCCCGGCGACAAATAGGCCTACCGCACCGTGCGACGGCCGTGGATGGCGTAGGTGCTGC

G $R \quad G \quad G \quad A \quad G \quad G \quad G \quad M \quad Q \quad R \quad F \quad A \quad P \quad L \quad N \quad S \quad W \quad P \quad D$ (137) GCCGCGGCGGCGCCGGGGGCGGCATGCAGCGGTTCGCGCCGCTTAACAGCTGGCCCGACA CGGCGCCGCCGCGGCCCCCGCCGTACGTCGCCAAGCGCGGCGAATTGTCGACCGGGCTGT

$$
\text { SphI PVUII }
$$


$K \quad L \quad S$ W A $A$ D $L$ L I V V F A G AGCTCTCATGGGCGGACCTGATTGTTTTCGCCGGCAACTGCGCGCTGGAATCGATGGGCT AGTTCTGCAAGCCCAAGCCGAAGCCGGCCCAGCTGGTCACCCTCGGGCTACTCCAGATAA

$$
\text { SalI }<M t K-4
$$

W G K E A $T$ T W L G D E (217) GGGGCAAGGAAGCCACCTGGCTCGGCGATGAGCGTTACAGCGGTAAGCGGGATCTGGAGA CCCCGTTCCTTCGGTGGACCGAGCCGCTACTCGCAATGTCGCCATTCGCCCTAGACCTCT NCOI Sa $\quad$ II

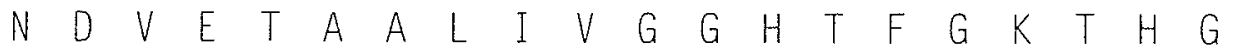
(277) ACGACGTCGAAACAGCGGCGCTGATCGTCGGCGGTCACACTTTCGGTAAGACCCATGGCG 900 TGCTGCAGCTTTGTCGCCGCGACTAGCAGCCGCCAGTGTGAAAGCCATTCTGGGTACCGC NCOI CCGGCCCGGCCGATCTGGTCGGCCCCGAACCCGAGGCTGCTCCGCTGGAGCAGATGGGCT (297) GGCCGGGCCGGCTAGACCAGCCGGGGCTTGGGCTCCGACGAGGCGACCTCGTCTACCCGA

960

$L G \quad W \quad K \quad S \quad S \quad Y \quad G \quad T \quad G \quad T \quad G \quad K \quad D \quad A \quad I \quad T \quad S \quad G \quad I$ TGGGCTGGAAGAGCTCGTATGGCACCGGAACCGGTAAGGACGCGATCACCAGCGGCATCG ACCCGACCTTCTCGAGCATACCGTGGCCTTGGCCATTCCTGCGCTAGTGGTCGCCGTAGC 
$\ldots>S 315 T$

MtK6

$-\ldots>S 315 N$

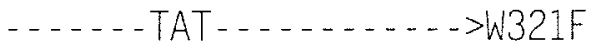

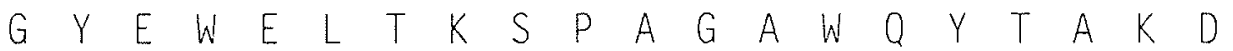
GCTACGAGTGGGAGCTGACGAAGAGCCCTGCTGGCGCTTGGCAATACACCGCCAAGGACG GCGCCGGTGCCGGCACCATCCCGGACCCGTTCGGCGGGCCAGGGCGCTCCCCGACGATGC CGCGGCCACGGCCGTGGTAGGGCCTGGGCAAGCCGCCCGGTCCCGCGAGGGGCTGCTACG TGGCCACTGACCTCTCGCTGCGGGTGGATCCGATCTATGAGCGGATCACGCGTCGCTGGC ACCGGTGACTGGAGAGCGACGCCCACCTAGGCTAGATACTCGCCTAGTGCGCAGCGACCG BamHI AfIIII(MTUI)

$L$ E H P E E L A D E $F$ A TGGAACACCCCGAGGAATTGGCCGACGAGTTCGCCAAGGCCTGGTACAAGCTGATCCACC 1320 ACCTTGTGGGGCTCCTTAACCGGCTGCTCAAGCGGTTCCGGACCATGTTCGACTAGGTGG

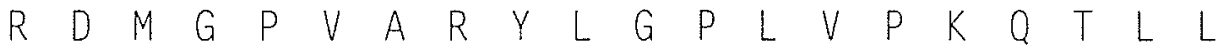
GAGACATGGGTCCCGTTGCGAGATACCTTGGGCCGCTGGTCCCCAAGCAGACCCTGCTGT 1380

CTCTGTACCCAGGGCAACGCTCTATGGAACCCGGCGACCAGGGGTTCGTCTGGGACGACA ....... - GAATTC . . . . . . PMPtKatGI

ECORI

GGTCCCGTCGACAGATACCT>pMtkatG4

Sal I

MtyG7<GCAACGCTTCGAAGAACCC HindIII

W Q D P V P A V S H D L V G E A E I A S (457) GGCAGGATCCGGTCCCTGCGGTCAGCCACGACCTCGTCGGCGAAGCCGAGATTGCCAGCC 1440 CCGTCCTAGGCCAGGGACGCCAGTCGGTGCTGGAGCAGCCGCTTCGGCTCTAACGGTCGG BamHI 
$\begin{array}{llllllllllllllllllll} & K & S & Q & I & R & A & S & G & L & T & V & S & Q & L & V & S & T & A & W\end{array}$ TTAAGAGCCAGATCCGGGCATCGGGATTGACTGTCTCACAGCTAGTTTCGACCGCATGGG AATTCTCGGTCTAGGCCCGTAGCCCTAACTGACAGAGTGTCGATCAAAGCTGGCGTACCC AfTII

$\begin{array}{llllllllllllllllllllll}\text { A A A } & S & S & F & R & G & S & D & K & R & G & G & A & N & G & G & R & I & & \text { (497) }\end{array}$ CGGCGGCGTCGTCGTTCCGTGGTAGCGACAAGCGCGGCGGCGCCAACGGTGGTCGCATCC 1560 GCCGCCGCAGCAGCAAGGCACCATCGCTGTTCGCGCCGCCGCGGTTGCCACCAGCGTAGG

$$
\text { ...................... MtK7 }
$$

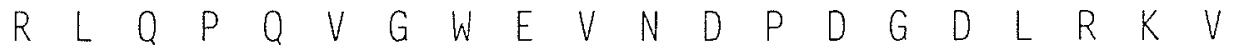
GCCTGCAGCCACAAGTCGGGTGGGAGGTCAACGACCCCGACGGGGATCTGCGCAAGGTCA CGGACGTCGGTGTTCAGCCCACCCTCCAGTTGCTGGGGCTGCCCCTAGACGCGTTCCAGT

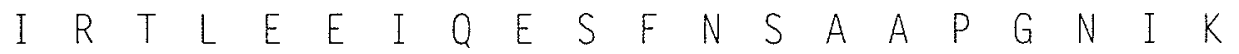
TTCGCACCCTGGAAGAGATCCAGGAGTCATTCAACTCCGCGGCGCCGGGGAACATCAAAG AAGCGTGGGACCTTCTCTAGGTCCTCAGTAAGTTGAGGCGCCGCGGCCCCTTGTAGTTTC

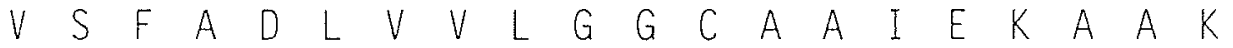
TGTCCTTCGCCGACCTCGTCGTGCTCGGTGGCTGTGCCGCCATAGAGAAAGCAGCAAAGG ACAGGAAGCGGCTGGAGCAGCACGAGCCACCGACACGGCGGTATCTCTTTCGTCGTTTCC

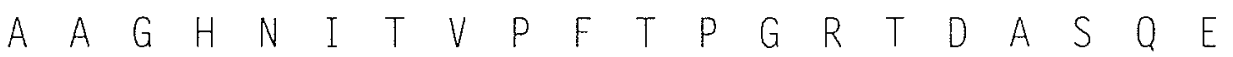
CGGCTGGCCACAACATCACGGTGCCCTTCACCCCGGGCCGCACGGATGCGTCGCAGGAAC GCCGACCGGTGTTGTAGTGCCACGGGAAGTGGGGCCCGGCGTGCCTACGCAGCGTCCTTG Sma I

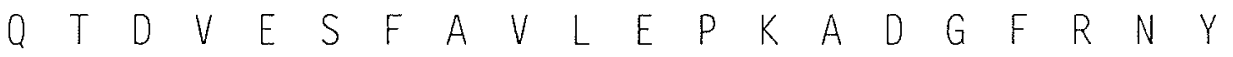
AAACCGACGTGGAATCCTTTGCCGTGCTGGAGCCCAAGGCAGATGGCTTCCGAAACTACC TTTGGCTGCACCTTAGGAAACGGCACGACCTCGGGTTCCGTCTACCGAAGGCTTTGATGG

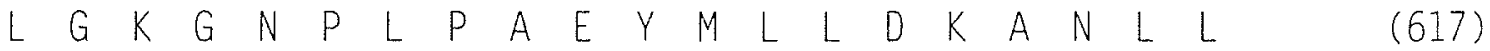
TCGGAAAGGGCAACCCGTTGCCGGCCGAGTACATGCTGCTCGACAAGGCGAACCTGCTTA 1920 AGCCTTTCCCGTTGGGCAACGGCCGGCTCATGTACGACGAGCTGTTCCGCTTGGACGAAT 
$T$ T S A P CGCTCAGTGCCCCTGAGATGACGGTGCTGGTAGGTGGCCTGCGCGTCCTCGGCGCAAACT 1980 GCGAGTCACGGGGACTCTACTGCCACGACCATCCACCGGACGCGCAGGAGCCGCGTTTGA $\begin{array}{llllllllllllllllllllll}Y & K & R & L & P & L & G & V & F & T & E & A & S & E & S & L & T & N & D & F & & \text { (657) }\end{array}$ ACAAGCGCTTACCGCTGGGCGTGTTCACCGAGGCCTCCGAGTCACTGACCAACGACTTCT 2040 TGTTCGCGAATGGCGACCCGCACAAGTGGCTCCGGAGGCTCAGTGACTGGTTGCTCAAGA ECO47III

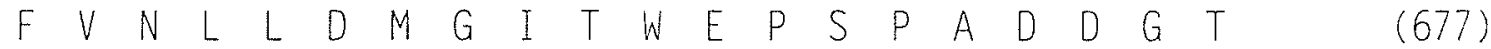
TCGTGAACCTGCTCGACATGGGTATCACCTGGGAGCCCTCGCCAGCAGATGACGGGACCT 2100 AGCACTTGGACGAGCTGTACCCATAGTGGACCCTCGGGAGCGGTCGTCTACTGCCCTGGA

$\begin{array}{llllllllllllllllllllll}Y & 0 & G & K & D & G & S & G & K & V & K & W & T & G & S & R & V & D & L & V & & \text { (697) }\end{array}$ ACCAGGGCAAGGATGGCAGTGGCAAGGTGAAGTGGACCGGCAGCCGCGTGGACCTGGTCT 2160 TGGTCCCGTTCCTACCGTCACCGTTCCACTTCACCTGGCCGTCGGCGCACCTGGACCAGA

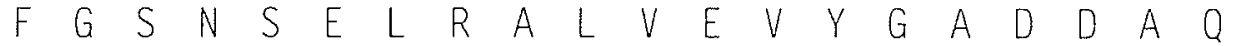
TCGGGTCCAACTCGGAGTTGCGGGCGCTTGTCGAGGTCTATGGCGCCGATGACGCGCAGC 2220 AGCCCAGGTTGAGCCTCAACGCCCGCGAACAGCTCCAGATACCGCGGCTACTGCGCGTCG

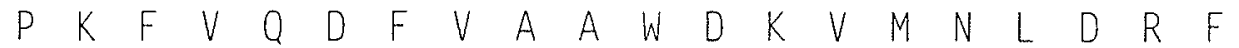
CGAAGTTCGTGCAGGACTTCGTCGCTGCCTGGGACAAGGTGATGAACCTCGACAGGTTCG (737) GCTTCAAGCACGTCCTGAAGCAGCGACGGACCCTGTTCCACTACTTGGAGCTGTCCAAGC 2280 PMtKatG3-CTGTCCAAGT

D V R * ACGTGCGCTGATTCGGGTTGATCGGCCCTGCCCGCCGATCAACCACAACCCGCCGCAGCA TGCACGCGACTAAGCCCAACTAGCCGGGACGGGCGGCTAGTTGGTGTTGGGCGGCGTCGT

-..... - TTCGAA - ... - pMtKatG2

$$
-M t K-2
$$

HindIII

TCGAACGCTGAT

Hind I I (................. CCATGGCCATGG $<$ MTG $^{\prime} \mathrm{Kpn}$

CCCCGCGAGCTGACCGGCTCGCGGGGTGCTGGTGTTTGCCCGGCGCGATTTGTCAGACCC 2400 GGGGCGCTCGACTGGCCGAGCGCCCCACGACCACAAACGGGCCGCGCTAAACAGTCTGGG CGCGTGCATGGTGGTCGCACGGACGCACGAGACGGGGATGACGAGACGGGGATGAGGAGA GCGCACGTACCACCAGCGTGCCTGCGTGCTCTGCCCCTACTGCTCTGCCCCTACTCCTCT TTCCCGCGGCTTTACACGACCTACACGCTAGTGGGCCTTCGGTGGCGGCTCCTCATGGAG 
GACGAGGTGTACGGGATAATGCTCATGCATGGCTGGGCGGTACAGCACGTGGAGTGCGAG 2580 CTGCTCCACATGCCCTATTACGAGTACGTACCGACCCGCCATGTCGTGCACCTCACGCTC Nsi I

CGACGGCCATTTGCCTACACGGTTGGTCTAACCCGGCGCGGCTTGCCCGAACTGGTGGTG 2640 GCTGCCGGTAAACGGATGTGCCAACCAGATTGGGCCGCGCCGAACGGGCTTGACCACCAC ACTGGCCTCTCGCCACGACGTGGGCAGCGGTTGTTGAACATGCCGTCGAGGGCTCTGGTC 2700 TGACCGGAGAGCGGTGCTGCACCCGTCGCCAACAACTTGTACGGCAGCTCCCGAGACCAG GGTGACTTGCTGACTCCCGGTATGTAGACCACCCTCAAAGCCGGCCCTCTTGTCGAAACG 2760 CCACTGAACGACTGAGGGCCATACATCTGGTGGGAGTTTCGGCCGGGAGAACAGCTTTGO

GTCCAGGCTACACATCCGGACGCGCATTTGTATTGTGCGATCGCCATCTTTGCGCACAAG 2820 CAGGTCCGATGTGTAGGCCTGCGCGTAAACATAACACGCTAGCGGTAGAAACGCGTGTTC GTGACGGCCTTGCAGTTGGTGTGGGCCGACCGCGTGGTCGCTGGCCGTGGGCGGCGGACT 2880 CACTGCCGGAACGTCAACCACACCCGGCTGGCGCACCAGCGACCGGCACCCGCCGCCTGA TCGACGAAGGTCGCGGTACC 2900 AGCTGCTTCCAGCGCCATGG KpnI 


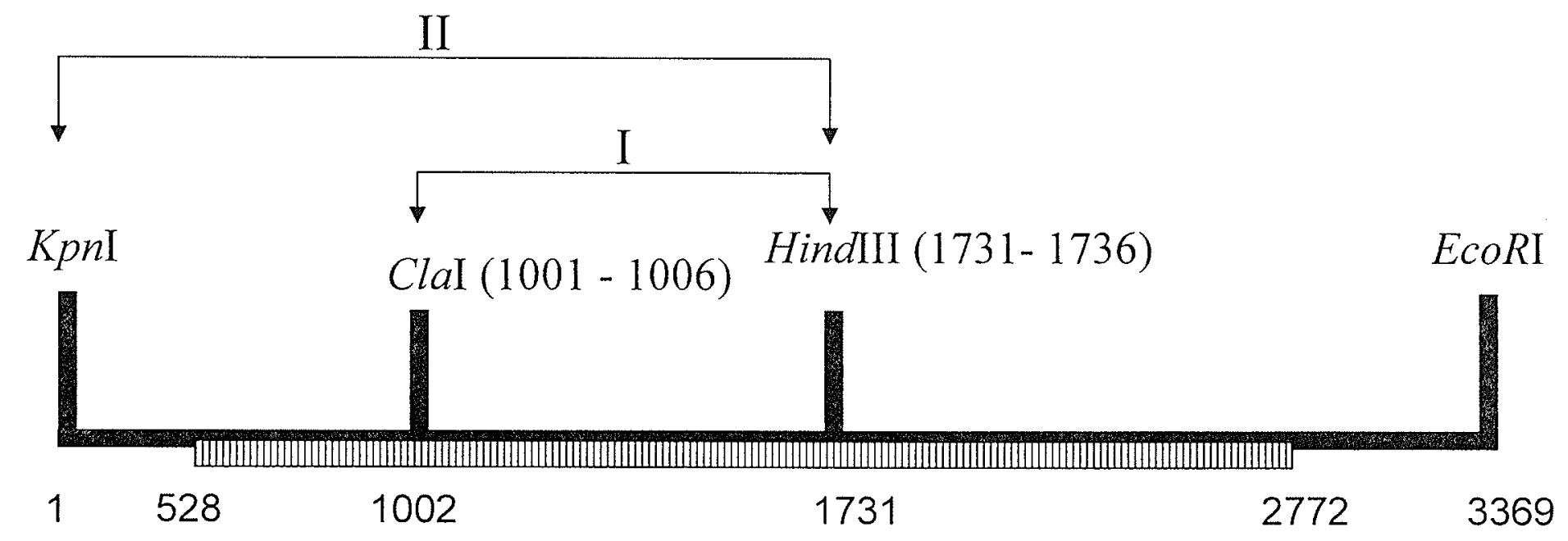

Figure 2.4. Simplified restriction map of the chromosomal insert containing the B. pseudomallei $k a t G$ gene in plasmids pBpG and pBpG-KH (Fragment I) and pBpG-CH (Fragmaent II). The 1134 bp long katG open reading frame indicated as the part of the chromosomal insert as is the insert fragment I used to construct the subclone vectors employed in site directed mutagenesis of B. pseudomallei katG. 


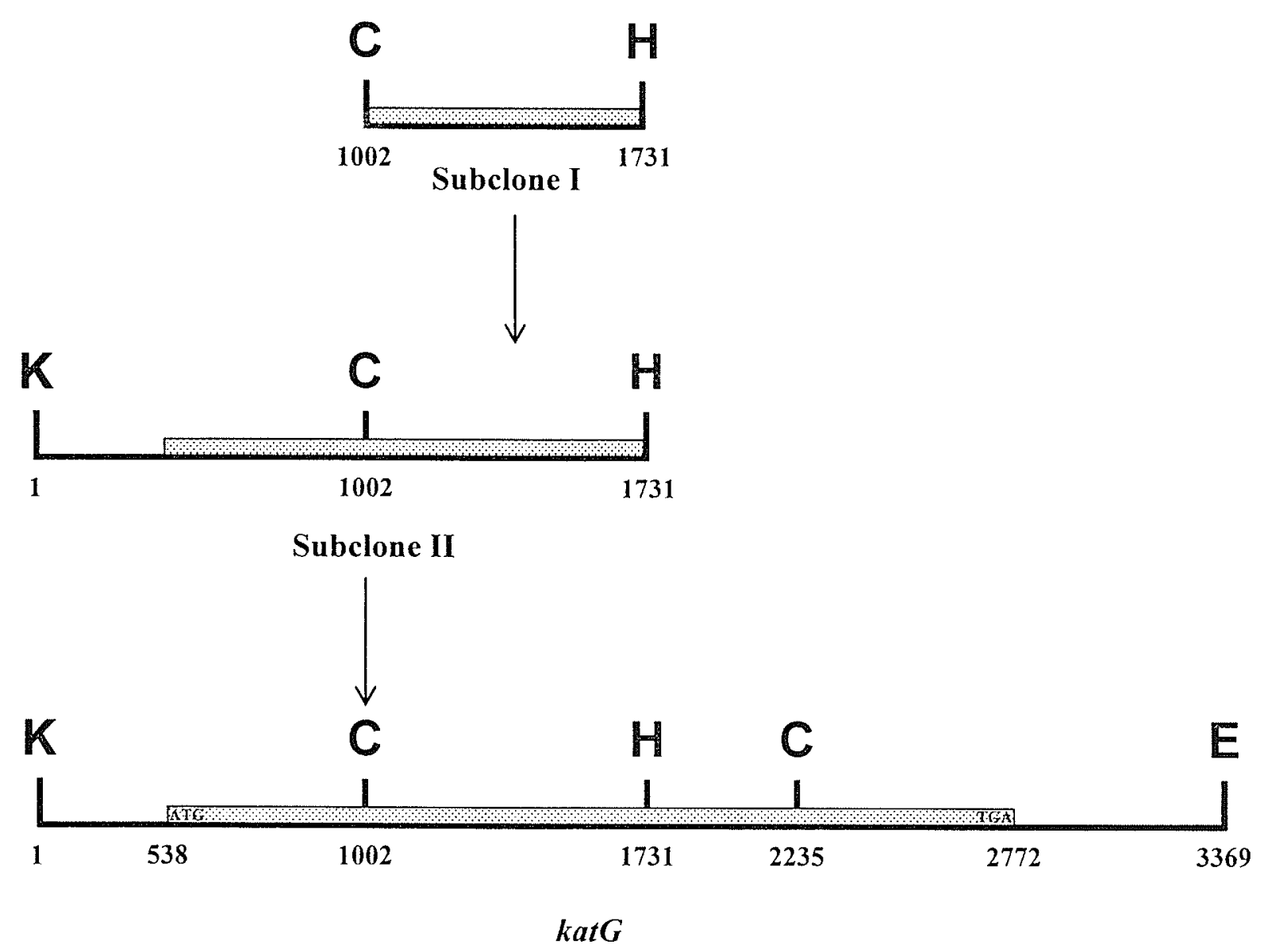

Figure 2.5 General reconstruction protocol used for the generation of variant BpKatG's from mutagenized subclone I for protein expression. (K:KpnI, C:ClaI, HindIII, E: EcoRI) 
Figure 2.6. The DNA sequence and corresponding amino acid sequence of $B$. pseudomallei showing the restriction sites and the target codones selected for mutagenesis in this study. The sequencing primers are also shown.

Burkholderia pseudoma7lej KatG (BpKAtG)

CGGGGTGACGAAGCTCGATATCCCGGCGACGGTGAATTGAGCGCGGCGAGCGCAGCTG 60 GCCCCACTGCTTCGAGCTATAGGGCCGCTGCCACTTAACTCGCGCCGCTCGCGACGAC

CGGGCCGCCGCGCGCCATGATCGGACGGGGCTTCGGGGCCCCGTTTATTTTTGCCTATCG 120 GCCCGGCGGCGCGCGGTACTAGCCTGCCCCGAAGCCCCGGGGCAAATAAAAACGGATAGC

GATAAATAAAATTTATTAAATTACATATATCAATAGCAAATAATAGAATGCTTCGCATGG 180 CTATTTATTTTAAATAATTTAATGTATATAGTTATCGTTTATTATCTTACGAAGCGTACC

ATCGGCGCAGCGCCGCCGGACGGTAACTGCAAGCGTCAAGGGAGGATGTCATGATGCAAC 240 TAGCCGCGTCGCGGCGGCCTGCCATTGACGTTCGCAGTTCCCTCCTACAGTACTACGTTG CGGCATTGTCGCGCGCGCGGCGGAACATTGCGCCGCGCCCGGCGGTCATTGATCGTGCCA 300 GCCGTAACAGCGCGCGCGCCGCCTTGTAACGCGGCGCGGGCCGCCAGTAACTAGCACGGT AGGCTGCCATCCGGAGCCTGCGTCCGATTGCGTTCGCTTATCTGGCGGACCGCGCCCACG TCCGACGGTAGGCCACGGACGCAGGCTAACGCAAGCGAATAGACCGCCTGGCGCGGGTGC GCGGCTGAGCGCCGCCGCGTGCCGGGCCGCTTTTTTCGCGCCGCGCGTCGCGTCGCACGC CGCCGACTCGCGGCGGCGCACGGCCCGGCGAAAAAAGCGCGGCGCGCAGCGCAGCGTGCG

AGGAGGGGTCAACCTGTTTGTTTCCCCGCAAAGTCGCCCCCCGAACGATGCCCGGCTCCG 480 TCCTCCCCAGTTGGACAAACAAAGGGGCGTTTCAGCGGGGGGCTTGCTACGGGCCGAGGC -.................

-....... $>$ BpG5

$\begin{array}{llllllllllllllllllll}G & A & G & P & R & R & R & G & V & H & E & Q & R & R & N & R & M & S & N & E\end{array}$ ATGCCGGGCCCCGCAGGCGGGGCGTACACGAACAAAGGAGAAATCGCATGTCGAATGAAG TACGGCCCGGGGCGTCCGCCCCGCATGTGCTTGTTTCCTCTTTAGCGTACAGCTTACTTC CGAAGTGCCCGTTCCATCAAGCCGCAGGCAACGGCACGTCGAACCGGGACTGGTGGCCCA GCTTCACGGGCAAGGTAGTTCGGCGTCCGTTGCCGTGCAGCTTGGCCCTGACCACCGGGT 
N Q L L D D L ATCAGCTGGACCTGAGCATCCTGCATCGGCACTCGTCGCTGTCCGATCCGATGGGCAAGG

TAGTCGACCTGGACTCGTAGGACGTAGCCGTGAGCAGCGACAGGCTAGGCTACCCGTTCC

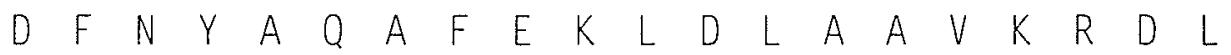
ATTTCAACTACGCGCAGGCGTTCGAGAAGCTCGACCTCGCGGCGGTGAAGCGCGACCTCC TAAAGTTGATGCGCGTCCGCAAGCTCTTCGAGCTGGAGCGCCGCCACTTCGCGCTGGAGG

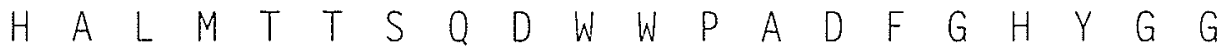
ACGCGCTGATGACGACGTCGCAGGACTGGTGGCCGGCCGATTTCGGCCACTACGGCGGCC (104) TGCGCGACTACTGCTGCAGCGTCCTGACCACCGGCCGGCTAAAGCCGGTGATGCCGCCGG

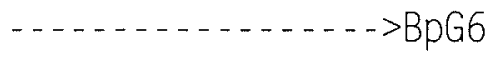

$\begin{array}{llllllllllllllllllll}L & F & I & R & M & A & W & H & S & A & G & T & Y & R & T & A & D & G & R & G\end{array}$ TGTTCATCCGCATGGCATGGCACAGCGCGGGCACGTACCGCACGGCCGACGGCCGCGGCG ACAAGTAGGCGTACCGTACCGTGTCGCGCCCGTGCATGGCGTGCCGGCTGCCGGCGCCGC

$G \quad A \quad G \quad E \quad G \quad Q \quad Q \quad R \quad F \quad A \quad P \quad L \quad N \quad S \quad W \quad P \quad D \quad N \quad A \quad N$ GCGCGGGCGAAGGGCAGCAGCGCTTCGCGCCGCTCAACAGCTGGCCCGACAACGCGAACC CGCGCCCGCTTCCCGTCGTCGCGAAGCGCGGCGAGTTGTCGACCGGGCTGTTGCGCTTGG

$\begin{array}{llllllllllllllllllll}L & D & K & A & R & R & L & L & W & P & I & K & Q & K & Y & G & R & A & I & S\end{array}$ TCGACAAGGCGCGCCGGCTGCTGTGGCCGATCAAGCAGAAGTACGGCCGCGCCATCTCGT AGCTGTTCCGCGCGGCCGACGACACCGGCTAGTTCGTCTTCATGCCGGCGCGGTAGAGCA

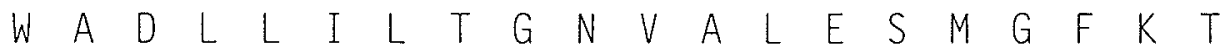
GGGCCGACCTGCTGATCCTGACGGGCAACGTCGCGCTCGAATCGATGGGCTTCAAGACCT CCCGGCTGGACGACTAGGACTGCCCGTTGCAGCGCGAGCTTAGCTACCCGAAGTTCTGGA ClaI

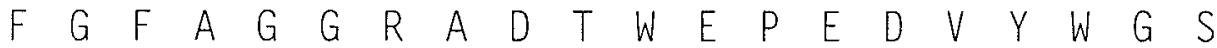
TCGGCTTCGCGGGCGGCCGCGCGGACACGTGGGAGCCCGAGGACGTCTACTGGGGCTCGG AGCCGAAGCGCCCGCCGGCGCGCCTGTGCACCCTTCGGGCTCCTGCAGATGACCCCGAGC

840 900

960 1020

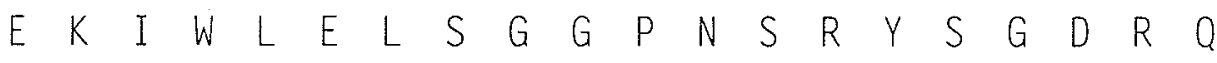
AAAAGATCTGGCTGGAACTGAGCGGCGGCCCGAACAGCCGCTATTCGGGCGACCGCCAGC CTTTTCTAGACCGACCTTGACTCGCCGCCGGCTTGTCGGCGATAAGCCCGCTGGCGGTCG

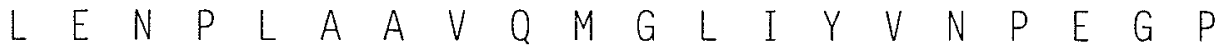
TCGAGAACCCGCTCGCCGCCGTGCAGATGGGCCTCATCTACGTGAATCCGGAAGGCCCGG AGCTCTTGGGCGAGCGGCGGCACGTCTACCCGGAGTAGATGCACTTAGGCCTTCCGGGCC 1200

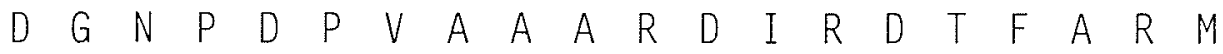


ACGGCAATCCCGATCCGGTCGCCGCGGCGCGCGACATTCGTGACACCTTCGCGCGCATGG 1260 TGCCGTTAGGGCTAGGCCAGCGGCGCCGCGCGCTGTAAGCACTGTGGAAGCGCGCGTACC

A M N D E E E $T$ T $V$ A $A$ L I A (284) CGATGAACGACGAAGAGACGGTCGCGCTGATCGCGGGCGGCCACACGTTCGGCAAGACGC 1320 GCTACTTGCTGCTTCTCTGCCAGCGCGACTAGCGCCCGCCGGTGTGCAAGCCGTTCTGCG -...>BPG3

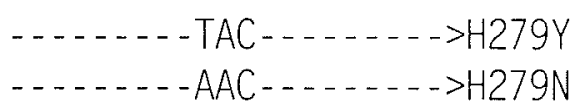

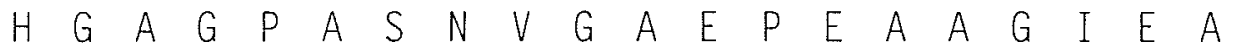
ACGGCGCGGGGCCCGCGTCGAACGTCGGCGCCGAGCCGGAGGCCGCGGGCATCGAAGCGC TGCCGCGCCCCGGGCGCAGCTTGCAGCCGCGGCTCGGCCTCCGGCGCCCGTAGCTTCGCG

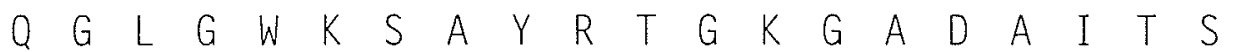
AGGGCCTCGGCTGGAAGAGCGCGTACCGCACGGGCAAGGGCGCGGACGCGATCACGAGCG TCCCGGAGCCGACCTTCTCGCGCATGGCGTGCCCGTTTCCCGCGCCTGCGCTAGTGCTCGC

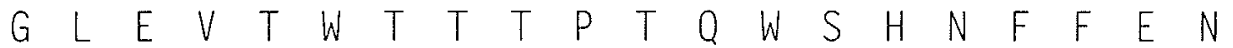
GGCTCGAAGTCACGTGGACGACGACGCCGACGCAGTGGAGCCACAACTTCTTCGAGAACC CGGAGCTTCAGTGCACCTGCTGCTGCGGCTGCGTCACCTCGGTGTTGAAGAAGCTCTTGG

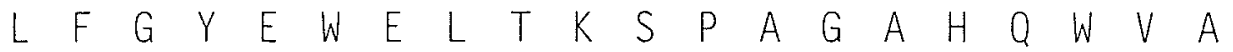
TGTTCGGCTACGAGTGGGAGCTGACGAAGAGCCCGGCGGGCGCGCACCAGTGGGTCGCGA ACAAGCCGATGCTCACCCTCGACTGCTTCTCGGGCCGCCCGCGCGTGGTCACCCAGCGCT

$\begin{array}{llllllllllllllllllll}K & G & A & D & A & V & I & P & D & A & F & D & P & S & K & K & H & R & P & T\end{array}$ AGGGCGCCGACGCGGTGATTCCCGACGCGTTCGATCCGTCGAAGAAGCATCGTCCGACGA TCCCGCGGCTGCGCCACTAAGGGCTGCGCAAGCTAGGCAGCTTCTTCGTAGCAGGCTGCT

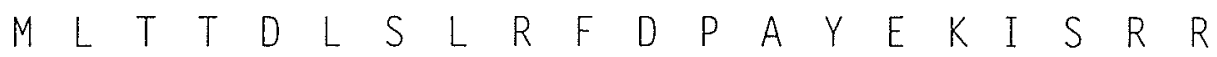
TGCTCACGACCGACCTGTCGCTGCGCTTCGATCCGGCGTACGAAAAGATCTCGCGCCGCT ACGAGTGCTGGCTGGACAGCGACGCGAAGCTAGGCCGCATGCTTTTCTAGAGCGCGGCGA

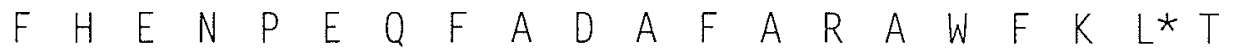
TCCACGAGAACCCGGAGCAGTTCGCCGACGCGTTCGCGCGCGCCTGGTTCAAGCTTACGC AGGTGCTCTTGGGCCTCGTCAAGCGGCTGCGCAAGCGCGCGCGGACCAAGTTCGAGTGCG

H R D M G P R A R Y ACCGCGACATGGGCCCGCGCGCGCGCTATCTCGGCCCGGAAGTGCCGGCCGAGGTGCTGC TGGCGCTGTACCCGGGCGCGCGCGCGATAGAGCCGGGCCTTCACGGCCGGCTCCACGACG - >BPGI (324) (364)

(304)

1380

1440

1500

1560

1620

(404)

1680

1740

1800 


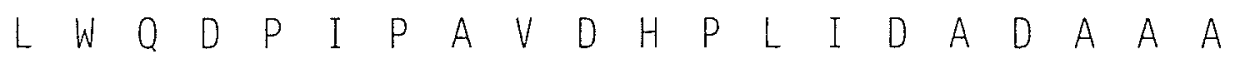
(464) TGTGGCAGGACCCGATTCCGGCCGTCGACCATCCGCTGATCGACGCCGCGGACGCCGCCG ACACCGTCCTGGGCTAAGGCCGGCAGCTGGTAGGCGACTAGCTGCGGCGCCTGCGGCGGC

E $L \quad K \quad A \quad K \quad V \quad L \quad A \quad S \quad G \quad L \quad T \quad V \quad S \quad Q \quad L \quad V \quad S \quad T A$ AGCTGAAGGCAAAGGTGCTCGCGTCGGGGCTGACCGTGTCGCAGCTCGTTTCCACCGCGT 1920 TCGACTTCCGTTTCCACGAGCGCAGCCCCGACTGGCACAGCGTCGAGCAAAGGTGGCGCA

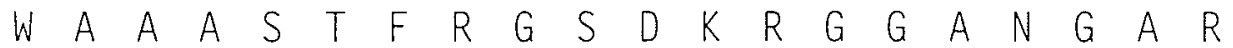
GGGCGGCGGCGTCCACCTTCCGCGGCTCGGACAAGCGCGGCGGCGCGAACGGCGCGCGCA CCCGCCGCCGCAGGTGGAAGGCGCCGAGCCTGTTCGCGCCGCCGCGCTTGCCGCGCGCGT

$\begin{array}{llllllllllllllllllll}I & R & L & A & P & Q & K & D & W & E & A & N & Q & P & E & Q & L & A & A & Y\end{array}$ TTCGCCTTGCGCCGCAGAAGGACTGGGAGGCGAACCAGCCCGAGCAGCTCGCGGCGGTGC AAGCGGAACGCGGCGTCTTCCTGACCCTCCGCTTGGTCGGGCTCGTCGAGCGCCGCCACG

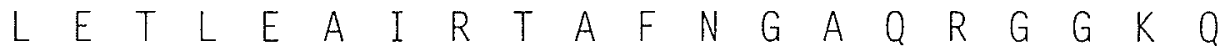
(544) TCGAGACGCTCGAGGCAATTCGCACGGCGTTCAACGGCGCGCAGCGCGGCGGCAAGCAAG 2100 AGCTCTGCGAGCTCCGTTAAGCGTGCCGCAAGTTGCCGCGCGTCGCGCCGCCGTTCGTTC

$V$ S L A D D $L$ L I V V L A $G$ G C A G (564) TGTCGCTCGCCGATCTGATCGTGCTGGCCGGCTGCGCGGGCGTCGAGCAGGCGGCGAAGA 2160 ACAGCGAGCGGCTAGACTAGCACGACCGGCCGACGCGCCCGGAGCTCGTCCGCCGCTTCT

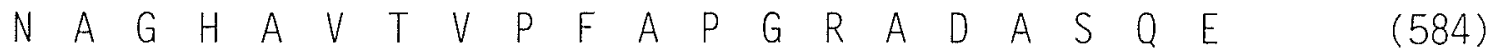
ACGCGGGCCACGCGGTGACCGTGCCGTTCGCGCCGGGCCGCGCGGACGCATCGCAGGAGC 2220 TGCGCCCGGTGCGCCACTGGCACGGCAAGCGCGGCCCGGCGCGCCTGCGTAGCGTCCTCG

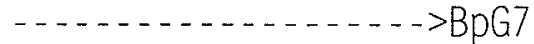

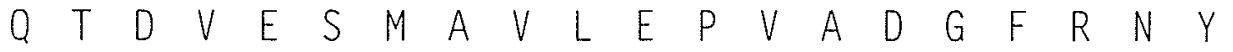
(604) AGACCGACGTCGAATCGATGGCCGTGCTCGAGCCGGTGGCCGACGGTTTTCGCAACTACC 2280 TCTGGCTGCAGCTTAGCTACCGGCACGAGCTCGGCCACCGGCTGCCAAAAGCGTTGATGG ClaI

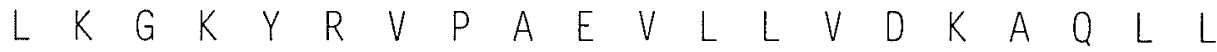
(624) TGAAGGGCAAGTATCGGGTGCCCGCCGAGGTGCTGCTCGTCGACAAGGCGCAACTGCTGA 2340 ACTTCCCGTTCATAGCCCACGGGCGGCTCCACGACGAGCAGCTGTTCCGCGTTGACGACT

$T \quad L \quad S \quad A \quad P \quad E \quad M \quad T \quad V \quad L \quad L \quad G \quad G \quad L \quad R \quad V \quad L \quad G \quad A \quad N$ CGCTGAGCGCGCCGGAGATGACGGTGCTGCTGGGCGGCCTGCGCGTGCTGGGCGCGAACG GCGACTCGCGCGGCCTCTACTGCCACGACGACCCGGCGGACGCGCACGACCCGCGCTTGC 
$\begin{array}{llllllllllllllllllllll}V & G & O & S & R & H & G & V & F & T & A & R & E & \text { Q } & A & L & T & N & D & F & & \text { (664) }\end{array}$ TCGGGCAGAGCCGGCACGGCGTGTTCACCGCGCGCGAGCAGGCATTGACCAACGACTTCT 2460 AGCCCGTCTCGGCCGTGCCGCACAAGTGGCGCGCGCTCGTCCGTAACTGGTTGCTGAAGA

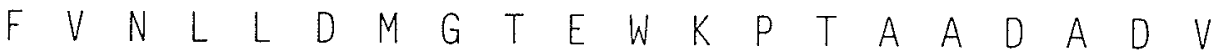
(684) TCGTGAACCTGCTCGACATGGGCACCGAGTGGAAGCCGACGGCGGCCGACGCGGACGTGT AGCACTTGGACGAGCTGTACCCGTGGCTCACCTTCGGCTGCCGCCGGCTGCGCCTGCACA

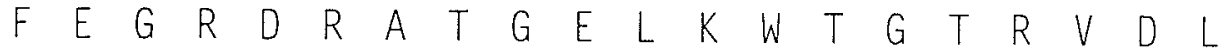
TCGAAGGGCGCGACCGCGCGACGGGCGAGCTCAAGTGGACGGGCACGCGCGTCGATCTCG AGCTTCCCGCGCTGGCGCGCTGCCCGCTCGAGTTCACCTGCCCGTGCGCGCAGCTAGAGC

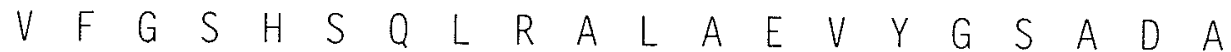
TGTTCGGCTCGCACTCGCAGTTGCGCGCGCTCGCGGAGGTCTACGGCAGCGCGGACGCGC ACAAGCCGAGCGTGAGCGTCAACGCGCGCGAGCGCCTCCAGATGCCGTCGCGCCTGCGCG

Q E K F V R D F V A V V W N K V V M N $N$ L D R AGGAGAAGTTCGTGCGCGACTTCGTCGCGGTCTGGAACAAGGTGATGAACCTCGACCGCT TCCTCTTCAAGCACGCGCTGAAGCAGCGCCAGACCTTGTTCCACTACTTGGAGCTGGCGA

$F \quad D \quad L \quad A *$

TCGATCTCGCGTGATCGCGCCGCCGCGCCGCCGGAGCGGCGGCGCGCGGCGGCGGGGAAC AGCTAGAGCGCACTAGCGCGGCGGCGCGGCGGCCTCGCCGCCGCGCGCCGCCGCCCCTTG BpG4<..............

GGCCGGCTGACGCGGGCCGCTTCCCGCCGGGCCGCTGATATCGTTTCAAGGAGTGACGAT CCGGCCGACTGCGCCCGGCGAAGGGCGGCCCGGCGACTATAGCAAAGTTCCTCACTGCTA BpG6< ................ . .

CATGACGCAAATGATTCTCGACCTGCGCGGGGCGCTCGCGGCGCCGGCCAGGCGCGCGGG CGCCGCGGCGTGGCGGCGGCTGATCGGCCTCGCGATCGCGGGCGGCGGCGCGGCCGTGCT GGCCGCGCAGGCCGCGCGCTACTTCACGGCGGCCGCCGGCGGTTGAGCGGGCCTGCGTTT CGCGCAAATCGCGCGCTCGCGATCTACGCTAAACTGGTGCGGCGCTCGGCGGGCAGCCGC ACGCGCGTCTGCCGCCTCTGCATAGGCTGCCCATGCGCATGTCGCCTGCGCGCATCCCGC ATCGGGCATGCGGATCTTTCGATGCATTTTTCGTCGGTTCGAACCATCGGACAAGGAGTTT CGAGGATGGCCAAGAAAAGCAACGCAACCCAGATCAACATCGGCATCAGCGACAAGGATC GCAAGAAGATCGCGGCGGGGCTGTCGCGTCTGCTCGCCGATACGTACACGCTGTACCTGA AGACGCACAATTTCCACTGGAACGTGACCGGCCCGATGTTCAACACGCTGCACCTGATGT TCGAGGAGCAGTACAACGAACTGTGGCTCGCCGTCGATCTCGTCGCGGAGCGCATCCGCA CGCTCGGGGTCGTCGCGCCGGGCACGTATCGCGAATTCGCGAAGCTGTCGTCGATTCCCG AGGCCGACGGCGTGCCGGCCGCCGAGGAGATGATTCGCCAGCTCGTCGAAGGGCATGAGG CTGTCGTGCGCACCGCGCGCGCGATCTTCCCGGACGCCGACGCGGCGAGCGACGAGCCCA CCGCCGATCTGCTGACGCAGCGCCTGCAG

(704)

2580 2640 2700 2760

2820

2880

2940

3000

3060

3120

3180

3240

3300

3360

3420

3480

3540

3600

3629 
Once the correct mutation was identified, the complete sub-clone sequence was determined to ensure that no base changes apart from those desired had been introduced. The mutated sub-clone was used to reconstruct the complete $M t k a t G$ or Bpkat $G$ gene. The complete gene was sequenced over the region containing the mutation for final confirmation. Reconstructed mutant $M t k a t G$ and Bpkat $G$ clones were transformed into UM262 for determination of enzyme activities and visualization of protein from crude extracts or whole cells by SDS-PAGE. Clones over expressing significant levels of variant MtKatG and BpKatG enzyme were grown on a large scale (4-6 liters) for purification and characterization of the enzymes.

\subsubsection{Reconstruction of $M$. tuberculosis kat $G$ subclones with desired mutation}

An outline of the reconstruction protocol is illustrated in Figure 2.2a. The plasmids was drawn in a linearized form for reasons of simplicity. Reconstruction of mutant $k a t G$ genes was done in a single step. Plasmid pMt-CX (subclone I) with the desired mutation was cut with XhoI and ClaI. The $474 \mathrm{bp}$ XhoI-ClaI fragment from pMtCX was then ligated into pAH1 that was also cut with $X$ hoI and ClaI to generate the plasmid containing the mutant $k a t G$ gene. All the variant constructs were verified for correct restriction sites using various restriction digestion studies at each step in the construction process.

\subsubsection{Reconstruction of $B$. pseudomallei katG subclones with desired mutation}

An outline of the reconstruction protocol is illustrated in Figure 2.2b. The plasmids have been drawn in a linearized form for reasons of simplicity. Reconstruction 
of mutant $k a t G$ genes was done in two stages. In the first stage, plasmid pBpG-CH (subclone I) with the desired mutation was cut with ClaI and HindIII. The 730 bp ClaIHindIII fragment from $\mathrm{pBpG-CH}$ was then ligated into pBpG-KH (subclone II) that was also cut with ClaI and HindIII. In the second stage, plasmid pBpG-KH (subclone II) with the desired mutation was cut with KpnI and HindIII. The 1731 bp KpnI-HindIII fragment from $\mathrm{pBpG}-\mathrm{KH}$ (subclone II) was then ligated into $\mathrm{pBpG}$ that was also cut with KpnI and HindIII to generate the plasmid containing mutant $k a t G$ gene. All the variant constructs were verified for correct restriction sites using various restriction digestion studies at each step in the construction process.

\subsubsection{DNA isolation and purification}

Isolation of plasmid DNA was according to Sambrook et al. (1989). Plasmid harboring cells were grown in tubes containing $5 \mathrm{~mL} \mathrm{LB}$ medium to stationary phase and were pelleted by centrifugation and resuspended in $200 \mu \mathrm{L}$ Tris-glucose-EDTA buffer (25 mM Tris-HCL, pH 8.0, 1\% glucose, 10 mM Na-EDTA). Resuspended cells were lysed by addition of $400 \mu \mathrm{L} 1 \% \mathrm{SDS}(\mathrm{w} / \mathrm{v}), 0.2 \mathrm{M} \mathrm{NaOH}$ solution and gentle mixing. This was then neutralized by addition of $300 \mu \mathrm{L} 6.2 \mathrm{M}$ ammonium acetate, pH 6.2. After 10 min incubation, the mixture was centrifuged twice to remove the precipitate. Plasmid were pelleted by centrifugation and resuspended in $200 \mu \mathrm{L}$ Tris-glucose-EDTA buffer (25 mM Tris-HCL, pH 8.0, 1\% glucose, 10 mM Na-EDTA). Resuspended cells were lysed by addition of $400 \mu \mathrm{L} 1 \%$ SDS (w/v), $0.2 \mathrm{M} \mathrm{NaOH}$ solution and gentle mixing. This was then neutralized by addition of $300 \mu \mathrm{L} 6.2 \mathrm{M}$ ammonium acetate, pH 6.2. After 10 min incubation, the mixture was centrifuged twice to remove the precipitate. Plasmid 
DNA was then precipitated by the addition of $550 \mu \mathrm{L}$ isopropanol to the remaining supernatant followed by 15 min incubation at room temperature. Plasmid DNA was pelleted by centrifugation, washed twice with $1 \mathrm{~mL}$ of $70 \%(\mathrm{v} / \mathrm{v})$ ice cold ethanol, and then dried under vacuum. The DNA pellet was either stored under these conditions at $20^{\circ} \mathrm{C}$ or was resuspended in HPLC grade distilled water or TE buffer $(10 \mathrm{mM}$ Tris, $\mathrm{pH}$ 8.0, $1 \mathrm{mM} \mathrm{Na}$-EDTA) prior to being stored at $-20^{\circ} \mathrm{C}$ until further use.

\subsubsection{Restriction endonuclease digestion of DNA}

Restriction digestions were performed at $37^{\circ} \mathrm{C}$ for $2-5 \mathrm{~h}$ in a total volume of 10 $\mu \mathrm{L}$ containing $1 \mu \mathrm{L}$ RNAse, $1 \mu \mathrm{L}$ of $10 \mathrm{X}$ appropriate buffer provided by the supplier, $~$ 1-5 $\mu \mathrm{g}$ DNA, and 0.5-1 $\mu \mathrm{l}$ (50-2500 Units) of endonuclease.

\subsubsection{Agarose gel electrophoresis}

Electrophoresis of the restriction endonuclease digested DNA were performed according to Sambrook et al., (1989). Agarose gels containing 1\%(w/v) agarose and 0.9 $\mu \mathrm{g} / \mathrm{mL}$ ethidium bromide were prepared in TAE buffer ( $40 \mathrm{mM}$ Tris-Acetate and $1 \mathrm{mM}$ EDTA, pH 8.0) and cast in Bio-Rad Mini Sub cell plexiglass horizontal electrophoresis trays $(6.5 \mathrm{~cm} \times 10 \mathrm{~cm})$. Samples of $10 \mu \mathrm{l}$ volumes were mixed with $2 \mu \mathrm{l}$ stop buffer $(40 \%$ [v/v] glycerol, $10 \mathrm{mM}$ EDTA pH 8.0, 0.25\% [w/v] bromophenol blue). $1 \mathrm{~kb}$ or $1 \mathrm{~kb}$ plus DNA ladders (Invitrogen Canada Inc) were used as molecular weight size standards. Electrophoresis was carried out at 40-60 mA constant current in TAE buffer until the bromophenol blue marker dye front had migrated approximately two thirds of the length of the gel. Following electrophoresis, the DNA bands were visualized with ultraviolet 
light and recorded in a digitized form using a Gel Doc 1000 image capture system (BioRad) or FluorChem ${ }^{\mathrm{TM}} 8900$ image capture system (Alpha Innotech).

\subsubsection{Ligation}

DNA fragments to be ligated were excised from agarose gels and purified using the Ultraclean ${ }^{\mathrm{TM}} 15$ DNA purification kit (Bio/Can Scientific Inc.) according to the instructions supplied by the manufacturer. Ligation of insert DNA into vectors was carried out according to the procedure of Sambrook et al (1989). Purified DNA was mixed in a ratio of 2-3 of insert to vector in $10 \mu \mathrm{l}$ volumes containing 1 unit of T4 DNA ligase (Invitrogen Canada Inc.), and the manufacturer's supplied buffer at the appropriate concentration. Ligation mixtures were incubated overnight at $15^{\circ} \mathrm{C}$. A mixture without added insert DNA was used as control.

\subsubsection{Transformation}

Transformation of $E$. coli cells with the various plasmids was achieved according to Chung et al (1989). $5 \mathrm{~mL}$ LB cultures of cells grown to exponential phase $(2-4 \mathrm{~h})$ were harvested by centrifugation and made competent by resuspension in $500 \mu \mathrm{L}$ ice-cold 0.1 $\mathrm{M} \mathrm{CaCl}_{2}$ for at least $30 \mathrm{~min}$ on ice. 2-10 mg DNA was added to $100 \mu \mathrm{L}$ of this cell suspension, followed by additional 30 min incubation on ice, and a 90 second heat shock at $42^{\circ} \mathrm{C} .0 .9 \mathrm{~mL} \mathrm{LB}$ was added to the cell suspension and incubated at $37^{\circ} \mathrm{C}$ for one hour without aeration. The mixture was either spread, or (in the case of ligation mixtures transformations) mixed with $3 \mathrm{~mL}$ of molten $\left(50^{\circ} \mathrm{C}\right) \mathrm{R}$-Top agar $(0.125 \mathrm{~g}$ of yeast extract, $1.25 \mathrm{~g}$ of tryptone, $1 \mathrm{~g}$ of $\mathrm{NaCl}, 1 \mathrm{~g}$ of agar per $125 \mathrm{~mL}$ volume with $0.25 \mathrm{~mL}$ of $1 \mathrm{M}$ 
$\mathrm{CaCl}_{2}$ and $0.42 \mathrm{~mL}$ of $30 \%$ glucose sterile solutions added after autoclaving) and poured onto ampicillin-containing LB plates.

\subsubsection{DNA sequencing}

Sequencing of DNA was performed according to the method of Sanger et al. (1977). Sequencing was carried out manually with double stranded DNA templates using the primers shown in Table 2.2. For preparation of double stranded DNA template, $5 \mu \mathrm{g}$ plasmid DNA was resuspended and denatured in a $40 \mu \mathrm{L}$ volume of freshly prepared $2 \mathrm{M}$ $\mathrm{NaOH}$. This mixture was incubated for $10 \mathrm{~min}$ at $37^{\circ} \mathrm{C}$, reprecipated by addition of $10 \mu \mathrm{L}$ of $3 \mathrm{M}$ sodium acetate (pH 4.8) and $140 \mu \mathrm{L}$ of ice-cold $95 \%$ ethanol. Following incubation at $-20^{\circ} \mathrm{C}$ for $30 \mathrm{~min}$, the DNA pellet was recovered by centrifugation, washed once with $1 \mathrm{~mL}$ of $95 \%$ ice-cold ethanol, and once with $200 \mu \mathrm{L}$ of $70 \%$ ice-cold ethanol, and then evaporated to dryness under vacuum in a desiccator. Annealing and sequencing reactions were carried out using a $10 \mu \mathrm{Ci}\left[\alpha_{-}{ }^{35} \mathrm{~S}\right] \mathrm{dATP}$ (Amersham biosciences). Reaction mixtures were separated and resolved on $8 \%(\mathrm{w} / \mathrm{v})$ polyacrylamide vertical slab gels containing $7 \mathrm{M}$ urea, $0.13 \mathrm{M}$ Tris, $0.13 \mathrm{M}$ boric acid, and $10 \mathrm{mM}$ EDTA. Electrophoresis was carried out using 18-24 mA constant current in TBE buffer $(90 \mathrm{mM}$ Tris, $89 \mathrm{mM}$ borate, $2.2 \mathrm{mM}$ EDTA) for $1.5-4 \mathrm{~h}$ as required. Gels were mounted on $3 \mathrm{~mm}$ paper (Whatman), covered with clear plastic film, and dried at $80^{\circ} \mathrm{C}$ for about 1 hour on a slab gel drier vacuum (Savant). Dried gels were exposed to X-ray film (Kodak X-OMAT $A R)$ in order to visualize and record the DNA bands. 


\subsection{Purification of catalase-peroxidases}

For small scale crude extracts used to determine relative levels of protein expression and catalase activity, plasmid-containing cells were grown in either $5 \mathrm{~mL}$ of LB medium in test tubes, or in $30 \mathrm{~mL}$ of LB medium in shaking flasks at $28^{\circ} \mathrm{C}$ and $37^{\circ} \mathrm{C}$ for $16-20 \mathrm{~h}$. The cells were pelleted and resuspended in 1-2 $\mathrm{mL}$ of $50 \mathrm{mM}$ potassium phosphate buffer, $\mathrm{pH} 7.0$ (buffer A), sonically disrupted, and centrifuged to remove unbroken cells and debris. This was followed by an assay for catalase activity and a protein expression profile by electrophoresis on sodium dodecyl sulfate polyacrylamide gels (SDS-PAGE). If only protein visualization was to be carried out (in the case of mutations that had low or no catalase activity), 10-15 $\mu$ l aliquots of the cell suspensions were pelleted by centrifugation, directly resuspended in SDS protein sample buffer, and analyzed via SDS-PAGE.

For large scale preparations of KatG and their variant proteins, UM262 cells over expressing the desired protein from the appropriate plasmid borne genes were grown in 4-6 liters of LB media, in 2 L shake flasks (500 mL LB per flask) supplemented with 100 $\mu \mathrm{g} / \mathrm{mL}$ of ampicillin and $40 \mu \mathrm{g} / \mathrm{mL}$ hemin (Sigma), for $16-20 \mathrm{~h}$, at either $28^{\circ} \mathrm{C}$ or $37^{\circ} \mathrm{C}$ with good aeration. Isolation and purification of KatGs were done according to the procedure of Loewen and Switala (1986) with modifications. All isolation and purification steps were carried out at $4^{\circ} \mathrm{C}$. Cells were harvested from the growth medium by centrifugation at $7000 \mathrm{rpm}$. The cell pellet (approximately $30 \mathrm{~g}$ wet weight from $6 \mathrm{l}$ culture) was either stored at $-60^{\circ} \mathrm{C}$ or resuspended in about $150-250 \mathrm{~mL}$ of buffer $\mathrm{A}$ containing $5 \mathrm{mM}$ EDTA. The cells were disrupted by a single pass through a French pressure cell press at 20,000 psi. Unbroken cells and debris were removed by 
centrifugation, yielding the crude extract, which was treated with streptomycin sulfate to a final concentration of $2.5 \%(\mathrm{w} / \mathrm{v})$. In case of protein preparations from $A$. fulgiduis $\mathrm{KatG}$ and $B$. stereothermopilus $\mathrm{KatG}$, the crude extract was heat treated at $80^{\circ} \mathrm{C}$ in a water bath for $45 \mathrm{~min}$ followed by centrifugation prior to streptomycin sulfate treatment. The resulting precipitants were removed by centrifugation and discarded. Solid $\left(\mathrm{NH}_{4}\right)_{2} \mathrm{SO}_{4}$ was added in appropriate amounts with gentle stirring, to precipitate the desired protein. Most of the $\mathrm{KatG}$ proteins were found to precipitate in $\left(\mathrm{NH}_{4}\right)_{2} \mathrm{SO}_{4}$ at 40 $50 \%$ saturation. Pellets from the $\left(\mathrm{NH}_{4}\right)_{2} \mathrm{SO}_{4}$ precipitations were resuspended in $10-20 \mathrm{~mL}$ of buffer A. The presence of the desired protein in the pellets was confirmed by assays for catalytic activity and visualization on SDS gels. Resuspensions were centrifuged to remove any remaining precipitate, and dialyzed overnight against 2 liters of buffer $\mathrm{A}$ using a 12,000-14,000 molecular weight cutoff membrane,

The dialyzed resuspensions were centrifuged and loaded onto a $2.5 \mathrm{~cm} \times 23 \mathrm{~cm}$ column of DEAE-cellulose A-500 (Cellufine, Amicon) equilibrated with buffer A. The column was washed with buffer $A$ until the $A_{280}$ of the eluting solution was below 0.04 . The protein was then eluted with a $0-0.4 \mathrm{M} \mathrm{NaCl}$ linear gradient in buffer $\mathrm{A}$, usually to a volume of $1 \mathrm{~L}$. Eighty drops $(5 \mathrm{~mL})$ fractions of the eluted proteins from the column were collected throughout. The purity of the recovered column fractions was determined using $A_{280}, A_{407}$ and catalase activity and the fractions with higher $A_{280}, A_{407}$ and higher catalase activity were selected. Selected fractions were pooled and concentrated under nitrogen in a stirred pressure cell (Model 8050, Amicon) using a YM-30 (Amicon) membrane, to volumes between 5-10 mL. The concentrated protein sample was dialyzed against approximately $1 \mathrm{~L}$ of buffer $\mathrm{A}$. The concentrated, dialyzed proteins were checked 
for purity using catalase activity, the $\mathrm{Rz}$ (Reinheitzahl) values as determined by $\mathrm{A}_{407 / 280}$ ratios and visualization using SDS-PAGE. The protein was loaded on to a $2.5 \times 15 \mathrm{~cm}$ hydroxyapatite column (Bio-Rad) equilibrated with potassium phosphate $5 \mathrm{mM}(\mathrm{pH} 7.0)$. The protein was eluted with potassium phosphate $5-200 \mathrm{mM}, \mathrm{pH} 7.0$ usually in a final volume of $500 \mathrm{~mL}$. Fifty drop fractions of the eluted proteins from the column were collected through out. Selected fractions were pooled and concentrated as previously. The purified samples were aliquoted into centrifuge tubes in $0.5 \mathrm{~mL}$ volumes and stored frozen at $-60^{\circ} \mathrm{C}$ until use.

\subsection{Polyacrylamide gel electrophoresis (PAGE) of proteins and staining}

Denaturing SDS-PAGE was carried out according to Weber et al (1972).

Discontinuous $4 \%$ stacking and $8 \%$ separating polyacrylamide gels were cast as vertical slabs of dimensions $10 \times 10 \mathrm{~cm}$ and $0.5 \mathrm{~mm}$ thickness (mini gels). Samples loaded onto the mini gels usually contained about $10-20 \mu \mathrm{g}$ protein for crude extracts or $2-5 \mu \mathrm{g}$ protein for purified protein. Protein samples were mixed with equal volumes of reducing and denaturing sample buffer $\left(3.4 \mathrm{mg} / \mathrm{mL} \mathrm{NaH}_{2} \mathrm{PO} 4,10.2 \mathrm{mg} / \mathrm{mL} \mathrm{Na}_{2} \mathrm{HPO}_{4}, 10 \mathrm{mg} / \mathrm{mL}\right.$ SDS, $0.13 \mathrm{mM} 2$-mercaptoethanol, $0.36 \mathrm{~g} / \mathrm{mL}$ urea and $0.15 \%$ bromophenol blue) and boiled for $3 \mathrm{~min}$ before loading on the gels. Samples were run with $150 \mathrm{~V}$ constant voltage in a vertical BIO-RAD Mini Protean II electrophoresis system, using a running buffer containing $14 \mathrm{~g}$ glycine, $3 \mathrm{~g}$ Tris base and $1 \mathrm{~g}$ SDS per L. Gels were stained in a solution containing $0.5 \mathrm{~g} / \mathrm{l}$ Coomassie Brilliant Blue R-250, 30\% ethanol and $10 \%$ acetic acid for one hour and destained with repeated changes of destaining solution containing $15 \%$ methanol and $7 \%$ acetic acid and $1 \%$ glycerol for $30 \mathrm{~min}$. Gels were then mounted 
on $3 \mathrm{~mm}$ (Whatman) paper, covered with a clear plastic film, and dried at $80^{\circ} \mathrm{C}$ for 1 hour on a slab gel drier under vacuum (Savant).

Non-denaturing PAGE was carried out according to Davis (1964). 8\% acrylamide continuous gels were cast as vertical slabs as described for SDS-PAGE. When protein was to be visualized on the gels, sample amounts loaded were as described for SDSPAGE. When enzymatic activity was to be visualized on the gels, samples were loaded in amounts corresponding to 1 unit of catalase activity, or 40 units of peroxidase activity. Samples were mixed with equal volumes of $2 \mathrm{X}$ sample buffer containing $0.125 \mathrm{M}$ Tris, pH 6.8, 10\% (w/v) glycerol, and $0.2 \%$ bromophenol blue (modified from Bio-Rad MiniProtean II instruction manual). Electrophoresis was performed as described for SDSPAGE, but using a running buffer containing $4.8 \mathrm{~g}$ Tris base, $20.8 \mathrm{~g}$ glycine per $4 \mathrm{~L}$.

Gels were stained for peroxidase activity according to the method of Gregory and Fridovich (1974) using 3,3'-diaminobenzidine (DAB) as the electron donor species. Gels were soaked in a solution containing $20 \mathrm{mg}$ DAB in $80 \mathrm{~mL}$ of $50 \mathrm{mM}$ potassium phosphate buffer, $\mathrm{pH} 7.0$ for $45 \mathrm{~min}$, followed by a brief wash with water and then a second soak in the same buffer containing $20 \mathrm{mM} \mathrm{H}_{2} \mathrm{O}_{2}$. Peroxidase activity was visualized as brown band on the gels. Colour development was usually complete within $30 \mathrm{~min}$.

Gels were stained for catalase activity according to the method of Clare et al. (1984) with minor modifications. Gels were soaked in a solution containing $50 \mu \mathrm{g} / \mathrm{mL}$ horseradish peroxidase (Sigma) in $50 \mathrm{mM}$ potassium phosophate, $\mathrm{pH} 7.0$ for $45 \mathrm{~min}$, then briefly washed with water, incubated in $20 \mathrm{mM} \mathrm{H}_{2} \mathrm{O}_{2}$ for $10 \mathrm{~min}$, and lastly, soaked in a solution of $50 \mathrm{mg} / \mathrm{mL} \mathrm{DAB}$ in $80 \mathrm{~mL}$ water containing $1 \mathrm{~mL}$ glycerol. Catalase activity 
was visualized as zones of clearing on brown background. Color development was usually complete within 30-60 min.

Staining for KatG-mediated oxidation of isoniazid (INH) was performed by soaking the gels in $200 \mathrm{~mL}$ Tris-HCl, pH 8.0 containing $10 \mathrm{mM} \mathrm{NNH}$ (Sigma), and 0.2 mM Nitro Blue tetrazolium (NBT). INH oxidation was visualized as purple bands on the gels. Color development was usually complete between 30-60 min. When staining of gels was judged to be complete, they were rinsed with water, and soaked in $7 \%$ acetic acid, $1 \%$ glycerol for several h or overnight prior to mounting.

Staining for KatG mediated NADH oxidation was performed by soaking the gels in $200 \mathrm{~mL} 50 \mathrm{mM}$ Tris- $\mathrm{HCl}, \mathrm{pH} 8.75$ containing $200 \mu \mathrm{M}$ NADH and $0.2 \mathrm{mM} \mathrm{NBT}$. $\mathrm{NADH}$ oxidation was visualized as purple bands on the gels. Color development was complete in 1-2 h. When staining of gels was judged to be complete, they were rinsed with water, and soaked in $7 \%$ acetic acid, $1 \%$ glycerol for several h or overnight prior to mounting.

\subsection{Enzyme assay and protein quantitation}

\subsubsection{Catalase activity}

Calalase activity was determined by the method of Rorth and Jensen (1967) in a Gilson oxygraph equipped with a Clark electrode. One unit of catalase is defined as the amount of enzyme that decomposes $1 \mu \mathrm{M} \mathrm{H}_{2} \mathrm{O}_{2}$ in $1 \mathrm{~min}$ in $60 \mathrm{mM} \mathrm{H}_{2} \mathrm{O}_{2}$ at $37^{\circ} \mathrm{C}$, pH 7.0. Appropriately diluted samples of enzyme or cell cultures aliquots were incubated in 1.8 $\mathrm{mL} 50 \mathrm{mM}$ phosphate buffer, $\mathrm{pH} 7.0$ for $0.5-1.0 \mathrm{~min}$ at $37^{\circ} \mathrm{C}$ followed by the addition of $\mathrm{H}_{2} \mathrm{O}_{2}$ to a final concentration of $60 \mathrm{mM}$. Catalase activity as units $/ \mathrm{mL}$ was determined 
from the slope of the plot representing oxygen evolution. Specific catalase activity was expressed as units/mL per mg purified protein or units per $\mathrm{mg}$ dry cell weight. Specific activities were always determined as the average of a minimum of three or more individual determinations. The data was fitted to Michaelis-Menton equation using Sigma Plot to determine the $\mathrm{V}_{\max }$ and $K_{\mathrm{m}}$ values.

\subsubsection{Peroxidase activity}

Peroxidase activity was determined spectrophotometrically by the $o$-dianisidine method described in the Worthington Enzyme Catalogue (Worthington Chemicals Co., 1969). Assays were carried out at room temperature in $1 \mathrm{~mL}$ final assay volumes containing $1 \mathrm{mM} \mathrm{H}_{2} \mathrm{O}_{2}, 0.34 \mathrm{mM}$ o-dianisidine in $50 \mathrm{mM}$ sodium acetate buffer $\mathrm{pH} 4.5$. Aliquots $(1-5 \mu \mathrm{l})$ of the appropriately diluted enzymes were added to initiate the reaction. Peroxidase activity was determined by the $\Delta \mathrm{A}_{560} / \mathrm{min}$ over periods of $2 \mathrm{~min}$ and expressed as units $\bullet \mathrm{mg}^{-1} \cdot \mathrm{mL}^{-1}$ purified protein calculated as: $(\Delta \mathrm{A} 460 / \mathrm{min}) / 11.3 \mathrm{x} \mathrm{mg}$ enzyme/mL reaction mixture), using a molar extinction coefficient at $\mathrm{A}_{460} \mathrm{~nm}$ for $o$ dianisidine product of $11,300 \mathrm{M}^{-1} \mathrm{~cm}^{-1}$. Peroxidase activity was also determined spectrophotometrically by the $2,2^{2}$-azinobis $\{3$-ethylbenzoathiazolinesulfonic acid $\}$ (ABTS) method (Smith et al., 1990) with minor modifications. Assays were carried out at room temperature in $1 \mathrm{~mL}$ final assay volumes containing $2.5 \mathrm{mM} \mathrm{H}_{2} \mathrm{O}_{2}, 0.4 \mathrm{mM}$ ABTS in $50 \mathrm{mM}$ sodium acetate buffer $\mathrm{pH} 4.5$. Aliquots $(1-5 \mu \mathrm{l})$ of the appropriately diluted enzymes were added to initiate reaction. Peroxidase activity was determined by the $\triangle \mathrm{A}_{405} / \mathrm{min}$ average over a period of $2 \mathrm{~min}$ and expressed as units $\cdot \mathrm{mg}^{-1} \cdot \mathrm{mL}^{-1}$ purified protein calculated as: $\left(\Delta \mathrm{A}_{405} / \mathrm{min}\right) / 36.8 \times \mathrm{mg}$ enzyme $/ \mathrm{mL}$ reaction mixture $)$, using a 
molar extinction coefficient of ABTS product at $A_{405} \mathrm{~nm}$ of $36,800 \mathrm{M}^{-1} \mathrm{~cm}^{-1}$. One unit of peroxidase activity is defined as the amount that decomposes $1 \mu$ mole of electron donor (ABTS or $o$-dianisidine) in 1 minute at $\mathrm{pH} 4.5$ and room temperature.

\subsubsection{INH hydrazine lyase activity}

INH hydrazine lyase activity has been referred to the formation of radical species from a reaction mixture containing INH, KatG and NBT under the conditions described below. The reaction involves removal of the hydrazine moiety from INH (Figure 4.3) resulting in the formation of isonicotinoyl radical which could subsequently form amide, acid and aldehyde derivatives of INH (Nguyen et al., 2001).

Isoniazid (INH) hydrazine lyase by KatG was determined spectrophotometrically at $560 \mathrm{~nm}$ by monitoring the reduction of NBT to a monoformazan dye $\left(\varepsilon=15,000 \mathrm{M}^{-}\right.$ ${ }^{1} \mathrm{~cm}^{-1}$ ) (Auclair and Voicin, 1985) through an enzymatically oxidized radical species of isoniazid. Assays were carried out in $1 \mathrm{~mL}$ final volumes containing $10 \mathrm{mM} \mathrm{INH}$ and 200 $\mu \mathrm{M}$ NBT in $50 \mathrm{mM}$ Tris buffer, $\mathrm{pH} 8.0$, at room temperature. Aliquots of $1.2 \mu \mathrm{M}$ enzymes were added to initiate the reaction. Effect of manganese on INH hydrazine lyase activity was assayed both in the presence and absence of KatG by the addition of $2 \mu \mathrm{M}$ $\mathrm{MnCl}_{2}$ to the reaction mixture carrying $10 \mathrm{mMINH}$ in $50 \mathrm{mM}$ Tris $\mathrm{pH}$ 8.0. The synergistic effect of INH and NADH on NBT reduction was also followed by monoformazan formation (Auclair and Voicin, 1985) by oxidized radical species of INH and NADH. Assays were carried out in $1 \mathrm{~mL}$ final volumes containing $10 \mathrm{mM} \mathrm{INH}, 250$ $\mu \mathrm{g} \mathrm{NADH}$ and $200 \mu \mathrm{M}$ NBT in $50 \mathrm{mM}$ Tris buffer, $\mathrm{pH} 8.0$ at room temperature. Aliquots of $1.2 \mu \mathrm{M}$ enzymes were added to initiate the reaction. 


\subsubsection{NADH oxidation}

$\mathrm{NADH}$ oxidase activity was determined spectrophotometrically at $560 \mathrm{~nm}$ by monitoring the reduction of NBT to a monoformazan dye by enzymatically oxidized radical species of $\mathrm{NADH}$. Assays were carried out in $1 \mathrm{~mL}$ final volumes containing 250 $\mu \mathrm{g} \mathrm{NADH}$ and $200 \mu \mathrm{M}$ NBT in $50 \mathrm{mM}$ Tris buffer, $\mathrm{pH} 8.75$ at room temperature. Aliquots of $1.2 \mu \mathrm{M}$ enzymes were added to initiate the reaction.

$\mathrm{NADH}$ oxidation was directly observed spectrophotometrically at $340 \mathrm{~nm}$ to monitor the rate of NADH disappearance. Assays were carried out in $1 \mathrm{~mL}$ final volumes containing $100 \mu \mathrm{M} \mathrm{NADH}$ in $50 \mathrm{mM}$ Tris buffer, $\mathrm{pH} 8.75$ at room temperature. Aliquots of $1.2 \mu \mathrm{M}$ enzymes were added to initiate the reaction. NADH oxidase activity was determined by the $\Delta_{340} / \mathrm{min}$ average over periods of $15 \mathrm{~min}$. NADH oxidase activities

were expressed as units. $\mathrm{mL}^{-1} \cdot \mathrm{mg}^{-1}$ purified protein, using a molar extinction coefficient of $6,300 \mathrm{M}^{-1} \cdot \mathrm{cm}^{-1}$ for NADH (DeChatelet et al., 1975). One unit of NADH activity is defined as the amount that decomposes $1 \mathrm{nmol}$ of NADH in 1 minute in a solution of 100 $\mu \mathrm{MNADH}$ at $\mathrm{pH} 8.75$ and room temperature. NADH oxidation was also assayed under anaerobic conditions by flushing the reaction mixture, sealed in a glass tube with rubber cork, with nitrogen before initiating the reaction by the addition of the enzyme.

\subsubsection{Isonicotinoyl NAD synthase activity}

Isonicotinoyl NAD synthase activity was measured from a reaction mixture carrying $200 \mu \mathrm{M} I N H, 100 \mu \mathrm{M} \mathrm{NAD}^{+}, 2 \mu \mathrm{M} \mathrm{MnCl}_{2}$ and $1.2 \mu \mathrm{M} \mathrm{KatG}$ in $50 \mathrm{mM}$ Tris $\mathrm{pH}$ 8.0. The adduct formation was monitored at $326 \mathrm{~nm}$ for a period of $30 \mathrm{~min}$ and rates were calculated using the extinction coefficient $\varepsilon=6.9 \mathrm{mM}^{-1} \mathrm{~cm}^{-1}$ (Rawat et al., 2004). 


\subsubsection{Protein Concentration}

Protein concentration $(\mathrm{mg} / \mathrm{mL})$ was estimated spectrophotometrically based on $\mathrm{A}_{280}$ calculated as $\left(\mathrm{A}_{280} \times \mathrm{MW} / \varepsilon\right)$ where $\mathrm{MW}$ is the molecular weight of $\mathrm{Kat} G$ and $\varepsilon$ is the molar extinction coefficient (Lyane, 1957).

\subsection{Absorption spectrophotometry pf KatGs}

Absorption spectra, time courses, and enzyme assays were performed using a Pharmacia Ultrospec 4000 Spectrophotometer, a Milton Roy MR300 Spectrophotometer, Ultrospec 3100 pro or Biochrom Novaspec II visible spectrophotometer (for assaying samples under anaerobic conditions). All experiments were performed at ambient temperature in $1 \mathrm{~mL}$ quartz, semi micro cuvettes. Proteins were diluted in $50 \mathrm{mM}$ potassium phosphate buffer, $\mathrm{pH} 7.0$, unless otherwise stated, and the same buffer was

used as reference. For the peroxidase assays using ABTS and $o$-dianisidine, proteins were diluted in $50 \mathrm{mM}$ sodium acetate buffer, $\mathrm{pH} 4.5$ and the same buffer was used as reference. For NADH oxidation and $\mathrm{INH}$ hydrazine lyase activity assays, $50 \mathrm{mM}$ Tris buffer, pH 8.75 and 8.0 respectively were used and the same buffers were used as reference. For preparation of spectral and time course plots, data collected were transferred to Sigma Plot software.

\subsection{Effects of inhibitors}

The effects of classical heme inhibitors, $\mathrm{KCN}$ and $\mathrm{NaN}_{3}$ on catalase, peroxidase and oxidase activities of $\mathrm{KatG}$ and variants were studied. The enzyme was incubated with 
different concentrations of $\mathrm{KCN}$ and $\mathrm{NaN}_{3}$ for $1 \mathrm{~min}$ in the reaction mixture prior to initiation of the reaction by addition of the substrate.

The effect of superoxide dismutase (SOD) on NADH oxidase activity was assayed by adding $200 \mu \mathrm{g}$ of SOD in the $1 \mathrm{~mL}$ reaction mixture carrying $250 \mu \mathrm{M} \mathrm{NADH}$, $200 \mu \mathrm{M}$ NBT and appropriately diluted enzyme. The assay was performed as described for NADH oxidation.

The effect of pyridoxine and pyridoxal-5-phosphate on NADH oxidase activity was determined by incubating the appropriately diluted enzyme with different concentrations of pyridoxine and pyridoxal-5-phosphate and initiating the reaction by addition of the substrate.

\subsection{High Performance Liquid Chromatography (HPLC) characterization of} reaction products.

Reaction products of $\mathbb{N H}$ hydrazine lyase activity and isonicotinoyl-NAD adduct

formation were characterization using HPLC. For INH hydrazine lyase activity, reaction mixtures carrying $1 \mathrm{mM} \mathrm{INH}$ and $1.2 \mu \mathrm{M} \mathrm{KatG}$ in $50 \mathrm{mM}$ Tris $\mathrm{pH} 8$ were incubated for 3-4 h. Aliquots were kept frozen after the incubation until ready to use.

For NADH oxidation, reaction mixture containing $200 \mu \mathrm{M}$ NADH and $1.2 \mu \mathrm{M}$ KatG in $50 \mathrm{mM}$ Tris pH 8.75 were incubated for $3-4 \mathrm{~h}$ and aliquots were kept frozen at $20^{\circ} \mathrm{C}$ until ready to use.

Isonicotinoyl-NAD synthase activity was characterized using different permutations and combinations of reactants. Adduct formation was analyzed in the presence and absence of $\mathrm{KatG}$ and in the presence and absence of $\mathrm{MnCl}_{2}$ using INH and 
$\mathrm{NADH} / \mathrm{NAD}^{+}$as substrates. In all the reactions, $100 \mu \mathrm{M} \mathrm{NADH} / \mathrm{NAD}^{+}, 200 \mu \mathrm{M} \mathrm{NH}$, 1.2 $\mu \mathrm{M} \mathrm{KatG}$ and $2 \mu \mathrm{M} \mathrm{MnCl} 2$ were used in $50 \mathrm{mM}$ Tris pH 8.0. Samples were incubated for 3-4 $\mathrm{h}$ and aliquots were kept frozen at $-20^{\circ} \mathrm{C}$ until ready to use.

For HPLC analysis samples were centrifuged and transferred into the tubes made $-$ for automated sample injection (Waters Corporation, MA, USA). Samples were loaded on a $4.6 \times 250 \mathrm{~mm}$ column equilibrated with buffer $\mathrm{A}\left(11.7 \mathrm{~g} \mathrm{KH}_{2} \mathrm{PO}_{4}, 2.4 \mathrm{~g} \mathrm{~K}_{2} \mathrm{HPO}_{4}\right.$ and $2.72 \mathrm{~g}$ tert-butyl ammonium hydrogen sulfate in $1 \mathrm{~L} \mathrm{HPLC}$ grade water) packed with Whatman $5 \mu \mathrm{M}$ ODS-3 (C18 coated, Altech HPLC column) and eluted with a gradient of $0-100 \%$ buffer B (buffer $A+$ Methanol to a final concentration of $30 \%$ ) over a period of 52 min and the $100-0 \%$ buffer B from $53-78$ min in a Waters HPLC system with detection at $260 \mathrm{~nm}$. Fractions showing peaks were collected to determine the mass of the reaction products. For preparation of elution profiles, data collected were transferred to Sigma Plot computer software.

\subsection{Mass Spectrometry}

Mass spectrometric analysis was kindly carried out by Dr. LG Donald. KatG was dialyzed into $5 \mathrm{mM}$ ammonium acetate, and $\mathrm{NADH}$ was dialyzed against three changes of $1 \mathrm{~L} 0.1 \mathrm{M}$ ammonium bicarbonate, after which the concentration was determined by $\mathrm{A}_{340}$. The matrix solution was freshly prepared each day at $160 \mathrm{mg} / \mathrm{mL} 2,5-$ dihydroxybenzoic acid in a 3:1 water:acetonitrile, $2 \%$ formic acid solution. A $20-\mu l$ reaction mixture contained $200 \mu \mathrm{g} \mathrm{KatG}, 128 \mu \mathrm{M} \mathrm{NADH}$, and $10 \mathrm{mM} \mathrm{INH}$ in $0.1 \mathrm{M}$ $\mathrm{NH}_{4} \mathrm{HCO}_{3}$ at room temperature. $0.5 \mu \mathrm{l}$ aliquots were removed at $1 \mathrm{~min}, 2 \mathrm{~h}, 4 \mathrm{~h}$, and $18 \mathrm{~h}$ and immediately mixed with $0.5 \mu \mathrm{l}$ of matrix solution on a metal target. Different 
fractions collected from HPLC column were also analyzed in a similar fashion to characterize the reaction products. All samples were analyzed on a quadrupole time of flight (QqTOF) mass spectrometer (Clare et al., 1984). 


\section{RESULTS}

\subsection{Introduction}

The appearance of three crystal structure reports of catalase-peroxidases (KatGs) from Haloarcula marismortui, Burkholderia pseudomallei and Synechococcus PCC 7942 just prior to the beginning of this study, revived interest in these bi-functional heme proteins. The objective of this study was to gain a better understanding of how catalase and peroxidase activities are modulated by the same enzyme, and more importantly their role in the activation of isoniazid ( $\mathrm{NH}$ ). KatGs from seven different organisms including B. pseudomallei (BpKatG), M. tuberculosis (MtKatG), E. coli (EcKatG), Synechocystis PCC6803 (SyKatG), A. fulgidus (AfKatG), B. stereothermophilus (BsKatG), and $R$. capsulatus (RcKatG), each belonging to a different environmental niche, were selected. Plasmid borne $k a t G$ gene from each organism was over expressed in $E$. coli host cells and purified enzyme was compared for various biochemical and biophysical properties such as $\mathrm{pH}$ optimum and enzyme kinetics for catalase and peroxidase activities. Spectral properties and sensitivity to various heme inhibitors, such as cyanide and azide, were also compared for seven KatGs.

Another focus of this study was the role of KatGs in the activation of isoniazid and subsequent formation of isonicotinoyl-NAD adduct. Much of the literature related to the activation of $\mathrm{INH}$ by $\mathrm{KatG}$ has focused on the fate of $\mathrm{INH}$ and possible intermediates involved in the process (Johnsson and Schultz, 1994; Wilming and Johnsson, 1999; Magliozzo and Marcinkeviciene, 1996; Lei et al., 2000). With $\mathrm{NAD}^{+}$included in the mix, the generation of the isonicotinoyl-NAD adduct was observed both with and without KatG present (Wilming and Johnsson, 1999; Magliozzo and Marcinkeviciene, 1996; Lei 
et al., 2000), leading to the suggestions that the role of KatG is limited to the hydrazinolysis of INH and that the subsequent reaction of the isonicotinoyl radical with $\mathrm{NAD}^{+}$is a nonenzymatic event and subsequent formation of isonicotinoyl-NAD adduct is considered as a non-enzymatic event. During this study, pyridine derivatives such as INH, NADH, NADPH, pyridoxine etc were used to analyze their interaction with KatGs and their possible involvement in the formation of isonicotinoyl-NAD.

\subsection{Purification and characterization of BpKatG, MtKatG, EcKatG, SyKatG, BsKatG, AfKatG and RcKatG.}

\subsubsection{Purification of KatGs}

For the over expression of catalase-peroxidase, the $k a t G$ genes from the seven organisms used in this study were first cloned into high expression plasmid vectors and subsequently over-expressed in E.coli host cells (UM262 or BL21). The plasmids used are listed in the Table 2.1. Heme incorporation in the apoprotein has always been a cause of concern in the over expressed $\mathrm{KatG}$ as heme production by the cellular machinery appears insufficient. Over expressed protein often results in lower heme-to-protein ratios, which is also called "reinheitzahl" (Rz) (Dunnford, 1999). To solve this problem, exogenous heme was added to the growth medium to support heme formation and hence incorporation. Table 3.2.1 describes the effect of $\delta$-aminolevulinic acid (ALA), $\mathrm{FeCl}_{3}$ and hemin chloride and temperature on the catalase specific activity of MtKatG. Iron and ALA are precursors in the biosynthesis of heme whereas hemin is a direct source of ferric heme for the apoprotein. Addition of both $\mathrm{ALA}$ and $\mathrm{FeCl}_{3}$ to the growth medium or the 
Table 3.2.1. Effect of $\mathrm{FeCl}_{3}, \delta$-aminolevulinic acid (ALA) and hemin chloride on the catalase activity of MtKatG. E.coli strain UM262 containing plasmids pAH1 (MtKatG) were grown for $16-18 \mathrm{~h}$ at $37^{\circ}$ or $28^{\circ} \mathrm{C}$ in $30 \mathrm{~mL} \mathrm{LB}$ medium supplemented with ampicillin. Activity assays were performed on whole cells and the activity at $37^{\circ} \mathrm{C}$ was considered $100 \%$.

\begin{tabular}{ccccc}
\hline $\begin{array}{c}\text { Growth } \\
\text { temperature } \\
\left({ }^{\circ} \mathrm{C}\right)\end{array}$ & ALA (mM) & $\mathrm{FeCl}_{3}(\mu \mathrm{M})$ & Hemen $(\mu \mathrm{M})$ & $\begin{array}{c}\text { \% Catalase } \\
\text { activity } \\
(\text { units/mg })\end{array}$ \\
\cline { 2 - 4 } $\mathbf{2 8}$ & - & - & - & 40 \\
$\mathbf{3 7}$ & - & - & - & 100 \\
$\mathbf{2 8}$ & 1 & 50 & - & 50 \\
$\mathbf{3 7}$ & 1 & 50 & - & 130 \\
$\mathbf{2 8}$ & - & - & 60 & 40 \\
$\mathbf{3 7}$ & - & - & 60 & 130 \\
\hline
\end{tabular}


Table 3.2.2.a Purification of wild type BpKatG and MtKatG from E. coli strain UM262 harboring plasmid encoded kat $G$ of $B$. pseudomallei and $M$. tuberculosis.

\begin{tabular}{|c|c|c|c|c|c|}
\hline Purification Step & $\begin{array}{l}\text { Total } \\
\text { protein } \\
\text { (mg) }\end{array}$ & $\begin{array}{l}\text { Total catalase } \\
\text { activity (units } \\
\quad \times 10^{3} \text { ) }\end{array}$ & $\begin{array}{l}\text { Specific } \\
\text { catalase } \\
\text { activity } \\
\text { (units/mg) }\end{array}$ & $\begin{array}{l}\text { Recovery } \\
(\%)\end{array}$ & $\begin{array}{c}\text { Purification } \\
\text { (fold) }\end{array}$ \\
\hline \multicolumn{6}{|l|}{ BpKatG } \\
\hline Crude extract & 5380 & 2200 & 290 & 100 & 1 \\
\hline $\begin{array}{c}\left(\mathrm{NH}_{4}\right)_{2} \mathrm{SO}_{4} \\
\text { Precipitation }\end{array}$ & 600 & 700 & 980 & 32 & 3 \\
\hline $\begin{array}{l}\text { Anion exchange } \\
\text { (DEAE-A-500) }\end{array}$ & 95 & 360 & 3000 & 16 & 10 \\
\hline $\begin{array}{l}\text { Hyd roxyapatite } \\
\text { column }\end{array}$ & 60 & 200 & 3800 & 9 & 13 \\
\hline \multicolumn{6}{|l|}{ MtKatG } \\
\hline Crude extract & 12300 & 3050 & 250 & 100 & 1 \\
\hline $\begin{array}{c}\left(\mathrm{NH}_{4}\right)_{2} \mathrm{SO}_{4} \\
\text { Precipitation }\end{array}$ & 600 & 830 & 1360 & 27 & 5 \\
\hline $\begin{array}{l}\text { Anion exchange } \\
\text { (DEAE-A-500) }\end{array}$ & 200 & 630 & 3240 & 21 & 13 \\
\hline $\begin{array}{l}\text { Hydroxyapatite } \\
\text { column }\end{array}$ & 130 & 460 & 4450 & 15 & 18 \\
\hline
\end{tabular}


Table 3.2.2b Purification of wild type EcKatG and AfKatG from E. coli strain UM262 harboring plasmid encoded katG of E. coli and A. fulgidus.

\begin{tabular}{|c|c|c|c|c|c|}
\hline Purification Step & $\begin{array}{l}\text { Total } \\
\text { protein } \\
(\mathrm{mg})\end{array}$ & $\begin{array}{l}\text { Total catalase } \\
\text { activity (units } \\
\qquad \times 10^{3} \text { ) }\end{array}$ & $\begin{array}{l}\text { Specific } \\
\text { catalase } \\
\text { activity } \\
\text { (units/mg) }\end{array}$ & $\begin{array}{c}\text { Recovery } \\
(\%)\end{array}$ & $\begin{array}{l}\text { Purification } \\
\text { (fold) }\end{array}$ \\
\hline \multicolumn{6}{|l|}{ EcKatG } \\
\hline Crude extract & 4265 & 6410 & 1500 & 100 & 1 \\
\hline $\begin{array}{c}\left(\mathrm{NH}_{4}\right)_{2} \mathrm{SO}_{4} \\
\text { Precipitation }\end{array}$ & 196 & 420 & 2140 & 7 & 1.4 \\
\hline $\begin{array}{l}\text { Anion exchange } \\
\text { (DEAE-A-500) }\end{array}$ & 73 & 200 & 5050 & 3 & 3.4 \\
\hline $\begin{array}{c}\text { Hydroxyapatite } \\
\text { column }\end{array}$ & 27 & 170 & 6150 & 3 & 4.1 \\
\hline \multicolumn{6}{|l|}{ AfKatG } \\
\hline Crude extract $^{a}$ & 3100 & 2850 & 260 & 100 & 1 \\
\hline $\begin{array}{c}\left(\mathrm{NH}_{4}\right)_{2} \mathrm{SO}_{4} \\
\text { Precipitation }\end{array}$ & 220 & 460 & 2100 & 16 & 8 \\
\hline $\begin{array}{l}\text { Anion exchange } \\
\text { (DEAE-A-500) }\end{array}$ & 70 & 400 & 3500 & 16 & 13 \\
\hline $\begin{array}{c}\text { Hydroxyapatite } \\
\text { column }\end{array}$ & 30 & 380 & 5300 & 13 & 20 \\
\hline
\end{tabular}

\footnotetext{
${ }^{a}$ crude extract was heated at $55^{\circ} \mathrm{C}$ for 30 min after determining catalsae activity
} 
addition of hemin alone increased the catalase specific activity in the whole cells by $\sim 30 \%$. Following these results, hemin chloride was added regularly to the growth medium assuming that its addition allows more efficient incorporation of heme into the overexpressed KatGs. Table 3.2.2a \& b describe the typical purification procedure of BpKatG, MtKatG, EcKatG and AfKatG. The purification procedure is essentially the same for all the KatGs with the exception of the KatG from the thermophile A. fulgidus. In this case the crude extract was heated at $55^{\circ} \mathrm{C}$ for $30 \mathrm{~min}$ in order to precipitate contaminating protein without loss of catalase activity. The difference in the total protein obtained from each enzyme is proportional to the volume of medium used to grow the cells, for example total protein in the crude extract of $\mathrm{MtkatG}$, which was obtained from cells grown in $6 \mathrm{~L}$ of LB was almost four times higher than the total protein in the crude extract of AfkatG which was obtained from cells grown in $2 \mathrm{~L}$ of LB. Passing the protein through hydroxyapatite column also helped in achieving the higher Rz ratio and yield. SyKatG and BsKatG were purified by Mr. Jack Switala, while purified RcKatG was a gift from Dr. Anabella Ivancich from CEA, Saclay, France.

\subsubsection{Polyacrylamide gel electrophoresis (PAGE) of the catalase-peroxidases}

PAGE of purified KatGs under denaturing conditions is shown in Figure 3.1. All the KatGs migrate as a predominantly single bands with an apparent molecular mass $\sim 78$ $\mathrm{kDa}$. A band at approximately $160 \mathrm{kDa}$ appeared in all the lanes and could be attributed to a small amount of slower migrating crosslink dimer which is not reduced by either $\beta$ mercaptoethanol or dithiothreitol added to the sample buffer. In case of EckatG this 160 $\mathrm{kDa}$ band is more prominent presumably because homotetrameric EcKatG resulted in 


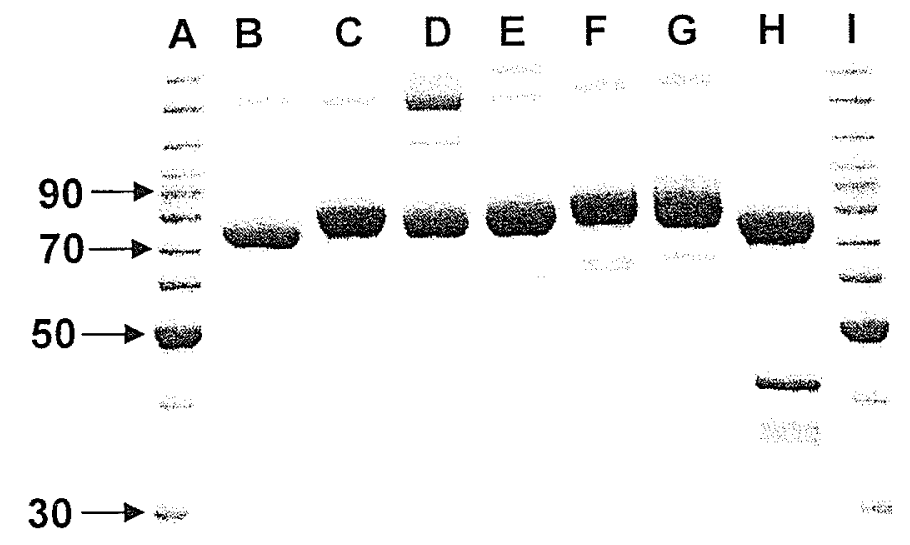

Figure 3.2.1. SDS-polyacrylamide analysis of purified KatGs. Approximately $5 \mu \mathrm{g}$ of sample were run on an $8 \%$ polyacrylamide gel and stained with Coomassie blue. Lanes A-I contains protein MW ladder, BpKatG, MtKatG, EcKatG, SyKatG, BsKatG, AfKatG, RcKatG and protein MW ladder, respectively. 
Table 3.2.3. Comparison of observed optical absorbance maxima, $A_{407 / 280}$ ratio and heme/subunit ratio for purified KatGs.

\begin{tabular}{ccccc}
\hline KatG & $\begin{array}{c}\text { Sorêt } \\
\text { maximum } \\
(\mathrm{nm})\end{array}$ & $\mathrm{A}_{407 / 280 \text { ratio }}$ & $\begin{array}{c}\text { Theoritical } \\
\mathrm{A}_{407 / 280} \text { ratios }^{\mathrm{a}}\end{array}$ & $\begin{array}{c}\text { Heme/subunit } \\
\text { ratio }\end{array}$ \\
\hline BpKatG & 408 & 0.58 & 0.72 & 0.81 \\
MtKatG & 408 & 0.60 & 0.61 & 0.98 \\
EcKatG & 407 & 0.60 & 0.68 & 0.88 \\
SyKatG & 407 & 0.47 & 0.56 & 0.84 \\
BsKatG & 406 & 0.47 & 0.58 & 0.81 \\
AfkatG & 404 & 0.54 & 0.60 & 0.90 \\
RcKatG & 406 & 0.52 & 0.66 & 0.79 \\
\hline
\end{tabular}

${ }^{\mathrm{a}} \varepsilon$ at $280 \mathrm{~nm}$ calculated based on $\varepsilon_{\mathrm{TRP}}=5500$ and $\varepsilon_{\mathrm{TYR}}=1490, \varepsilon$ at $407 \mathrm{~nm}$ for heme estimated to be $100.000 \mathrm{M}^{-1} \mathrm{~cm}^{-1}$ 
Figure 3.2.2. Absorption spectra of KatGs. The left axis is for the range from 350 to 500 $\mathrm{nm}$ while the right axis is for the range from 500 to $700 \mathrm{~nm}$. 

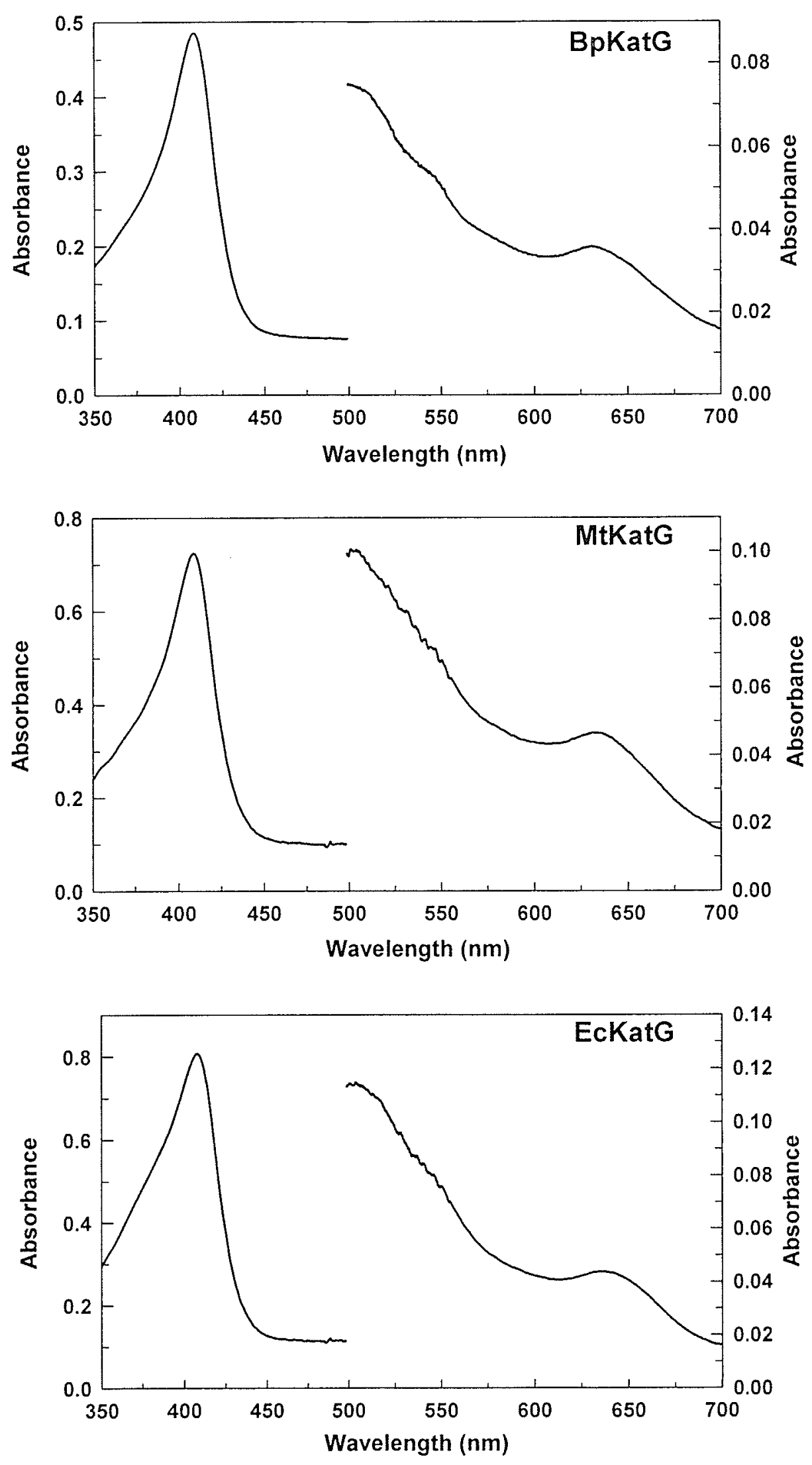

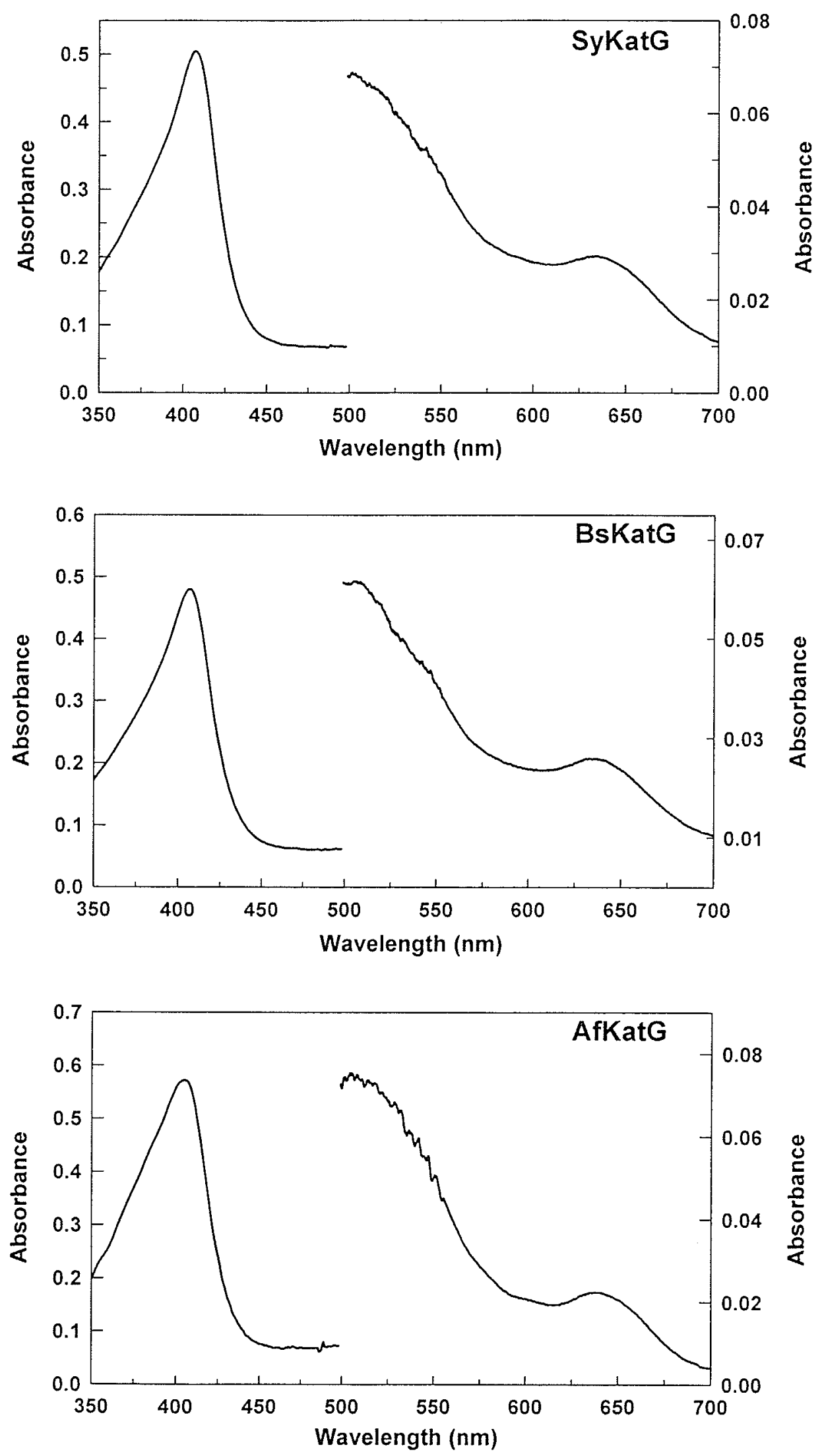


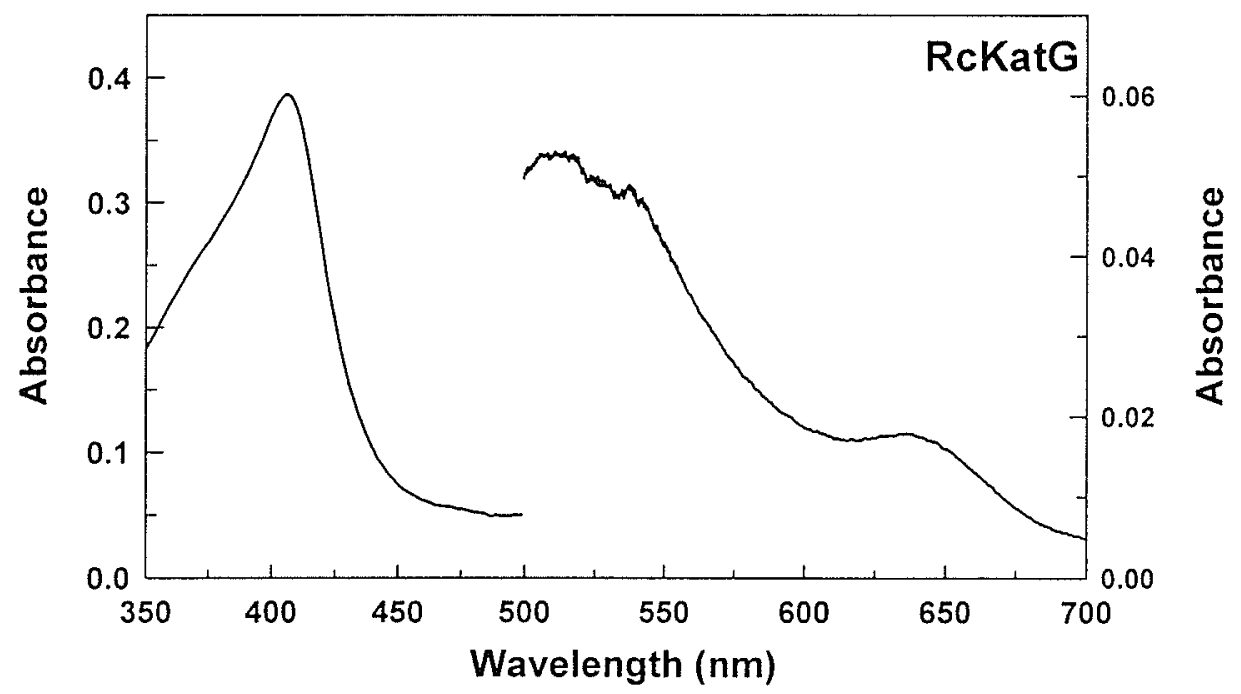


higher dimer formation as compared to the rest of the homodimeric KatGs. In case of $\mathrm{RcKatG}$, a single band at $\sim 46 \mathrm{kDa}$ is also visible without any explanation.

\subsubsection{UV-visible absorption spectroscopy of catalase-peroxidases}

Absorbance spectra of the purified KatGs in the Soret and visible regions of the spectrum are shown in Figure 3.2.2 while the positions of absorption maxima, heme/protein ratio (A Sorêt $/ 280$ ) and heme/subunit ratio has been summarized in Table 3.2.3. There is a variation of $4 \mathrm{~nm}$ between the seven KatGs in the Sorêt band with BpKatG and MtKatG showing Sorêt band at $408 \mathrm{~nm}$ whearas AfKatG having the same at

$404 \mathrm{~nm}$. EckatG and SyKatG have a Sorêt band at $407 \mathrm{~nm}$ and RckatG and BsKatG show Sorêt band at $406 \mathrm{~nm}$. The spectra in 500-700 nm region show the charge transfer (CT) bands at $\sim 500$ (CT 1) and $\sim 630 \mathrm{~nm}$ (CT 2). These charge transfer bands generally indicate the changes in the hydrogen bonding network in the vicinity of the heme. Purified KatGs showed a heme/protein $\left(\mathrm{A}_{407 / 280}\right)$ ratio or the $\mathrm{Rz}$ value of 0.5 or higher with heme/subunit occupancy $>0.8$, maximum being 1.0 for MtKatG, suggesting better heme incorporation.

\subsection{4. $\mathrm{pH}$ profile for the catalase and peroxidase activities}

The $\mathrm{pH}$ profiles for the catalase and peroxidase activities of the KatGs are shown in Figure 3.3. All KatGs show different $\mathrm{pH}$ optimum for catalase and peroxidase activities. The $\mathrm{pH}$ optima for peroxidase activity are between $\mathrm{pH} 4$ and 5 for all seven KatGs using ABTS as peroxidatic substrate whereas catalatic pH optimum is between 6.25 and 6.5 as shown in Table 3.2.4. 
Table 3.2.4. $\mathrm{pH}$ optima for catalatic and peroxidatic activities of KatGs

\begin{tabular}{ccc}
\hline & \multicolumn{2}{c}{$\mathrm{pH}$ Optimum } \\
\cline { 2 - 3 } KatG & Peroxidase activity & Catalase activity \\
(ABTS) & 6.5 \\
BpKatG & 4.5 & 6.5 \\
MtKatG & 4.75 & 6.25 \\
EcKatG & 4.25 & 6.5 \\
SyKatG & 4.25 & 6.5 \\
BsKatG & 4 & 6.25 \\
AfkatG & 4.5 & 6.5 \\
RcKatG & 5 & \\
\hline
\end{tabular}


Figure 3.2.3. $\mathrm{pH}$ profile for catalase and peroxidase activities of KatGs 

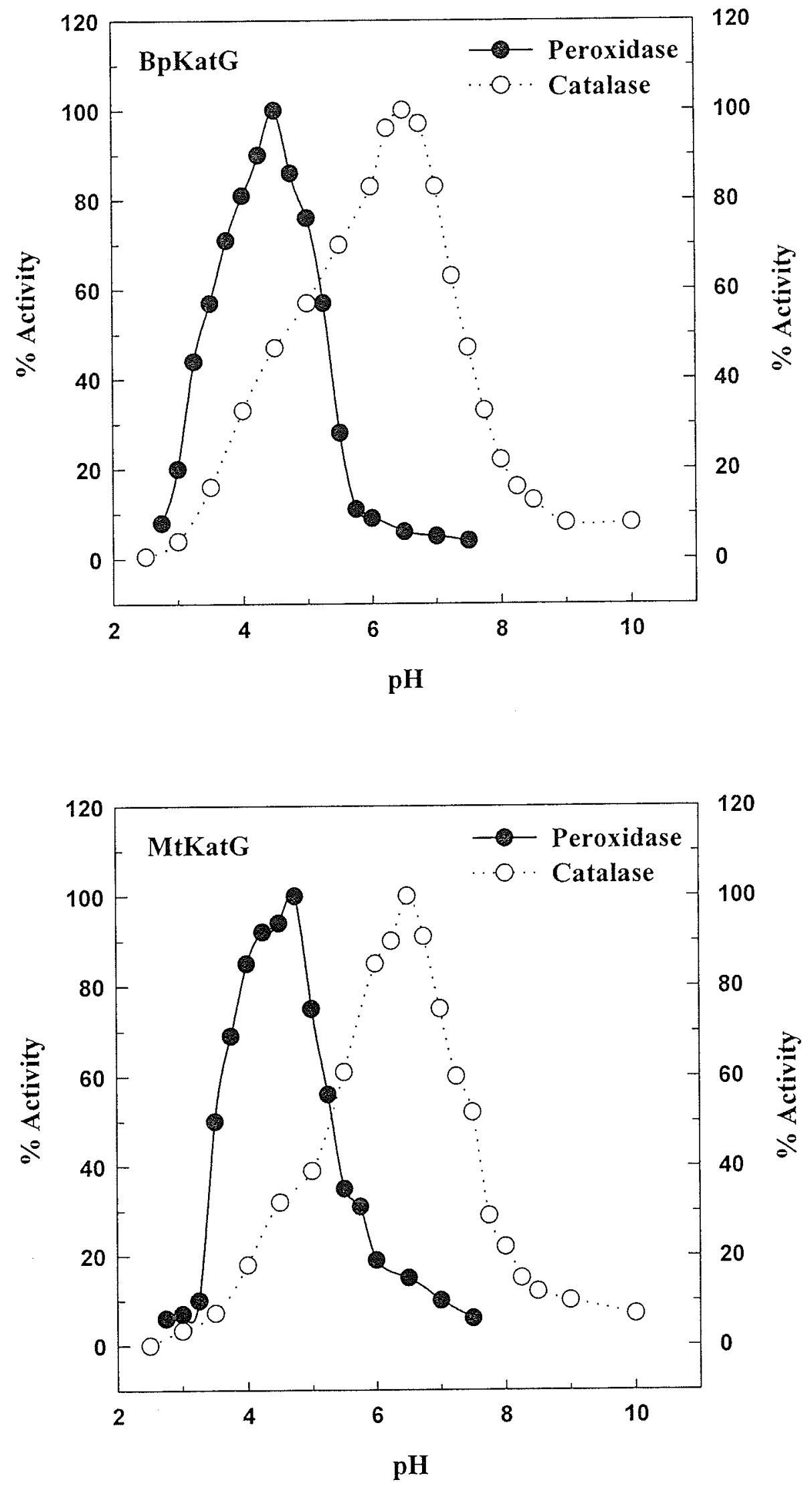

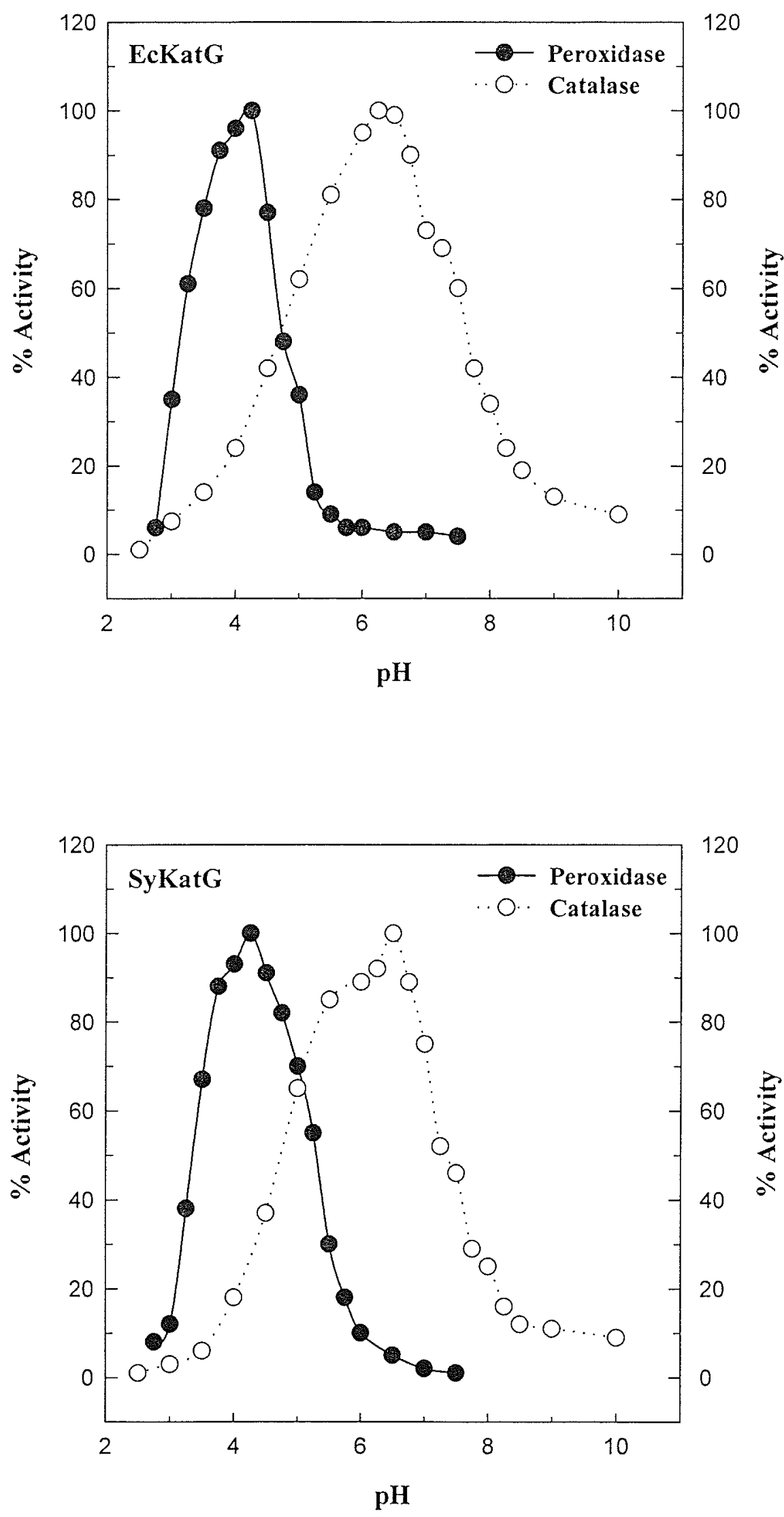

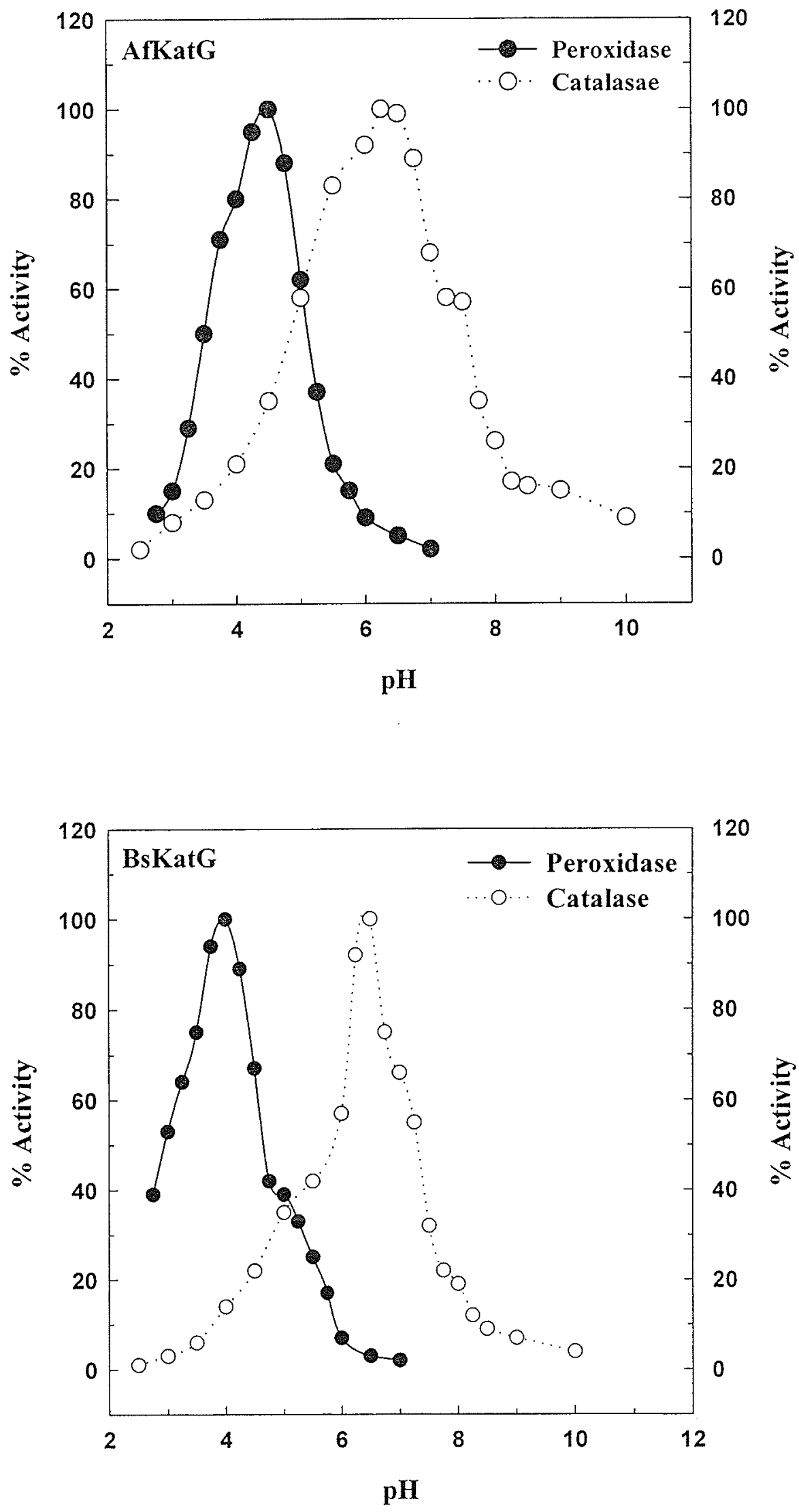


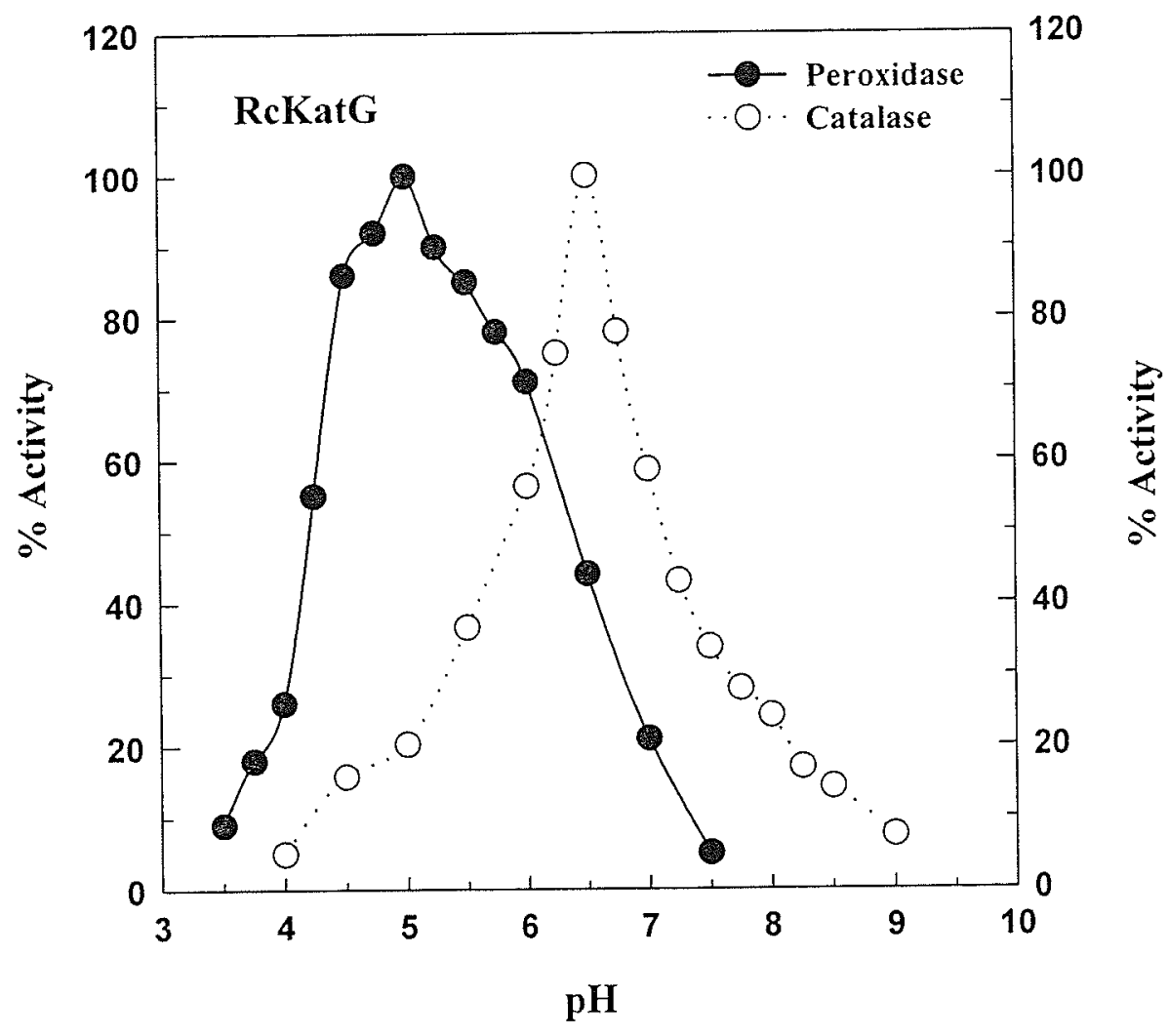




\subsubsection{Specific catalase and peroxidase activities of KatGs}

Table 3.2.5 summarizes the specific enzymatic activities of KatGs. All the KatGs show substantial catalase activity ranging from $3634 \mathrm{U} / \mathrm{mg}$ by BpKatG to $5985 \mathrm{U} / \mathrm{mg}$ by EcKatG. The latter shows significantly higher activity from the previous reports where the activity was reported from EcKatG produced without exogenous addition of hemin in the growth medium. Peroxidase activity was determined using two different organic electron donors (ABTS and $o$-dianisidine) as peroxidatic substrate. All the KatGs show a broad specificity in peroxidase activity ranging from $2 \mathrm{U} / \mathrm{mg}$ for RcKatG to $11.7 \mathrm{U} / \mathrm{mg}$ for AfkatG using ABTS as substrate and for o-dianisidine ranging from $1 \mathrm{U} / \mathrm{mg}$ for RckatG to $8.8 \mathrm{U} / \mathrm{mg}$ for MtKatG.

\subsubsection{Kinetic Characterization of KatGs}

Kinetic constants for the catalase and peroxidase reactions of seven KatGs were determined by titrating enzyme with different concentrations of substrate. Figure 3.4 shows the effect of various concentrations of $\mathrm{H}_{2} \mathrm{O}_{2}$ on the rate of catalase reaction of KatGs. The initial velocities shown are expressed as $\mu$ moles of $\mathrm{H}_{2} \mathrm{O}_{2}$ decomposed per min per $\mu$ mole of heme. The kinetic constants for catalase activity are shown in Table 3.2.6. Kinetic constants for peroxidase activity, using ABTS as an electron donor, were determined by titrating KatGs against concentrations of ABTS and results are expressed as $\mu$ mole of ABTS oxidized per min per $\mu$ mole of heme as shown in Figure 3.5 and the kinetic constants are shown in Table 3.2.7.

Both catalase and peroxidase reactions require $\mathrm{H}_{2} \mathrm{O}_{2}$ for compound I formation. In order to determine the kinetic constants for compound I formation during peroxidase 
Table 3.2.5. Comparison of catalase and peroxidase activities of KatGs

\begin{tabular}{|c|c|c|c|}
\hline \multirow[b]{2}{*}{ KatG } & \multirow{2}{*}{ 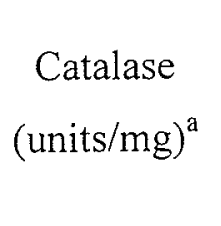 } & \multicolumn{2}{|c|}{ Peroxidase } \\
\hline & & $\begin{array}{c}\text { ABTS } \\
\text { (units/mg) }^{\text {a }}\end{array}$ & $\begin{array}{c}\text {-dianisidine } \\
{\text { (units } / \mathrm{mg})^{\mathrm{a}}}\end{array}$ \\
\hline BpKatG & $3630 \pm 150$ & $4.8 \pm 0.9$ & $5.3 \pm 0.9$ \\
\hline MtKatG & $4450 \pm 150$ & $10 \pm 2.5$ & $8.8 \pm 1.5$ \\
\hline EcKatG & $5990 \pm 180$ & $11 \pm 1.3$ & $8.3 \pm 2.1$ \\
\hline SyKatG & $5150 \pm 500$ & $6.4 \pm 2.1$ & $5.5 \pm 2.1$ \\
\hline BsKatG & $3690 \pm 380$ & $5.9 \pm 1.6$ & $5.3 \pm 2.0$ \\
\hline AfkatG & $5280 \pm 250$ & $12 \pm 3.3$ & $3.2 \pm 0.6$ \\
\hline RcKatG & $5520 \pm 226$ & $2.0 \pm 0.3$ & $1.0 \pm 0.2$ \\
\hline
\end{tabular}

${ }^{\mathrm{a}}(1$ unit $=1 \mu \mathrm{mol} / \mathrm{min})$ 
Figure 3.2.4. Effect of $\mathrm{H}_{2} \mathrm{O}_{2}$ concentration on the initial catalatic velocities of $\mathrm{KatGs}$ 


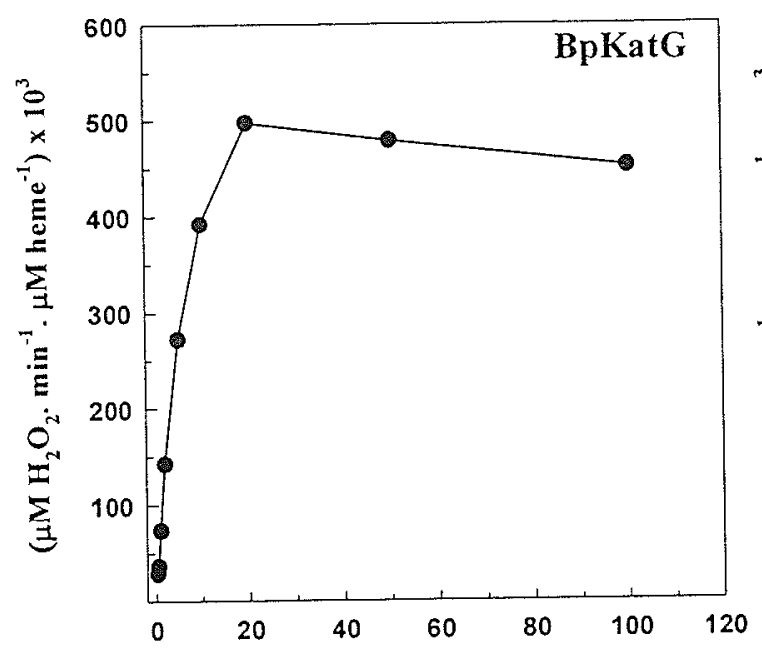

$\left[\mathrm{H}_{2} \mathrm{O}_{2}\right] \mathrm{mM}$
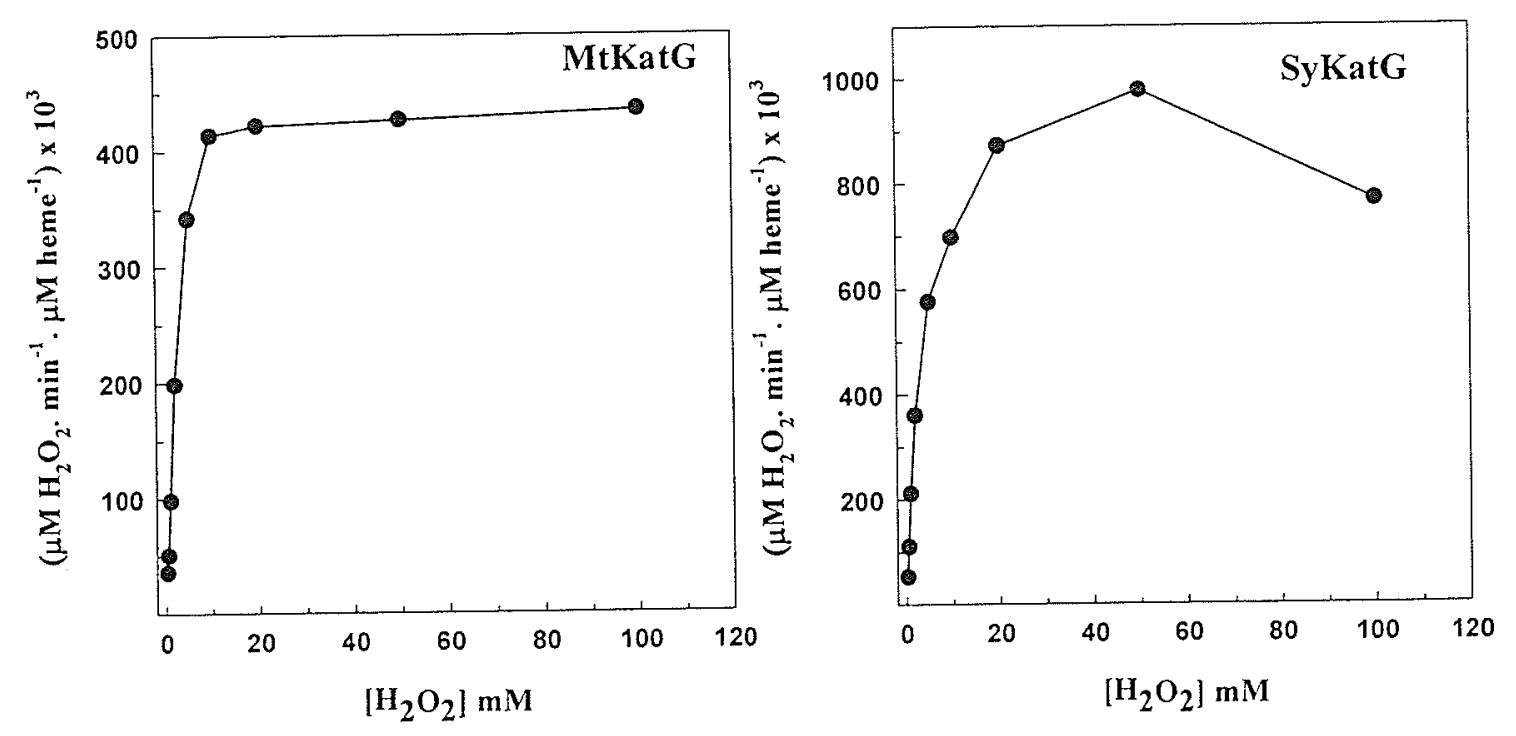

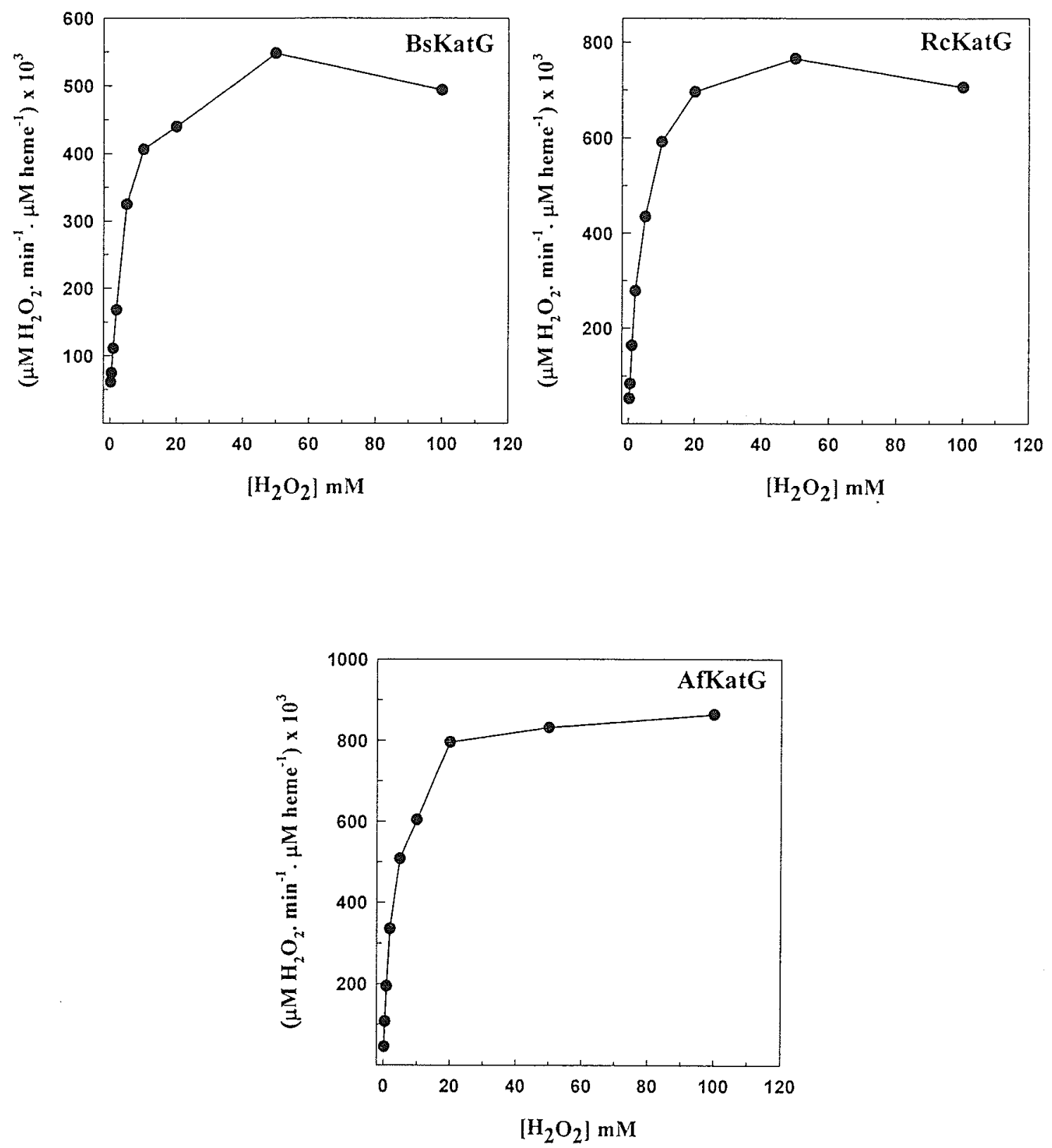
Table 3.2.6. Comparison of the observed catalatic kinetic parameters of purified KatGs, using $\mathrm{H}_{2} \mathrm{O}_{2}$ as substrate.

\begin{tabular}{ccccc}
\hline KatG & $\begin{array}{c}\mathrm{V}_{\max }{ }^{2} \\
\left(\times 10^{3}\right)\end{array}$ & $\begin{array}{c}K_{\mathrm{m}} \\
(\mathrm{mM})\end{array}$ & $\begin{array}{c}k_{\text {cat }} \\
\left(\mathrm{s}^{-1}\right)\end{array}$ & $\begin{array}{c}k_{\text {cat }} / K_{\mathrm{m}} \\
\left(\mathrm{M}^{-1} \mathrm{~s}^{-1}\right)\end{array}$ \\
\hline BpKatG & $525 \pm 29$ & $4.3 \pm 0.9$ & $8750 \pm 483$ & $2.0 \times 10^{6}$ \\
MtKatG & $455 \pm 22$ & $2.4 \pm 0.5$ & $7600 \pm 367$ & $3.2 \times 10^{6}$ \\
EcKatG & $800 \pm 32$ & $4.2 \pm 0.8$ & $13400 \pm 543$ & $3.2 \times 10^{6}$ \\
SyKatG & $1010 \pm 50$ & $3.1 \pm 0.7$ & $17300 \pm 837$ & $5.6 \times 10^{6}$ \\
BsKatG & $540 \pm 19$ & $3.7 \pm 0.7$ & $9050 \pm 317$ & $2.5 \times 10^{6}$ \\
AfKatG & $900 \pm 19$ & $3.8 \pm 0.3$ & $14960 \pm 317$ & $3.9 \times 10^{6}$ \\
RcKatG & $820 \pm 22$ & $3.7 \pm 0.9$ & $13600 \pm 367$ & $3.7 \times 10^{6}$ \\
\hline
\end{tabular}

${ }^{\mathrm{a}} \mathrm{V}_{\max }$ is expressed as $\mu$ moles of $\mathrm{H}_{2} \mathrm{O}_{2}$ decomposed $\min ^{-1} \mu$ mole heme ${ }^{-1}$ 
Figure 3.2.5. Effect of ABTS concentration on the initial peroxidatic velocities of KatGs. 

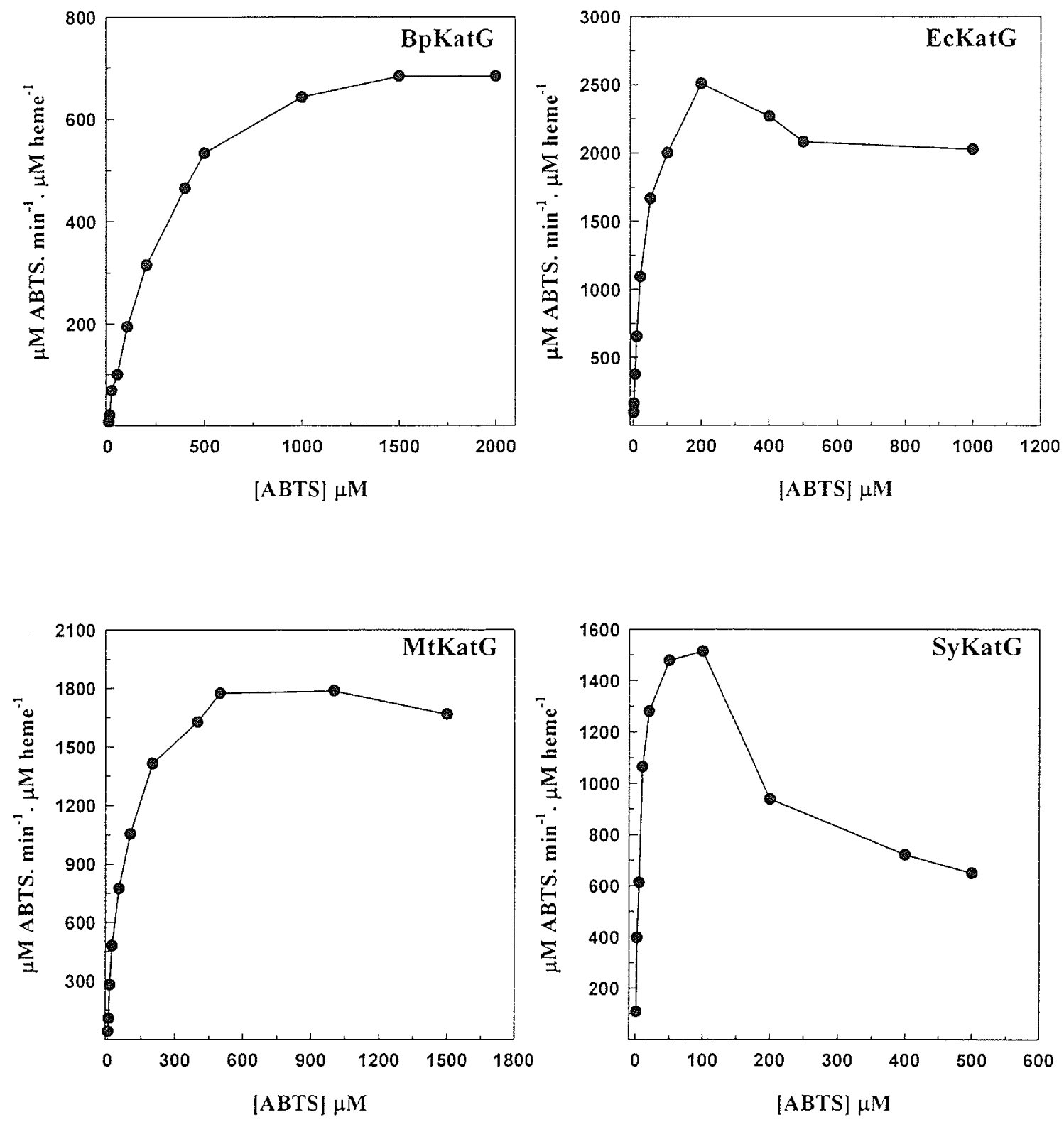

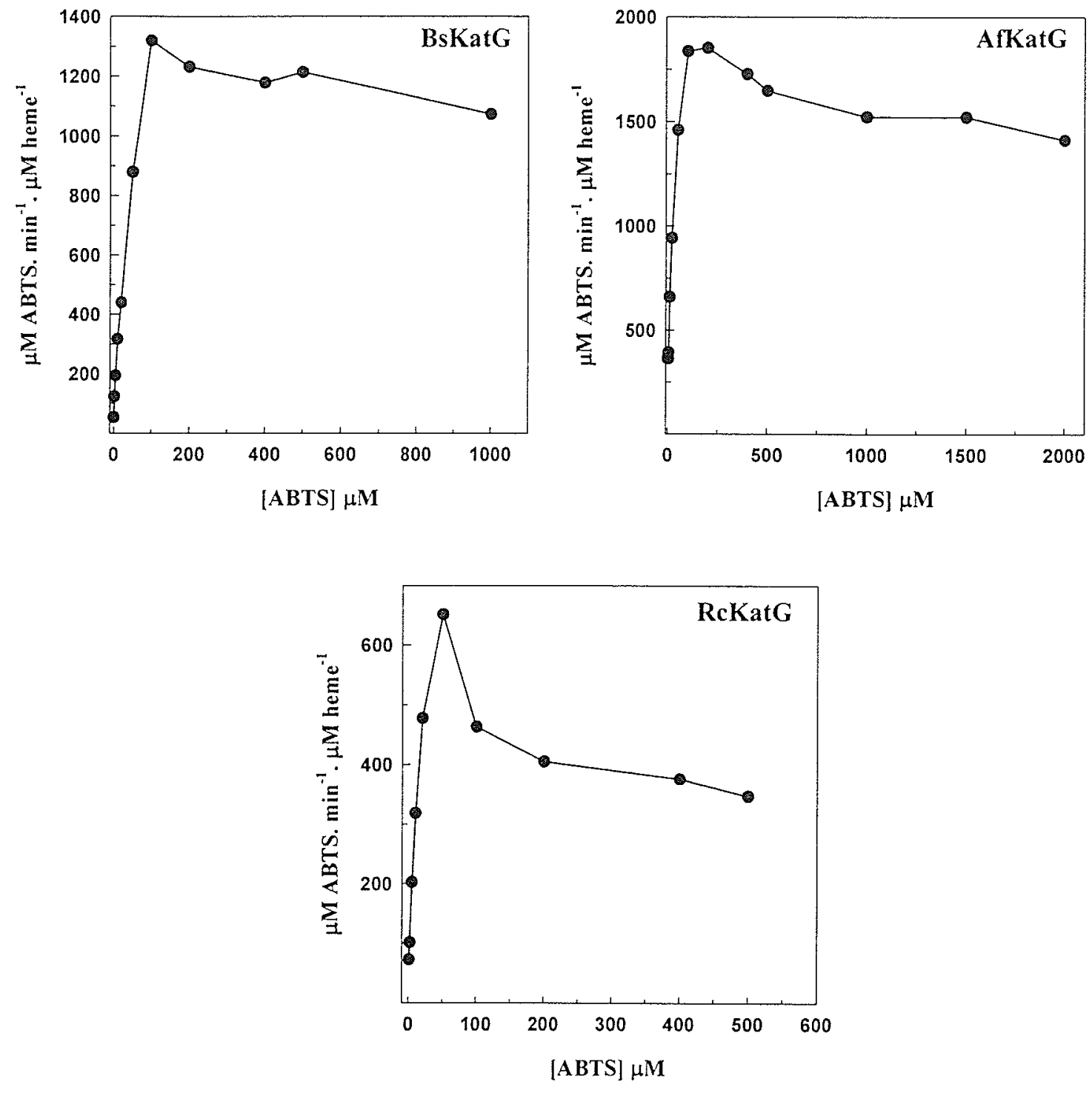
Table 3.2.7 Comparison of the observed peroxidatic kinetic parameters of purified KatGs, using ABTS as substrate.

\begin{tabular}{ccccc}
\hline KatG & $\mathrm{V}_{\max }{ }^{\mathrm{a}}$ & $\begin{array}{c}K_{\mathrm{m}} \\
(\mu \mathrm{M})\end{array}$ & $\begin{array}{c}k_{\text {cat }} \\
\left(\mathrm{s}^{-1}\right)\end{array}$ & $\begin{array}{c}k_{\text {cat }} / K_{\mathrm{m}} \\
\left(\mathrm{M}^{-1} \mathrm{~s}^{-1}\right)\end{array}$ \\
\hline BpKatG & $810 \pm 14$ & $300 \pm 21$ & $14 \pm 0.2$ & $0.5 \times 10^{5}$ \\
MtKatG & $1870 \pm 53$ & $67 \pm 7$ & $31 \pm 0.9$ & $4.5 \times 10^{5}$ \\
EcKatG & $2450 \pm 107$ & $24 \pm 5$ & $41 \pm 1.8$ & $17 \times 10^{5}$ \\
SyKatG & $1680 \pm 72$ & $7 \pm 1$ & $28 \pm 1.2$ & $40 \times 10^{5}$ \\
BsKatG & $1420 \pm 105$ & $31 \pm 8$ & $24 \pm 1.8$ & $7.6 \times 10^{5}$ \\
AfKatG & $1870 \pm 94$ & $16 \pm 4$ & $31 \pm 1.6$ & $19 \times 10^{5}$ \\
RcKatG & $890 \pm 30$ & $16 \pm 1$ & $15 \pm 0.5$ & $9.3 \times 10^{5}$ \\
\hline
\end{tabular}

${ }^{\mathrm{a}} \mathrm{V}_{\max }$ is expressed as $\mu$ moles of ABTS oxidized $\mathrm{min}^{-1} \mu$ mole heme ${ }^{-1}$ 
Figure 3.2.6. Effect of $\mathrm{H}_{2} \mathrm{O}_{2}$ concentration on the initial peroxidatic velocities of KatGs. 

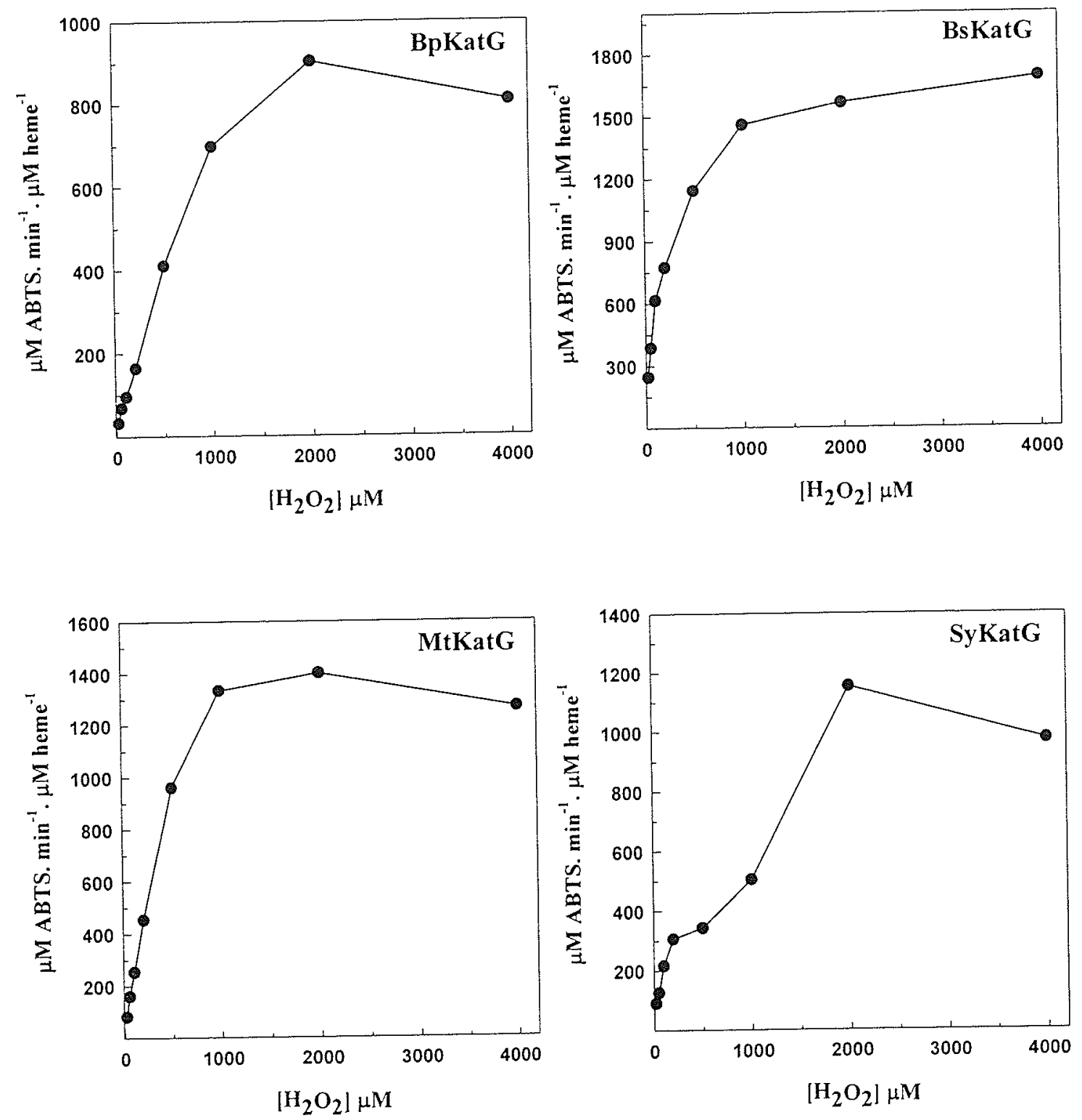

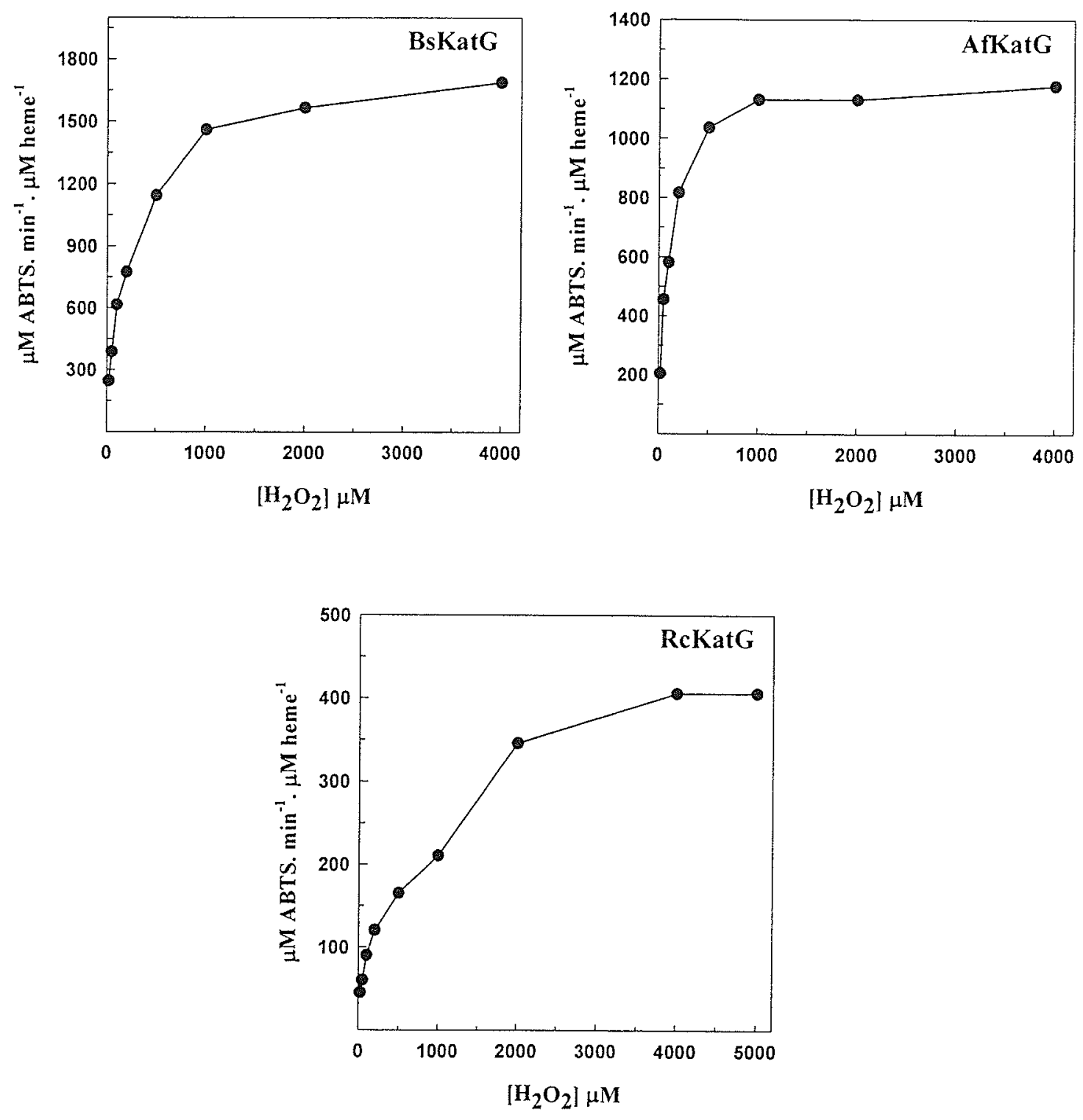
Table 3.2.8 Comparison of the observed peroxidatic kinetic parameters of purified $\mathrm{KatGs}$, using $\mathrm{H}_{2} \mathrm{O}_{2}$ as substrate for the compound I formation.

\begin{tabular}{ccccc}
\hline KatG & $\mathrm{V}_{\max }{ }^{\mathrm{a}}$ & $\begin{array}{c}K_{\mathrm{m}}{ }^{\mathrm{b}} \\
(\mu \mathrm{M})\end{array}$ & $\begin{array}{c}k_{\mathrm{cat}} \\
\left(\mathrm{s}^{-1}\right)\end{array}$ & $\begin{array}{c}k_{\text {cat }} / K_{\mathrm{m}} \\
\left(\mathrm{M}^{-1} \mathrm{~s}^{-1}\right)\end{array}$ \\
\hline BpKatG & $1070 \pm 110$ & $700 \pm 220$ & $18 \pm 1.8$ & $0.3 \times 10^{5}$ \\
MtKatG & $1570 \pm 120$ & $360 \pm 100$ & $26 \pm 1.7$ & $0.7 \times 10^{5}$ \\
EcKatG & $2080 \pm 160$ & $60 \pm 20$ & $35 \pm 2.7$ & $6.0 \times 10^{5}$ \\
SyKatG & $1440 \pm 54$ & $1000 \pm 60$ & $24 \pm 1$ & $0.2 \times 10^{5}$ \\
BsKatG & $1740 \pm 53$ & $210 \pm 27$ & $29 \pm 1$ & $1.4 \times 10^{5}$ \\
AfKatG & $1210 \pm 9$ & $95 \pm 6$ & $20 \pm 0.2$ & $2.1 \times 10^{5}$ \\
RcKatG & $470 \pm 60$ & $830 \pm 80$ & $8 \pm 1$ & $0.1 \times 10^{5}$ \\
\hline
\end{tabular}

${ }^{\mathrm{a}} \mathrm{V}_{\text {max }}$ is expressed as $\mu$ moles of ABTS oxidized $\min ^{-1} \mu$ mole heme ${ }^{-1}$

${ }^{b} \mathrm{~K}_{\mathrm{m}}$ is shown for $\mathrm{H}_{2} \mathrm{O}_{2}$ 
activity of KatGs, initial velocities for peroxidase activity were determined at various concentrations of $\mathrm{H}_{2} \mathrm{O}_{2}$ as shown in Figure 3.2.6, the kinetic constants are summarized in the table 3.2.8. At lower substrate concentrations (100 to $200 \mathrm{mM}$ ), KatGs follow Michaelis-Menten kinetics for catalase reaction while higher concentrations tend to inhibit the enzyme activity as shown in Figure 3.2.4. The $K m$ value for all seven KatGs studied was found to be in milimolar range, between 2.4 and 4.8 , with a very high turnover rate $\left(k_{\text {cat }}\right)$.

Figure 3.2.5 shows that KatGs follow Michaelis-Menten kinetics for peroxidase activity even at higher concentration of substrate (ABTS). The exceptions to this were SyKatG and RcKatG which showed significant inhibition in the activity at higher substrate (ABTS) concentration. The $K_{\mathrm{m}}$ values for ABTS (Figure 3.2.7.) were determined in $\mu$ molar range for all seven KatGs but the variantion among enzymes was prominent ranging from $300 \mu \mathrm{M}(\mathrm{BpKatG})$ to $7 \mu \mathrm{M}$ (SyKatG). The turnover rate or $k_{\text {cat }}$ values also were much lower for peroxidsae reaction as compared to catalase reaction. Figure 3.2.6 shows that KatGs follow Michaelis-Menten kinetics for compound I formation with an exception of SyKatG and RcKatG both of which show significant increase in the initial velocity at $\mathrm{H} 2 \mathrm{O} 2$ concentration higher that $1 \mathrm{mM}$. The $K_{\mathrm{m}}$ values for $\mathrm{H}_{2} \mathrm{O}_{2}$ are found in $\mu$ molar range except for SyKatG which showed a high $K_{\mathrm{m}}$ of $\sim 1$ $\mathrm{mM}$.

\subsubsection{Effect of heme inhibitor on catalase activity of KatGs}

Cyanide and azide are classical heme protein inhibitors that bind to the active-site heme iron reversibly to form stable complexes. Figure 3.2.7 compares the effect of $\mathrm{KCN}$ 

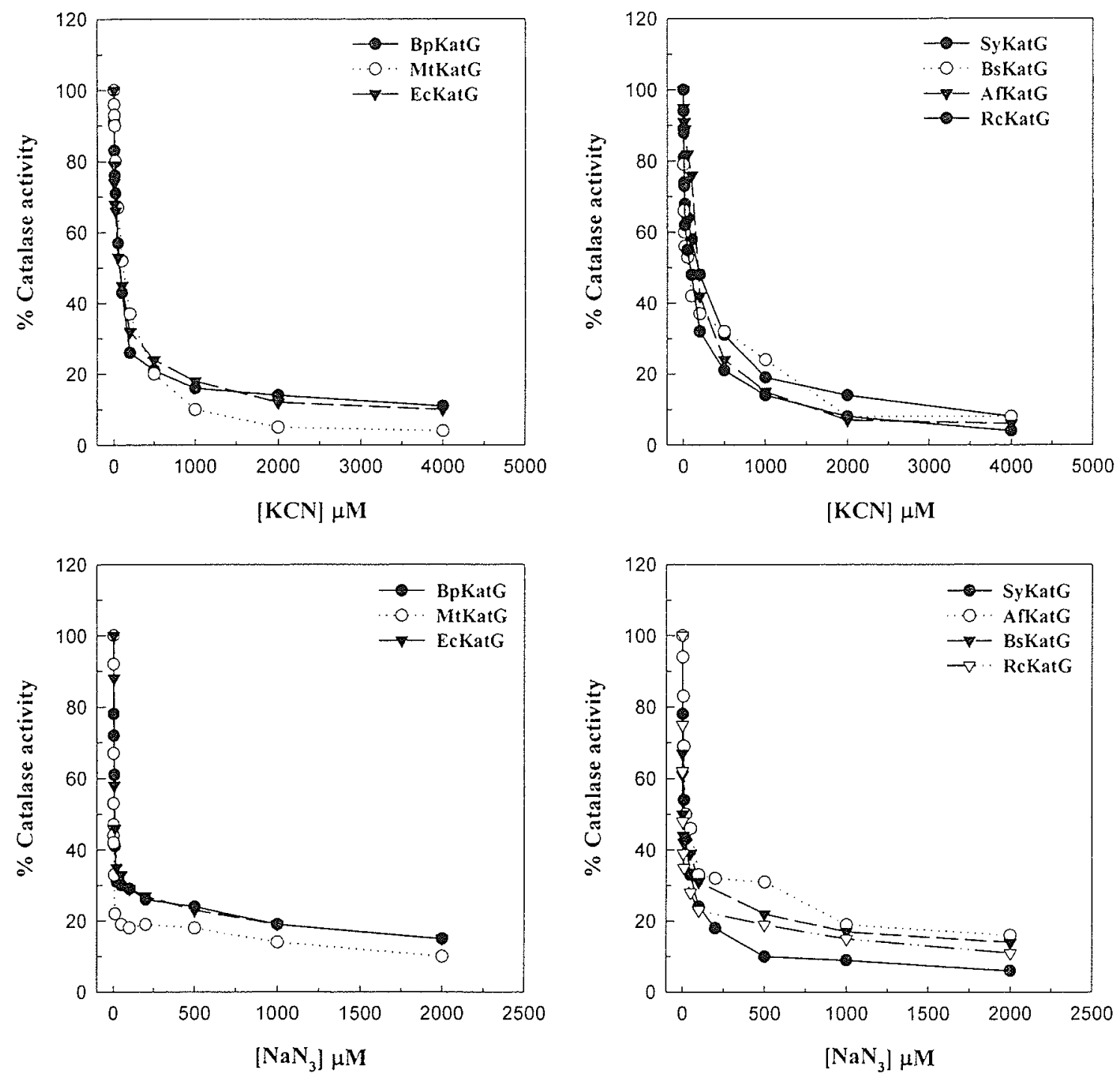

Figure 3.2.7. Comparison of the sensitivity of $\mathrm{KatGs}$ to cyanide $(\mathrm{KCN})$ or azide $\left(\mathrm{NaN}_{3}\right)$. 
Table 3.2.9. Comparison of the sensitivity of $\mathrm{KatGs}$ to cyanide $(\mathrm{KCN})$ or azide $\left(\mathrm{NaN}_{3}\right)$.

\begin{tabular}{|c|c|c|}
\hline KatG & $\begin{array}{c}{[\mathrm{KCN}] \text { causing } 50 \%} \\
\text { inhibition }(\mu \mathrm{M})\end{array}$ & $\begin{array}{c}{\left[\mathrm{NaN}_{3}\right] \text { causing } 50 \%} \\
\text { inhibition }(\mu \mathrm{M})\end{array}$ \\
\hline BpKatG & 40 & 8 \\
\hline MtKatG & 100 & 0.2 \\
\hline EcKatG & 50 & 7 \\
\hline SyKatG & 170 & 13 \\
\hline BsKatG & 50 & 2 \\
\hline AfKatG & 150 & 20 \\
\hline RcKatG & 80 & 8 \\
\hline
\end{tabular}


reversibly to form stable complexes. Figure 3.7 compares the effect of $\mathrm{KCN}$ and $\mathrm{NaN}_{3}$ on the catalase activity of $\mathrm{KatGs}$ and the $\mathrm{IC}_{50}$ for both $\mathrm{KCN}$ and $\mathrm{NaN}_{3}$ are summarized in Table 3.9. Both $\mathrm{KCN}$ and $\mathrm{NaN}_{3}$ show similar inhibition patterns for catalase activity but the $\mathrm{IC}_{50}$ for $\mathrm{KCN}$ is higher than that of $\mathrm{NaN}_{3}$ though, it remains in the $\mu$ molar region. 


\subsection{KatGs and isoniazid activation}

Isonicotinic acid hydrazide (isoniazid or $\mathrm{INH}$ ) is a widely used pro-drug effective against Mycobacterium tuberculosis (Deretic et al., 1996). Formation of isonicotinoyl$\mathrm{NAD}$, the active form of the drug, involves removal of hydrazine from INH by KatG (Zhang et al., 1992) and ligation of the isonicotinoyl group with $\mathrm{NAD}^{+}$(Johnsson et al., 1995). Isonicotinoyl-NAD interferes with the synthesis of mycolic acid and therefore cell wall synthesis by binding to InhA, an enoyl-acyl carrier protein reductase (Rozwarski $e t$ al., 1998), and possibly to KasA, a $\beta$-ketoacyl acyl carrier protein synthase (Mdluli et al., 1998), blocking their NADH-binding sites. The central role of KatG in INH activation is evident in the significant fraction of INH-resistant cases of tuberculosis attributable to mutations in $k a t G$ and in biochemical studies that have demonstrated a direct role for KatG in the generation of various isonicotinoyl derivatives (Johnsson and Schultz, 1994). Much of the literature related to the activation of INH by KatG has focused on the fate of INH and possible intermediates involved in the process (Johnsson and Schultz, 1994; Wilming and Johnsson, 1995; Magliozzo and Marcinkeviciene, 1996). With NAD included in the mix, the generation of the isonicotinoyl-NAD adduct was observed both with and without KatG present (Wilming and Johnsson, 1995; Magliozzo and Marcinkeviciene, 1996; Lie et al., 2000), leading to the suggestions that the role of KatG is limited to the hydrazine lyase of INH and that the subsequent reaction of the isonicotinoyl radical with $\mathrm{NAD}^{+}$is a nonenzymatic event involving a homolytic aromatic substitution (Wilming and Johnsson, 1995; Minisci, et al., 1989). Reactive oxygen species have been implicated in INH activation both in vivo (Mitchison and Selkon, 1956; Youatt, 1959) and in vitro (Johnsson and Schultz, 1994), and an elevated level of 
superoxide (Wang, et al., 1998) was identified as a possible reason for the high sensitivity of M. tuberculosis to INH (Deretci et al., 1996). However, the absence of $\mathrm{H}_{2} \mathrm{O}_{2}$ involvement in $\mathrm{NHH}$ activation implies that a reaction different from either the peroxidase or the catalase reactions is involved, and some reports have suggested that the active participation of KatG in INH activation involves more than just hydrazine lyase (Lei et al., 2000). During this study a comparative analysis of their interaction with INH and some other pyridine derivatives such as NADH, NADPH and pyridoxine revealed striking peculiarities of KatGs.

\subsubsection{Catalase-peroxidases (KatG) exhibit NADH oxidase activity}

NADH oxidases are widely distributed in nature, being found in species ranging from bacteria (Ashrafuddin and Claiborne, 1989; Zoldak et al., 2003) and archaebacteria (Kengen et al., 2003) to mammals (Xia et al., 2003). The enzymes can be both soluble and membrane-bound, and although NADH is the common electron donor, water, hydrogen peroxide, or superoxide may be formed as products depending on the enzyme.

The physiological role of NADH oxidases in many instances is unknown, but the reaction has been described as a detoxifier of oxygen in nitrogen fixing organisms and archaebacteria (Kengen et al., 2003) and as a possible sensor of oxygen levels in the regulation of muscle contractility (Xia et al., 2003). A variation of the NADH oxidase reaction, a "peroxidase-oxidase" reaction has been well characterized in horseradish peroxidase (HRP) to require a catalytic amount of $\mathrm{H}_{2} \mathrm{O}_{2}$ that is alternately used and regenerated during the reaction of NAD radicals with molecular oxygen (Akazawa and Conn, 1958; Yokota and Yamazaki, 1965; Yokota and Yamazaki, 1977; Dunford, 1999). 
Plant peroxidases, including HRP, have about $20 \%$ sequence similarity and remarkable

structural similarity to the $\mathrm{N}$-terminal domain of $\mathrm{KatG}$ proteins, particularly in the vicinity of the heme active site, raising the possibility that $\mathrm{KatG}$ proteins may also exhibit an NADH peroxidase-oxidase activity. Indeed, there are reports of NADH being oxidized to $\mathrm{NAD}^{+}$by $\mathrm{KatG}$ in both peroxidase (Ro et al., 2003) and an oxidase-like (Wilming and Johnsson, 1999) reactions, but the reaction of $\mathrm{KatG}$ with $\mathrm{NADH}$ has not been characterized. Keeping in mind the potential importance of any reaction involving NADH in isonicotinoyl-NAD formation, the interactions of KatG with NADH was investigated and an oxygen-dependent NADH oxidase activity was demonstrated along with a direct role for $\mathrm{KatG}$ in the formation of isonicotinoyl-NAD.

\subsubsection{NADH Oxidation by KatG}

$\mathrm{NAD}^{+}$replaces the hydrazine moiety of the anti-tubercular pro-drug INH to generate the active form of the drug, isonicotinoyl-NAD. In an attempt to define the role of KatG in the formation of isonicotinoyl-NAD, the potential utilization of NADH and $\mathrm{NAD}^{+}$as substrates was investigated, revealing that NADH supports BpKatG-mediated radical generation (Figure 3.3.1a) in the absence of $\mathrm{H}_{2} \mathrm{O}_{2}$ using NBT as radical sensor at a rate approximately equal to the rate of NADH disappearance (Figure 3.3.1b). A similar comparison of other KatGs used in this study demonstrated a similar fate of NADH but at a much slower rate as compared to BpKatG (Table 3.3.1 \& Figure 3.3.1). In all cases the radical formation, using NBT as radical sensor, can be clearly differentiated from the background activity of the control lacking KatG. Among KatGs, RcKatG, MtKatG and SyKatG show substantial rates of radical production from a reaction mixture containing 
Table 3.3.1. Comparison of NADH oxidase activity of KatGs

\begin{tabular}{ccc}
\hline KatG & $\begin{array}{c}\text { NADH oxidase @ } \triangle 340 \mathrm{~nm} \\
\text { pmole NADH. } \mathrm{min}^{-1} \cdot \mathrm{nmol}^{-1}\end{array}$ & $\begin{array}{c}\text { NADH oxidase @ 560nm } \\
\text { pmole NBT. } \mathrm{min}^{-1} \cdot \mathrm{nmol}^{-1}\end{array}$ \\
\hline BpKatG & $510 \pm 85$ & $640 \pm 96$ \\
MtKatG & $30 \pm 3$ & $13 \pm 4$ \\
EcKatG & $15 \pm 6$ & $80 \pm 6$ \\
SyKatG & $10 \pm 3$ & $60 \pm 6$ \\
BsKatG & $6 \pm 6$ & $65 \pm 4$ \\
AfkatG & $38 \pm 6$ & $240 \pm 20$ \\
RcKatG & $30 \pm 9$ &
\end{tabular}


Figure 3.3.1. NADH oxidation by catalase-peroxidases. $a$, the rates of radical production in a reaction mixture containing $100 \mu \mathrm{M} \mathrm{NADH}, 1.2 \mu \mathrm{M} \mathrm{KatG}, 200 \mu \mathrm{M}$ NBT and 50 $\mathrm{mM}$ Tris, $\mathrm{pH} 8.75$, were followed by formazan appearance measured at $560 \mathrm{~nm}$. Separate assays contain no enzyme (control), BpKatG, MtKatG, EcKatG, SyKatG, BsKatG, AfKatG, \& RcKatG. $b$, the rates of NADH oxidation in a solution containing $100 \mu \mathrm{M}$ $\mathrm{NADH}, 1.2 \mu \mathrm{M} \mathrm{KatG}$, and $50 \mathrm{mM}$ Tris, pH 8.75 were followed by NADH disappearance measured at $340 \mathrm{~nm}$. Separate assays contain no enzyme (control), BpKatG, MtKatG, EcKatG, SyKatG, BsKatG, AfKatG, \& RcKatG. 


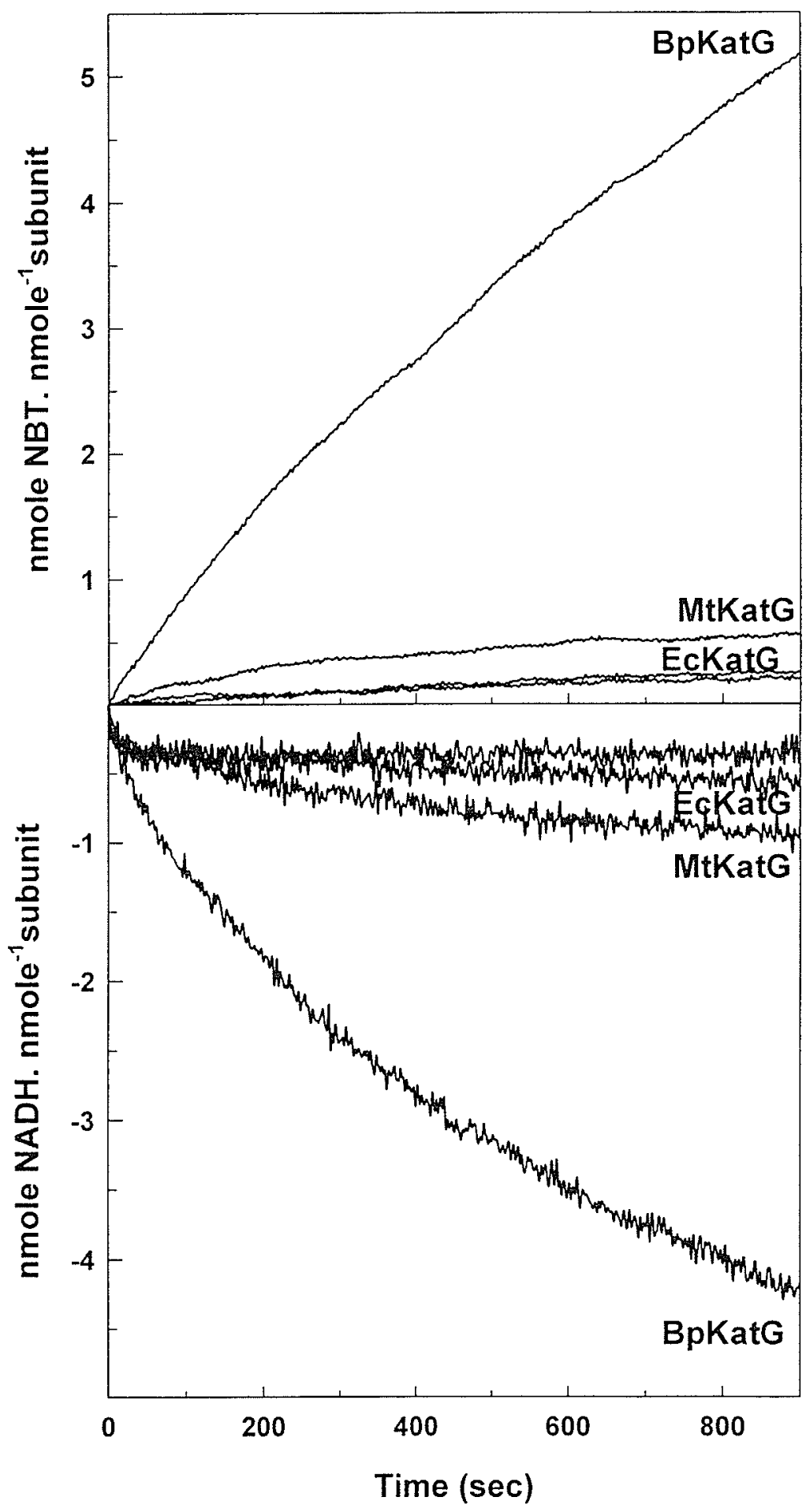




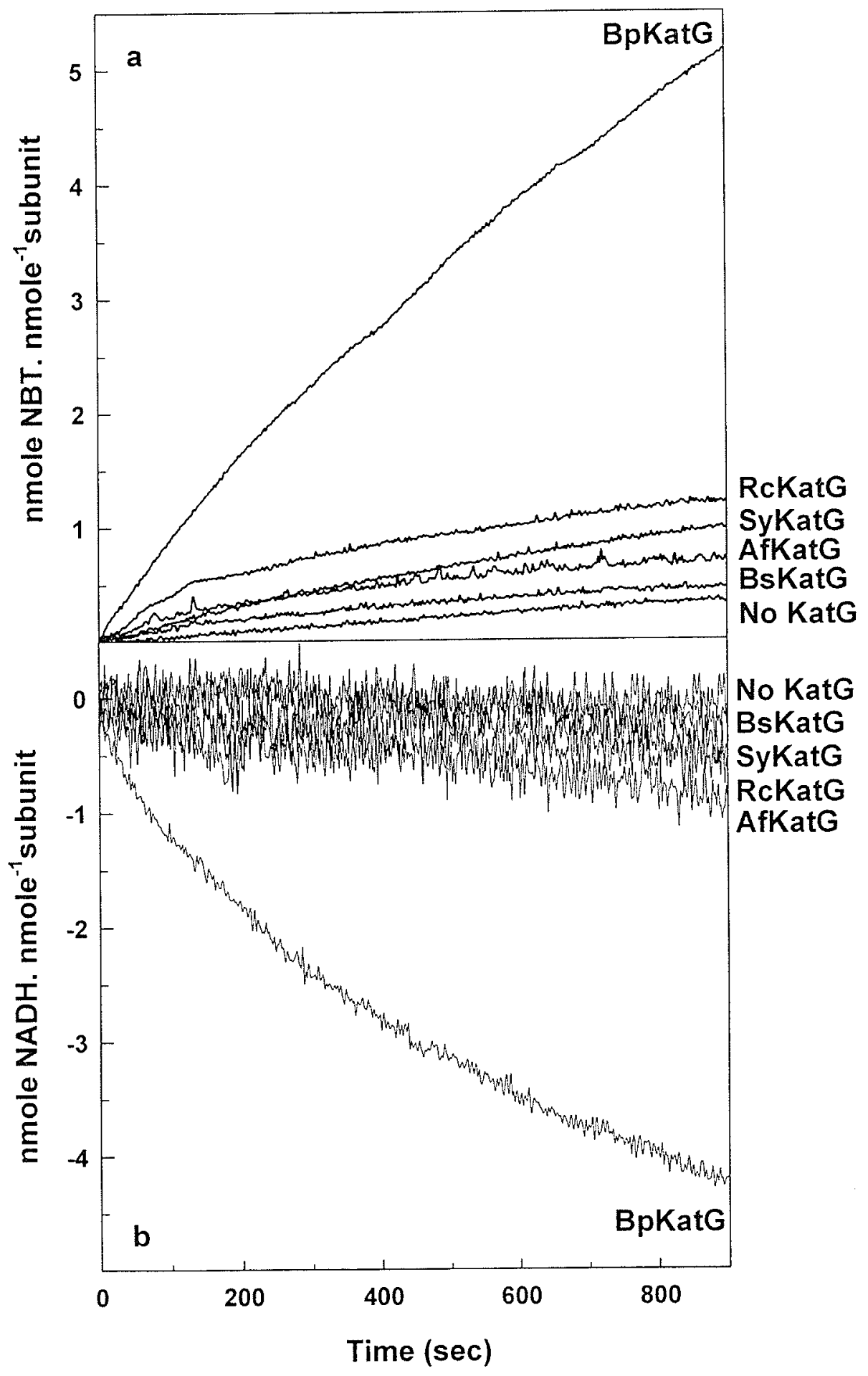




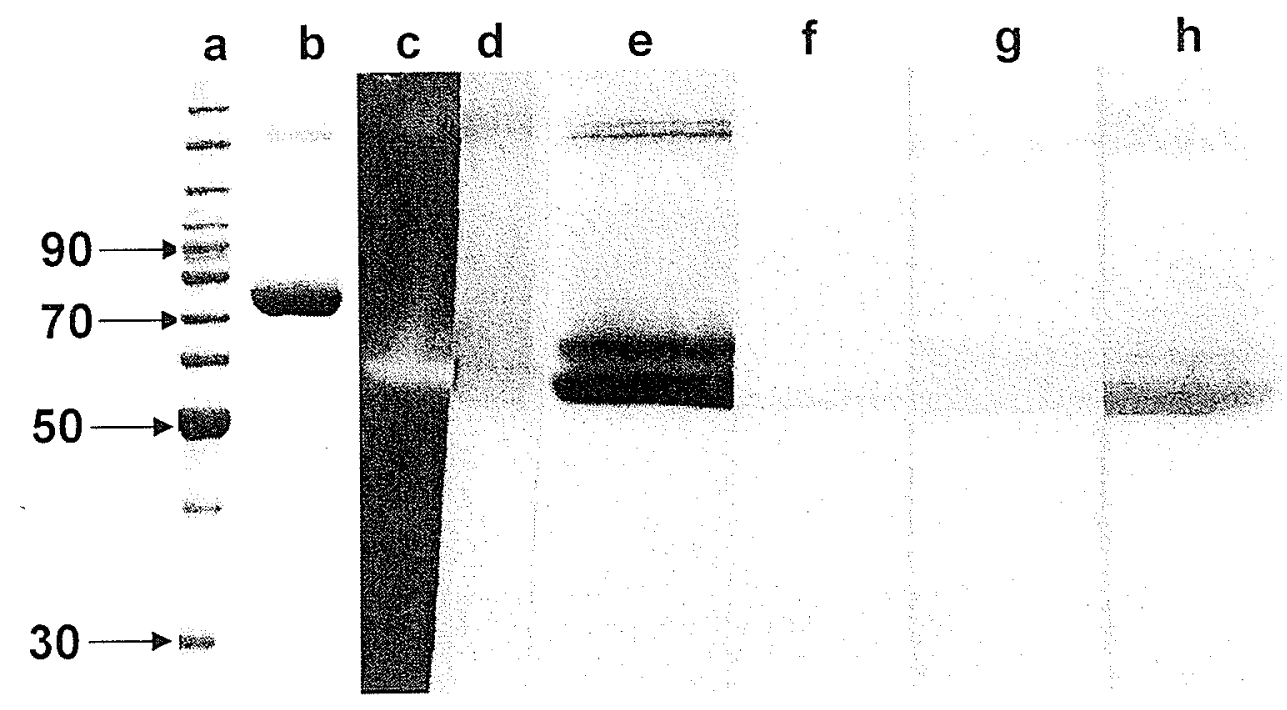

Figure 3.3.2. Migration of purified BpKatG on a $8 \%$ SDS-polyacrylamide gel (lane a and $b$ ) and a nondenaturing $8 \%$ polyacrylamide gel (lanes $c-h$ ). Lane $b$ (SDS-

polyacrylamide gel) was stained with Coomassie Brilliant Blue dye. A single $200 \mu \mathrm{g}$ amount of $\mathrm{BpKatG}$ was loaded in one large lane for lanes $c-h$, and after electrophoresis, the gel was cut into six strips for separate staining. Lane $c$ was stained for catalase activity (a clear band on a brown background). Lane $d$ was stained for peroxidase activity (brown bands on a clear background). Lane e was stained with Coomassie Brilliant Blue. Lane $f$ was stained for oxidase activity in a mixture of $200 \mu \mathrm{M} \mathrm{NADH}$ and $200 \mu \mathrm{M}$ NBT. Lane $g$ was stained for INH hydrazine lyase activity in a mixture of 10 $\mathrm{mM}$ INH and $200 \mu \mathrm{M}$ NBT. Lane $h$ was stained for combined INH hydrazine lyase and NADH oxidase activity in a mixture of $10 \mathrm{mM} \mathrm{NNH}, 200 \mu \mathrm{M} \mathrm{NADH}$, and $200 \mu \mathrm{M}$ NBT. 
Table 3.3.2. KatG-mediated radical production in the presence of different substrates

\begin{tabular}{|c|c|c|c|c|}
\hline & No enzyme $\mathrm{e}^{\mathrm{a}}$ & BpKatG ${ }^{b}$ & MtKatG $^{\mathrm{b}}$ & EcKatG $^{b}$ \\
\hline & pmol. min $^{-1}$ & \multicolumn{3}{|c|}{ pmole. min $^{-1}$. nmole ${ }^{-1}$ subunit } \\
\hline NADH & $3.0 \pm 0.2$ & $640 \pm 96$ & $100 \pm 15$ & $13 \pm 4$ \\
\hline $\mathrm{NADH}+\mathrm{Mn}^{2+}$ & $2.0 \pm 0.1$ & $540 \pm 23$ & $99 \pm 4$ & $10 \pm 4$ \\
\hline $\mathrm{NADH}-\mathrm{O}_{2}^{\mathrm{c}}$ & $1.0 \pm 0.2$ & $320 \pm 15$ & $9 \pm 2$ & $7 \pm 2$ \\
\hline $\mathrm{NADH}+\mathrm{SOD}$ & $1.0 \pm 0.1$ & $350 \pm 8$ & $58 \pm 6$ & $5 \pm 2$ \\
\hline NADPH & $1.0 \pm 0.1$ & $260 \pm 2$ & $1.0 \pm 0.1$ & $1.0 \pm 0.1$ \\
\hline $\mathrm{NAD}^{+}$ & $3.0 \pm 0.6$ & $\mathrm{ND}^{\mathrm{d}}$ & $N D^{d}$ & $N D^{d}$ \\
\hline $\mathbf{N A D H}+\mathbf{I N H}$ & $8.0 \pm 2$ & $1140 \pm 76$ & $260 \pm 21$ & $100 \pm 6$ \\
\hline $\mathrm{NADH}+\mathrm{INH}+\mathrm{Mn}^{2+}$ & $290 \pm 16$ & $2940 \pm 29$ & $830 \pm 17$ & $930 \pm 91$ \\
\hline $\mathrm{NAD}^{+}+\mathbf{I N H}$ & $4.0 \pm 0.3$ & $82 \pm 1$ & $79 \pm 8$ & $74 \pm 19$ \\
\hline $\mathrm{NADH}+\mathrm{INH}+\mathrm{Mn}^{+2}$ & $140 \pm 4$ & $230 \pm 12$ & $340 \pm 5$ & $220 \pm 9$ \\
\hline INH & $5 \pm 0.2$ & $76 \pm 2$ & $78 \pm 9$ & $38 \pm 2$ \\
\hline $\mathbf{I N H}+\mathbf{M n}^{2+}$ & $190 \pm 18$ & $410 \pm 3$ & $460 \pm 5$ & $450 \pm 2$ \\
\hline $\mathrm{INH}-\mathrm{O}_{2}{ }^{\mathrm{c}}$ & $6 \pm 2$ & $75 \pm 5$ & $78 \pm 5$ & $40 \pm 3$ \\
\hline $\mathrm{INH}+\mathrm{SOD}$ & $5.2 \pm 1$ & $90 \pm 2$ & $65 \pm 5$ & $38 \pm 3$ \\
\hline INH + pyridoxine & $4.0 \pm 1$ & $9.0 \pm 2$ & $20 \pm 5$ & $18 \pm 3$ \\
\hline
\end{tabular}

${ }^{\text {a }}$ All the reactions contained $200 \mu \mathrm{M}$ NBT in $50 \mathrm{mM}$ Tris pH 8.75 or pH 8.0 (for reactions involving $\mathrm{NH}$ ), supplemented with $10 \mathrm{mM} \mathrm{NNH}, 250 \mu \mathrm{M} N A D H, 250 \mu \mathrm{M}$ $\mathrm{NADPH}$, and $250 \mu \mathrm{M} \mathrm{NAD}{ }^{+}$, and $200 \mu \mathrm{M}$ of superoxide dismutase as indicated. $\mathrm{O}_{2}$ was reduced by flushing the reaction mixture with $\mathrm{N}_{2}$ gas.

${ }^{\mathrm{b}} \mathrm{KatG}$ was added $1.2 \mu \mathrm{M}$.

${ }^{c}$ For anaerobic reactions, the reaction mixtures were flushed with nitrogen to remove dissolve oxygen.

${ }^{\mathrm{d}} \mathrm{ND}$, not detected 
NADH, NBT and KatG as well as upon direct observation of NADH disappearance at $340 \mathrm{~nm}$ (Figure 3.3.1 a and b) and BsKatG shows the lowest level of NADH oxidase activity at both 560 and $340 \mathrm{~nm}$. NADPH supports a slower rate of radical generation, and $\mathrm{NAD}^{+}$does not support any radical production (Table 3.3.2). $\mathrm{NAD}^{+}$was confirmed as the product of the reaction with NADH by HPLC analysis (Figure 3.3.4). Both superoxide dismutase and a lower molecular oxygen concentration reduce radical generation (Table 3.3.2) from NADH. The pH optima for both radical production and NADH disappearance are 8.75 , but the curves are not perfectly superimposable (Figure 3.3.3), suggesting two different, $\mathrm{pH}$-dependent, fates for the reduced oxygen, most likely $\mathrm{H}_{2} \mathrm{O}_{2}$ at lower $\mathrm{pH}$ and superoxide radical at higher $\mathrm{pH}$. Unfortunately, the rapid degradation of $\mathrm{H}_{2} \mathrm{O}_{2}$ by both the catalase and peroxidase activities of $\mathrm{BpKatG}$ precluded its identification or even the observation of any spectral changes in the enzyme during the reaction. The optimum $\mathrm{pH}$ for the oxidase reaction is significantly different from the optimum $\mathrm{pH}$ levels for the peroxidase $(\mathrm{pH} 4.5)$ and catalase $(\mathrm{pH}$ 6.5) reactions (Figure 3.3.3). These results are consistent with BpKatG having an NADH oxidase activity producing $\mathrm{NAD}^{+}$and either superoxide ion or $\mathrm{H}_{2} \mathrm{O}_{2}$ and with a similar activity existing in MtKatG, EcKatG, SyKatG, BsKatG, AfKatG and RcKatG, but at a much lower level.

\subsubsection{Visualization of NADH oxidase activity by PAGE under non-denaturating conditions.}

Purified BpKatG migrates as a single band (apparent mass of $78 \mathrm{kDa}$ ) on denaturing gels (Figure 3.3.2, lane a) with only a small amount of slower migrating dimer. On a nondenaturing gel (Figure 3.3.1, lanes $b-g$ ), catalase, peroxidase, and 


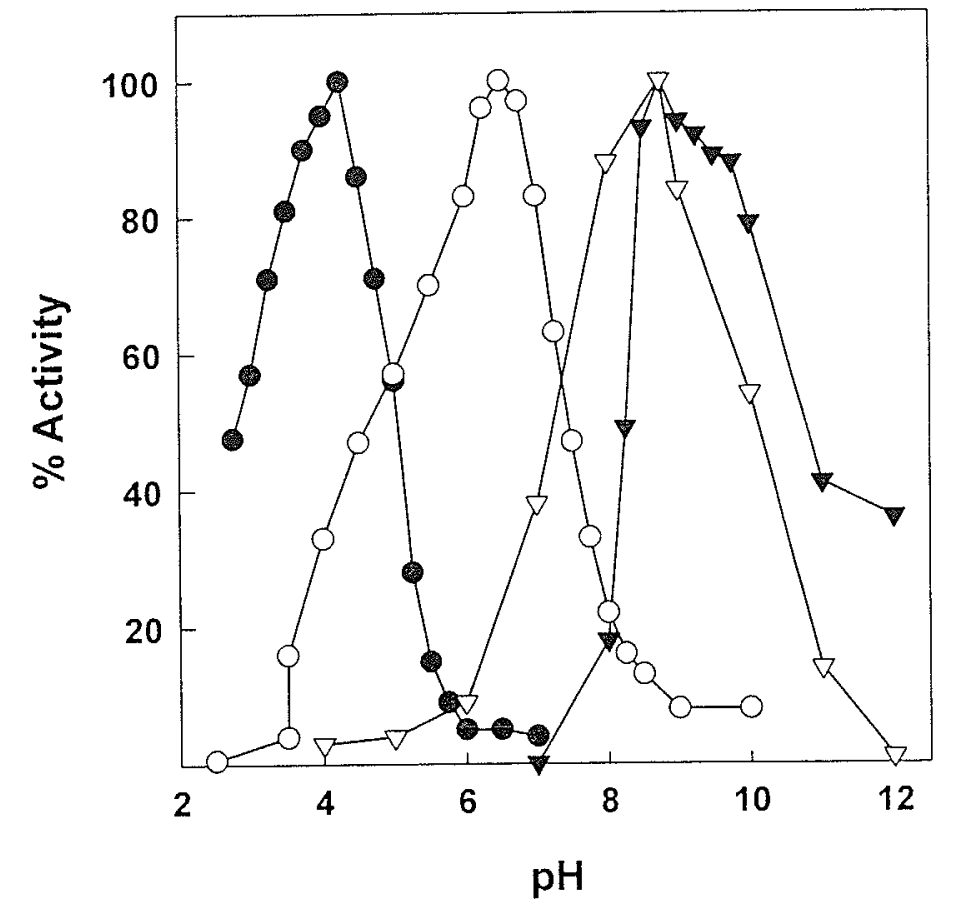

Figure 3.3.3. $\mathrm{pH}$ dependence of $\mathrm{NADH}$ oxidation by $\mathrm{BpKatG}$ determined by radical formation at $560 \mathrm{~nm}(\nabla)$ and NADH disappearance at $340 \mathrm{~nm}(\nabla)$ compared with the $\mathrm{pH}$ dependence of the catalase $(\mathrm{O})$ and peroxidase $(0$ ) reactions. Buffered solutions contained $50 \mathrm{mM}$ each of sodium acetate, $\mathrm{pH} \mathrm{3,4,5}$; potassium phosphate, $\mathrm{pH} 6$ and 7; Tris-HCL, pH 8 and 9; and CHES, pH 10. 
Figure 3.3.4. Elution profiles from reverse phase HPLC of reaction products from mixtures containing $100 \mu \mathrm{M} \mathrm{NADH}$ and no enzyme (a); $1.2 \mu \mathrm{M} \mathrm{BpKatG}$ (b); $1.2 \mu \mathrm{M}$ $\mathrm{MtKatG}$ (c); $1.2 \mu \mathrm{M}$ EcKatG (d). $N-H, \mathrm{NADH} ; N-D, \mathrm{NAD}^{+}$. 


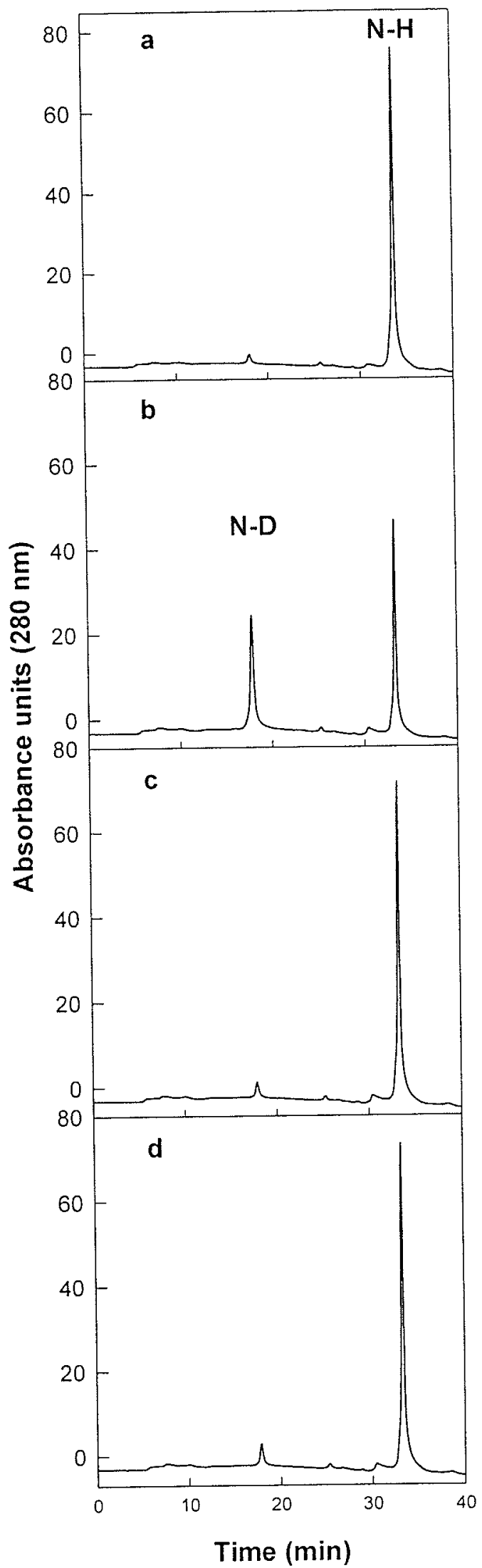


oxidase activities all co-migrate with the main bands of protein (lanes $b-e$ ). The presence of two bands of catalase-peroxidase with the same mass on nondenaturing gels has been noted previously but not explained.

\subsubsection{Kinetic characterization of NADH oxidase activity}

Titration of BpKatG with different concentrations of NADH demonstrates that BpKatG follows Michaelis-Menton kinetics for the NADH oxidase reaction (Figure 3.3.5). The kinetic parameters (Table 3.3.3) reveal a relatively high affinity $\left(K_{\mathrm{m}}\right)$ for NADH but a very slow turnover rate $\left(k_{\text {cat }}\right)$ in comparison with the catalase and peroxidase reactions. $\mathrm{NAD}^{+}$and pyridoxine act as competitive inhibitors of the NADH oxidase reaction (Table 3.3.2 \& Figure 3.3.6).

\subsubsection{Comparison of NADH oxidase activity with NADH peroxidase-oxidase} reaction by HRP

HRP catalyzes an NADH peroxidase-oxidase reaction in which $\mathrm{H}_{2} \mathrm{O}_{2}$ is required to initiate the reaction, after which there is a cycling of $\mathrm{O}_{2}{ }^{\circ-}, \mathrm{H}_{2} \mathrm{O}_{2}$, and compound III (Dunford, 1999). Given the sequence similarity between plant peroxidases and catalaseperoxidases, particularly in the active site, the possibility that the NADH oxidase activity of $\mathrm{KatG}$ is similar to the peroxidase-oxidase reaction was investigated (Table 3.3.4). The HRP peroxidase-oxidase reaction is characterized by a need for a catalytic amount of $\mathrm{H}_{2} \mathrm{O}_{2}$, by inhibition by $\mathrm{SOD}$ or catalase, and by long lag periods in the presence of low

NADH concentrations and high HRP concentrations. NADH oxidation by BpKatG does not exhibit anyof these characteristics (Table 3.3.4). For 

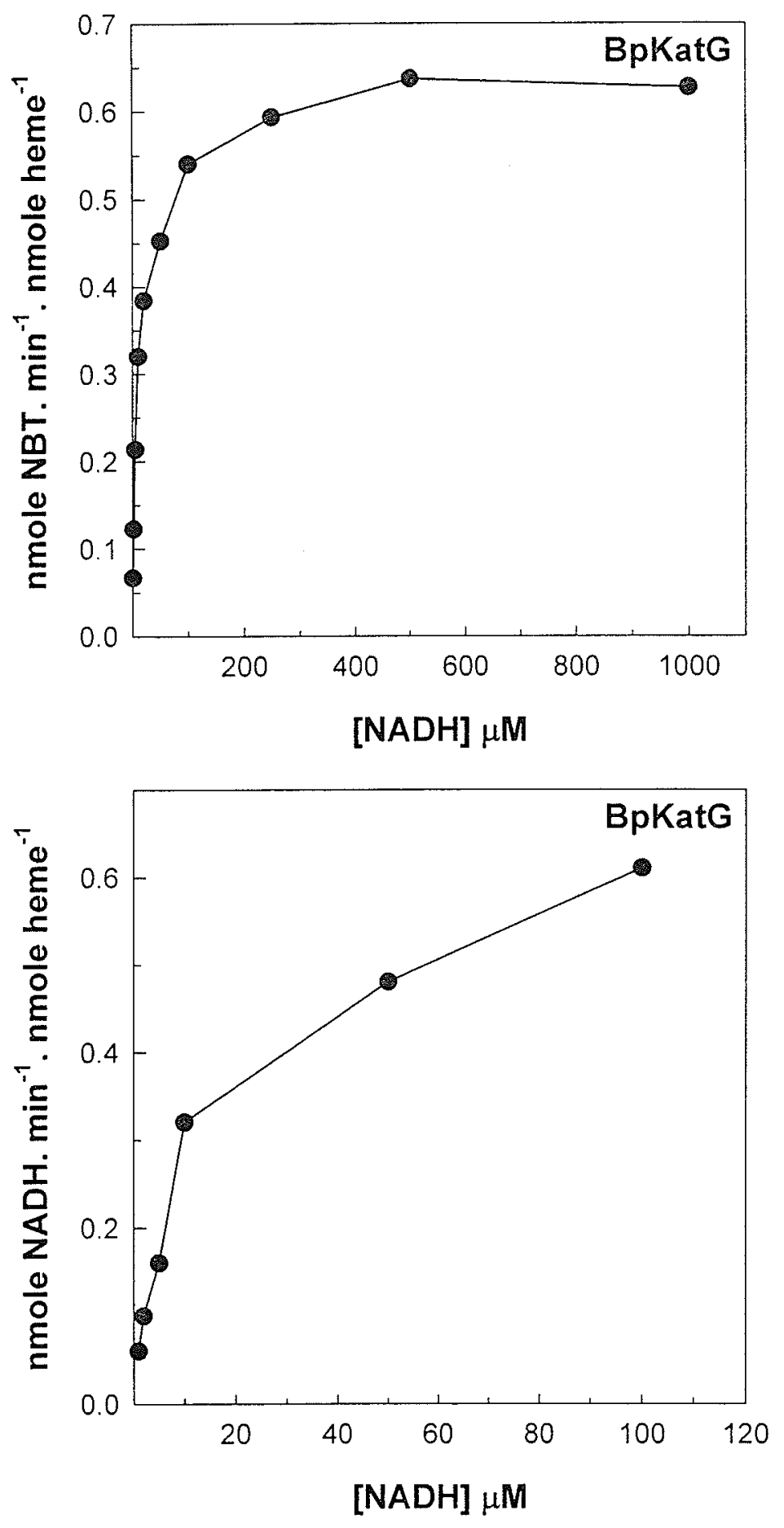

Figure 3.3.5. Effect of NADH concentration on the initial NADH oxidase velocities of $\mathrm{BpKatG}$ at $560 \mathrm{~nm}$ (top panel) and $340 \mathrm{~nm}$ (bottom panel). 

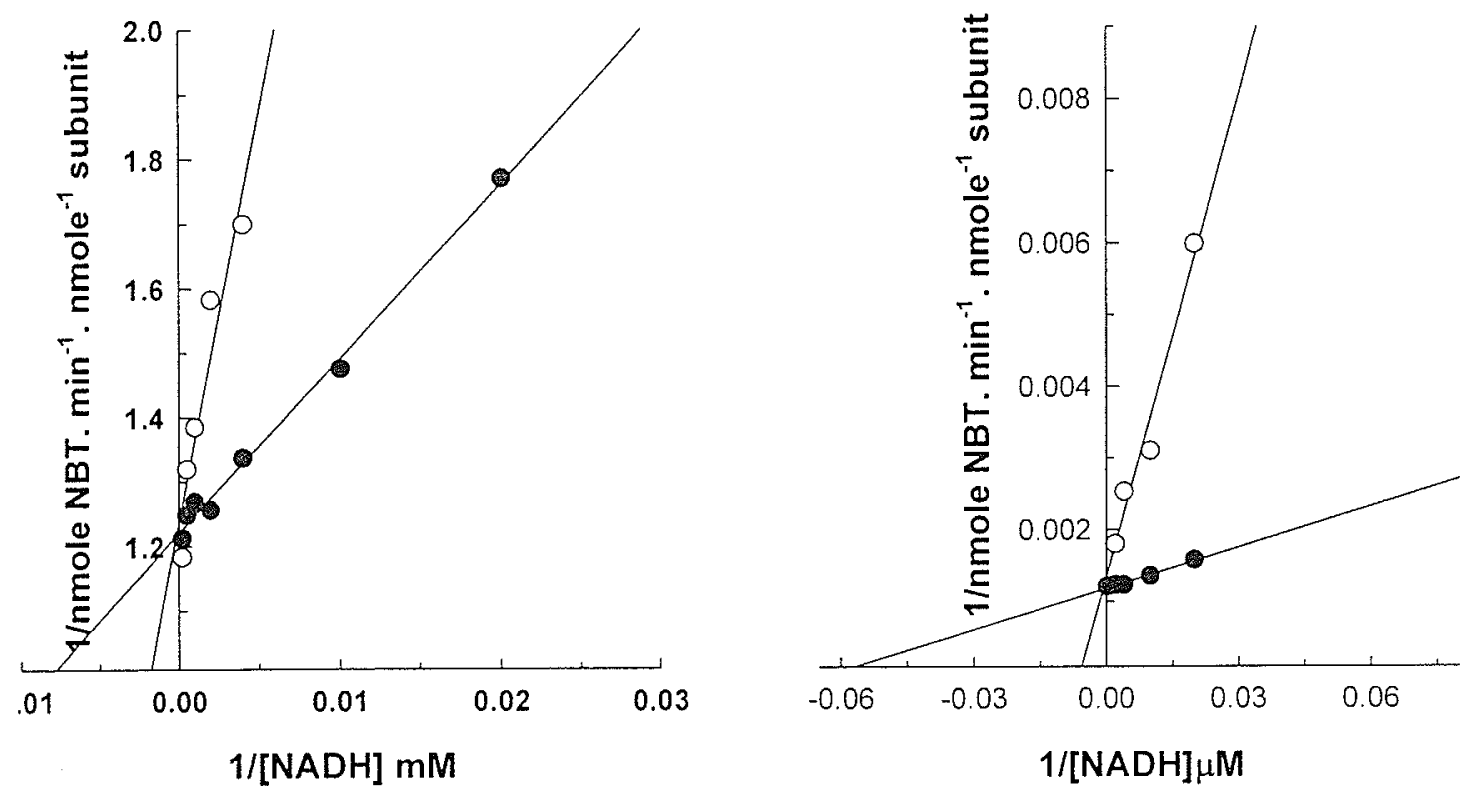

Figure 3.3.6. Effects of $\mathrm{NAD}^{+}$and pyridoxine on $\mathrm{NADH}$ oxidase activity. $1.2 \mu \mathrm{M}$ BpKatG was equilibrated with $5 \mathrm{mM} \mathrm{NAD}$ or pyridoxine before titrating with different concentrations of $\mathrm{NADH}$ at $\mathrm{pH}$ 8.75. Lineweaver-Burk plots (double reciprocal) are shown. 
Table 3.3.3. Comparison of kinetic constants for NADH oxidase, catalase and, peroxidase activities of $\mathrm{BpKatG}$

\begin{tabular}{lcccc}
\hline & $V_{\max }$ & $\boldsymbol{K}_{m}$ & $\boldsymbol{k}_{\text {cat }}$ & $\boldsymbol{k}_{\text {cat }} \boldsymbol{K}_{m}$ \\
\hline & $\mu m$ mole. $\mathrm{min}^{-1} \cdot \mu$ mole-heme & $\mu M^{-1}$ & $s^{-1}$ & $M^{l} \cdot s^{-1}$ \\
\hline Oxidase $\left(\Delta \mathbf{A}_{\mathbf{3 4 0}}\right)$ & $540 \pm 96 \times 10^{-3}$ & $12.5 \pm 2.0$ & $9.0 \pm 0.4 \times 10^{-3}$ & $7.2 \times 10^{2}$ \\
Peroxidase & $1083 \pm 29$ & $330 \pm 30$ & $10.1 \pm 0.27$ & $3.1 \times 10^{4}$ \\
Catalase & $902 \pm 20 \times 10^{3}$ & $7700 \pm 400$ & $8.4 \pm 0.2 \times 10^{3}$ & $1.1 \times 10^{6}$ \\
\hline
\end{tabular}

${ }^{a}[\mathrm{NADH}]$ for the oxidase reaction, [ABTS] for the peroxidase reaction, and $\left[\mathrm{H}_{2} \mathrm{O}_{2}\right]$ for the catalase reaction 
Table 3.3.4. Comparison of NADH oxidation by HRP, BpKatG, and the Trp 111 Phe variant. Reactions mixtures contained $100 \mu \mathrm{M} \mathrm{NADH}$, and $1.2 \mu \mathrm{M}$ either of $\mathrm{HRP}$, BpKatG or Trp 111 Phe in $50 \mathrm{mM}$ Tris, pH 8.75. $10 \mu \mathrm{M} \mathrm{H}_{2} \mathrm{O}_{2}, 300 \mathrm{nM}$ HPII or $6 \mu \mathrm{M}$ SOD was added as indicated. NADH oxidation was followed by decrease in absorbance at $340 \mathrm{~nm}$.

\begin{tabular}{lccc}
\hline & HRP & BpKatG & Trp111Phe \\
\hline $\mathbf{N A D H}$ & $110 \pm 18^{\mathrm{a}}$ & $490 \pm 16$ & $500 \pm 41$ \\
$\mathbf{N A D H}+\mathbf{H}_{2} \mathbf{O}_{2}$ & $360 \pm 22$ & $490 \pm 24$ & $2160 \pm 180$ \\
$\mathbf{N A D H}+\mathbf{H P I I}^{\mathrm{b}}$ & $30 \pm 4$ & $480 \pm 16$ & $392 \pm 16$ \\
$\mathbf{N A D H}+\mathbf{H P I I}+\mathbf{H}_{2} \mathbf{O}_{2}$ & $54 \pm 4$ & $480 \pm 16$ & $290 \pm 16$ \\
$\mathbf{N A D H}+\mathbf{S O D}^{\mathbf{c}}$ & $86 \pm 16$ & $400 \pm 24$ & $460 \pm 16$ \\
$\mathbf{N A D H}+\mathbf{S O D}+\mathbf{H}_{2} \mathbf{O}_{2}$ & $540 \pm 29$ & $400 \pm 16$ & $2200 \pm 190$ \\
\hline
\end{tabular}

${ }^{a}$ The data expressed as pmol NADH. min $^{-1}$ nmole $e^{-1}$ heme

${ }^{\mathrm{b}}$ Monofunctional catalase of E. coli

${ }^{\mathrm{c}}$ Superoxide dismutase 
Figure 3.3.5. Effect of $\mathrm{NADH}$ or $\mathrm{NAD}^{+}$on the peroxidase activity of $\mathrm{BpKatG}, \mathrm{MtKatG}$ and EckatG using ABTS or o-dianisidine as peroxidatic substrate.

\begin{tabular}{|c|c|c|c|c|c|c|}
\hline & \multicolumn{6}{|c|}{ ABTS } \\
\hline & \multicolumn{3}{|c|}{ Activity after $1 \mathrm{~min}(\mathrm{U} / \mathrm{mg})$} & \multicolumn{3}{|c|}{ Activity after $15 \mathrm{~min}(\mathrm{U} / \mathrm{mg})$} \\
\hline & BpKatG & MtkatG & EckatG & BpKatG & MtkatG & EckatG \\
\hline NO NADH/NAD ${ }^{+}$ & $3.8 \pm 0.1$ & $15 \pm 0.2$ & $12 \pm 0.4$ & $3.7 \pm 0.3$ & $15 \pm 0.3$ & $12 \pm 0.2$ \\
\hline NADH & $3.3 \pm 0.1$ & $15 \pm 0.2$ & $10 \pm 0.6$ & $2.9 \pm 0.1$ & $14 \pm 0.1$ & $11 \pm 0.4$ \\
\hline \multirow[t]{4}{*}{$\mathrm{NAD}^{+}$} & $4.9 \pm 0.1$ & $12 \pm 0.4$ & $10 \pm 0.3$ & $4.6 \pm 0.1$ & $14 \pm 0.6$ & $10 \pm 0.3$ \\
\hline & \multicolumn{6}{|c|}{$o$-dianisidine } \\
\hline & \multicolumn{3}{|c|}{ Activity after $1 \mathrm{~min}(\mathrm{U} / \mathrm{mg})$} & \multicolumn{3}{|c|}{ Activity after $15 \mathrm{~min}(\mathrm{U} / \mathrm{mg})$} \\
\hline & BpKatG & MtkatG & EckatG & BpKatG & MtkatG & EckatG \\
\hline NO NADH/NAD ${ }^{+}$ & $5.3 \pm 0.1$ & $10 \pm 0.2$ & $10 \pm 0.4$ & $5.3 \pm 0.3$ & $9.9 \pm 0.3$ & $10 \pm 0.2$ \\
\hline NADH & $5.4 \pm 0.1$ & $10 \pm 0.2$ & $9.9 \pm 0.6$ & $4.9 \pm 0.1$ & $10 \pm 0.1$ & $9.0 \pm 0.4$ \\
\hline $\mathrm{NAD}^{+}$ & $6.9 \pm 0.2$ & $11 \pm 0.4$ & $10 \pm 0.3$ & $6.7 \pm 0.1$ & $9.8 \pm 0.6$ & $9.7 \pm 0.3$ \\
\hline
\end{tabular}


comparison, NADH oxidation by the Trp 111 Phe variant, which lacks catalase activity, is reduced slightly by catalase, but not SOD, and is significantly enhanced by added $\mathrm{H}_{2} \mathrm{O}_{2}$, attributable to NADH serving as a peroxidatic substrate. No lag in the initiation of NADH oxidation was evident over a broad range of NADH concentrations or enzyme concentrations for either BpKatG or the Trp111Phe variant.

\subsubsection{Effect of NADH on the peroxidase activity of KatGs}

Effect of NADH and $\mathrm{NAD}^{+}$on the peroxidase activity of BpKatG, MtKatG and Eckat $\mathrm{G}$ was observed in order to determine the probability of $\mathrm{NADH}$ or $\mathrm{NAD}^{+}$having the similar binding site as that for the peroxidatic substrate such as ABTS or $o$-dianisidine in KatGs. A mixture of KatG equilibrated with $\mathrm{NADH}$ or $\mathrm{NAD}^{+}$was assayed at different time intervals for peroxidase activity using either ABTS or $o$-dianisidine as substrate. The results demonstrated only very subtle changes in peroxidase activity even after $15 \mathrm{~min}$ of incubation for both ABTS and o-dianisidine (Table 3.3.5), suggesting a separate binding site for $\mathrm{NADH} / \mathrm{NAD}^{+}$. Slight decrease in the peroxidase activity can be debated and the most plausible explanation could be the proximity between the binding sites for ABTS/odianisidine and $\mathrm{NADH} / \mathrm{NAD}^{+}$but this needs confirmation by structural analysis of the ligand-enzyme complex.

\subsubsection{INH hydrazine lyase activity by KatGs}

The activation of INH as an anti-tubercular drug by catalase-peroxidases involves removal of the hydrazine moiety and proceeds via a radical producing reaction that can be monitored using NBT as a radical sensor (Hillar and Loewen, 1995). The $\mathrm{H}_{2} \mathrm{O}_{2}$ 

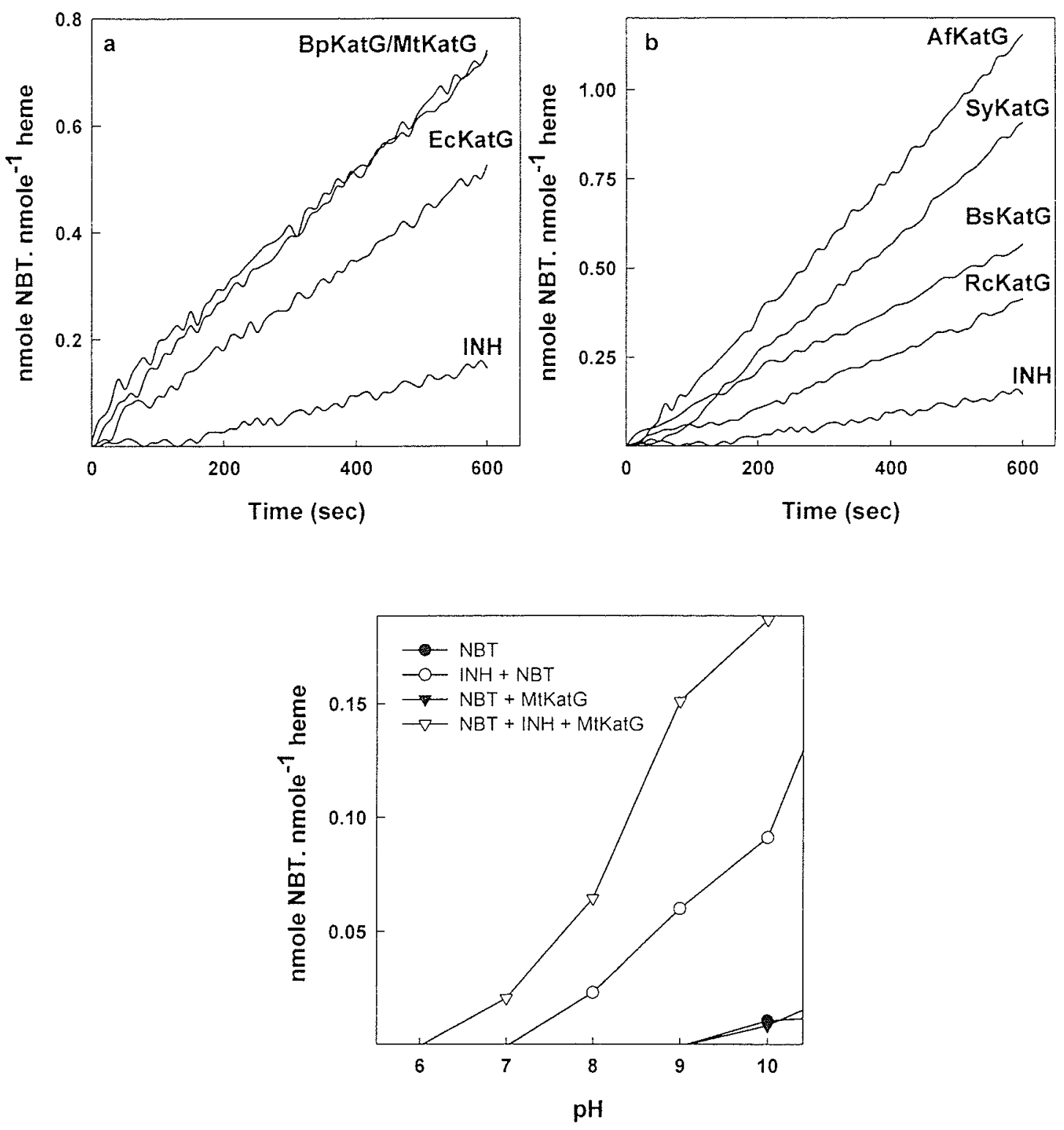

Figure 3.3.7. Removal of hydrazine form isoniazid. $a$ and $b$, Radical generation in a solution containing $10 \mathrm{mMINH}, 200 \mu \mathrm{M} \mathrm{NBT}$ as radical sensor in $50 \mathrm{mM}$ Tris, pH 8.0, was followed by formazan appearance measured at $560 \mathrm{~nm}$. Separate assays contain no enzyme (control), BpKatG, MtKatG, EcKatG, SyKatG, BskatG, AfKatG or RckatG. c, $\mathrm{pH}$ dependence of radical generation in a solution of $200 \mu \mathrm{M}$ NBT with and without MtKatG and a solution of $200 \mu \mathrm{M} \mathrm{NBT}$ and $10 \mu \mathrm{M} \mathrm{NNH}$ with and without MtKatG is shown. 
Figure 3.3.8. Effect of manganese on the removal of hydrazine lyase activity by KatG. $a$ and $b$, radical generation in a solution containing $10 \mathrm{mM} \mathrm{INH}, 2 \mu \mathrm{M} \mathrm{MnCl}_{2}$ and $200 \mu \mathrm{M}$ NBT in $50 \mathrm{mM}$ Tris, $\mathrm{pH} 8.0$, was followed by formazan appearance measured at $560 \mathrm{~nm}$. Separate assays contain no enzyme (control), BpKatG, MtKatG, EcKatG, SyKatG, BskatG, AfKatG or RckatG. 

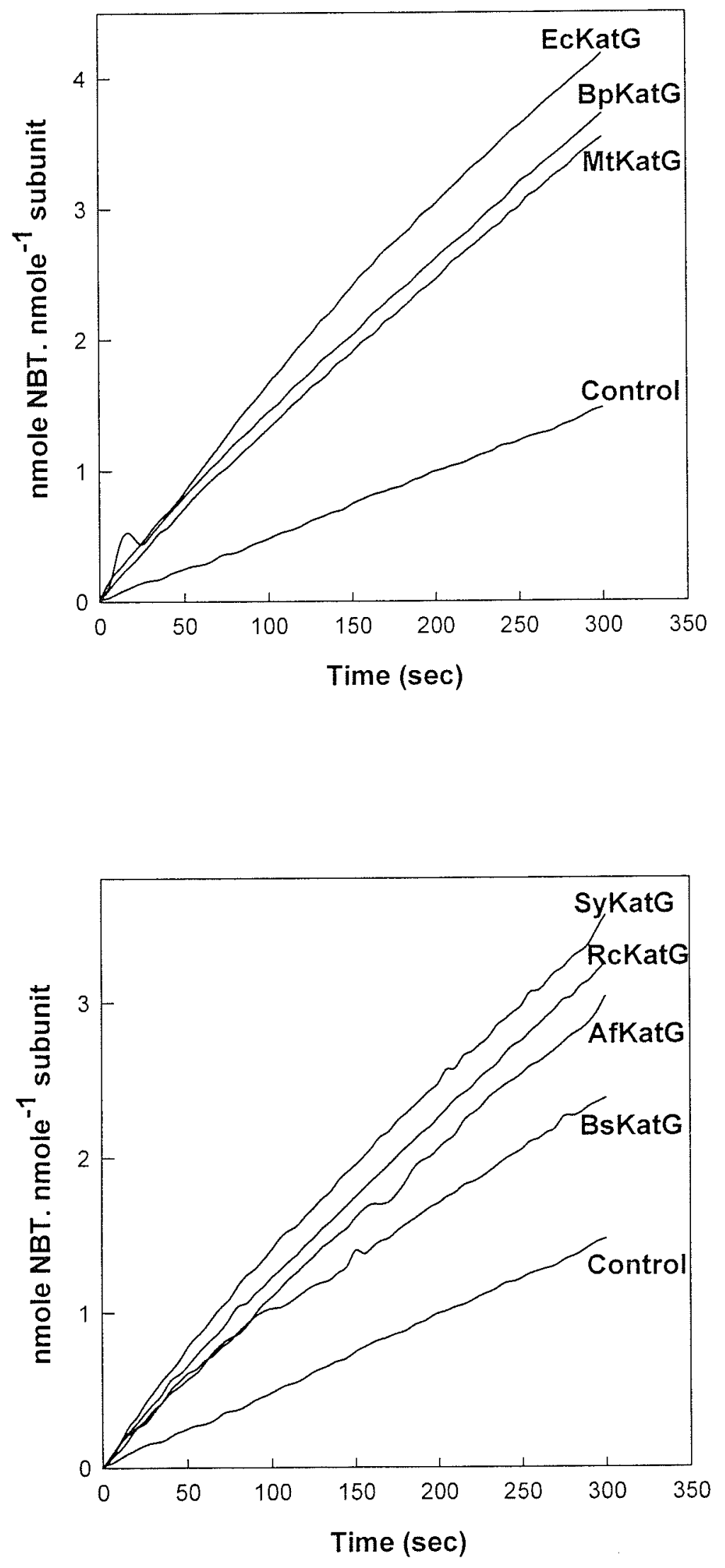
Table 3.3.6. Comparison of effect of $\mathrm{MnCl}_{2}$ and NADH on the hydrazine lyase activity of catalase-peroxidases.

\begin{tabular}{cccc}
\hline KatG & $\begin{array}{c}\text { Hydrazine lyase } \\
(\text { units } / \mathrm{mg})^{\mathrm{a}}\end{array}$ & $\begin{array}{c}\text { Hydrazine lyase } \\
\left(\text { with } 2 \mu \mathrm{M} \mathrm{Mn}^{2+}\right) \\
(\text { units/mg) }\end{array}$ & $\begin{array}{c}\text { INH-NADH } \\
\text { synergistic } \\
\text { reaction(units/mg) }\end{array}$ \\
\hline BpKatG & 76 & 410 & 1140 \\
MtKatG & 78 & 460 & 260 \\
EcKatG & 38 & 450 & 100 \\
SyKatG & 57 & 500 & 190 \\
BsKatG & 62 & 420 & 220 \\
AfkatG & 88 & 370 & 190 \\
RcKatG & 14 & 270 & 140 \\
\hline
\end{tabular}

${ }^{a}$ data expressed as pmole NBT. $\min ^{-1}$ nmole ${ }^{-1}$ heme. 
independence (Figures 3.3.7a \& b) and enhanced rates at alkaline pH (Figure 3.3.7c) of the KatG-mediated INH hydrazine lyase reaction differentiate it from the catalase and peroxidase reactions (Figure 3.3.3). There is a pH-dependent, nonenzymatic generation of radicals from $\mathbb{N H}$, but in the $\mathrm{pH} 7-9$ range, the contribution of the enzymatic reaction is clear and suggests a pH optimum between 8 and 9, albeit not well defined (Figure 3.3.7 c). To minimize the nonenzymatic contribution, all subsequent assays and reactions involving $\mathrm{NH}$ were carried out at $\mathrm{pH}$ 8. Oxygen levels do not affect the KatG-mediated radical generation from $\mathrm{INH}$, and superoxide dismutase does not reduce radical production (Table 3.3.2), confirming that molecular oxygen does not have a role in the reaction. AfKatG demonstrates the highest rate of hydrazine lyase whereas $\mathrm{MtKatG}$ shows almost the same rate as BpKatG, but the EcKatG-mediated reaction is slowest among the seven KatGs studied (Figure 3.3.7 and Table 3.3.6). Despite having a pyridine ring as part of its structure, $\mathrm{NAD}^{+}$does not inhibit $\mathrm{NH}$ hydrazine lyase (Table 3.3.2). Addition of $2 \mu \mathrm{M} \mathrm{Mn}^{2+}$ to a reaction mixture carrying INH and NBT resulted in a 4-5 fold increase in the radical production even in the absence of $\mathrm{KatG}$, but the addition of KatG significantly increased the rates as compared to the control lacking KatG (Figure 3.3.8 and Table 3.3.6).

\subsubsection{Interaction of $\mathrm{KatG}$ with a mixture of INH and NADH}

Radical generation in a mixture of $\mathrm{BpKatG}, \mathrm{NH}$, and $\mathrm{NADH}$ is about two times faster than the cumulative rate of the individual reactions of INH and NADH (Figures 3.3.9a and b). A similar pattern was observed for other KatGs which show significantly higher rates of radical formation as compared to the individual rates by 
Figure 3.3.9. Synergistic effect of $\mathrm{NH}$ and NADH on radical generation in a solution containing $10 \mathrm{mM} \mathrm{INH}, 100 \mu \mathrm{M}$ NADH, $200 \mu \mathrm{M}$ NBT as radical sensor in $50 \mathrm{mM}$ Tris, $\mathrm{pH} 8.0$, was followed by formazan appearance measured at $560 \mathrm{~nm}$. Separate assays contain, no enzyme (control), BpKatG, MtKatG, EcKatG, SyKatG, BskatG, AfKatG or RckatG. 

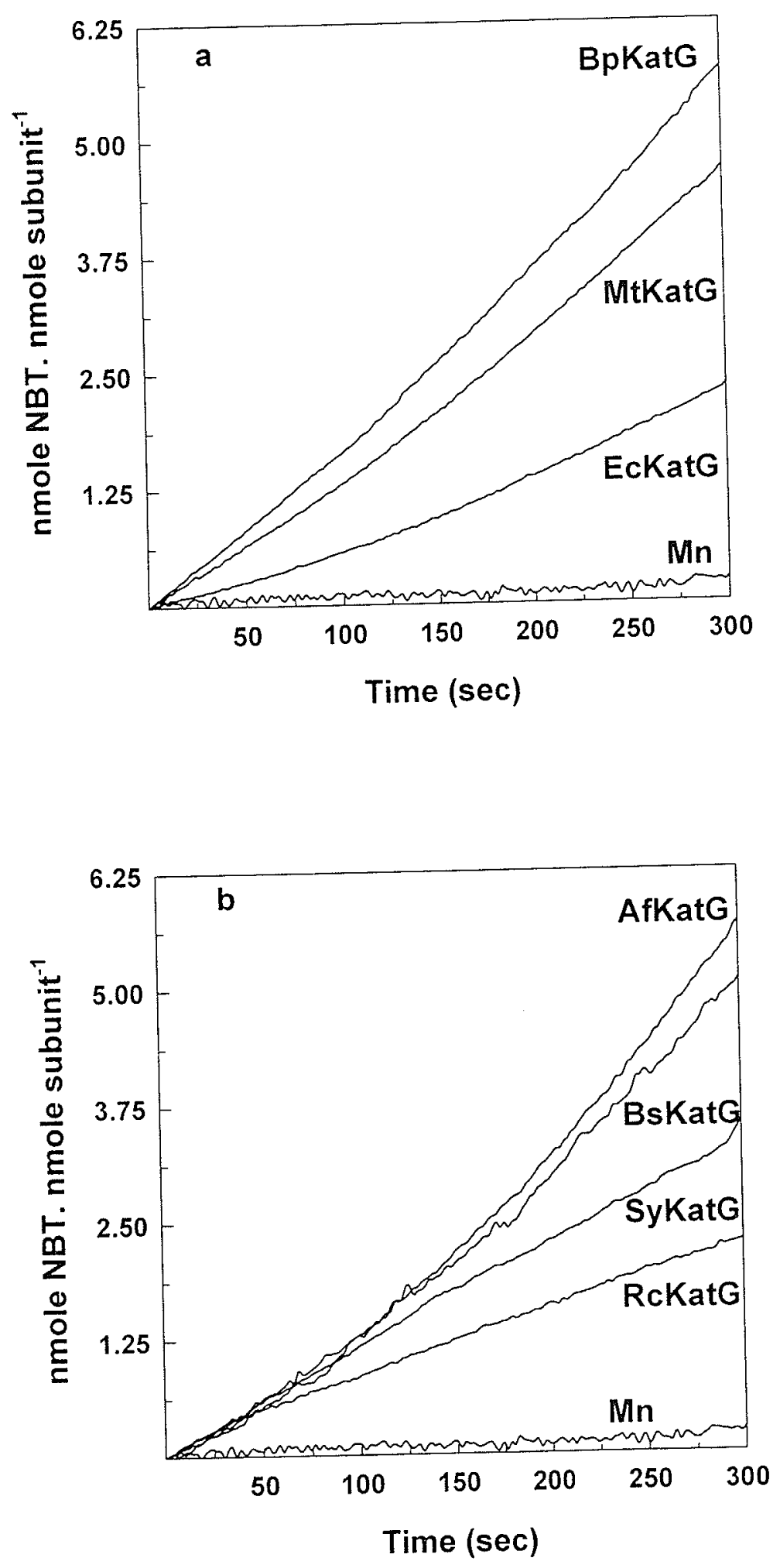
Figure 3.3.10. Elution profiles from reverse phase HPLC of reaction products from mixtures containing $100 \mu \mathrm{M} \mathrm{NADH}, 200 \mu \mathrm{M} \mathrm{NNH}$ and no enzyme (a); $1.2 \mu \mathrm{M} \mathrm{BpKatG}$ (b); $1.2 \mu \mathrm{M} \mathrm{MtKatG}$ (c); $1.2 \mu \mathrm{M} \mathrm{EcKatG}$ (d). I, isonicotinic acid hydrazide; $N-H$, $\mathrm{NADH} ; N-D, \mathrm{NAD}^{+} ; I-N$, isonicotinoyl-NAD. 


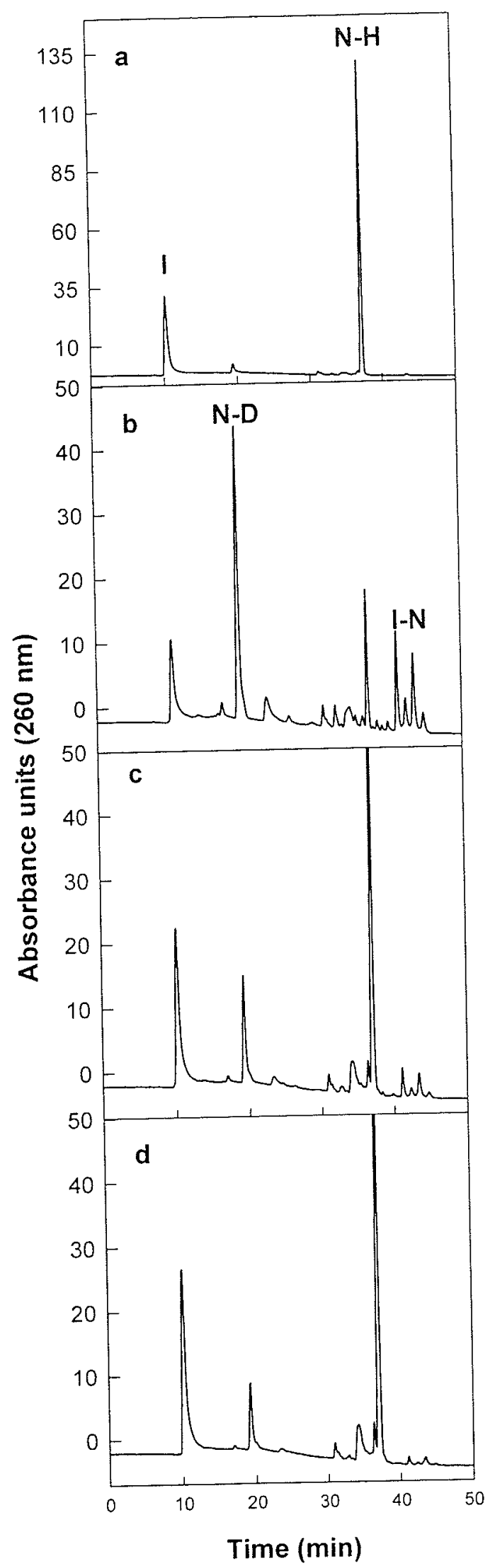


Figure 3.3.11. Elution profiles from reverse phase HPLC of reaction products from mixtures containing $100 \mathrm{mM} \mathrm{NADH}, 200 \mu \mathrm{M} \mathrm{INH}, 2 \mu \mathrm{M} \mathrm{MnCl}_{2}$ and no enzyme (a); 1.2 $\mu \mathrm{M} \mathrm{BpKatG}$ (b); $1.2 \mu \mathrm{M} \mathrm{MtKatG}$ (c); $1.2 \mu \mathrm{M} \mathrm{EcKatG}$ (d). I, isonicotinic acid hydrazide; $\mathrm{N}-\mathrm{D}, \mathrm{NAD}^{+} ; \mathrm{N}-\mathrm{H}, \mathrm{NADH} ; \mathrm{I}-\mathrm{N}$, isonicotinoyl-NAD. 


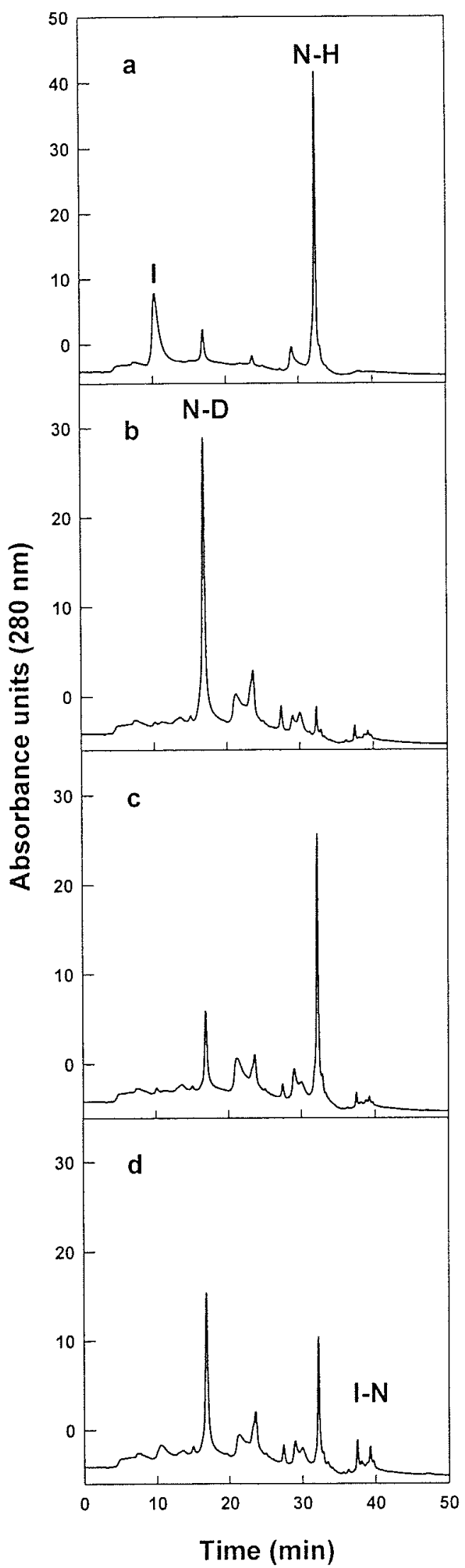




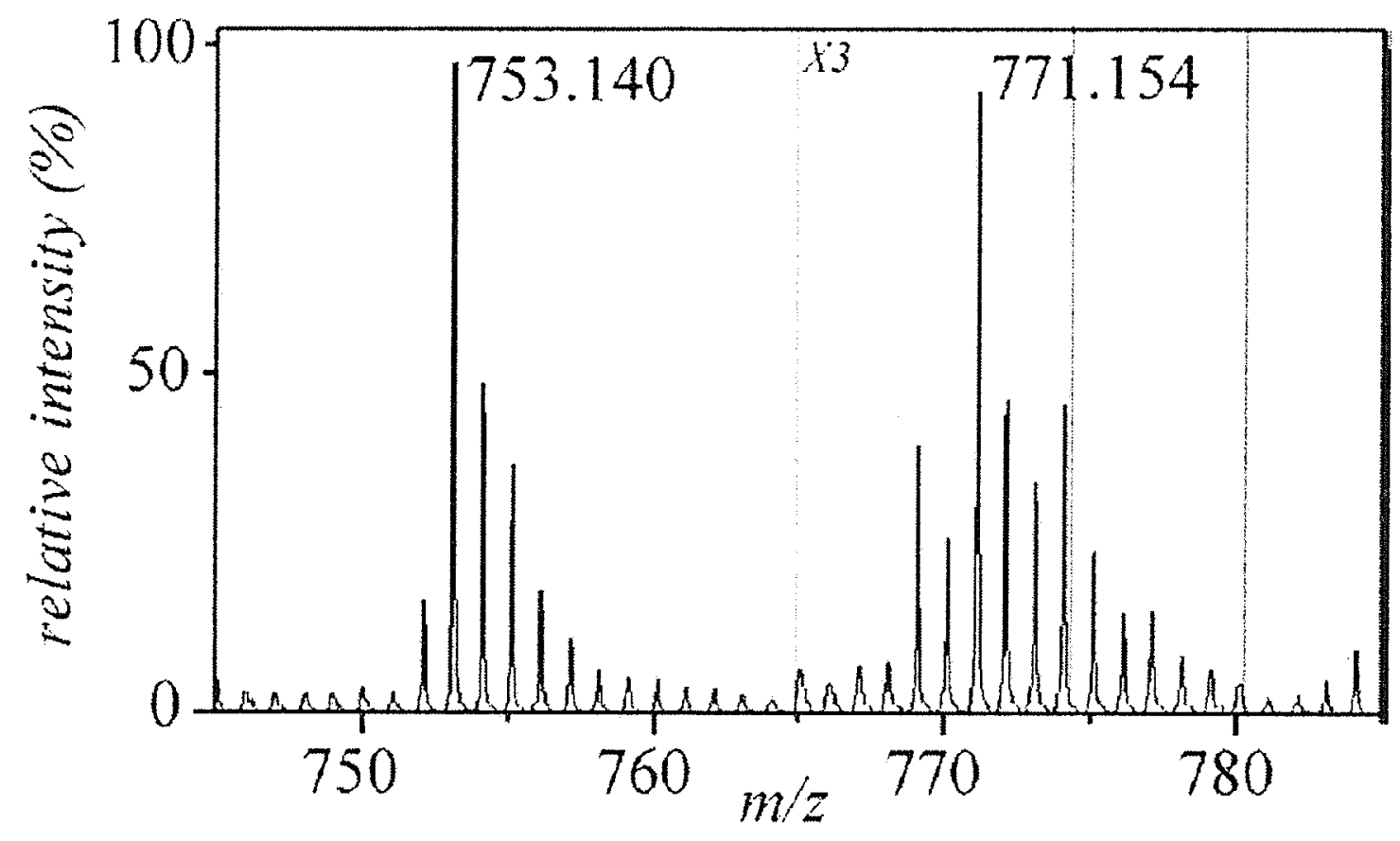

Figure 3.3.12. Part of a matrix-assisted laser desorption ionization mass spectrum of a reaction mixture after $18 \mathrm{~h}$ of incubation showing the isonicotinoyl-NAD product ion at $m / z 771$ and the decay product at $m / z$ 753. $N, \mathrm{NAD} ; I-N$, isonicotinoyl-NAD. 
Table 3.3.7. Comparison of expected and observed $\mathrm{m} / \mathrm{z}$ values of ions in the MS/MS spectra of NADH and the product of the reaction of KatG with INH and NADH identified as isonicotinoyl-NAD

\begin{tabular}{|c|c|c|c|c|c|c|}
\hline & \multicolumn{3}{|c|}{$\begin{array}{l}\text { Product ions }(m / z) \text { from } \\
\text { parent ion }{ }^{a}\end{array}$} & \multirow{2}{*}{$\begin{array}{l}\text { Assigned } \\
\text { structure }^{b}\end{array}$} & \multirow{2}{*}{$\begin{array}{l}\text { Formula to } \\
\text { calculate } \\
\text { expected ion }\end{array}$} & \multirow{2}{*}{$\begin{array}{c}\text { Expected } \\
m / z\end{array}$} \\
\hline & $m / z 771$ & $m / z 753$ & $m / z 666$ & & & \\
\hline \multirow[t]{3}{*}{ Parent ions } & 771.154 & & & IN-NRPPA & $\mathrm{C}_{27} \mathrm{H}_{33} \mathrm{~N}_{8} \mathrm{O}_{15} \mathrm{P}_{2}$ & 771.154 \\
\hline & 753.140 & 753.166 & & $\begin{array}{l}\text { IN-NRPPA- } \\
\quad \mathrm{H}_{2} \mathrm{O}\end{array}$ & $\mathrm{C}_{27} \mathrm{H}_{31} \mathrm{~N}_{8} \mathrm{O}_{14} \mathrm{P}_{2}$ & 753.144 \\
\hline & & & 666.090 & $\begin{array}{l}\text { NHRPPA } \\
\text { (NADH) }\end{array}$ & $\mathrm{C}_{21} \mathrm{H}_{30} \mathrm{~N}_{7} \mathrm{O}_{14} \mathrm{P}_{2}$ & 666.133 \\
\hline \multirow{5}{*}{$\begin{array}{l}\text { Fragmentation } \\
\text { from the adenine } \\
\text { end ("y" ions) }\end{array}$} & & & 530.061 & NHRPPR & $\mathrm{C}_{16} \mathrm{H}_{24} \mathrm{~N}_{2} \mathrm{O}_{14} \mathrm{P}_{2}$ & 530.070 \\
\hline & & & 514.059 & $\begin{array}{l}\text { NHRPPR- } \\
\mathrm{NH}_{3}\end{array}$ & $\mathrm{C}_{16} \mathrm{H}_{22} \mathrm{NO}_{14} \mathrm{P}_{2}$ & 514.052 \\
\hline & & 424.101 & 319.073 & $\begin{array}{l}\text { IN-NRP } \\
\text { NHRP }\end{array}$ & $\begin{array}{l}\mathrm{C}_{17} \mathrm{H}_{19} \mathrm{~N}_{3} \mathrm{O}_{8} \mathrm{P} \\
\mathrm{C}_{11} \mathrm{H}_{16} \mathrm{~N}_{2} \mathrm{O}_{7} \mathrm{P}\end{array}$ & $\begin{array}{l}424.091 \\
319.070\end{array}$ \\
\hline & 229.044 & & & $\mathrm{IN}-\mathrm{N}$ & $\mathrm{C}_{12} \mathrm{H}_{10} \mathrm{~N}_{3} \mathrm{O}_{2}$ & 229.085 \\
\hline & & & 123.063 & $\mathrm{NH}$ & $\mathrm{C}_{6} \mathrm{H}_{7} \mathrm{~N}_{2} \mathrm{O}$ & 123.056 \\
\hline \multirow[t]{8}{*}{$\begin{array}{l}\text { Fragmentation } \\
\text { from the } \\
\text { nicotinamide end } \\
\text { ("b" ions) }\end{array}$} & & & 649.109 & $\begin{array}{l}\text { NHRPPA- } \\
\mathrm{NH}_{3}\end{array}$ & $\mathrm{C}_{2 \mathrm{I}} \mathrm{H}_{27} \mathrm{~N}_{6} \mathrm{O}_{14} \mathrm{P}_{2}$ & 649.106 \\
\hline & 664.126 & & & NRPPA & $\mathrm{C}_{21} \mathrm{H}_{28} \mathrm{~N}_{7} \mathrm{O}_{14} \mathrm{P}_{2}$ & 664.117 \\
\hline & 542.078 & 542.072 & 542.082 & RPPA & $\mathrm{C}_{15} \mathrm{H}_{22} \mathrm{~N}_{5} \mathrm{O}_{13} \mathrm{P}_{2}$ & 542.069 \\
\hline & 524.059 & 524.067 & 524.067 & RPPA- $\mathrm{H}_{2} \mathrm{O}$ & $\mathrm{C}_{15} \mathrm{H}_{20} \mathrm{~N}_{5} \mathrm{O}_{12} \mathrm{P}_{2}$ & 524.058 \\
\hline & 428.041 & 428.023 & 428.040 & PPA & $\mathrm{C}_{10} \mathrm{H}_{16} \mathrm{~N}_{5} \mathrm{O}_{10} \mathrm{P}_{2}$ & 428.037 \\
\hline & 348.073 & 348.070 & 348.071 & $\mathrm{PA}$ & $\mathrm{C}_{10} \mathrm{H}_{15} \mathrm{~N}_{5} \mathrm{O}_{7} \mathrm{P}$ & 348.071 \\
\hline & 250.094 & 250.087 & 250.100 & A & $\mathrm{C}_{10} \mathrm{H}_{12} \mathrm{~N}_{5} \mathrm{O}_{3}$ & 250.094 \\
\hline & 136.070 & 136.065 & 136.064 & Adenine & $\mathrm{C}_{5} \mathrm{H}_{6} \mathrm{~N}_{5}$ & 136.062 \\
\hline
\end{tabular}

${ }^{a}$ The parent ions at $\mathrm{m} / \mathrm{z} 771.153,753.166$, and 666.090 were selected individually and fragmented by tandem mass spectrometry.

${ }^{\mathrm{b}} \mathrm{A}$, adenosine; $\mathrm{N}$, nicotinamide; $\mathrm{IN}$, isonicotinoyl group; $\mathrm{R}$, ribose 
using NADH or INH as substrate (Figure 3.3.9a and $\mathrm{b}$ and Table 3.3.6). This rate is further enhanced by the inclusion of $2 \mu \mathrm{M}$ manganese ion (either $\mathrm{Mn}^{2+}$ or $\mathrm{Mn}^{3+}$ ) (Table 3.3.2). $\mathrm{NAD}^{+}$is not a substrate for radical generation, and the rate of radical formation from a mixture of $\mathrm{NAD}^{+}$and INH is the same as with INH alone (Table 3.3.2) and is enhanced by $\mathrm{Mn}^{2+}$ (Table 3.3.2). The MtKatG and EcKatG mediated reactions of INH and NADH are both slower in the absence of $\mathrm{Mn}^{2+}$ (Table 3.3.2) but approach or exceed the BpKatG-mediated reaction in the presence of $\mathrm{Mn}^{2+}$ (Table 3.3.2). Based on HPLC retention times (Figure 3.3.10) combined with mass spectrometry and tandem mass spectrometry analysis (Table 3.3.9), the main products of the combined NADH and INH reaction with $\mathrm{KatG}$ are $\mathrm{NAD}^{+}$and isonicotinoyl-NAD with other products being present in smaller amounts. Isonicotinoyl-NAD elutes from the column as four peaks, all exhibiting an ion at $m / z 771$, consistent with a previous report (Wilming and Johnsson, 1999), which explained the elution pattern as the result of two stereoisomers, arising from the addition of the isonicotinoyl group to opposite faces of the nicotinamide ring, each with two rotamers, resulting from restricted rotation of the isonicotinoyl group. The time course of appearance and identity of isonicotinoyl-NAD were confirmed by mass spectrometry analysis of the reaction mixture, and the product ion at $\mathrm{m} / \mathrm{z} 771$, coincident with the mass of isonicotinoyl-NAD, was evident only in mixtures containing all three of $\mathrm{KatG}, \mathbb{N N H}$, and $\mathrm{NADH}$ or $\mathrm{NAD}^{+}$(discussed in the next section). Tandem mass spectrometry measurements of the ions at $\mathrm{m} / \mathrm{z} 753$ and 771 produced almost identical fragmentation patterns, confirming that the ion at $m / z 753$ is a product of the parent ion at $m / z 771$ (Table 3.3.9). The difference in mass between the two ions, $18.014 \mathrm{Da}$, suggests the loss of $\mathrm{H}_{2} \mathrm{O}(18.016 \mathrm{Da})$ but offers no clue as to the structural basis for the decay or 
its cause. Comparison with the fragmentation pattern of NADH clearly confirms the presence of NAD in the pattern of fragments from the $m / z 771$ and 753 ions (Figure 3.3.12 and Table 3.3.9) degraded from the nicotinamide end and by inference allowing for the isonicotinoyl group, also after degradation from the adenine end (Table 3.3.9).

\subsubsection{1. $\mathrm{NAD}^{+}$as Precursor for Isonicotinoyl-NAD}

The facile KatG-mediated oxidation of NADH and lack of apparent reaction with $\mathrm{NAD}^{+}$initially suggested that $\mathrm{NADH}$, not $\mathrm{NAD}^{+}$, is the precursor to isonicotinoyl-NAD. However, isonicotinoyl-NAD is formed in a KatG-mediated reaction more efficiently from a mixture of $\mathrm{NAD}^{+}$and INH (Figure 3.3.14) than from a mixture of NADH and $\mathrm{INH}$, both with $2 \mu \mathrm{M} \mathrm{Mn}^{2+}$ (Figure 3.3.11). Therefore, $\mathrm{NAD}^{+}$is the probable precursor for isonicotinoyl-NAD as concluded earlier (Wilming and Johnsson, 1999), but NADH oxidation by $\mathrm{KatG}$ to $\mathrm{NAD}^{+}$will lead to the same product in an $\mathrm{NADH}$ mixture. The presence of $2 \mu \mathrm{M} \mathrm{Mn}^{2+}$ greatly speeds the $\mathrm{NAD}^{+}$-dependent formation of isonicotinoylNAD by all seven KatGs: BpKatG, MtKatG, EcKatG, SyKatG, BsKatG, AfKatG and RcKatG (Table 3.3.7).

\subsubsection{Comparison of the rate of isonicotinoyl-NAD synthase activity by KatGs}

The synthesis of the isonicotinoyl-NAD adduct was monitored at $326 \mathrm{~nm}$ and the rate was determined using an extinction coefficient of $6900 \mathrm{M}^{-1} \mathrm{~cm}^{-1}$ (Rawat et al., 2003). All seven KatGs shows substantially higher rates for the synthase activity as compared to the control containing $\mathbb{N H}, \mathrm{NAD}^{+}$, and $\mathrm{Mn}^{2+}$ but no KatG (Figure 3.3.13 and Table 3.3.7) clearly demonstrating the significant role of KatGs in the formation of 
Figure 3.3.13. Elution profiles from reverse phase HPLC of reaction products from mixtures containing $100 \mu \mathrm{M} \mathrm{NAD}^{+}, 200 \mu \mathrm{M} \mathrm{NNH}, 2 \mu \mathrm{M} \mathrm{MnCl}_{2}$ and no enzyme (a); 1.2 $\mu \mathrm{M}$ BpKatG (b); $1.2 \mu \mathrm{M}$ MtKatG (c); $1.2 \mu \mathrm{M} \mathrm{EcKatG}(\mathrm{d}) . I$, isonicotinic acid hydrazide; $N-D, \mathrm{NAD}^{+} ; I-N$. isonicotinoyl-NAD. $l_{2}(\mathrm{~d}) . I$, 


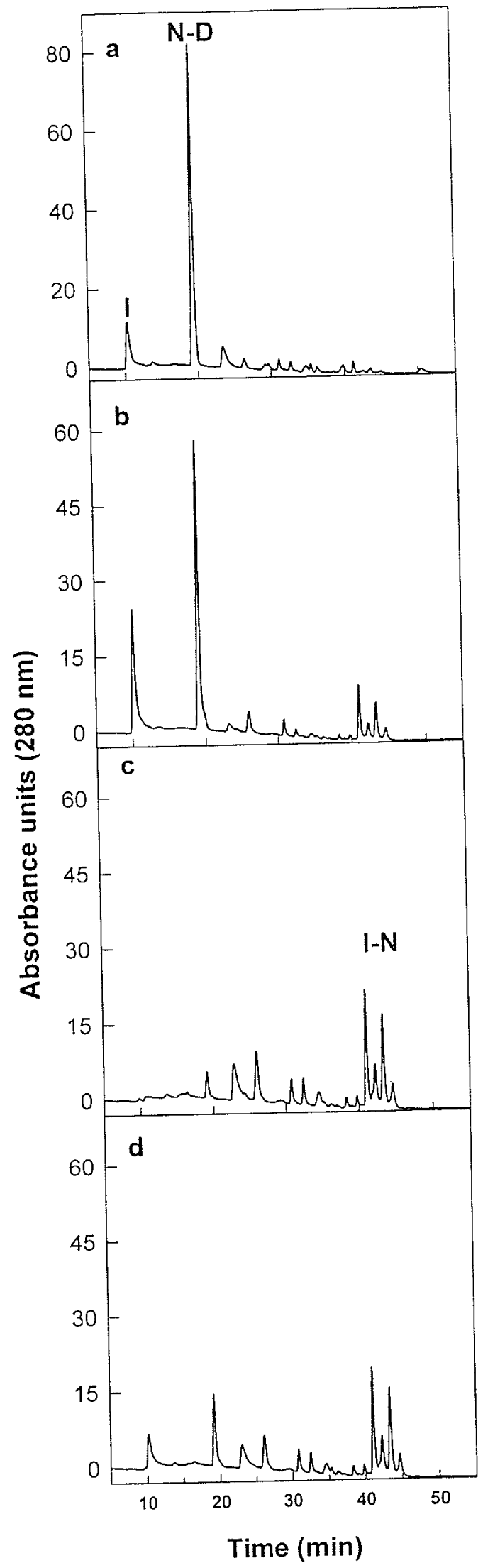


Figure 3.3.14. Rate of synthesis of the isonicotinoyl-NAD adduct, $a$ and $b$. The reaction mixture contained $100 \mu \mathrm{M} \mathrm{NAD}{ }^{+}, 200 \mu \mathrm{M} \mathrm{NNH}, 2 \mu \mathrm{M} \mathrm{MnCl}_{2}$, in $50 \mathrm{mM}$ Tris-HCL (pH 8.0). Separate assays contain, no KatG, or $1.2 \mu \mathrm{M}$ of BpKatG, MtKatG, EcKatG, SyKatG, BsKatG, AfKatG, RcKatG. Absorbance at $326 \mathrm{~nm}$ was monitored and an extinction coefficient of $6900 \mathrm{M}^{-1} \mathrm{~cm}^{-1}$ was used to calculate the amount of isonicotinoylNAD formed, assuming no interference from other minor products formed in the reaction. 

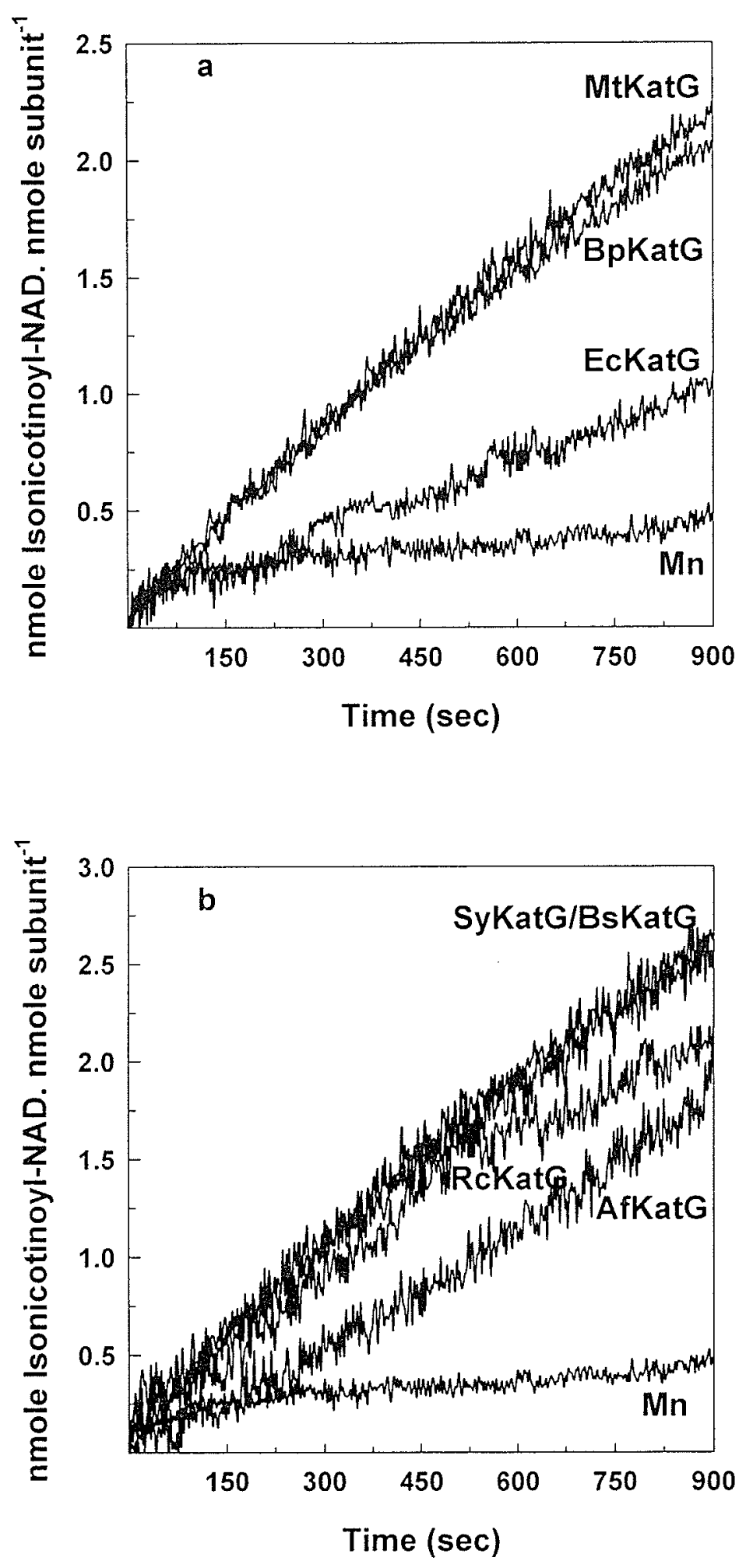
isonicotinoyl-NAD adduct. Among the seven KatGs compared in this study, SyKatG demonstrates the highest rate for synthase activity followed by BsKatG, RcKatG, MtKatG, BpKatG, AfKatG and the EcKatG being the lowest.

\subsubsection{NADH Oxidation Can Be Uncoupled from Superoxide Formation}

It has been well documented that changing any one of a number of residues in KatG reduces catalase activity with minimal effect on peroxidase activity (Jakopitsch et al., 2003; Yu et al., 2003, Jakopitsch et al., 2003; Regelsberger et al, 2000; Hillar et al., 2000). A number of variants of BpKatG with residues changed in the active site cavity and in the Met-Tyr-Trp cross-linked structure (Yamada et al., 2002; Carpena et al., 2003) were assayed for oxidase and hydrazine lyase activities for comparison with their catalase and peroxidase activities (Table 3.6.8). The hydrazine lyase reaction was largely unaffected by any of the changes except the change of His112 to Ala. By contrast, the oxidase reaction was affected in several of the variants, and two groups with different properties can be discerned. One group, including those with changes in any of the three residues in the cross-linked structure of Trp111, Tyr238, and Met264, exhibits normal rates of NADH disappearance but significantly reduced rates of radical production. The apparent uncoupling of $\mathrm{NADH}$ oxidation from superoxide generation could be a result of a broken electron tunnel or of a change in the reaction chemistry to favor $\mathrm{H}_{2} \mathrm{O}_{2}$ over superoxide formation. Unfortunately, the unambiguous identification or quantification of $\mathrm{H}_{2} \mathrm{O}_{2}$ formed in the reaction is not possible because it is immediately utilized in a peroxidatic reaction. Indeed, an inconclusive 13\% hypochromic and 3-5-nm red shift in the Soret band and little change in the 500-700 $\mathrm{nm}$ region are observed for the 
Table 3.3.8. Comparison of catalase, peroxidase, and oxidase activities of BpKatG and its variants.

\begin{tabular}{|c|c|c|c|c|c|}
\hline & Catalase & Peroxidase & Oxidase $_{(340 \mathrm{~nm})}$ & Oxidase $_{(560 \mathrm{~nm})}$ & $\begin{array}{c}\text { Hydrazine } \\
\text { lyase }\end{array}$ \\
\hline & \multicolumn{5}{|c|}{ units/mg } \\
\hline BpKatG & $4100 \pm 200$ & $4.4 \pm 0.5$ & $6.9 \pm 1.0$ & $7.7 \pm 1.2$ & $0.9 \pm 0.02$ \\
\hline Arg108Ala & $1250 \pm 110$ & $1.0 \pm 0.1$ & $3.7 \pm 0.5$ & $2.2 \pm 0.4$ & $0.3 \pm 0.01$ \\
\hline Arg108Lys & $320 \pm 20$ & $09 \pm 0.1$ & $2.2 \pm 0.2$ & $1.3 \pm 0.1$ & $0.32 \pm 0.01$ \\
\hline His 112Ala & $1 \pm 0.2$ & $0.1 \pm 0.01$ & $N^{a}$ & $N^{a}$ & $\mathrm{ND}^{\mathrm{a}}$ \\
\hline His112Asn & $2 \pm 0.6$ & $0.1 \pm 0.01$ & $3.1 \pm 0.2$ & $1.3 \pm 0.5$ & $0.2 \pm 0.06$ \\
\hline Trp111Phe & $2 \pm 0.1$ & $3.3 \pm 0.6$ & $7.7 \pm 2.4$ & $N^{a}$ & $0.5 \pm 0.04$ \\
\hline Asp141Ala & $60 \pm 10$ & $5.8 \pm 0.4$ & $2.2 \pm 0.1$ & $0.7 \pm 0.1$ & $0.5 \pm 0.1$ \\
\hline Asp141Asn & $390 \pm 20$ & $5.4 \pm 0.3$ & $2.8 \pm 0.2$ & $1.2 \pm 0.2$ & $0.6 \pm 0.1$ \\
\hline Asp141Glu & $3290 \pm 40$ & $6.3 \pm 1.7$ & $3.4 \pm 0.1$ & $2.8 \pm 0.2$ & $0.8 \pm 0.02$ \\
\hline Tyr238Ala & $2 \pm 0.1$ & $6.1 \pm 0.1$ & $10.8 \pm 2.0$ & $0.4 \pm 0.1$ & $0.5 \pm 0.04$ \\
\hline Tyr238Phe & $6 \pm 1.1$ & $2.8 \pm 0.3$ & $10.3 \pm 2.0$ & $N^{a}$ & $1.1 \pm 0.05$ \\
\hline Met264Ala & $6 \pm 1.1$ & $7.0 \pm 1.4$ & $4.3 \pm 0.1$ & $\mathrm{ND}^{\mathrm{a}}$ & $0.6 \pm 0.04$ \\
\hline Met264Leu & $2 \pm 0.9$ & $6.1 \pm 1.3$ & $11.0 \pm 1.5$ & 4.90 .6 & $1.0 \pm 0.06$ \\
\hline MtKatG & $3550 \pm 160$ & $9.7 \pm 0.2$ & $2.4 \pm 0.8$ & $1.2 \pm 0.2$ & $1.0 \pm 0.06$ \\
\hline EcKatG & $2320 \pm 230$ & $9.9 \pm 0.3$ & $1.1 \pm 0.4$ & $0.2 \pm 0.05$ & $0.5 \pm 0.02$ \\
\hline
\end{tabular}

${ }^{a} \mathrm{ND}$, not determined.

BpKatG variants Arg 108Ala, Arg108Lys, His112Ala, His112Asn, Asp 141 Ala, Asp 141Asn, Asp141Glu were constructed and characterized by MR. Taweewat Deemagarn; Tyr238Ala, Tyr238Phe, Met264Ala, Met264Lys were constructed and characterized by MR. Ben Wiseman; Trp 111 Phe was constructed by MR. Jack Switala 
Trp111Phe and Tyr238Phe variants during the oxidase reaction, whereas no change in native BpKatG. The second group exhibits reduced rates of both NADH disappearance and superoxide formation, consistent with reduced NADH oxidation. This group includes those with changes in the active site residues Arg108, His112, and Asp141. The changes in catalase and peroxidase activities caused by the sequence changes are consistent with those reported previously for KatGs from Synechocystis (Regelsberger et al, 2000; Yu et al, 2003; Regelsberger et al, 2000), M. tuberculosis (Yu et al, 2003), and E. coli (Hillar et al., 2000).

\subsection{Resistance against isoniazid in Mycobacterium tuberculosis.}

\subsubsection{Introduction}

Multidrug-resistant strains of Mycobacterium tuberculosis seriously threaten tuberculosis (TB) control and prevention efforts. Resistance against isoniazid was reported soon after its first use in 1951 (Middlebrook, 1954). Simultaneously a correlation between INH resistance and the loss of catalase-peroxidase activity was discovered in the early 1950's from the clinical isolates of M. tuberculosis (Middlebrook et al., 1954a; Middlebrook, 1954b; Middlebrook, 1954c). There was also a report mentioning kat $G$ deletion from the chromosome of two strains with high level INHresistance isolates (MIC $>50 \mathrm{mg} / \mathrm{ml}$ ) (Zhang et al., 1992). However, many subsequent studies have shown that the vast majority of $\mathrm{INH}$ resistance strains contain the $k a t G$ gene, a result indicating that deletion of this gene is very rare among patient isolates (Ramaswamy and Musser, 1998 and other reviews therein). Almost 50-60\% INH 
resistance patient isolates have missense mutations, or small deletions or insertions that are not represented among INH sensitive control strains (Musser, 1995). A wide array of distinct KatG mutations have been reported among INH resistance isolates but a point mutation of Ser at position 315 is the most abundant. Statistically, the most common amino acid substitution is AGC (Ser)->ACC (Thr), but ACA (Thr), ATC (Ile),AGA (Arg), CGC (Arg), AAC (Asp), and GGC (Gly) changes also have been identified (Ramaswamy and Musser, 1998). As a result, Ser315Thr is one of the most characterized variants of $\mathrm{MtKatG}$ and has recently had its crystal structure determined (Zhao et al., 2006). To determine the effect of Ser315 mutation on hydrazine lyase, NADH oxidase and isonicotinoyl-NAD synthase activities of KatGs, Ser315Thr and Ser315Asn variants of MtKatG were constructed and characterized.

\subsubsection{Purification of Ser315Thr, Ser315Asn variants of $M$. tuberculosis catalase- peroxidase}

Purification of Ser315Thr and S315Asn variants employed the same protocol as described in section 3.2.1. Purified variants migrate on denaturing SDS-PAGE predominantly as a single band with a molecular mass of $\sim 80 \mathrm{kDa}$ (Figure 3.4.1). Comparative absorption spectra of the native $\mathrm{Mtkat} G$ and the variants revealed similar absorbance peaks in the Sorêt region at $408 \mathrm{~nm}$ and in the charge transfer region (Figure 3.4.2 and Table 3.4.1). The Rz ratio of both Ser315Thr and Ser315Ans was comparable to that of native enzyme (Table 3.4.1) demonstrating more than $90 \%$ occupancy of heme. 


\subsubsection{Catalase and peroxidase specific activities of MtKatG and variants}

Purified Ser315Thr variant exhibited catalase specific activity comparable to that of the native enzyme, while the Ser315Asn variant exhibited a $50 \%$ decrease in the catalase specific activity (Table 3.4.2). The peroxidase specific activities of the variants are significantly lower than those of the native enzyme with Ser $315 \mathrm{Thr}$ being only $50 \%$ and $60 \%$ as active with ABTS and $o$-dianisidine respectively as electron donors. Even more striking, Ser315Asn variant is only $10 \%$ and $35 \%$ as active as the native enzyme with ABTS and o-dianisidine, respectively (Table 3.4.2).

\subsubsection{Kinetic characterization of Ser315Thr and Ser315Asn variants.}

A comparative analysis of the catalatic kinetic constants for native $\mathrm{MtKatG}$ and variants has been demonstrated in the Table 3.4.3 and Figure 3.4.3. They reveal comparable $V_{\max }$ values for both the MtKatG and Ser315Thr variants with Ser315Asn about $50 \%$ lower. Surprisingly the $K_{\mathrm{m}}$ of the Ser315Thr variant for $\mathrm{H}_{2} \mathrm{O}_{2}$ is six times

higher than that of the native enzyme whereas the $K_{\mathrm{m}}$ of the Ser315Asn variant for $\mathrm{H}_{2} \mathrm{O}_{2}$ is lower by $60 \%$ than the native enzyme. The changes in the $V_{\max }$ and $K_{\mathrm{m}}$ values are indicative of the changes caused by these mutations in the heme environment and disruption in the substrate access channel to the active site heme. Ser315Thr consistent with the specific activities, exhibits a turnover rate comparable to that of native enzyme with a much reduced turnover rate, whereas Ser315Asn is reduced to almost $50 \%$ but with a specificity constant similar to that of the native enzyme.

Peroxidatic kinetic constants for native MtKatG and Ser315Thr and Ser315Asn variants are shown in the Table 3.4.4 and Figures 3.4.5 and 3.4.6. Both variants exhibit a 

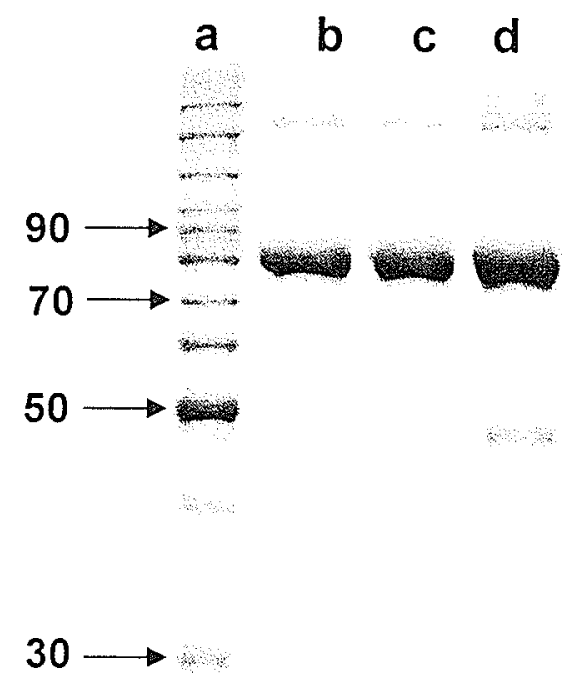

mo

Figure 3.4.1. SDS-polyacrylamide analysis of purified MtKatG and variants Ser315Thr and Ser315Asn. Approxmately $5 \mu \mathrm{g}$ of protein was loaded and run on an $8 \%$ polyacrylamide gel and stained with Coomassie brilliant blue. Lane $a$, protein ladder; $b$ MtKatG; $c$, Ser315Thr; d, Ser315Asn. 

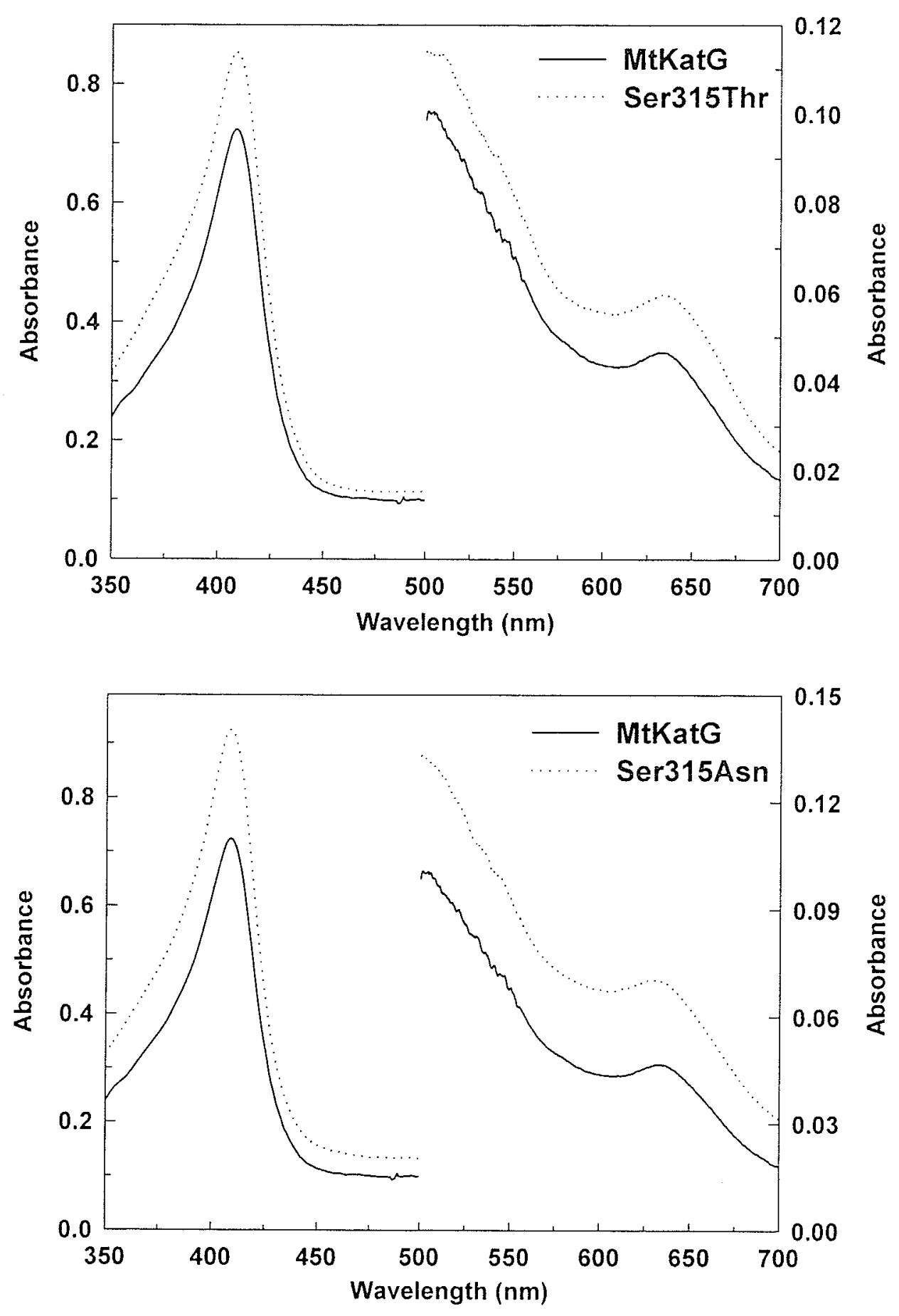

Figure 3.4.2. Absorption spectra of MtKatG and variants Ser315Thr and Ser315Asn. The left axis is for the range from 350 to $500 \mathrm{~nm}$ while the right axis is for the range from 500 to $700 \mathrm{~nm}$. 
Table 3.4.1. Comparison of observed optical absorbance maxima, A407/280 ratio and heme/subunit ratio for purified KatGs.

\begin{tabular}{lcccc}
\hline KatG & $\begin{array}{c}\text { Sorêt maximum } \\
(\mathrm{nm})\end{array}$ & $\mathrm{A}_{407 / 280 \text { ratio }}$ & $\begin{array}{c}\text { Theoretical } \\
\mathrm{A}_{407 / 280} \text { ratios }^{\mathrm{a}}\end{array}$ & $\begin{array}{c}\text { Heme/subunit } \\
\text { ratio }\end{array}$ \\
\hline MtKatG & 408 & 0.60 & 0.61 & 0.98 \\
Ser315Thr & 408 & 0.54 & 0.61 & 0.88 \\
Ser315Asn & 408 & 0.56 & 0.61 & 0.92 \\
\hline
\end{tabular}

${ }^{a} \varepsilon$ at $280 \mathrm{~nm}$ calculated based on the $\varepsilon_{\mathrm{TRP}}=5500 \mathrm{M}^{-1} \mathrm{~cm}^{-1}$ and $\varepsilon_{\mathrm{TYR}}=1490 \mathrm{M}^{-1} \mathrm{~cm}^{-1}$ and $\varepsilon$ at $407 \mathrm{~nm}$ for heme estimated to be $100,000 \mathrm{M}^{-1} \mathrm{~cm}^{-1}$ 
Table 3.4.2. Comparison of catalase and peroxidase activities of MtKatGs and Ser315Thr and Ser315Asn variants.

\begin{tabular}{|c|c|c|c|}
\hline \multirow[b]{2}{*}{ KatG } & \multirow[b]{2}{*}{ Catalase (units $/ \mathrm{mg})^{\mathrm{a}}$} & \multicolumn{2}{|c|}{ Peroxidase } \\
\hline & & $\begin{array}{c}\text { ABTS }^{\mathrm{b}} \\
\text { (units/mg) }^{\mathrm{a}}\end{array}$ & $\begin{array}{c}\text {-dianisidine } \\
(\text { units } / \mathrm{mg})^{\mathrm{a}}\end{array}$ \\
\hline MtKatG & $4450 \pm 140$ & $10 \pm 2.5$ & $8.8 \pm 1.5$ \\
\hline Ser315Thr & $4200 \pm 480$ & $4.3 \pm 0.3$ & $5.4 \pm 0.8$ \\
\hline Ser315Asn & $1880 \pm 180$ & $1.3 \pm 0.2$ & $3.1 \pm 0.4$ \\
\hline
\end{tabular}

${ }^{a}$ Data expressed as $\mu$ mole $\mathrm{H}_{2} \mathrm{O}_{2} \cdot \mathrm{min}^{-1} \cdot \mathrm{mg}^{-1}$

${ }^{a}$ Data expressed as $\mu$ mole ABTS. $\mathrm{min}^{-1} \cdot \mathrm{mg}^{-1}$

${ }^{a}$ Data expressed as $\mu$ mole $o$-dianisidine. $\min ^{-1} \cdot \mathrm{mg}^{-1}$ 
$50 \%$ reduction in the $V_{\max }^{r}$ values as compared to that of the native enzyme. The $K_{\mathrm{m}}$ of Ser315Thr for ABTS is 1.5 times higher and that for Ser315Asn is $25 \%$ lower as compared to the native enzyme. The $K_{\mathrm{m}}$ values for $\mathrm{H}_{2} \mathrm{O}_{2}$ (required only for generation of the compound I in the peroxidase activity) are similar for both native enzyme and Ser315Thr variant whereas the Ser315Asn variant has a $K_{\mathrm{m}}$ two times higher than the native enzyme.

\subsubsection{Comparison of heme inhibition by cyanide and azide.}

Both variants are inhibited by cyanide and azide, classical heme protein inhibitors (Figure 3.4.7 and Table 3.4.5). Ser315Thr shows 50\% inhibition of catalase activity at 55 $\mu \mathrm{M}$ concentration of $\mathrm{KCN}$ while the Ser315Asn variant requirs $126 \mu \mathrm{M} \mathrm{KCN}$ for $50 \%$ inhibition as compared to $112 \mu \mathrm{M}$ needed for native $\mathrm{MtKatG}$. For $\mathrm{NaN}_{3}$, both variants exhibit 22-25 times higher $\mathrm{IC}_{50}$ values than the same needed for native MtKatG (Figure 3.4.7 and Table 3.4.5) consistent with the mutation constricting the substrate access channel.

\subsubsection{Effect of Ser315 mutation on NADH oxidase activity and isoniazid activation.}

Neither Ser315Thr nor Ser315Asn exhibit NADH oxidase activity at $340 \mathrm{~nm}$ although Ser315Thr shows very low levels of radical formation using NADH as substrate and NBT as radical sensor (Figure 3.4.8 and Table 3.4.6). In addition a low level of hydrazine lyase activity is associated with both Ser315Thr and Ser315Asn as compared to the native enzyme. Similar results are evident when $2 \mu \mathrm{M}$ of $\mathrm{Mn}^{2+}$ was added to the 

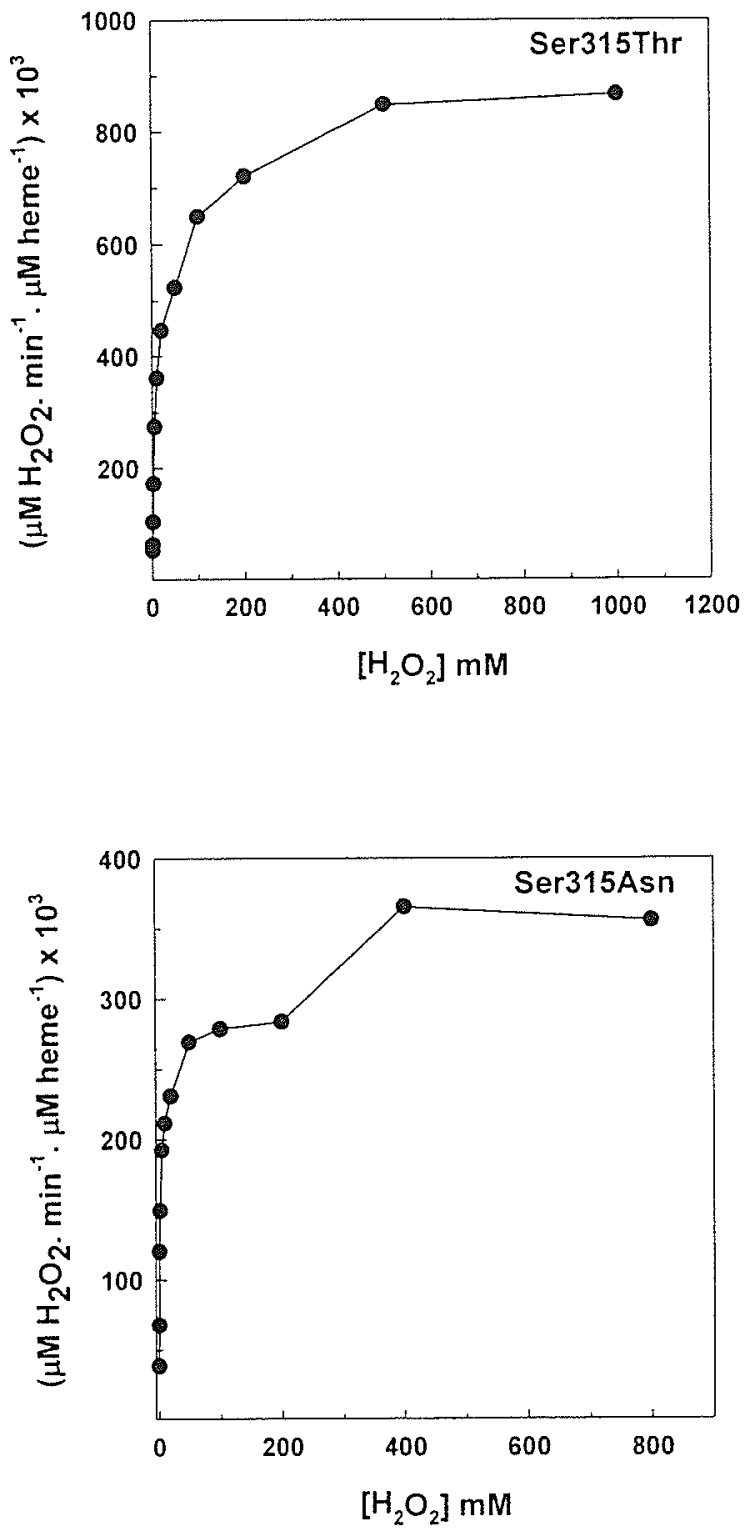

Figure 3.4.3. Effect of $\mathrm{H}_{2} \mathrm{O}_{2}$ concentration on the initial catalatic velocities of $\mathrm{S} 31 \mathrm{~T}$ and Ser315Asn variants of MtKatG. 
Table 3.4.3. Comparison of the observed catalatic kinetic parameters of purified MtKatG and Ser315Thr and Ser315Asn variants using $\mathrm{H}_{2} \mathrm{O}_{2}$ as substrate.

\begin{tabular}{ccccc}
\hline KatG & $V_{\max }{ }^{\mathrm{a}}$ & $\begin{array}{c}K_{\mathrm{m}} \\
(\mathrm{mM})\end{array}$ & $\begin{array}{c}k_{\text {cat }} \\
\left(\mathrm{s}^{-1}\right)\end{array}$ & $\begin{array}{c}k_{\text {cat }} / K_{\mathrm{m}} \\
\left(\mathrm{M}^{-1} \mathrm{~s}^{-1}\right)\end{array}$ \\
\hline MtKatG & $460 \pm 22 \times 10^{3}$ & $2.4 \pm 0.5$ & 7600 & $3.2 \times 10^{6}$ \\
Ser315Thr & $440 \pm 27 \times 10^{3}$ & $13 \pm 2.6$ & 7300 & $0.6 \times 10^{6}$ \\
Ser315Asn & $240 \pm 32 \times 10^{3}$ & $1.4 \pm 0.9$ & 4000 & $2.9 \times 10^{6}$ \\
\hline
\end{tabular}

${ }^{\mathrm{a}} \mathrm{V}_{\max }$ is expressed as $\mu$ moles of $\mathrm{H}_{2} \mathrm{O}_{2}$ decomposed $\min ^{-1} \mu$ mole heme ${ }^{-1}$ 

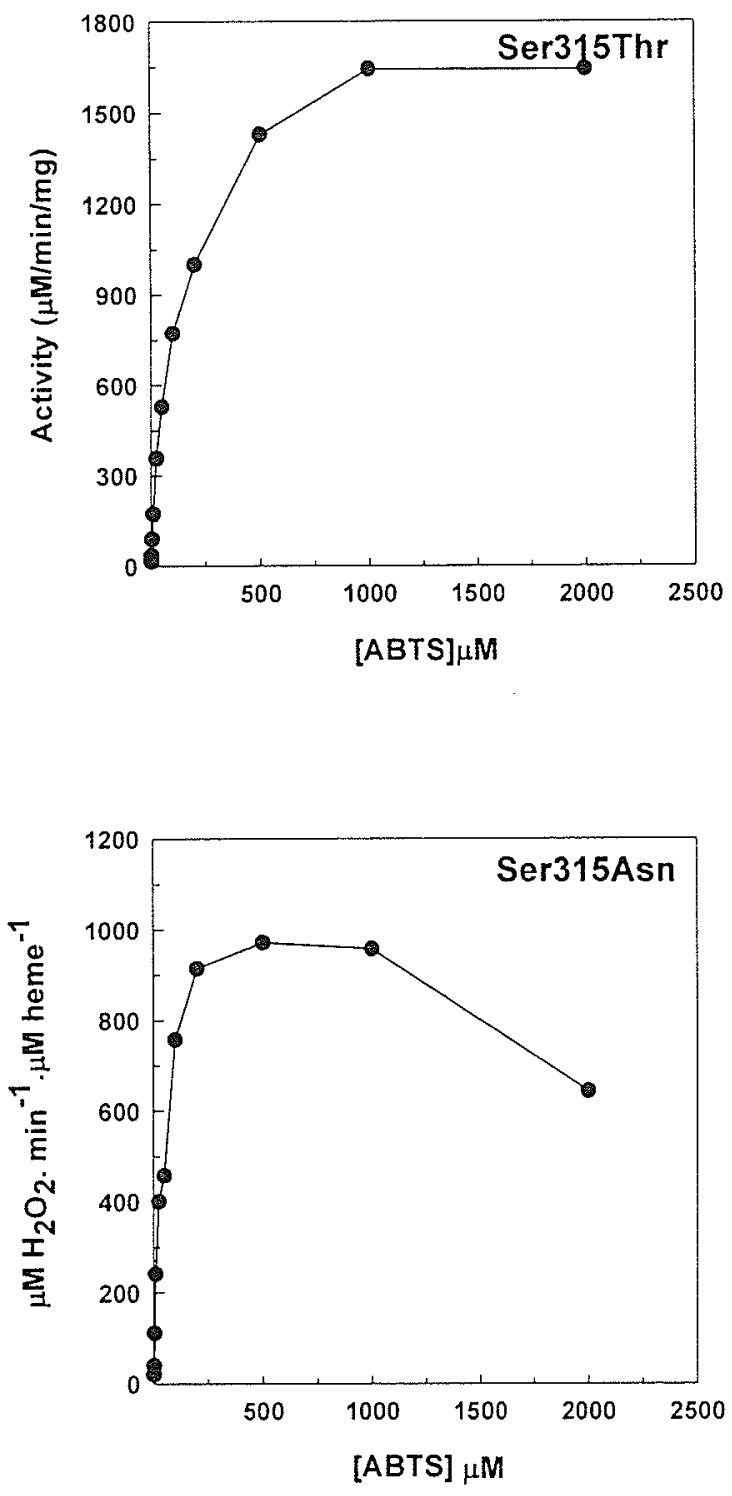

Figure 3.4.4. Effect of ABTS concentration on the initial catalatic velocities of Ser315Thr and Ser315Asn variants of MtKatG. 

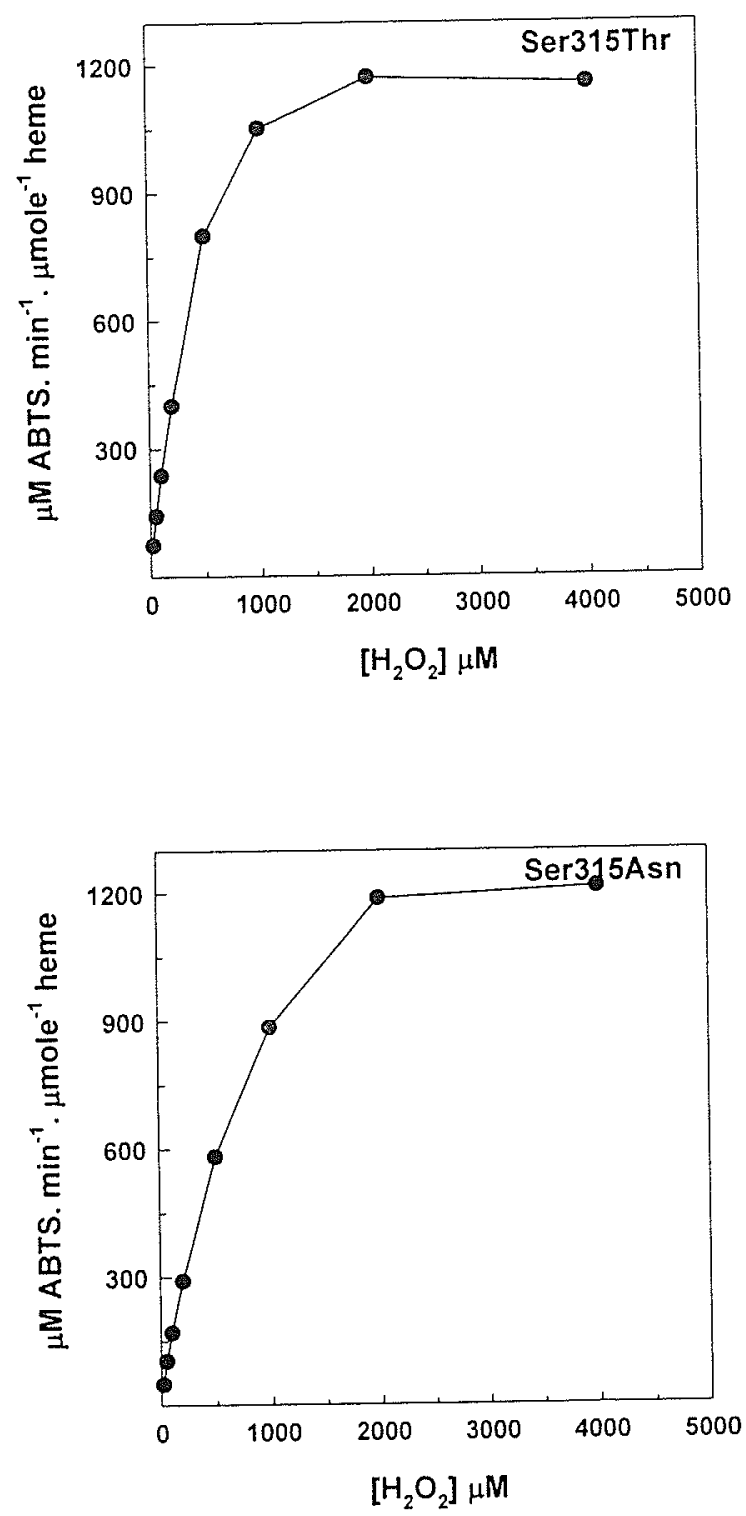

Figure 3.4.5. Effect of $\mathrm{H}_{2} \mathrm{O}_{2}$ concentration on the initial peroxidatic velocities of Ser315Thr and Ser315Asn variants of MtkatG. 
Table 3.4.4. Comparison of the observed peroxidatic kinetic parameters of purified $\mathrm{MtKatG}$ and Ser315Thr and Ser315Asn variants using ABTS as substrate.

\begin{tabular}{cccccc}
\hline KatG & $V_{\max }{ }^{a}$ & $\begin{array}{c}K_{\mathrm{m}}{ }^{\mathrm{b}} \\
(\mu \mathbf{M})\end{array}$ & $\begin{array}{c}K_{\mathrm{m}}{ }^{\mathrm{c}} \\
(\mu \mathrm{M})\end{array}$ & $\begin{array}{c}k_{\text {cat }} \\
\left(\mathbf{s}^{-\mathbf{1}}\right)\end{array}$ & $\begin{array}{c}k_{\text {cat }} / K_{\mathrm{m}} \\
\left(\mathbf{M}^{-1} \mathbf{s}^{-1}\right)\end{array}$ \\
\hline MtKatG & $1870 \pm 53$ & $67 \pm 7$ & $360 \pm 102$ & $31 \pm 0.9$ & $4.5 \times 10^{5}$ \\
Ser315Thr & $940 \pm 32$ & $100 \pm 16$ & $380 \pm 59$ & $42 \pm 0.5$ & $5.0 \times 10^{5}$ \\
Ser315Asn & $830 \pm 30$ & $50 \pm 3$ & $740 \pm 101$ & $14 \pm 0.5$ & $2.8 \times 10^{5}$ \\
\hline
\end{tabular}

${ }^{a} V_{\max }$ is expressed as $\mu$ moles of ABTS decomposed $\min ^{-1} \mu$ mole heme ${ }^{-1}$

${ }^{\mathrm{b}} K_{\mathrm{m}}$ shown for $\mathrm{ABTS},{ }^{\mathrm{c}} K_{\mathrm{m}}$ shown for $\mathrm{H}_{2} \mathrm{O}_{2}$ 

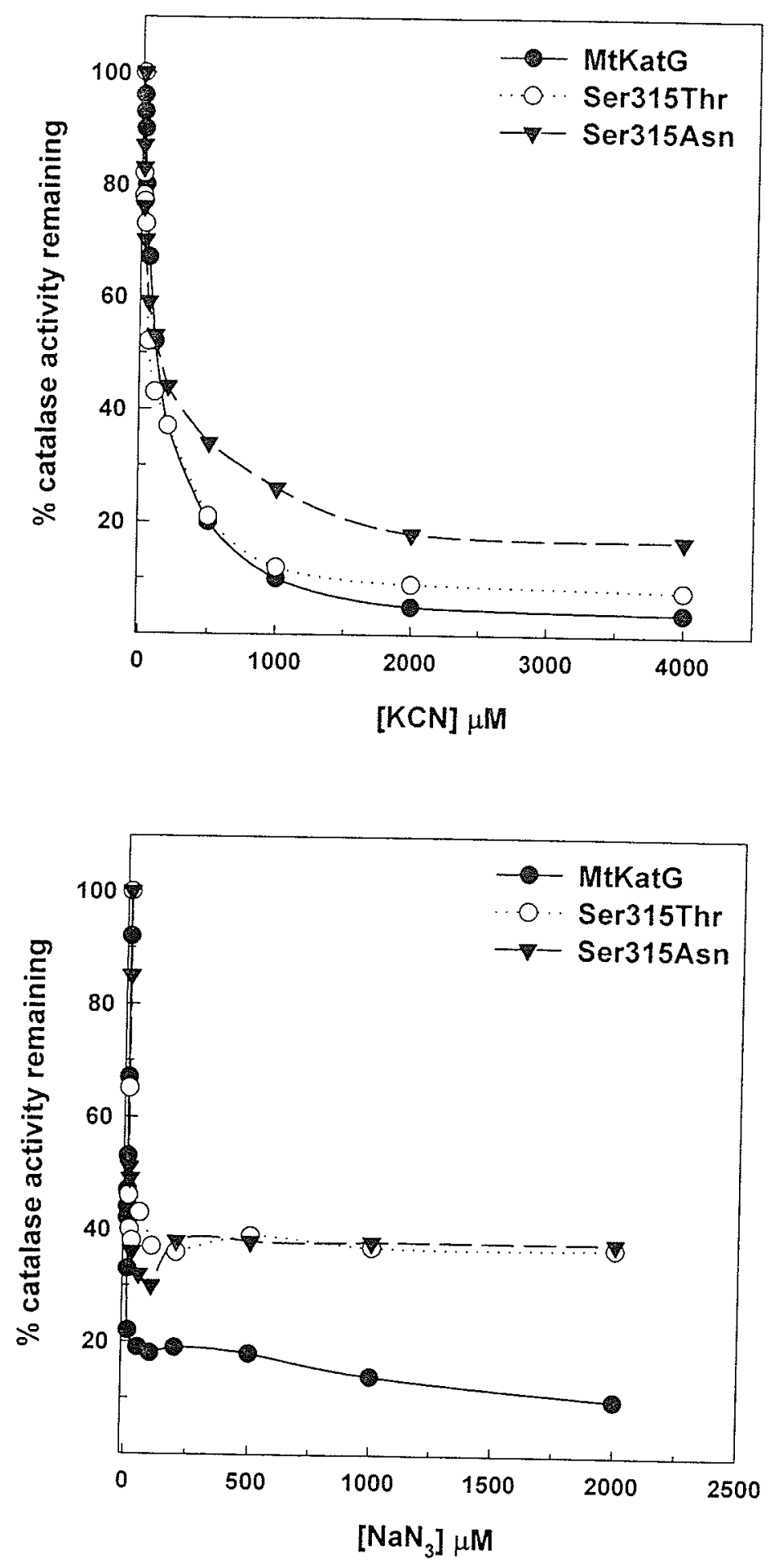

Figure 3.4.6. Comparison of sensitivity of $\mathrm{MtKatG}$ and its variants to cyanide $(\mathrm{KCN})$ or azide $\left(\mathrm{NaN}_{3}\right)$. 
Table 3.4.5. Comparison of sensitivity of $\mathrm{KatG}$ and its variants to cyanide $(\mathrm{KCN})$ or azide $\left(\mathrm{NaN}_{3}\right)$.

\begin{tabular}{ccc}
\hline KatG & $\begin{array}{c}{[\mathrm{KCN}] \text { causing } 50 \%} \\
\text { inhibition }(\mu \mathrm{M})\end{array}$ & $\begin{array}{c}{\left[\mathrm{NaN}_{3}\right] \text { causing } 50 \%} \\
\text { inhibition }(\mu \mathrm{M})\end{array}$ \\
\hline MtKatG & 98 & 0.2 \\
Ser315Thr & 55 & 5 \\
Ser315Asn & 110 & 4.5 \\
\hline
\end{tabular}


Figure 3.4.7. NADH oxidation by MtKatG and variants Ser315Thr and Ser315Asn. $a$, the rates of radical production in a solution containing $100 \mu \mathrm{M} \mathrm{NADH}, 1.2 \mu \mathrm{M}$ enzyme, $200 \mu \mathrm{M}$ NBT and $50 \mathrm{mM}$ Tris, $\mathrm{pH} 8.75$, are followed by formazan appearance measured at $560 \mathrm{~nm}$. Separate assays contain no enzyme (control), MtKatG, S315Tand Ser315Asn. $b$, the rates of NADH oxidation in a solution containing $100 \mu \mathrm{M} \mathrm{NADH}, 1.2 \mu \mathrm{M}$ enzyme, and $50 \mathrm{mM}$ Tris, $\mathrm{pH} 8.75$ were followed by NADH disappearance measured at $340 \mathrm{~nm}$. Separate assays contain no enzyme (control), MtKatG, S315Tand Ser315Asn. 


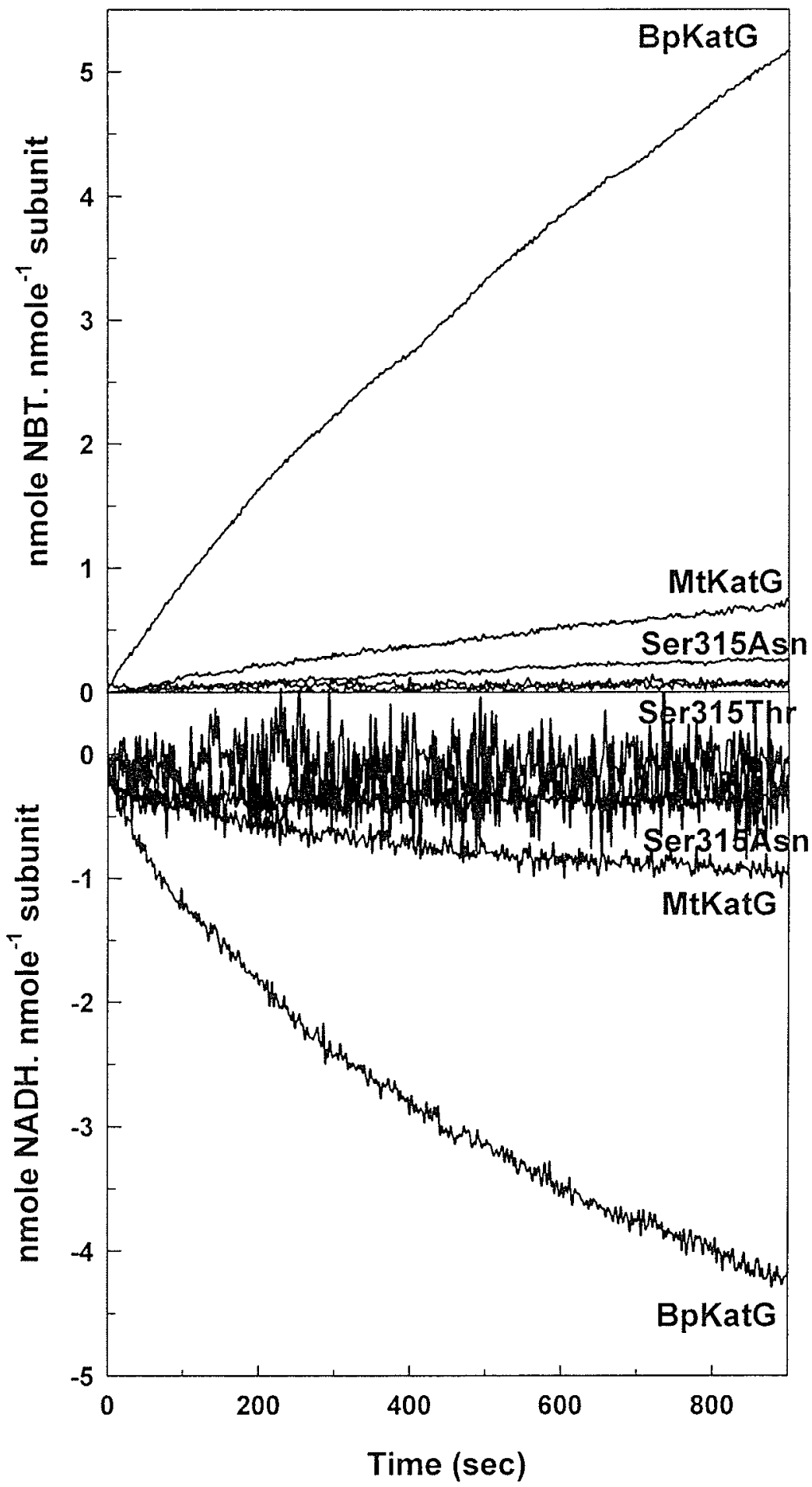


Figure 3.4.8. Removal of hydrazine form isoniazid by $\mathrm{MtKatG}$ and variants. $a$, radical generation in a solution containing $10 \mathrm{mM} \mathrm{NNH}, 200 \mathrm{mM} \mathrm{NBT}$ as radical sensor in 50 $\mathrm{mM}$ Tris, pH 8.0, was followed by formazan appearance measured at $560 \mathrm{~nm}$. Separate assays contain no enzyme (control), MtKatG and variants Ser315Thr and Ser315Asn. $b$, Similar assay conditions were employed to determine the effect of $\mathrm{Mn}^{2+}$ on the hydrazine lyase reaction. 

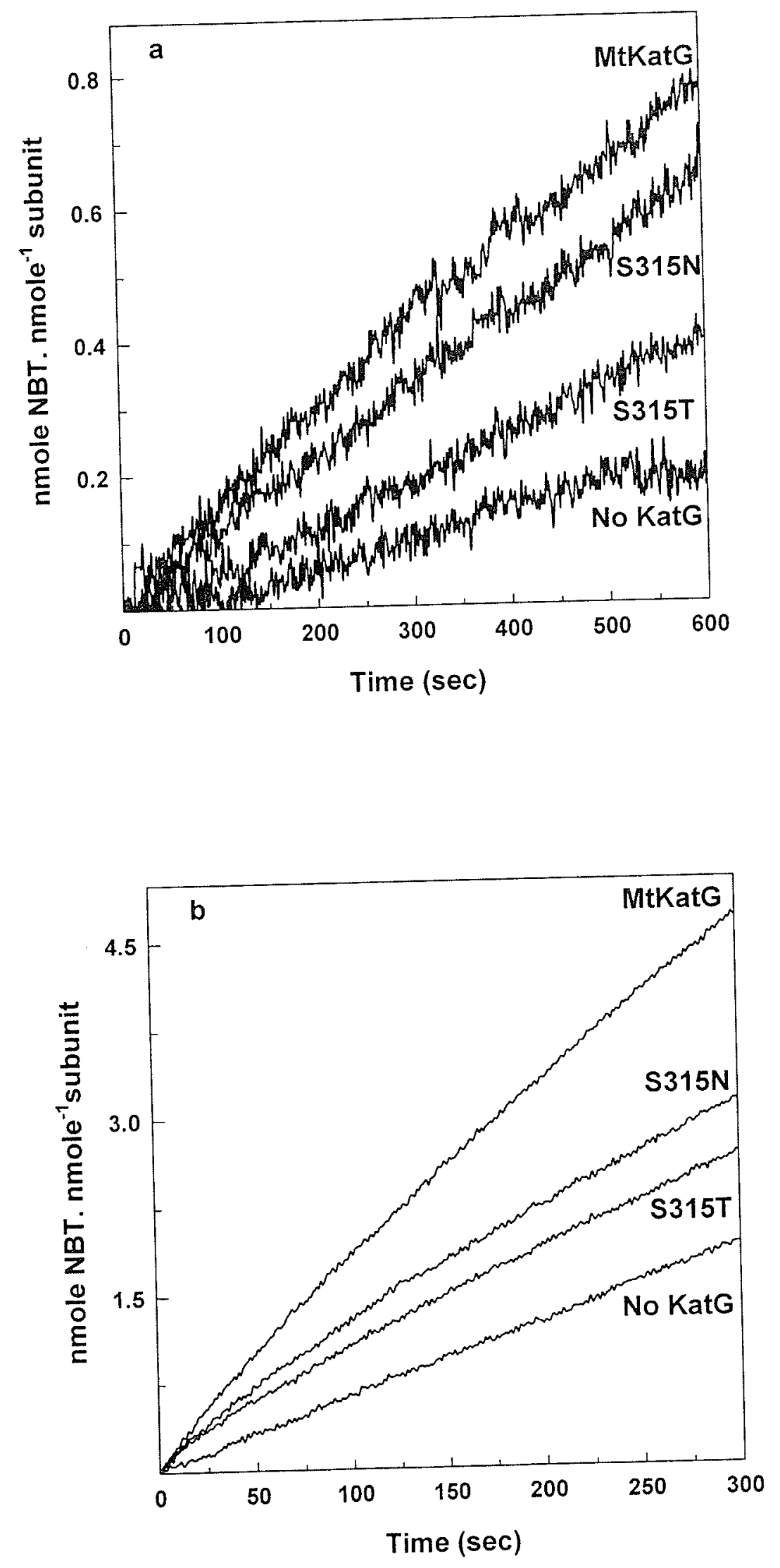
Figure 3.4.9. Effect of NADH on the hydrazine lyase activity by MtKatG and variants $a$, Isonicotinoyl-NAD synthase activity by MtKatG and variants $b$. 

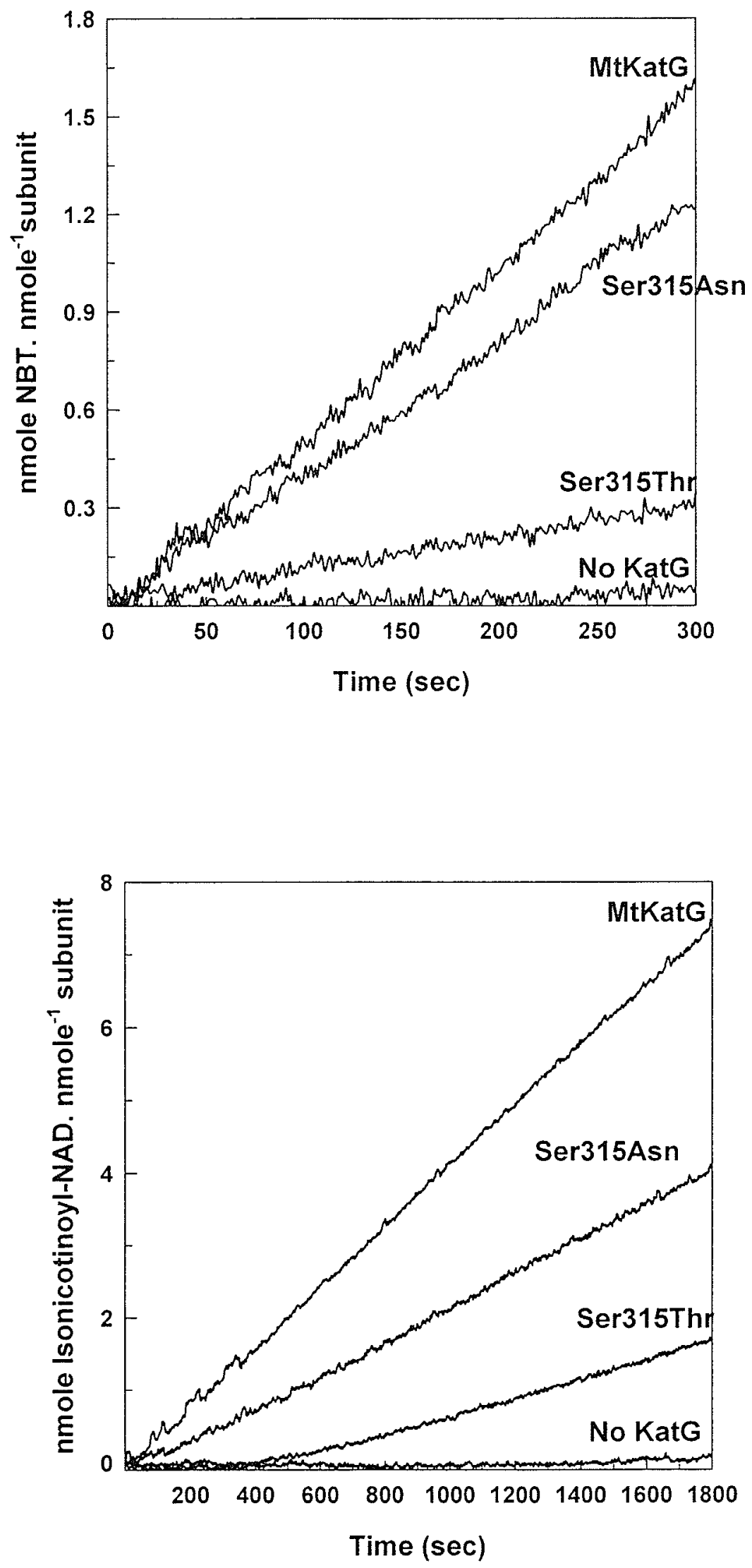
Table 3.4.6. Comparison of NADH oxidase activity, hydrazine lyase, and synthase activity by $\mathrm{MtKatG}$ and variants.

\begin{tabular}{lccc}
\hline & MtkatG $^{\mathrm{a}}$ & Ser315Thr $^{\mathrm{a}}$ & Ser315Asn $^{\mathrm{a}}$ \\
\hline NADH oxidase $\left(\mathbf{A}_{\mathbf{5 6 0}}\right)$ & $100 \pm 15$ & ND & 20 \\
NADH oxidase (A $\mathbf{3 4 0})$ & $30 \pm 3$ & ND & ND \\
INH hydrazine lyase & $78 \pm 9$ & $27 \pm 3$ & $60 \pm 5$ \\
INH + NADH & $1140 \pm 76$ & $57 \pm 5$ & $115 \pm 6$ \\
INH + Mn & $460 \pm 5$ & $250 \pm 15$ & $320 \pm 10$ \\
Isonicotinoyl-NAD synthase & $93 \pm 6$ & $20 \pm 4$ & $30 \pm 8$ \\
\hline
\end{tabular}

${ }^{a}$ Data expressed as pmole. $\min ^{-1}$. nmole ${ }^{-1}$ subunit

${ }^{b}$ Data expressed as pmole. min-1. mmole ${ }^{-1}$ subunit

$\mathrm{ND}$, not determined 
reaction mixture (Figure 3.4.9 and Table 3.4.6) demonstrating the significance of the native enzyme structure in drug activation. Following this trend, the rate of radical production from a reaction mixture including INH and NADH is 20 times lower for Ser315Thr and 10 times lower for Ser315Asn variant as compared to the native enzyme (Figure 3.4.10 and Table 3.4.6). Most significantly, the isonicotinoyl-NAD synthase activity is almost five times lower for Ser315Thr variant and three times lower for Ser315Asn variant compared to the native enzyme. These results are consistent with the reports demonstrating the lower sensitivity to isoniazid of clinical isolates carrying Ser315 mutation on kat $G$ of $M$. tuberculosis. 


\section{Discussion}

\subsection{Location of conserved residues in catalase-peroxidases}

Because the in vivo peroxidase substrates remain unidentified, possible substratebinding sites, for as yet unidentified substrates, was a topic for speculation in the original report of the BpKatG structure (Carpena et al., 2002). Unambiguous identification of such sites will have to await a crystal structure determination of protein-ligand complexes. Evidence for a diversity of substrate binding sites in KatGs is shown in the high frequency of highly conserved residues (Table 4.1) and their broad distribution throughout the KatG subunit including on the surface (Figure 4.1). Over 27 and $18 \%$ of the residues, respectively, in the catalytic $\mathrm{N}$-terminal domain and noncatalytic C-terminal domain of the KatG subunit are identical in more than $95 \%$ of the sequences available for KatGs. This is compared with the $8-14 \%$ frequency of nearly identical residues, all located near the active sites in pyruvate kinases, catalases, peroxidases, CuZn SODs, and FeMn SODs. Seven KatGs used in this study also have $50-70 \%$ similarity in the amino acid sequence (Figure 4.2) and, the four crystal structures that have appeared so far (Yamada et al., 2002; Carpena et al., 2003; Vada et al., 2002; and Bertrand et al., 2004) exhibit a high degree of structural integrity particularly in the vicinity of heme (Figure 1.3). All four KatGs adopt similar folds with MtKatG sharing 55\% identity and 69\% similarity with $\mathrm{HmKatG}$ and $66 \%$ identity and $77 \%$ similarity with BpKatG (Bertrand et al., 2004). C $\alpha$ backbone superpositions show root mean square deviations of 1.18 and $0.82 \AA$ between $\mathrm{MtKatG}$ with $\mathrm{HmKatG}$ or $\mathrm{BpKatG}$, respectively. Active site residues are conserved and align well in all three structures, and there are few structural differences in the three-dimensional arrangement of the polypeptide chains. In 


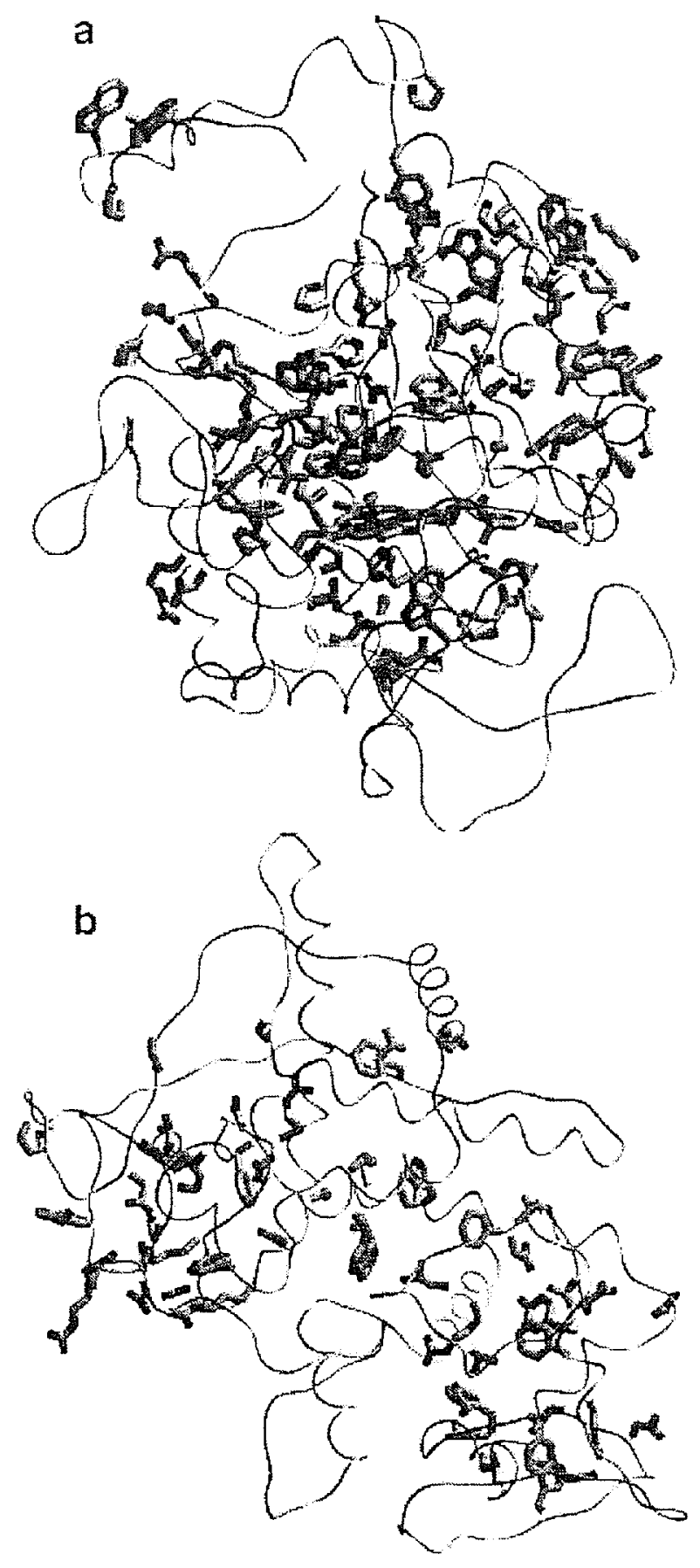

Figure 4.1. Location of residues in BpKatG that are identical in more than $95 \%$ of the 53 catalase-peroxidase sequences available. The distribution in the $\mathrm{N}$-terminal domain is shown in $a$, and the distribution in the C-terminal domain is shown in $b$. The domains are treated separately for a clearer representation of the differences. 
Table 4.1 Comparison of the percentage of highly conserved residues ( $>95 \%$ identical) in KatGs compared to other families of proteins

\begin{tabular}{|c|c|c|c|c|c|c|c|}
\hline & \multicolumn{2}{|c|}{ KatG } & \multicolumn{3}{|c|}{ SOD } & \multirow{2}{*}{ Peroxidase } & \multirow{2}{*}{$\begin{array}{c}\text { Pyruvate } \\
\text { kinase }\end{array}$} \\
\hline & $\mathrm{N}$-terminal & C-terminal & & $\mathrm{CuZn}$ & $\mathrm{FeMn}$ & & \\
\hline No. in family & $52^{\mathrm{a}}$ & $52^{\mathrm{a}}$ & $228^{\mathrm{a}}$ & $22^{b}$ & $25^{\mathrm{c}}$ & $24^{d}$ & $28^{\mathrm{e}}$ \\
\hline Length & 390 & 322 & 490 & 155 & 238 & 294 & 470 \\
\hline $\begin{array}{l}\text { No. with }>95 \% \\
\text { identity }\end{array}$ & 107 & 59 & 57 & 22 & 22 & 24 & 59 \\
\hline $\begin{array}{l}\text { Percentage with } \\
>95 \% \text { identity }\end{array}$ & 27.4 & 18.3 & 11.6 & 14.2 & 9.2 & 8.2 & 12.3 \\
\hline
\end{tabular}

${ }^{a}$ Accession numbers are described by Klotz and Loewen (Klotz and Loewen, 2003)

${ }^{b}$ The accession numbers for the sequences used are: AA054860, AAR15417 AB087845, AF312586, AF312588, AF318938, AJ581746, AY428604, AY434497, BAC96317 BC061861, CAC28938 Jo2658, J04087, L13778, M15175, M84013, NM011435, NM174615, NP012638, NP231223, NP940620, P24704, X97766, and XM358882

${ }^{c}$ The accession numbers for the sequences used are: AAN16456 AA057908, AB093035, AB109302, AF318020, AY314980, J03511, L11707, L25675, M33119, M60401, M74242, M94879, M96560, NM013671, NM057577, NM059889, NP011872, NP231679, NP232322, NP704405, NP940564, Q8K6Y8, and X03951

${ }^{d}$ The accession numbers for the sequences used are: AB027752, AB009084, AB022273, AB024437, AB027752, AB027753, AF039027, AF109123, AF139910, AF149278, AF155124, AF159380, AF159629, AF175710, AJ003141, AJ011939, D11102, D11337, D14442, D83669, D84400, D90115, M60729, Y16773, and Y17192

${ }^{e}$ The accession numbers for the sequences used are: AAB47952 AAH16619 AAH19265 AAHY25737, AAH61541 AAO57788 AAQ57928 BAC91436 BAD01636 CAA24631 CAE07913 CAE14987 CAE20854 NP230139, NP231642, NP416191, NP416368, NP703926, NP721573, NP881869, NP934082, NP939895, Q8Z6K2, Q9Z9B4, Q89AI8, XP224416, XP341924, ZP00026409, ZP00078743, and ZP000122573 
particular, the covalent modification linking the distal Trp 111, Tyr238 and Met264

residues (BpKatG numbering), which are fully conserved in all seven KatGs used in this study, plays a crucial role in the modulation of catalase activity in these heme proteins (Donald et al., 2003; Jakospitch et al., 2003d). This modification is unique to KatGs and has been reported in all four crystal structures and confirmed using mass spectrometry (Donald et al., 2003; Jakospitch et al., 2003; Ghiladi et al., 2005; and Ghiladi et al., 2005a). Despite these apparent structural similarities, this study demonstrates significant differences upon interaction of KatGs with various substrates and in the catalytic efficiency of the reactions indicating the importance of subtle changes within the KatG backbone in the regulation of activities.

\subsection{Overview and comparison of catalase and peroxidase activities}

Seven KatGs studied demonstrated different levels of catalase and peroxidase activities ranging from $3700-6000 \mathrm{U} / \mathrm{mg}$. The enzyme kinetics reveal differences in $V_{\max }$ and $K_{\mathrm{m}}$ values. For example, the catalatic $K_{\mathrm{m}}$ for $\mathrm{H}_{2} \mathrm{O}_{2}$ of $\mathrm{MtKatG}$ is $2.4 \mathrm{mM}$, but for BpKatG and EckatG, the $K_{\mathrm{m}} \mathrm{s}$ are 4.3 and $4.2 \mathrm{mM}$ (Table 3.6). Similarly the turnover rates for all seven KatGs vary from 7600 to $17300 \mathrm{~s}^{-1}$ and $k_{\text {cat }} / K_{\mathrm{m}}$ values also vary from 2 to $5.6 \mathrm{M}^{-1} \mathrm{~s}^{-1}$ (Table 3.6). These variations are significant and a plausible explanation could be the differences in the substrate access channel. In KatGs there are conserved acidic residues in the main access channel: Glu242 (BpKatG numbering) at the entrance and Asp141 at the 3-4 $\AA$ wide constriction (Smuelvich et al., 2006). Both residues are critical for stabilizing the solute matrix in the channel and for orienting the water dipoles. In addition, Asp141 controls the access to the distal residues Arg108, His112 and Trp111. 
These residues are conserved in the seven KatGs used in this study. Thus, the differences in the affinity for the substrate could also be arising from the presence of some other access route for the substrate apart from the main channel. Interestingly there are not significant differences in the $\mathrm{pH}$ optimum for the catalase activity which remains between pH 6.25 and 6.5 for seven KatG studies. This suggests that highly conserved active site residues modulate the catalase activity in a similar fashion and require a similar $\mathrm{pH}$ environment irrespective of the type of $\mathrm{KatG}$.

Although the in vivo peroxidatic substrate is still unknown, various organic electron donors such as ABTS and o-dianisidine are used as in vitro peroxidatic substrates. Each KatG has a different affinity for the same substrate such as ABTS, where the $K_{\mathrm{m}}$ ranges from 7 to $300 \mu \mathrm{M}$ in the seven enzymes studied (Table 3.7). Binding of the same substrate with different affinity to different KatGs could be attributed to the differences in the binding sites on the protein. Even between two different substrates used in this study (ABTS and $o$-dianisidine), different kinetic constants for peroxidase activity were noted among the enzymes but exhibited similar optimum pHs (Table 3.4) suggesting a common reaction involving similar catalytic residues. The specificity constants $\left(k_{\text {cat }} / K_{\mathrm{m}}\right)$ and the $K_{\mathrm{m}}$ for $\mathrm{H}_{2} \mathrm{O}_{2}$ for compound I formation for the peroxidase activity of the seven KatGs varied $20-80$ folds from 0.5 to $40 \times 10^{5} \mathrm{M}^{-1} \mathrm{~s}^{-1}$ and $58 \mu \mathrm{M}$ to 1 $\mathrm{mM}$ respectively. The structures of the peroxidatic substrates will play an important role in their binding to the enzyme and differences in the surface topology caused by sequence variations will affect their affinity toward enzyme. Cytochrome $c$, the peroxidatic substrate for Cytochrome $c$ peroxidase $(\mathrm{CcP})$, bounds at the surface of $\mathrm{CcP}$ and the electron transfer between the enzyme and substrate occurs through protein based radicals 
formed on the CcP (Millett et al., 1995). A similar process could be a possibility with KatGs since they are closer to plant peroxidases in sequence and structure. In such a case, protein based radicals could play an important role in electron transfer from the heme to the distantly bound peroxidatic substrate. There are reports mentioning the formation of such protein based radicals in KatGs (Ivancich et al., 2003; Zhao et al., 2004) and determining the location and understanding the role of such protein radicals will be crucial in determining the peroxidatic reaction of KatGs. It is expected that studies such as this will eventually lead to the identification of the actual in vivo substrates for KatGs and as a result the significance of this activity for the organisms.

Heme inhibition by classical heme inhibitors also demonstrates different $\mathrm{IC}_{50}$ value for different KatGs. For example, SyKatG and AfKatG needed almost three times higher concentration of $\mathrm{KCN}$ as compared to that needed by BpKatG, MtKatG and EcKatG for $50 \%$ inhibition of catalase activity. On the other hand, MtKatG needed a mere $0.2 \mu \mathrm{M} \mathrm{NaN}_{3}$ for $50 \%$ inhibition in contrast to $20 \mu \mathrm{M}$ needed by AfKatG (Table 3.9).

\subsection{The oxidase, lyase and synthase activities of KatGs}

The existence of catalase, peroxidase, and oxidase activities in a single protein makes Kat $\mathrm{G}$ a complex and fascinating enzyme, an assessment further enhanced by $\mathbb{N H}$ hydrazine lyase and isonicotinoyl-NAD synthase activities. A summary of these various activities (Figure 4.2) illustrates the independence of the closely related catalase and peroxidase activities from the oxidase, hydrazine lyase, and synthase activities. Not only are the $\mathrm{pH}$ optima very different, but, unlike the catalase and peroxidase reactions, the 
oxidase, hydrazine lyase, and synthase reactions do not require $\mathrm{H}_{2} \mathrm{O}_{2}$ or involve the formation of identifiable oxidized heme intermediates. The hydrazine lyase and synthase reactions are closely linked because hydrazine lyase must occur before the ligation of $\mathrm{NAD}^{+}$to the isonicotinoyl radical, but the oxidase reaction is not mechanistically linked to any other reaction except that it very likely shares with the synthase the same $\mathrm{NAD}^{+} / \mathrm{NADH}$-binding site. In addition, the oxidase reaction may serve as a source of $\mathrm{NAD}^{+}$for the synthase reaction and $\mathrm{H}_{2} \mathrm{O}_{2}$ for the catalase and peroxidase reactions (Figure 4.3). The catalase and peroxidase functions of $\mathrm{KatG}$ are rationalized as protection against $\mathrm{H}_{2} \mathrm{O}_{2}$, and the NADH oxidase activity may present a complementary protection against molecular oxygen or a means of maintaining low cytoplasmic levels of oxygen. However, the turnover rate of the oxidase is very slow compared with the catalase and peroxidase reactions, and the oxidase activity may simply be a residual vestige of what was once a more substantial activity with metabolic significance in a particular environmental niche. Certainly, the high affinity of BpKatG for NADH is consistent with there being, or having been, a physiological significance to the activity. Among the seven KatGs in this study, the variation in NADH oxidase activity, highest in BpKatG and lower in the rest of the KatGs, might be interpreted as the result of an environmentally determined differential loss of activity. Although the KatGs can utilize NADH as a peroxidatic substrate, the NADH oxidation characterized here is clearly not a peroxidatic reaction, and it is also different in several key respects from the peroxidase-oxidase activity associated with HRP. $\mathrm{H}_{2} \mathrm{O}_{2}$ does not have a catalytic role, SOD and catalase do not inhibit the reaction, and lag periods in reaction initiation are not evident in the presence of high enzyme or low NADH concentrations. In addition, the $\mathrm{pH}$ dependence 
Figure 4.2. Amino acid sequence alignment of BpKatG, MtKatG, EcKatG, SykatG, BskatG, AfkatG, RcKatG. Sequence alignment was created using clustal. fully conserved residues; represents partially conserved residues. 

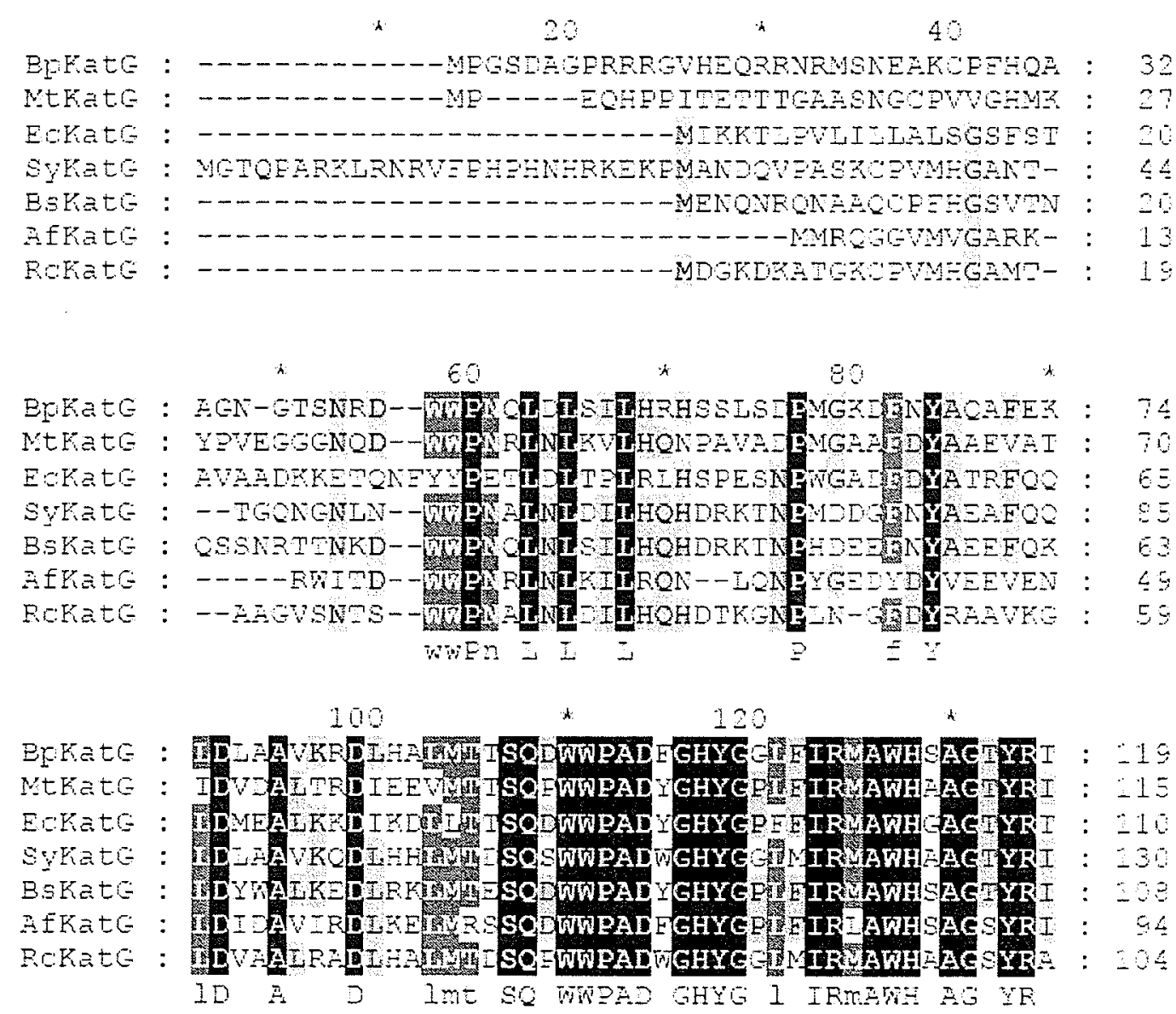

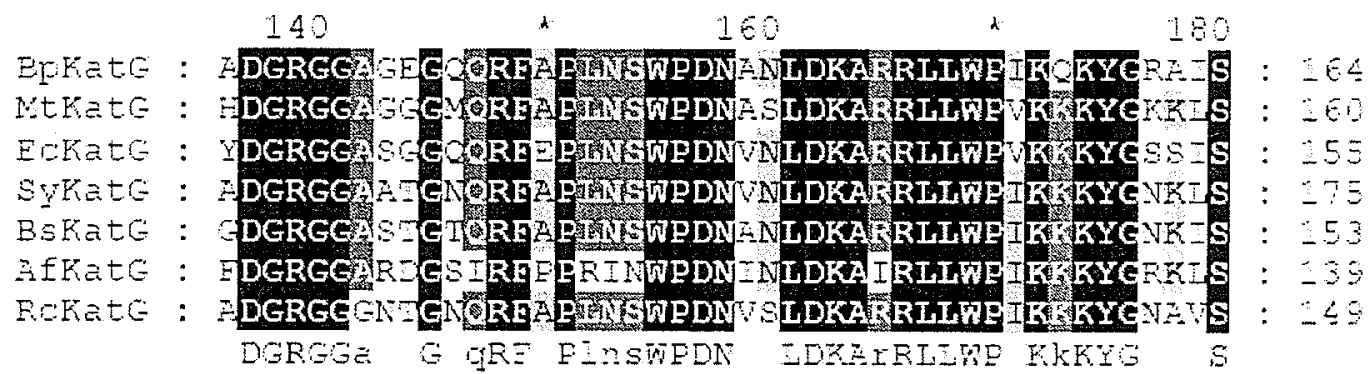




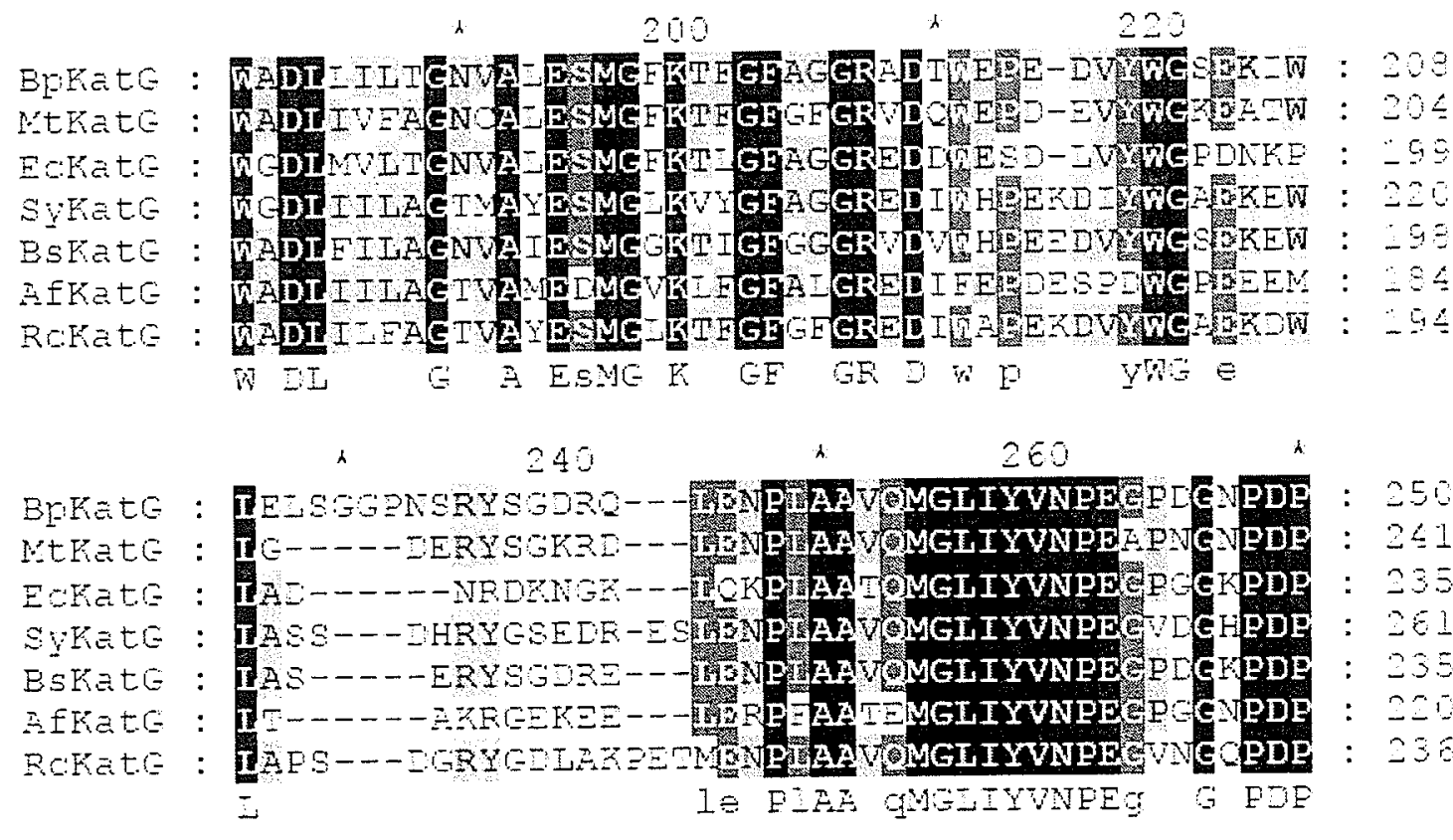

280

$\times \quad 300$

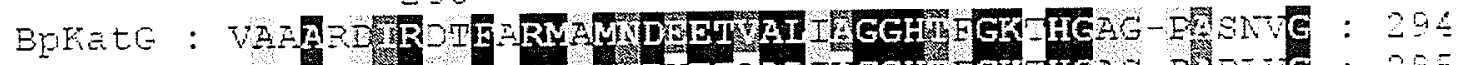

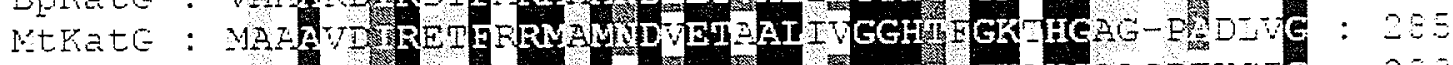

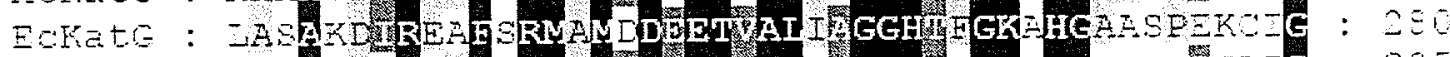

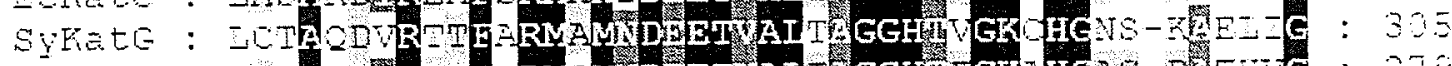

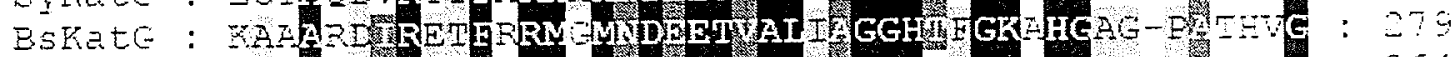

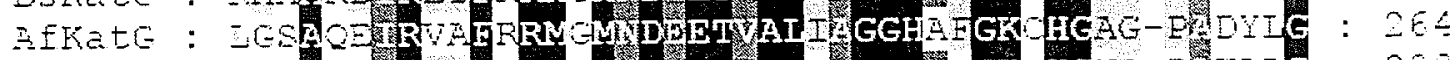
RCFatC : RRT $340 \quad x \quad 360$

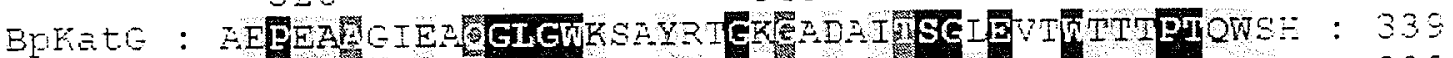

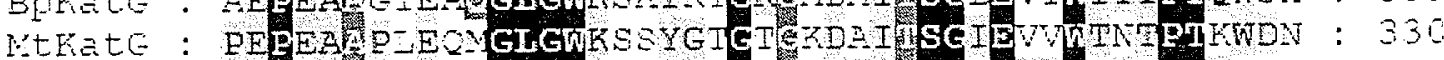

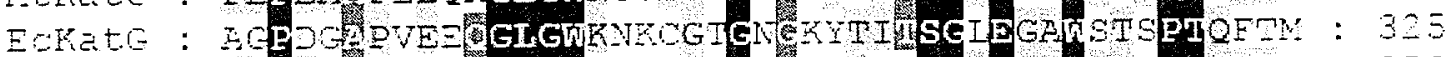

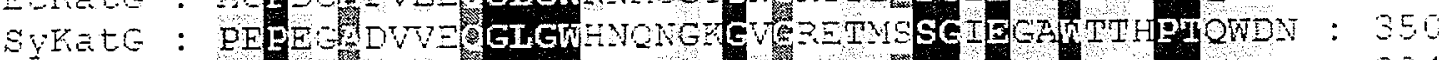

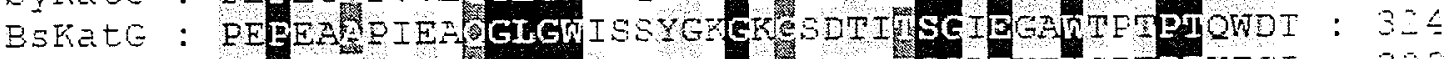

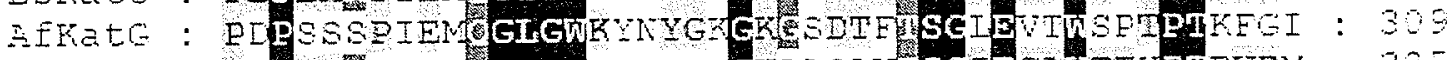

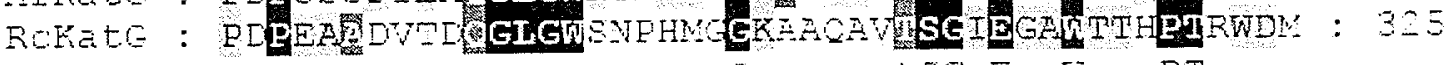

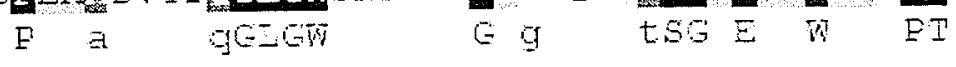



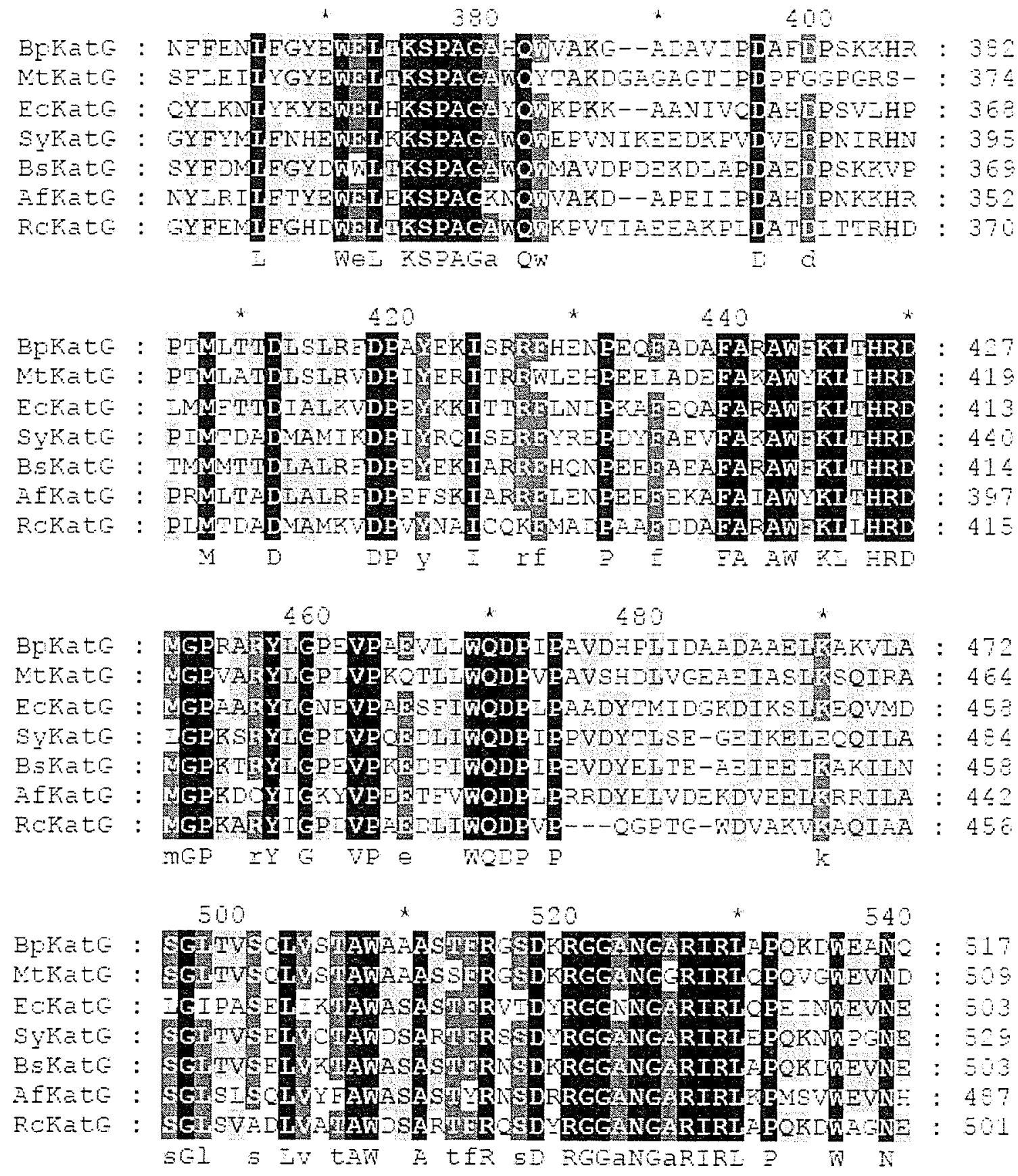

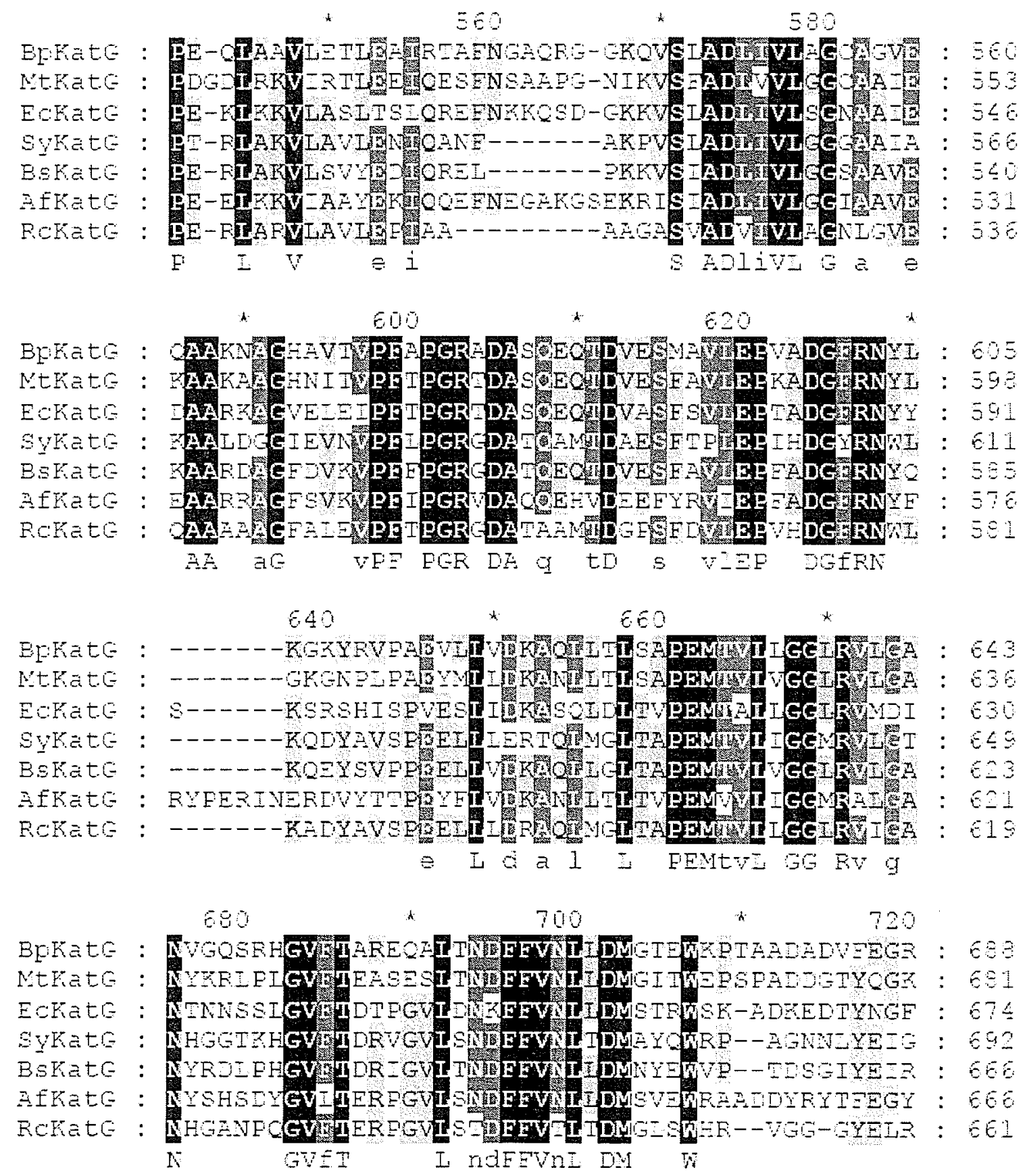


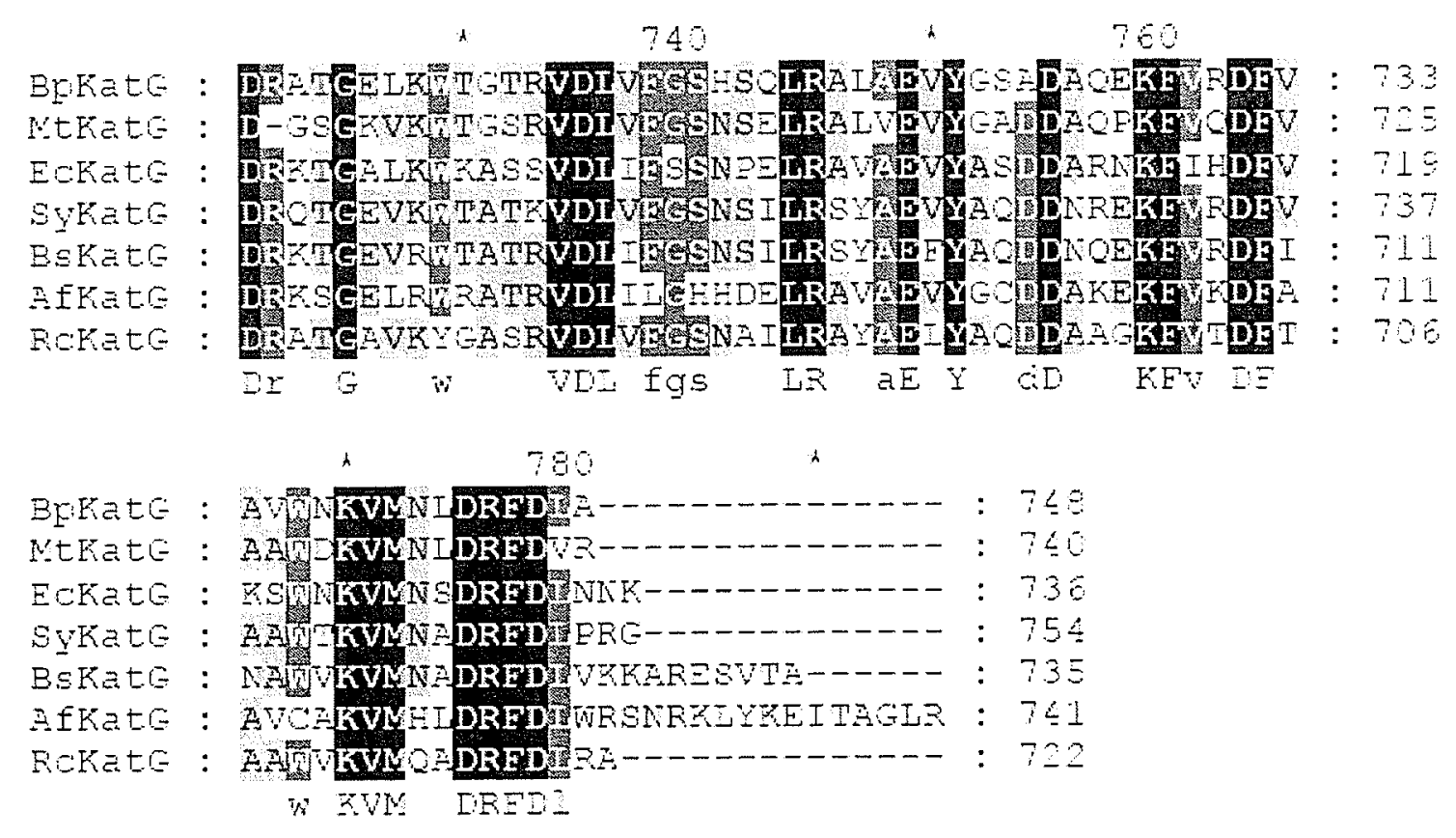


Figure 4.3. Scheme showing the relationship of the five activities of KatG. The locations of the $\mathbb{N H}$ hydrazine lyase, NADH oxidase, isonicotinoyl-NAD synthase, catalase, and peroxidase reactions are underlined. The two $\mathrm{pH}$-dependent options for the NADH oxidase reaction are also indicated. The isonicotinoyl $(a)$ and hydrazide $(b)$ radicals from the hydrazine lyase reaction are available to radical scavengers in the absence of $\mathrm{NAD}^{+}$. The di-imide and proton products from the synthase reaction, indicated in parentheses with an asterisk, have not been confirmed as products but provide a convenient and logical way to balance the reaction. The catalase and peroxidase cycles are shown at the bottom and appear to be independent of the reactions at the top except for the possible metabolism of $\mathrm{H}_{2} \mathrm{O}_{2}$ generated in the oxidase reaction (dotted line) and the possibility that molecular oxygen may also bind to the heme. 


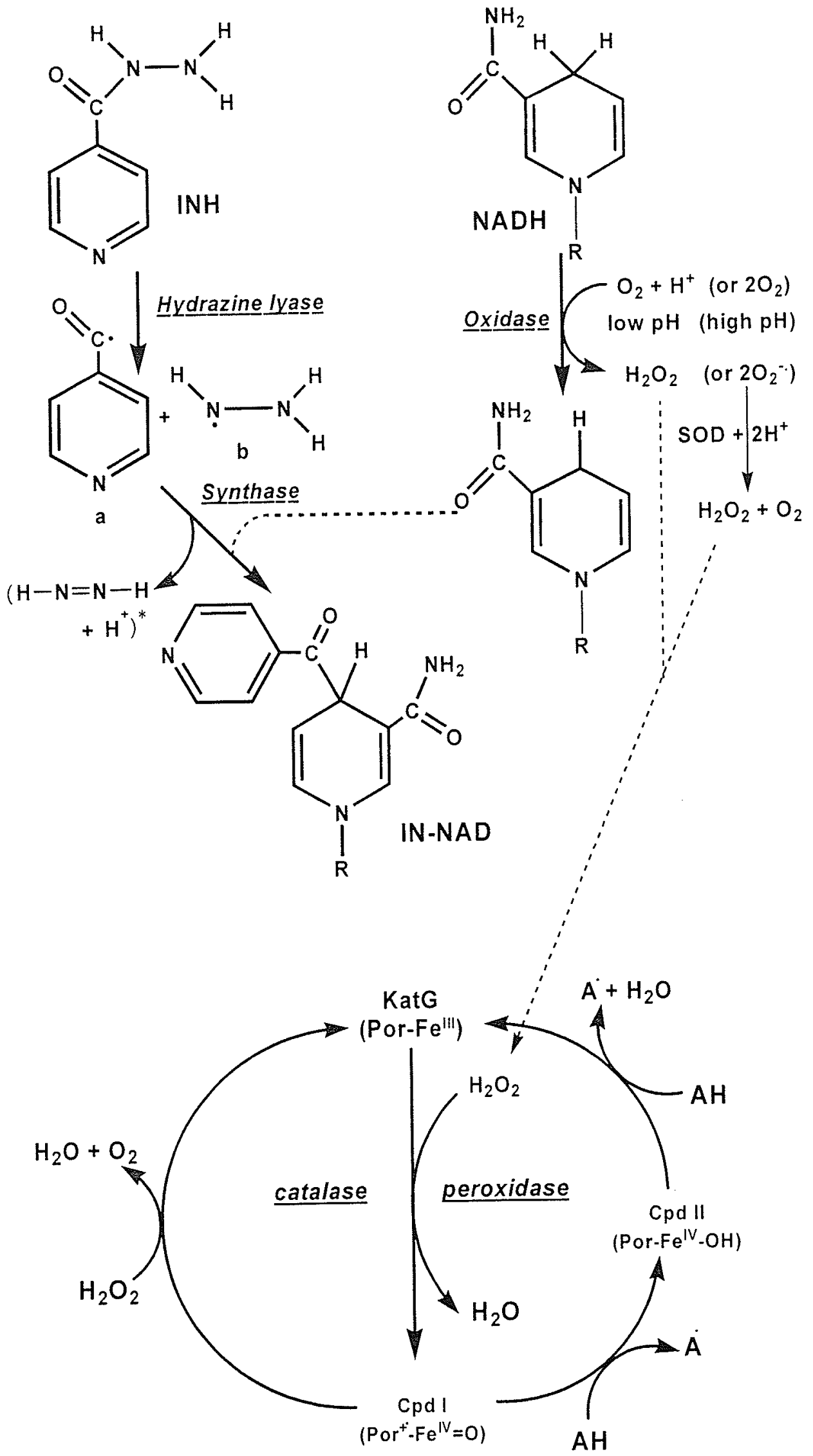


of superoxide radical formation in the oxidase reaction suggests the possibility of two reaction outcomes for molecular oxygen depending on proton availability. Below $\mathrm{pH} 8$, with protons more readily available, a two electron transfer to oxygen takes place generating $\mathrm{H}_{2} \mathrm{O}_{2}$ (Reaction 1), whereas at higher $\mathrm{pH}$, two one-electron transfers to two oxygen molecules generate two superoxide ions (Reaction 2). The protonation state of the imidazole ring of the distal side His 112 (Figure 1.5) may determine the reaction pathway through presentation of a proton to the bound oxygen in the active site. Any $\mathrm{H}_{2} \mathrm{O}_{2}$ produced via reaction 1 would rapidly oxidize the heme to compound I and subsequent reduction in catalase or peroxidase reactions would give rise to $\mathrm{H}_{2} \mathrm{O}$.

$$
\begin{aligned}
\mathrm{NADH}+\mathrm{O}_{2}+\mathrm{H}^{+} & \rightarrow \mathrm{NAD}^{+}+\mathrm{H}_{2} \mathrm{O}_{2} \\
\mathrm{NADH}+2 \mathrm{O}_{2} & \rightarrow \mathrm{NAD}^{+}+2 \mathrm{O}_{2}^{-0}
\end{aligned}
$$

The enhancement of $\mathrm{NH}$ hydrazine lyase by $\mathrm{KatG}$ and its importance in INH prodrug activation is well documented. A single mutation in Ser315 of MtKatG is sufficient to reduce the affinity of the enzyme for INH (Yu et al., 2003) and to prevent isonicotinoyl-NAD formation (Wei et al., 2003) leading to in vivo isoniazid resistance. The facts that $\mathrm{NAD}^{+}$is a competitive inhibitor of $\mathrm{NADH}$ oxidation but does not inhibit the hydrazine lyase reaction and that an $\mathrm{NH}-\mathrm{NADH}$ mixture supports a rate of radical production greater than the cumulative rates of the individual reactions suggest that $\mathrm{NADH} / \mathrm{NAD}^{+}$and INH have separate binding sites and that there is an element of synergy between the two reactions. Manganese ions (both $\mathrm{Mn}^{2+}$ and $\mathrm{Mn}^{3+}$ ) enhance both nonenzymatic and enzymatic hydrazine lyase to the extent that the manganese-mediated isonicotinoyl radical formation is faster than the KatG-mediated reaction. However, 
despite this high rate of manganese-mediated hydrazine lyase, manganese-mediated isonicotinoyl-NAD formation is negligible compared with its rapid formation in the presence of KatG. The data in this thesis do not dispute the observation that there can be a nonenzymatic origin of isonicotinoyl-NAD (Wilming and Johnsson, 1999), but under the conditions employed in this work, KatGs are a much more effective catalyst of isonicotinoyl-NAD synthesis than manganese ions.

The regulatory systems controlling KatG expression, involving OxyR in E. coli, are generally oxidative response systems, supporting the conjecture that the primary role of $\mathrm{KatG}$ is the detoxification of $\mathrm{H}_{2} \mathrm{O}_{2}$ as a catalase or peroxidases. The physiological role and importance of the peroxidatic reaction in the anti-oxidant process remains unclear, in large part because the identity of the in vivo peroxidatic substrate(s) remains unknown. The wide variety of in vitro substrates that are utilized by $\mathrm{KatG}$ and the wide variety of substrates used by the closely related plant peroxidases, ranging from whole proteins to simple carbohydrates and metal ions, have not helped in the identification. Furthermore, the structures of KatGs present several potential substrate-binding sites on their surface topographys, and there is an abnormally high percentage of highly conserved residues close to the protein surface, consistent with the idea of important features residing in other than the heme pocket. Of the known substrates, $\mathrm{H}_{2} \mathrm{O}_{2}$ binds at the heme iron, which is also the most likely site for $\mathrm{O}_{2}$ binding and INH binds in the heme cavity or in the entrance channel near Ser324 (equivalent to Ser315) (Deeamgarn et al., 2005). The peroxidatic substrates probably also bind in the vicinity of Ser324 as suggested by benzhydroxamic acid binding in HRP (Henriksin et al., 1998), although the substrates used in this study are larger and would not fit into as small a cavity as benzhydroxamic 
acid. Trying to identify the NADH-binding site would be pure conjecture at this point, but the possibility that the cross-linked side chains of Trp111, Tyr238, and Met264 may have a role in electron transfer from $\mathrm{NADH}$ to $\mathrm{O}_{2}$, and the observations that INH does not inhibit $\mathrm{NADH}$ oxidation and $\mathrm{NAD}^{+}$does not inhibit the hydrazine lyase reaction suggest that the NADH-binding site is some distance from the heme pocket. However, this surmise must be tempered by the logic of having $\mathrm{NAD}^{+}$bind in close proximity to the site of isonicotinoyl radical formation. A structural definition of the binding sites is needed.

\subsection{Modulation of isoniazid activation by Ser315 variants in MtKatG}

An understanding of the role of $\mathrm{KatG}$ in the formation of isonicotinoyl-NAD has not been advanced greatly by the four native KatG structures (Yamada et al., 2002; Carpena et al., 2003; Vada et al., 2002; and Bertrand et al., 2004). One paramount question that remains unanswered is the location of the $\mathrm{INH}$ and $\mathrm{NADH} / \mathrm{NAD}^{+}$binding sites in KatG, although the location of INH binding in the structurally related horseradish peroxidase suggests a site close to the $\delta$-meso $\left(\mathrm{C}_{20}\right)$ edge of the heme (Pierattelli et al., 2004). The Ser315Thr and Ser315Asn mutations are among the commonest causes of INH resistance (Heym et al., 1995) and while retaining significant catalase and peroxidase activities the variant enzymes exhibit a reduced affinity for INH (Yu et al., 2003). Both the Ser $315 \mathrm{Thr}$ and Ser315Asn variants constructed in this study showed reduced NADH oxidase and INH hydrazine lyase activities compared to the native enzyme (Table 3.4.5), and this is mirrored in a slower rate of isonicotinoyl-NAD adduct formation by both variants (Figure 3.4.7). Recent crystallization of the Ser315Thr variant 
of MtKatG (Zhao et al., 2006) and the equivalent Ser324Thr from BpKatG (Deemagran et al., 2005) have demonstrated that replacement of Ser with Thr causes a small change in conformation of the main-chain atoms including a small displacement of the adjacent main-chain carbonyl oxygen atoms. While these appear to be very subtle changes, their effect on isonicotinoyl-NAD synthesis and INH affinity is significant as shown in this study and in a previous report (Yu et al., 2003). The proposed binding site for isoniazid lies in a cavity on the distal side of the heme close to its $\delta$-meso edge (Pierattelli et al., 2004). To reach this site in $\mathrm{KatG}$, isoniazid must pass through the channel leading to the heme cavity, passing adjacent to Ser315. The mutation of Ser315 (MtkatG) or Ser324 (BpKatG) to Thr narrows the heme access channel from around 6 to $4.7 \AA$ at its narrowest point in the mutant enzymes from both organisms (Deemagarn et al., 2005; Zhao et al., 2006). These observations in combination with significantly decreased INH activation in these variants suggest that drug binding within the heme pocket is a likely requirement for $\mathbb{N H}$ activation. Consequently, it is interference with $\mathbb{N H}$ accessibility to the heme cavity that best explains the reduced affinity and reactivity of KatG towards INH, although the possibility of $\mathrm{NHH}$ binding in the channel cannot be excluded, in which case the methyl group would interfere with $\mathbb{N H}$ binding. Other mutations of this serine residue might cause a similar blockage of the channel.

\subsection{Future directions}

Recent success in the crystallization of KatGs has excited researchers studying these enzymes. In addition, the discovery of a new activity in this well studied enzyme is both interesting and intriguing. Further characterization of the NADH oxidase reactions 
in KatGs other than those used in this study is needed. This would help in determining the physiological relevance of this activity. One of the observations during this study was variations in NADH oxidase activities from different batches of BpKatG. Different batch of EcKatG, prepared while writing this thesis, also demonstrated substantial NADH oxidase activity as compared to very low levels mentioned in this study. This raises the question about the requirement of specific conditions for expressing the enzyme and needs explanation. A recent report associating a pH induced perhydroxy modification of Trp 111 in BpKatG with NADH oxidase activity (Carpena et al., 2006) stresses the importance of crystallization of Trp111 variants of BpKatG so as to confirm the modulation of NADH oxidase activity and its association with Trp111. This study has determined the catalytic role of KatGs in isoniazid activation and isonicotinoyl-NAD synthesis over the non-enzymatic $\mathrm{Mn}^{2+}$ dependent drug activation. However, the lack of identity of the binding sites for $\mathrm{INH}$ and $\mathrm{NADH} / \mathrm{NAD}^{+}$hinders a complete understanding of how KatG's modulates NADH oxidase, hydrazine lyase and isonicotinoyl-NAD synthase activities. The identification of ligand binding sites remains the ultimate goal of future work. 


\section{REFERENCES}

Ausubel, F.M., Brent, R., Kingston, R.E., Moore, D.D., Seidman, J.G., Smith, J.A., \& Strunhl, K. (1989). Current Protocols in Molecular Biology. Green Publishing-Weiley InterScience.. New York

Ahmed, S. A. \& Claiborne, A. (1989). The streptococcal flavoprotein NADH oxidase. I. Evidence linking NADH oxidase and NADH peroxidase cysteinyl redox centers. J. Biol. Chem.264, 19856-19863.

Akazawa, T. \& Conn, E. E. (1958). The oxidation of reduced pyridine nucleotides by peroxidase. J. Biol. Chem.232, 403-415.

Allgood, G. S. \& Perry, J. J. (1986). Characterization of a manganese-containing catalase from the obligate thermophile Thermoleophilum album. J. Bacteriol. 168, 563-567.

Altamirano, M., Marostenmaki, J., Wong, A., FitzGerald, M., Black, W. A. \& Smith, J. A. (1994). Mutations in the catalase-peroxidase gene from isoniazidresistant Mycobacterium tuberculosis isolates. J. Infect. Dis. 169, 1162-1165.

Auclair, C., Torres, M., \& Hakim, J. (1978). Superoxide anion involvement in NBT reduction catalyzed by NADPH-cytochrome P-450 reductase: a pitfall. FEBS Lett. 89, 26-28

Banerjee, A., Dubnau, E., Quemard, A., Balasubramanian, V., Um, K. S., Wilson, T., Collins, D., de Lisle, G. \& Jacobs, W. R., Jr. (1994). inhA, a gene encoding a target for isoniazid and ethionamide in Mycobacterium tuberculosis. Science. 263, 227-230.

Basso, L. A., Zheng, R., Musser, J. M., Jacobs, W. R., Jr. \& Blanchard, J. S. (1998). Mechanisms of isoniazid resistance in Mycobacterium tuberculosis: enzymatic characterization of enoyl reductase mutants identified in isoniazid-resistant clinical isolates. J. Infect. Dis. 178, 769-775.

Berglund, G. I., Carlsson, G. H., Smith, A. T., Szoke, H., Henriksen, A. \& Hajdu, J. (2002). The catalytic pathway of horseradish peroxidase at high resolution. Nature. $417,463-468$.

Bertrand, T., Eady, N. A., Jones, J. N., Jesmin, Nagy, J. M., Jamart-Gregoire, B., Raven, E. L. \& Brown, K. A. (2004). Crystal structure of Mycobacterium tuberculosis catalase-peroxidase. J. Biol. Chem.279, 38991-38999.

Bravo, J., Verdaguer, N., Tormo, J., Betzel, C., Switala, J., Loewen, P. C. \& Fita, I. (1995). Crystal structure of catalase HPII from Escherichia coli. Structure 3, 491502. 
Bravo, J., Fita, I., Ferrer, J. C., Ens, W., Hillar, A., Switala, J. \& Loewen, P. C. (1997). Identification of a novel bond between a histidine and the essential tyrosine in catalase HPII of Escherichia coli. Protein Sci 6, 1016-1023.

Bravo, J., Mate, M. J., Schneider, T., Switala, J., Wilson, K., Loewen, P. C. \& Fita, I. (1999). Structure of catalase HPII from Escherichia coli at 1.9 A resolution. Proteins 34, 155-166.

Brunder, W., Schmidt, H. \& Karch, H. (1996). KatP, a novel catalase-peroxidase encoded by the large plasmid of enterohaemorrhagic Escherichia coli O157:H7. Microbiology 142 ( Pt 11), 3305-3315.

Bunkelmann, J. R. \& Trelease, R. N. (1996). Ascorbate peroxidase. A prominent membrane protein in oilseed glyoxysomes. Plant Physol.110, 589-598.

Buse, G., Soulimane, T., Dewor, M., Meyer, H. E. \& Bluggel, M. (1999). Evidence for a copper-coordinated histidine-tyrosine cross-link in the active site of cytochrome oxidase. Protein Sci 8, 985-990.

Cabiscol, E., Tamarit, J. \& Ros, J. (2000). Oxidative stress in bacteria and protein damage by reactive oxygen species. Int Microbiol 3, 3-8.

Carpena, X., Perez, R., Ochoa, W. F., Verdaguer, N., Klotz, M. G., Switala, J., Melik-Adamyan, W., Fita, I. \& Loewen, P. C. (2001). Crystallization and preliminary X-ray analysis of clade I catalases from Pseudomonas syringae and Listeria seeligeri. Acta. Crystallogr. D. Biol. Crystallogr. 57, 1184-1186.

Carpena, X., Guarne, A., Ferrer, J. C., Alzari, P. M., Fita, I. \& Loewen, P. C. (2002a). Crystallization and preliminary X-ray analysis of the hydroperoxidase I Cterminal domain from Escherichia coli. Acta. Crystallogr. D. Biol. Crystallogr. 58, 853-855.

Carpena, X., Switala, J., Loprasert, S., Mongkolsuk, S., Fita, I. \& Loewen, P. C. $(2002 b)$. Crystallization and preliminary X-ray analysis of the catalase-peroxidase KatG from Burkholderia pseudomallei. Acta. Crystallogr. D. Biol. Crystallogr. 58, 2184-2186.

Carpena, X., Loprasert, S., Mongkolsuk, S., Switala, J., Loewen, P. C. \& Fita, I. (2003a). Catalase-peroxidase $\mathrm{KatG}$ of Burkholderia pseudomallei at $1.7 \mathrm{~A}$ resolution. J. Mol. Biol. 327, 475-489.

Carpena, X., Soriano, M., Klotz, M. G., Duckworth, H. W., Donald, L. J., MelikAdamyan, W., Fita, I. \& Loewen, P. C. (2003b). Structure of the Clade 1 catalase, CatF of Pseudomonas syringae, at 1.8 A resolution. Proteins 50, 423-436. 
Carpena, X., Melik-Adamyan, W., Loewen, P. C. \& Fita, I. (2004). Structure of the C-terminal domain of the catalase-peroxidase KatG from Escherichia coli. Acta. Crystallogr. D. Biol. Crystallogr. 60, 1824-1832.

Carpena, X., Wiseman, B., Deemagarn, T., Singh, R., Switala, J., Ivancich, A., Fita, I. \& Loewen, P. C. (2005). A molecular switch and electronic circuit modulate catalase activity in catalase-peroxidases. EMBO Rep. 6, 1156-1162.

Carpena, X., Wiseman, B., Deemagarn, T., Herguedas, B., Ivancich, A., Singh, R., Loewen, P. C. \& Fita, I. (2006). Roles for Arg426 and Trp111 in the modulation of NADH oxidase activity of the catalase-peroxidase KatG from Burkholderia pseudomallei inferred from $\mathrm{pH}$-induced structural changes. Biochemistry. 45, 51715179.

Chance, B., Sies, H. \& Boveris, A. (1979). Hydroperoxide metabolism in mammalian organs. Physiol. Rev. 59, 527-605.

Chelikani, P., Carpena, X., Fita, I. \& Loewen, P. C. (2003a). An electrical potential in the access channel of catalases enhances catalysis. J. Biol. Chem.278, 31290-31296.

Chelikani, P., Donald, L. J., Duckworth, H. W. \& Loewen, P. C. (2003b). Hydroperoxidase II of Escherichia coli exhibits enhanced resistance to proteolytic cleavage compared to other catalases. Biochemistry. 42, 5729-5735.

Chelikani, P., Fita, I. \& Loewen, P. C. (2004). Diversity of structures and properties among catalases. Cell Mol. Life Sci. 61, 192-208.

Chelikani, P., Carpena, X., Perez-Luque, R., Donald, L. J., Duckworth, H. W., Switala, J., Fita, I. \& Loewen, P. C. (2005). Characterization of a large subunit catalase truncated by proteolytic cleavage. Biochemistry. 44, 5597-5605.

Chen, Y. S., Chen, S. C., Wu, T. R., Kao, C. M. \& Chen, Y. L. (2004). Seroprevalence of anti-flagellin antibody against Burkholderia pseudomalle $i$ in Taiwan. Jpn. J. Infect. Dis. 57, 224-225.

Cheng, A. C. \& Currie, B. J. (2005). Melioidosis: epidemiology, pathophysiology, and management. Clin. Microbiol. Rev. 18, 383-416.

Choi, H. J., Kang, S. W., Yang, C. H., Rhee, S. G. \& Ryu, S. E. (1998). Crystal structure of a novel human peroxidase enzyme at 2.0 A resolution. Nat. Struct. Biol. 5, 400-406.

Chouchane, S., Lippai, I. \& Magliozzo, R. S. (2000). Catalase-peroxidase (Mycobacterium tuberculosis $\mathrm{KatG}$ ) catalysis and isoniazid activation. Biochemistry. 39, 9975-9983. 
Chouchane, S., Girotto, S., Kapetanaki, S., Schelvis, J. P., Yu, S. \& Magliozzo, R. S. (2003). Analysis of heme structural heterogeneity in Mycobacterium tuberculosis catalase-peroxidase (KatG). J. Biol. Chem.278, 8154-8162.

Christman, M. F., Morgan, R. W., Jacobson, F. S. \& Ames, B. N. (1985). Positive control of a regulon for defenses against oxidative stress and some heat-shock proteins in Salmonella typhimurium. Cell 41, 753-762.

Christman, M. F., Storz, G. \& Ames, B. N. (1989). OxyR, a positive regulator of hydrogen peroxide-inducible genes in Escherichia coli and Salmonella typhimurium, is homologous to a family of bacterial regulatory proteins. Proc. Natl. Acad. Sci. US A. $86,3484-3488$.

Chung, C. T., Niemela, S. L. \& Miller, R. H. (1989). One-step preparation of competent Escherichia coli: transformation and storage of bacterial cells in the same solution. Proc. Natl. Acad. Sci. US A. 86, 2172-2175.

Claiborne, A. \& Fridovich, I. (1979a). Purification of the o-dianisidine peroxidase from Escherichia coli B. Physicochemical characterization and analysis of its dual catalatic and peroxidatic activities. J. Biol. Chem.254, 4245-4252.

Claiborne, A. \& Fridovich, I. (1979b). Chemical and enzymatic intermediates in the peroxidation of o-dianisidine by horseradish peroxidase. 2. Evidence for a substrate radical--enzyme complex and its reaction with nucleophiles. Biochemistry. 18, 23292335 .

Claiborne, A. \& Fridovich, I. (1979c). Chemical and enzymatic intermediates in the peroxidation of o-dianisidine by horseradish peroxidase. 1. Spectral properties of the products of dianisidine oxidation. Biochemistry. 18, 2324-2329.

Clare, D. A., Duong, M. N., Darr, D., Archibald, F. \& Fridovich, I. (1984). Effects of molecular oxygen on detection of superoxide radical with nitroblue tetrazolium and on activity stains for catalase. Anal. Biochem. 140, 532-537.

Corbett, E. L., Charalambous, S., Fielding, K., Clayton, T., Hayes, R. J., De Cock, K. M. \& Churchyard, G. J. (2003). Stable incidence rates of tuberculosis (TB) among human immunodeficiency virus (HIV)-negative South African gold miners during a decade of epidemic HIV-associated TB. J. Infect. Dis. s 188, $1156-$ 1163.

Cypionka, H. (2000). Oxygen respiration by Desulfovibrio species. Annu. Rev. Microbiol. 54, 827-848. 
Dang, P. M., Babior, B. M. \& Smith, R. M. (1999). NADPH dehydrogenase activity of p67PHOX, a cytosolic subunit of the leukocyte NADPH oxidase. Biochemistry. 38, 5746-5753.

Dawson, J. H., Bracete, A. M., Huff, A. M., Kadkhodayan, S., Zeitler, C. M., Sono, M., Chang, C. K. \& Loewen, P. C. (1991). The active site structure of E. coli HPII catalase. Evidence favoring coordination of a tyrosinate proximal ligand to the chlorin iron. FEBS Lett. 295, 123-126.

Deemagarn, T., Carpena, X., Singh, R., Wiseman, B., Fita, I. \& Loewen, P. C. (2005). Structural characterization of the Ser324Thr variant of the catalase-peroxidase (KatG) from Burkholderia pseudomallei. J. Mol. Biol. 345, 21-28.

Demple, B. \& Harrison, L. (1994). Repair of oxidative damage to DNA: enzymology and biology. Annu. Rev. Biochem. 63, 915-948.

Deretic, V., Philipp, W., Dhandayuthapani, S., Mudd, M. H., Curcic, R., Garbe, T., Heym, B., Via, L. E. \& Cole, S. T. (1995). Mycobacterium tuberculosis is a natural mutant with an inactivated oxidative-stress regulatory gene: implications for sensitivity to isoniazid. Mol. Microbiol. 17, 889-900.

Deretic, V., Pagan-Ramos, E., Zhang, Y., Dhandayuthapani, S. \& Via, L. E. (1996). The extreme sensitivity of Mycobacterium tuberculosis to the front-line antituberculosis drug isoniazid. Nat. Biotechnol. 14, 1557-1561.

Dhandayuthapani, S., Mudd, M. \& Deretic, V. (1997). Interactions of OxyR with the promoter region of the $\operatorname{xyy} R$ and $a h p C$ genes from Mycobacterium leprae and Mycobacterium tuberculosis. J. Bacteriol. 179, 2401-2409.

Diaz, A., Horjales, E., Rudino-Pinera, E., Arreola, R. \& Hansberg, W. (2004). Unusual Cys-Tyr covalent bond in a large catalase. J. Mol. Biol. 342, 971-985.

Diaz, G. A. \& Wayne, L. G. (1974). Isolation and characterization of catalase produced by Mycobacterium tuberculosis. Am. Rev. Respir. Dis. 110, 312-319.

Donald, L. J., Krokhin, O. V., Duckworth, H. W., Wiseman, B., Deemagran, T., Singh, R., Switala, J., Carpena, X., Fita, I., Loewen, P.C. (2003). Characterization of the catalase-peroxidase KatG from Burkholderia pseudomalle $i$ by mass spectrometry. J. Biol. Chem.278, 35687-35692.

Doroshow, J. H., Leong, L., Margolin, K., Flanagan, B., Golberg, D., Akman, S., Carr, B., Odujinrin, O., Newman, E. (1989). Refractory metastatic breast cancer: salvage therapy with fluorouracil and high-dose continuous infusion leucovorin calcium. J. Clin. Oncol. 7, 439-444. 
Dos Santos, D. M. \& Berridge, D. M. (2000). A continuation ratio random effects model for repeated ordinal responses. Stat. Med. 19, 3377-3388.

Dunford, H. B. (1999) Heme peroxidases. Wiley-VCH. New York

Dunford, H. B. \& Hsuanyu, Y. (1999). Kinetics of oxidation of serotonin by myeloperoxidase compounds I and II. Biochem. Cell. Biol. 77, 449-457.

Eiglmeier, K., Fsihi, H., Heym, B. \& Cole, S. T. (1997). On the catalase-peroxidase gene, $k a t G$, of Mycobacterium leprae and the implications for treatment of leprosy with isoniazid. FEMS Microbiol. Lett. 149, 273-278.

Escolar, L., Perez-Martin, J. \& de Lorenzo, V. (1999). Opening the iron box: transcriptional metalloregulation by the Fur protein. J. Bacteriol. 181, 6223-6229.

Faguy, D. M. (2000). The controlled chaos of shifty pathogens. Curr. Biol. 10, R498501.

Faguy, D. M. \& Doolittle, W. F. (2000). Horizontal transfer of catalase-peroxidase genes between archaea and pathogenic bacteria. Trends. Genet. 16, 196-197.

Feldman, E. L., Stevens, M. J. \& Greene, D. A. (1997). Pathogenesis of diabetic neuropathy. Clin. Neurosci.4, 365-370.

Filizola, M., Laakkonen, L. \& Loew, G. H. (1999). 3D modeling, ligand binding and activation studies of the cloned mouse delta, mu; and kappa opioid receptors. Protein. Eng. 12, 927-942.

Fita, I. \& Rossmann, M. G. (1985). The active center of catalase. J. Mol. Biol. 185, $21-37$.

Flohe, L., Gunzler, W. A. \& Schock, H. H. (1973). Glutathione peroxidase: a selenoenzyme. FEBS Lett. 32, 132-134.

Flohe, L. (1988). Glutathione peroxidase. Basic Life Sci. 49, 663-668.

Forkl, H., Vandekerckhove, J., Drews, G. \& Tadros, M. H. (1993). Molecular cloning, sequence analysis and expression of the gene for catalase-peroxidase (cpeA) from the photosynthetic bacterium Rhodobacter capsulatus B10. Eur. J. Biochem. $214,251-258$.

Fridovich, I. (1975a). Superoxide dismutases. Annu. Rev. Biochem. . 44, 147-159.

Fridovich, I. (1975b). Oxygen: boon and bane. Am. Sci. 63, 54-59. 
Fridovich, I. (1978). Superoxide dismutases: defence against endogenous superoxide radical. Ciba. Found. Symp., 77-93.

Fridovich, I. (1995). Superoxide radical and superoxide dismutases. Annu. Rev. Biochem. 64, 97-112.

Fridovich, I. (1997). Superoxide anion radical (O2-), superoxide dismutases, and related matters. J. Biol. Chem.272, 18515-18517.

Fridovich, I. (1999). Fundamental aspects of reactive oxygen species, or what's the matter with oxygen? Ann. N. Y. Acad. Sci. 893, 13-18.

Gardner, T. J. (1988). Oxygen radicals in cardiac surgery. Free. Radic. Biol. Med. 4, 45-50.

Garnier, T., Eiglmeier, K., Camus, J. C. \& other authors (2003). The complete genome sequence of Mycobacterium bovis. Proc. Natl. Acad. Sci. US A. 100,78777882 .

Ghiladi, R. A., Knudsen, G. M., Medzihradszky, K. F. \& Ortiz de Montellano, P. R. (2005a). The Met-Tyr-Trp cross-link in Mycobacterium tuberculosis catalaseperoxidase (KatG): autocatalytic formation and effect on enzyme catalysis and spectroscopic properties. J. Biol. Chem.280, 22651-22663.

Ghiladi, R. A., Medzihradszky, K. F. \& Ortiz de Montellano, P. R. (2005b). Role of the Met-Tyr-Trp cross-link in Mycobacterium tuberculosis catalase-peroxidase (KatG) as revealed by KatG(M255I). Biochemistry. 44, 15093-15105.

Ghiladi, R. A., Medzihradszky, K. F., Rusnak, F. M. \& Ortiz de Montellano, P. R. (2005c). Correlation between isoniazid resistance and superoxide reactivity in Mycobacterium tuberculosis KatG. J. Am. Chem. Soc. 127, 13428-13442.

Gouet, P., Jouve, H. M. \& Dideberg, O. (1995). Crystal structure of Proteus mirabilis PR catalase with and without bound NADPH. J. Mol. Biol. 249, 933-954.

Gray, H. B. \& Winkler, J. R. (1996). Electron transfer in proteins. Annu. Rev. Biochem. 65, 537-561.

Gray, H. B. \& Winkler, J. R. (2003). Electron tunneling through proteins. Q. Rev. Biophys. 36, 341-372.

Gregory, E. M. \& Fridovich, I. (1974). Visualization of catalase on acrylamide gels. Anal. Biochem. 58, 57-62. 
Gutierrez, M. C., Brisse, S., Brosch, R., Fabre, M., Omais, B., Marmiesse, M., Supply, P. \& Vincent, V. (2005). Ancient origin and gene mosaicism of the progenitor of Mycobacterium tuberculosis. PLoS Pathog. 1, e5.

Gutteridge, J. M. \& Mitchell, J. (1999). Redox imbalance in the critically ill. Br. Med. Bull. 55, 49-75.

Harman, L. S., Carver, D. K., Schreiber, J. \& Mason, R. P. (1986). One- and twoelectron oxidation of reduced glutathione by peroxidases. J. Biol. Chem.261, 16421648 .

Hatchikian, E. C. \& Henry, Y. A. (1977). An iron-containing superoxide dismutase from the strict anaerobe Desulfovibrio desulfuricans (Norway 4). Biochimie. 59, 153161.

Heering, H. A., Indiani, C., Regelsberger, G., Jakopitsch, C., Obinger, C. \& Smulevich, G. (2002). New insights into the heme cavity structure of catalaseperoxidase: a spectroscopic approach to the recombinant Synechocystis enzyme and selected distal cavity mutants. Biochemistry. 41, 9237-9247.

Heym, B., Alzari, P. M., Honore, N. \& Cole, S. T. (1995). Missense mutations in the catalase-peroxidase gene, $k a t \mathrm{G}$, are associated with isoniazid resistance in Mycobacterium tuberculosis. Mol. Microbiol. 15, 235-245.

Heym, B., Philipp, W. \& Cole, S. T. (1996). Mechanisms of drug resistance in Mycobacterium tuberculosis. Curr. Top. Microbiol. Immunol. 215, 49-69.

Hillar, A., Nicholls, P., Switala, J. \& Loewen, P. C. (1994). NADPH binding and control of catalase compound II formation: comparison of bovine, yeast, and Escherichia coli enzymes. Biochem. J. 300 ( Pt 2), 531-539.

Hillar, A. \& Loewen, P. C. (1995). Comparison of isoniazid oxidation catalyzed by bacterial catalase-peroxidases and horseradish peroxidase. Arch. Biochem. Biophys. $323,438-446$.

Hillar, A., Van Caeseele, L. \& Loewen, P. C. (1999). Intracellular location of catalase-peroxidase hydroperoxidase I of Escherichia coli. FEMS Microbiol. Lett. $170,307-312$.

Hillar, A., Peters, B., Pauls, R., Loboda, A., Zhang, H., Mauk, A. G. \& Loewen, P. C. (2000). Modulation of the activities of catalase-peroxidase HPI of Escherichia coli by site-directed mutagenesis. Biochemistry. 39, 5868-5875.

Hochman, A. \& Goldberg, I. (1991). Purification and characterization of a catalaseperoxidase and a typical catalase from the bacterium Klebsiella pneumoniae.

Biochim. Biophys. Acta. 1077, 299-307. 
Horsburgh, M. J., Clements, M. O., Crossley, H., Ingham, E. \& Foster, S. J. (2001a). PerR controls oxidative stress resistance and iron storage proteins and is required for virulence in Staphylococcus aureus. Infect. Immun. 69, 3744-3754.

Horsburgh, M. J., Ingham, E. \& Foster, S. J. (2001b). In Staphylococcus aureus, fur is an interactive regulator with PerR, contributes to virulence, and is necessary for oxidative stress resistance through positive regulation of catalase and iron homeostasis. J. Bacteriol. 183, 468-475.

Imlay, J. A. \& Fridovich, I. (1991a). Assay of metabolic superoxide production in Escherichia coli. J. Biol. Chem.266, 6957-6965.

Imlay, J. A. \& Fridovich, I. (1991b). Superoxide production by respiring membranes of Escherichia coli. Free. Radic. Res. Commun. 12-13 Pt 1, 59-66.

Imlay, K. R. \& Imlay, J. A. (1996). Cloning and analysis of sodC, encoding the copper-zinc superoxide dismutase of Escherichia coli. J. Bacteriol. 178, 2564-2571.

Ito, N., Phillips, S. E., Yadav, K. D. \& Knowles, P. F. (1994). Crystal structure of a free radical enzyme, galactose oxidase. J. Mol. Biol. 238, 794-814.

Itoh, S., Takayama, S., Arakawa, R., Furuta, A., Komatsu, M., Ishida, A., Takamuku, S. \& Fukuzumi, S. (1997). Active site models for galactose oxidase. Electronic fffect of the thioether group in the novel organic cofactor. Inorg. Chem. 36, 1407-1416.

Ivancich, A., Lutz, M. \& Mattioli, T. A. (1997). Temperature-dependent behavior of bacteriochlorophyll and bacteriopheophytin in the photosynthetic reaction center from Rhodobacter sphaeroides. Biochemistry. 36, 3242-3253.

Ivancich, A., Artz, K., Williams, J. C., Allen, J. P. \& Mattioli, T. A. (1998). Effects of hydrogen bonds on the redox potential and electronic structure of the bacterial primary electron donor. Biochemistry. 37, 11812-11820.

Ivancich, A., Mazza, G. \& Desbois, A. (2001). Comparative electron paramagnetic resonance study of radical intermediates in turnip peroxidase isozymes. Biochemistry. 40, 6860-6866.

Ivancich, A., Jakopitsch, C., Auer, M., Un, S. \& Obinger, C. (2003). Protein-based radicals in the catalase-peroxidase of Synechocystis PCC6803: a multifrequency EPR investigation of wild-type and variants on the environment of the heme active site. $J$. Am. Chem. Soc. 125, 14093-14102.

Jakopitsch, C., Ruker, F., Regelsberger, G., Dockal, M., Peschek, G. A. \& Obinger, C. (1999). Catalase-peroxidase from the cyanobacterium Synechocystis 
PCC 6803: cloning, overexpression in Escherichia coli, and kinetic characterization. Biol. Chem. 380, 1087-1096.

Jakopitsch, C., Regelsberger, G., Furtmuller, P. G., Ruker, F., Peschek, G. A. \& Obinger, C. (2001). Catalase-peroxidase from Synechocystis is capable of chlorination and bromination reactions. Biochem. Biophys. Res. Commun. 287, 682687.

Jakopitsch, C., Auer, M., Ivancich, A., Ruker, F., Furtmuller, P. G. \& Obinger, C. (2003a). Total conversion of bifunctional catalase-peroxidase (KatG) to monofunctional peroxidase by exchange of a conserved distal side tyrosine. J. Biol. Chem.278, 20185-20191.

Jakopitsch, C., Auer, M., Regelsberger, G., Jantschko, W., Furtmuller, P. G., Ruker, F. \& Obinger, C. (2003b). The catalytic role of the distal site asparaginehistidine couple in catalase-peroxidases. Eur. J. Biochem. / FEBS 270, 1006-1013.

Jakopitsch, C., Auer, M., Regelsberger, G., Jantschko, W., Furtmuller, P. G., Ruker, F. \& Obinger, C. (2003c). Distal site aspartate is essential in the catalase activity of catalase-peroxidases. Biochemistry. 42, 5292-5300.

Jakopitsch, C., Kolarich, D., Petutschnig, G., Furtmuller, P. G. \& Obinger, C. (2003d). Distal side tryptophan, tyrosine and methionine in catalase-peroxidases are covalently linked in solution. FEBS Lett. 552, 135-140.

Jakopitsch, C., Ivancich, A., Schmuckenschlager, F., Wanasinghe, A., Poltl, G., Furtmuller, P. G., Ruker, F. \& Obinger, C. (2004). Influence of the unusual covalent adduct on the kinetics and formation of radical intermediates in Synechocystis catalase peroxidase: a stopped-flow and EPR characterization of the MET275, TYR249, and ARG439 variants. J. Biol. Chem.279, 46082-46095.

Jakopitsch, C., Droghetti, E., Schmuckenschlager, F., Furtmuller, P. G., Smulevich, G. \& Obinger, C. (2005a). Role of the main access channel of catalaseperoxidase in catalysis. J. Biol. Chem.280, 42411-42422.

Jakopitsch, C., Wanasinghe, A., Jantschko, W., Furtmuller, P. G. \& Obinger, C. (2005b). Kinetics of interconversion of ferrous enzymes, compound II and compound III, of wild-type Synechocystis catalase-peroxidase and Y249F: proposal for the catalatic mechanism. J. Biol. Chem.280, 9037-9042.

Jenney, F. E., Jr., Verhagen, M. F., Cui, X. \& Adams, M. W. (1999). Anaerobic microbes: oxygen detoxification without superoxide dismutase. Science. 286, $306-$ 309. 
Johnsson, K., Froland, W. A. \& Schultz, P. G. (1997). Overexpression, purification, and characterization of the catalase-peroxidase $\mathrm{Kat} G$ from Mycobacterium tuberculosis. J. Biol. Chem.272, 2834-2840.

Jovanovic, T., Ascenso, C., Hazlett, K. R. \& other authors (2000). Neelaredoxin, an iron-binding protein from the syphilis spirochete, Treponema pallidum, is a superoxide reductase. J. Biol. Chem. 275, 28439-28448.

Jung, I. L. \& Kim, I. G. (2003). Transcription of ahpC, katG, and katE genes in Escherichia coli is regulated by polyamines: polyamine-deficient mutant sensitive to $\mathrm{H}_{2} \mathrm{O}_{2}$ induced oxidative damage. Biochem. Biophys. Res. Commun. 301, 915-922.

Kalko, S. G., Gelpi, J. L., Fita, I. \& Orozco, M. (2001). Theoretical study of the mechanisms of substrate recognition by catalase. J. Am. Chem. Soc. 123, 9665-9672.

Kao, S. M. \& Hassan, H. M. (1985). Biochemical characterization of a paraquattolerant mutant of Escherichia coli. J. Biol. Chem.260, 10478-10481.

Kapetanaki, S. M., Chouchane, S., Yu, S., Magliozzo, R. S. \& Schelvis, J. P. (2005). Resonance Raman spectroscopy of Compound II and its decay in Mycobacterium tuberculosis catalase-peroxidase $\mathrm{KatG}$ and its isoniazid resistant mutant S315T. Journal of inorganic Biochemistry. 99, 1401-1406.

Kapur, V., Li, L. L., Hamrick, M. R. \& other authors (1995). Rapid Mycobacterium species assignment and unambiguous identification of mutations associated with antimicrobial resistance in Mycobacterium tuberculosis by automated DNA sequencing. Arch Pathol Lab Med 119, 131-138.

Kelly, P. M. (1997). Wrong drug used for tuberculosis. Int. J. Tuberc. Lung Dis. 1, 385.

Kengen, S. W., Bikker, F. J., Hagen, W. R., de Vos, W. M. \& van der Oost, J. (2001). Characterization of a catalase-peroxidase from the hyperthermophilic archaeon Archaeoglobus fulgidus. Extremophiles 5, 323-332.

Kengen, S. W., van der Oost, J. \& de Vos, W. M. (2003). Molecular characterization of $\mathrm{H}_{2} \mathrm{O}_{2}$ forming NADH oxidases from Archaeoglobus fulgidus. Eur. J. Biochem. 270, 2885-2894.

Kespichayawattana, W., Rattanachetkul, S., Wanun, T., Utaisincharoen, P. \& Sirisinha, S. (2000). Burkholderia pseudomallei induces cell fusion and actinassociated membrane protrusion: a possible mechanism for cell-to-cell spreading. Infect. Immun. 68, 5377-5384. 
Klenk, H. P., Clayton, R. A., Tomb, J. F. \& other authors (1997). The complete genome sequence of the hyperthermophilic, sulphate-reducing archaeon Archaeoglobus fulgidus. Nature. 390, 364-370.

Klotz, M. G., Klassen, G. R. \& Loewen, P. C. (1997). Phylogenetic relationships among prokaryotic and eukaryotic catalases. Mol. Biol. Evol. 14, 951-958.

Klotz, M. G. \& Loewen, P. C. (2003). The molecular evolution of catalatic hydroperoxidases: evidence for multiple lateral transfer of genes between prokaryota and from bacteria into eukaryota. Mol. Biol. Evol. 20, 1098-1112.

Ko, T. P., Day, J., Malkin, A. J. \& McPherson, A. (1999). Structure of orthorhombic crystals of beef liver catalase. Acta. Crystallogr. D. Biol. Crystallogr. 55, 1383-1394.

Kono, Y. \& Fridovich, I. (1983). Functional significance of manganese catalase in Lactobacillus plantarum. J. Bacteriol. 155, 742-746.

Kunkel, T. A., Roberts, J. D. \& Zakour, R. A. (1987). Rapid and efficient sitespecific mutagenesis without phenotypic selection. Methods Enzymol. 154, 367-382.

Larsen, M. H., Vilcheze, C., Kremer, L. \& other authors (2002). Overexpression of inhA, but not kasA, confers resistance to isoniazid and ethionamide in Mycobacterium smegmatis, M. bovis BCG and M. tuberculosis. Mol. Microbiol. 46, 453-466.

Layne, E. (1957). Spectrophotometric and turbidimetric methods for measuring proteins. Methods Enzymol. . 3, 447-454

Lee, A. S., Tang, L. L., Lim, I. H., Ling, M. L., Tay, L. \& Wong, S. Y. (1997). Lack of clinical significance for the common arginine-to-leucine substitution at codon 463 of the kat $G$ gene in isoniazid-resistant Mycobacterium tuberculosis in Singapore. J. Infect. Dis 176, 1125-1127.

Leelarasamee, A. (2004). Recent development in melioidosis. Curr. Opin. Infect. Dis. 17, 131-136.

Lei, B., Wei, C. J. \& Tu, S. C. (2000). Action mechanism of antitubercular isoniazid. Activation by Mycobacterium tuberculosis $\mathrm{KatG}$, isolation, and characterization of inhA inhibitor. J. Biol. Chem.275, 2520-2526.

Li, L., Bannantine, J. P., Zhang, Q., Amonsin, A., May, B. J., Alt, D., Banerji, N., Kanjilal, S. \& Kapur, V. (2005). The complete genome sequence of Mycobacterium avium subspecies paratuberculosis. Proc. Natl. Acad. Sci. USA. 102, 12344-12349. 
Liochev, S. I. \& Fridovich, I. (1992). Superoxide generated by glutathione reductase initiates a vanadate-dependent free radical chain oxidation of NADH. Arch. Biochem. Biophys. 294, 403-406.

Liochev, S. I. \& Fridovich, I. (1997). How does superoxide dismutase protect against tumor necrosis factor: a hypothesis informed by effect of superoxide on "free" iron. Free. Radic. Biol. Med. 23, 668-671.

Loewen, P. (1996). Probing the structure of catalase HPII of Escherichia coli--a review. Gene. 179, 39-44.

Loewen, P. C., Triggs, B. L., Klassen, G. R. \& Weiner, J. H. (1983). Identification and physical characterization of a Col E1 hybrid plasmid containing a catalase gene of Escherichia coli. Can. J. Biochem. Cell Biol. 61, 1315-1321.

Loewen, P. C. (1984). Isolation of catalase-deficient Escherichia coli mutants and genetic mapping of kat E, a locus that affects catalase activity. $J$. Bacteriol. 157, 622626.

Loewen, P. C. \& Triggs, B. L. (1984). Genetic mapping of $k a t F$, a locus that with $k a t E$ affects the synthesis of a second catalase species in Escherichia coli. J. Bacteriol. 160, 668-675.

Loewen, P. C., Switala, J. \& Triggs-Raine, B. L. (1985a). Catalases HPI and HPII in Escherichia coli are induced independently. Arch. Biochem. Biophys. 243, $144-$ 149.

Loewen, P. C., Triggs, B. L., George, C. S. \& Hrabarchuk, B. E. (1985b). Genetic mapping of $k a t G$, a locus that affects synthesis of the bifunctional catalase-peroxidase hydroperoxidase I in Escherichia coli. J. Bacteriol. 162, 661-667.

Loewen, P. C. \& Switala, J. (1986). Purification and characterization of catalase HPII from Escherichia coli K12. Biochem. Cell Biol. 64, 638-646.

Loewen, P. C. \& Switala, J. (1987a). genetic mapping of katA, a locus that affects catalase 1 level in Bacillus subtilis. J. Bacteriol. 169, 5848-5851.

Loewen, P. C. \& Switala, J. (1987b). Purification and characterization of catalase-1 from Bacillus subtilis. Biochem. Cell Biol. 65, 939-947.

Loewen, P. C. \& Switala, J. (1988). Purification and characterization of sporespecific catalase-2 from Bacillus subtilis. Biochem. Cell Biol. 66, 707-714.

Loewen, P. C. (1989). genetic mapping of $k a t B$, a locus that affects catalase 2 levels in Bacillus subtilis. Can. J. Microbiol. 35, 807-810. 
Loewen, P. C., Switala, J., von Ossowski, I., Hillar, A., Christie, A., Tattrie, B. \& Nicholls, P. (1993). Catalase HPII of Escherichia coli catalyzes the conversion of protoheme to cis-heme d. Biochemistry. 32, 10159-10164.

Loewen, P. C., Carpena, X., Rovira, C., Ivancich, A., Perez-Luque, R., Haas, R., Odenbreit, S., Nicholls, P. \& Fita, I. (2004). Structure of Helicobacter pylori catalase, with and without formic acid bound, at $1.6 \AA$ resolution. Biochemistry. 43, 3089-3103.

Lombard, M., Fontecave, M., Touati, D. \& Niviere, V. (2000a). Reaction of the desulfoferrodoxin from Desulfoarculus baarsii with superoxide anion. Evidence for a superoxide reductase activity. J. Biol. Chem.275, 115-121.

Lombard, M., Touati, D., Fontecave, M. \& Niviere, V. (2000b). Superoxide reductase as a unique defense system against superoxide stress in the microaerophile Treponema pallidum. J. Biol. Chem.275, 27021-27026.

Loprasert, S., Atichartpongkun, S., Whangsuk, W. \& Mongkolsuk, S. (1997). Isolation and analysis of the Xanthomonas alkyl hydroperoxide reductase gene and

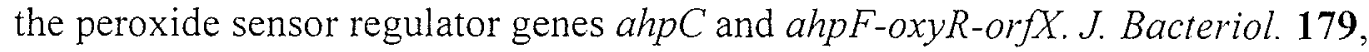
3944-3949.

Loprasert, S., Sallabhan, R., Whangsuk, W. \& Mongkolsuk, S. (2002). The Burkholderia pseudomallei oxy $R$ gene: expression analysis and mutant characterization. Gene. 296, 161-169.

Loprasert, S., Sallabhan, R., Whangsuk, W. \& Mongkolsuk, S. (2003a). Compensatory increase in $a h p C$ gene expression and its role in protecting Burkholderia pseudomallei against reactive nitrogen intermediates. Arch. Microbiol. 180, 498-502.

Loprasert, S., Whangsuk, W., Sallabhan, R. \& Mongkolsuk, S. (2003b). Regulation of the kat $G$-dpsA operon and the importance of $\mathrm{KatG}$ in survival of Burkholderia pseudomallei exposed to oxidative stress. FEBS Lett. 542, 17-21.

Loprasert, S., Whangsuk, W., Sallabhan, R. \& Mongkolsuk, S. (2004). DpsA protects the human pathogen Burkholderia pseudomallei against organic hydroperoxide. Arch. Microbiol. 182, 96-101.

Lundrigan, M. D., Arceneaux, J. E., Zhu, W. \& Byers, B. R. (1997). Enhanced hydrogen peroxide sensitivity and altered stress protein expression in iron-starved Mycobacterium smegmatis. Biometals 10,215-225.

Magliozzo, R. S. \& Marcinkeviciene, J. A. (1997). The role of Mn(II)-peroxidase activity of mycobacterial catalase-peroxidase in activation of the antibiotic isoniazid. J. Biol. Chem.272, 8867-8870. 
Maj, M., Nicholls, P., Obinger, C., Hillar, A. \& Loewen, P. C. (1996). Reaction of E. coli catalase HPII with cyanide as ligand and as inhibitor. Biochim. Biophys. Acta. 1298, 241-249.

Marcinkeviciene, J. A., Magliozzo, R. S. \& Blanchard, J. S. (1995). Purification and characterization of the Mycobacterium smegmatis catalase-peroxidase involved in isoniazid activation. J. Biol. Chem.270, 22290-22295.

Marttila, H. J., Soini, H., Huovinen, P. \& Viljanen, M. K. (1996). kat G mutations in isoniazid-resistant Mycobacterium tuberculosis isolates recovered from Finnish patients. Antimicrob. Agents. Chemother. 40, 2187-2189.

Marttila, H. J., Soini, H., Eerola, E., Vyshnevskaya, E., Vyshnevskiy, B. I., Otten, T. F., Vasilyef, A. V. \& Viljanen, M. K. (1998). A Ser315Thr substitution in $\mathrm{KatG}$ is predominant in genetically heterogeneous multidrug-resistant Mycobacterium tuberculosis isolates originating from the St. Petersburg area in Russia. Antimicrob. Agents. Chemother. 42, 2443-2445.

McCord, J. M. \& Fridovich, I. (1969a). The utility of superoxide dismutase in studying free radical reactions. I. Radicals generated by the interaction of sulfite, dimethyl sulfoxide, and oxygen. J. Biol. Chem.244, 6056-6063.

McCord, J. M. \& Fridovich, I. (1969b). Superoxide dismutase. An enzymic function for erythrocuprein (hemocuprein). J. Biol. Chem.244, 6049-6055.

Mdluli, K., Slayden, R. A., Zhu, Y., Ramaswamy, S., Pan, X., Mead, D., Crane, D. D., Musser, J. M. \& Barry, C. E., 3rd (1998a). Inhibition of a Mycobacterium tuberculosis beta-ketoacyl ACP synthase by isoniazid. Science. 280, 1607-1610.

Mdluli, K., Swanson, J., Fischer, E., Lee, R. E. \& Barry, C. E., 3rd (1998b). Mechanisms involved in the intrinsic isoniazid resistance of Mycobacterium avium. Mol. Microbiol. 27, 1223-1233.

Menendez, M. C., Ainsa, J. A., Martin, C. \& Garcia, M. J. (1997). katGI and katGII encode two different catalases-peroxidases in Mycobacterium fortuitum. $J$. Bacteriol. 179, 6880-6886.

Messerschmidt, A. \& Wever, R. (1996). X-ray structure of a vanadium-containing enzyme: chloroperoxidase from the fungus Curvularia inaequalis. Proc. Natl. Acad. Sci. USA. 93, 392-396.

Messner, K. R. \& Imlay, J. A. (2002). In vitro quantitation of biological superoxide and hydrogen peroxide generation. Methods Enzymol. 349, 354-361. 
Middlebrook, G. (1952). Sterilization of tubercle bacilli by isonicotinic acid hydrazide and the incidence of variants resistant to the drug in vitro. Am. Rev. Tuberc. 65, 765-767.

Middlebrook, G. (1954a). Techniques of drug-resistance tests. Am. Rev. Tuberc. 70, 922-923.

Middlebrook, G. (1954b). Isoniazid-resistance and catalase activity of tubercle bacilli; a preliminary report. Am. Rev. Tuberc. 69, 471-472.

Middlebrook, G., Cohn, M. L. \& Schaefer, W. B. (1954a). Studies on isoniazid and tubercle bacilli. III. The isolation, drug-susceptibility, and catalase-testing of tubercle bacilli from isoniazid-treated patients. Am. Rev. Tuberc. 70, 852-872.

Middlebrook, G., Cohn, M. L., Schaefer, W. B. \& Kovitz, C. (1954b). Developments in the laboratory: the growth requirements and pathogenic properties of isoniazid-resistant tubercle bacilli. Trans. Annu. Meet. Natl. Tuberc. Assoc. 96, 239-240.

Middlebrook, G. \& Dressler, S. H. (1954). Clinical evaluation of isoniazid. Am. Rev. Tuberc. 70, 1102-1103.

Millett, F., Miller, M. A., Geren, L. \& Durham, B. (1995). Electron transfer between cytochrome $c$ and cytochrome $c$ peroxidase. J. Bioenerg. Biomembr. 27, 341-351.

Mitchison, D. A. \& Selkon, J. B. (1956). The bactericidal activities of antituberculous drugs. Am. Rev. Tuberc. 74, 109-116; discussion, 116-123.

Morris, S., Bai, G. H., Suffys, P., Portillo-Gomez, L., Fairchok, M. \& Rouse, D. (1995). Molecular mechanisms of multiple drug resistance in clinical isolates of Mycobacterium tuberculosis. J. Infect. Dis. s 171, 954-960.

Morris, S. L., Nair, J. \& Rouse, D. A. (1992). The catalase-peroxidase of Mycobacterium intracellulare: nucleotide sequence analysis and expression in Escherichia coli. J. Gen. Microbiol. 138, 2363-2370.

Mukhopadhyay, S. \& Schellhorn, H. E. (1994). Induction of Escherichia coli hydroperoxidase I by acetate and other weak acids. J. Bacteriol. 176, 2300-2307.

Mulvey, M. R., Sorby, P. A., Triggs-Raine, B. L. \& Loewen, P. C. (1988). Cloning and physical characterization of $k a t E$ and $k a t F$ required for catalase HPII expression in Escherichia coli. Gene. 73, 337-345.

Mulvey, M. R., Switala, J., Borys, A. \& Loewen, P. C. (1990). Regulation of transcription of katE and katF in Escherichia coli. J. Bacteriol. 172, 6713-6720. 
Murshudov, G. N., Melik-Adamyan, W. R., Grebenko, A. I., Barynin, V. V., Vagin, A. A., Vainshtein, B. K., Dauter, Z. \& Wilson, K. S. (1992). Threedimensional structure of catalase from Micrococcus lysodeikticus at $1.5 \AA$ resolution. FEBS Lett. 312, 127-131.

Murshudov, G. N., Grebenko, A. I., Barynin, V. \& other authors (1996). Structure of the heme d of Penicillium vitale and Escherichia coli catalases. J. Biol. Chem.271, 8863-8868.

Murthy, M. R., Reid, T. J., 3rd, Sicignano, A., Tanaka, N. \& Rossmann, M. G. (1981). Structure of beef liver catalase. J. Mol. Biol. 152, 465-499.

Musser, J. M. (1995). Antimicrobial agent resistance in mycobacteria: molecular genetic insights. Clin. Microbiol. Rev. 8, 496-514.

Musser, J. M., Kapur, V., Williams, D. L., Kreiswirth, B. N., van Soolingen, D. \& van Embden, J. D. (1996). Characterization of the catalase-peroxidase gene (kat $G$ ) and inhA locus in isoniazid-resistant and -susceptible strains of Mycobacterium tuberculosis by automated DNA sequencing: restricted array of mutations associated with drug resistance. J. Infect. Dis 173, 196-202.

Mutsuda, M., Ishikawa, T., Takeda, T. \& Shigeoka, S. (1996). The catalaseperoxidase of Synechococcus PCC 7942: purification, nucleotide sequence analysis and expression in Escherichia coli. Biochem. J. 316 ( Pt 1), 251-257.

Nagy, J. M., Cass, A. E. \& Brown, K. A. (1995). Progress in the characterization of catalase-peroxidase from Mycobacterium tuberculosis. Biochem. Soc. Trans. 23, $152 \mathrm{~S}$.

Nagy, J. M., Cass, A. E. \& Brown, K. A. (1997). Purification and characterization of recombinant catalase-peroxidase, which confers isoniazid sensitivity in Mycobacterium tuberculosis. J. Biol. Chem.272, 31265-31271.

Nagy, J. M., Jesmin, J., Servos, S., Cass, A. E. \& Brown, K. A. (1998). Sitedirected mutants of the catalase-peroxidase from Mycobacterium tuberculosis. Biochem. Soc. Trans. 26, S281.

Nicholls, P. (1962). The role of the protein in haem enzymes. Biochim. Biophys. Acta. 60, 217-225.

Obinger, C., Maj, M., Nicholls, P. \& Loewen, P. (1997a). Activity, peroxide compound formation, and heme d synthesis in Escherichia coli HPII catalase. Arch. Biochem. Biophys. 342, 58-67. 
Obinger, C., Regelsberger, G., Strasser, G., Burner, U. \& Peschek, G. A. (1997b). Purification and characterization of a homodimeric catalase-peroxidase from the cyanobacterium Anacystis nidulans. Biochem. Biophys. Res. Commun. 235, 545552 .

Obinger, C., Regelsberger, G., Furtmuller, P. G., Jakopitsch, C., Ruker, F., Pircher, A. \& Peschek, G. A. (1999). Catalase-peroxidases in cyanobacteria-similarities and differences to ascorbate peroxidases. Free Radic. Res. $31 \mathrm{Suppl}$, S243-249.

Ostermeier, C., Harrenga, A., Ermler, U. \& Michel, H. (1997). Structure at 2.7 A resolution of the Paracoccus denitrificans two-subunit cytochrome $c$ oxidase complexed with an antibody FV fragment. Proc. Natl. Acad. Sci. US A. 94, $10547-$ 10553.

Payton, M., Auty, R., Delgoda, R., Everett, M. \& Sim, E. (1999). Cloning and characterization of arylamine $\mathrm{N}$-acetyltransferase genes from Mycobacterium smegmatis and Mycobacterium tuberculosis: increased expression results in isoniazid resistance. J. Bacteriol. 181, 1343-1347.

Pierattelli, R., Banci, L., Eady, N. A., Bodiguel, J., Jones, J. N., Moody, P. C., Raven, E. L., Jamart-Gregoire, B. \& Brown, K. A. (2004). Enzyme-catalyzed mechanism of isoniazid activation in class I and class III peroxidases. J. Biol. Chem.279, 39000-39009.

Preston, C. M. \& Barrett, J. (1987). Heligmosomoides polygyrus: peroxidase activity. Exp. Parasitol. 64, 24-28.

Pretorius, G. S., van Helden, P. D., Sirgel, F., Eisenach, K. D. \& Victor, T. C. (1995). Mutations in $k a t G$ gene sequences in isoniazid-resistant clinical isolates of Mycobacterium tuberculosis are rare. Antimicrob. Agents. Chemother. 39, 22762281 .

Ramaswamy, S. \& Musser, J. M. (1998). Molecular genetic basis of antimicrobial agent resistance in Mycobacterium tuberculosis: 1998 update. Tuber. Lung Dis. 79, 329.

Rawat, R., Whitty, A. \& Tonge, P. J. (2003). The isoniazid-NAD adduct is a slow, tight-binding inhibitor of InhA, the Mycobacterium tuberculosis enoyl reductase: adduct affinity and drug resistance. Proc. Natl. Acad. Sci. US A. 100, 13881-13886.

Regelsberger, G., Jakopitsch, C., Engleder, M., Ruker, F., Peschek, G. A. \& Obinger, C. (1999). Spectral and kinetic studies of the oxidation of monosubstituted phenols and anilines by recombinant Synechocystis catalase-peroxidase compound I. Biochemistry. 38, 10480-10488. 
Regelsberger, G., Jakopitsch, C., Ruker, F., Krois, D., Peschek, G. A. \& Obinger, C. (2000). Effect of distal cavity mutations on the formation of compound I in catalase-peroxidases. J. Biol. Chem. 275, 22854-22861.

Regelsberger, G., Jakopitsch, C., Furtmuller, P. G., Rueker, F., Switala, J., Loewen, P. C. \& Obinger, C. (2001). The role of distal tryptophan in the bifunctional activity of catalase-peroxidases. Biochem. Soc. Trans. 29, 99-105.

Rice-Evans, C. \& Baysal, E. (1987). Iron-mediated oxidative stress in erythrocytes. Biochem. J. 244, 191-196.

Rice-Evans, P. \& Rao, K. U. (1988). Evidence for positronium formation assisted by molecular recoil at a graphite surface covered with a semilayer of methane. Physical Review Letters 61, 581-584.

Ristow, M., Mohlig, M., Rifai, M., Schatz, H., Feldmann, K. \& Pfeiffer, A. (1995). New isoniazid/ethionamide resistance gene mutation and screening for multidrug-resistant Mycobacterium tuberculosis strains. Lancet 346, 502-503.

Ro, Y. T., Lee, H. I., Kim, E. J., Koo, J. H., Kim, E. \& Kim, Y. M. (2003). Purification, characterization, and physiological response of a catalase-peroxidase in Mycobacterium sp. strain JC1 DSM 3803 grown on methanol. FEMS Microbiol. Lett. 226, 397-403.

Rocha, E. R., Owens, G., Jr. \& Smith, C. J. (2000). The redox-sensitive transcriptional activator OxyR regulates the peroxide response regulon in the obligate anaerobe Bacteroides fragilis. J. Bacteriol. 182, 5059-5069.

Rouse, D. A. \& Morris, S. L. (1995). Molecular mechanisms of isoniazid resistance in Mycobacterium tuberculosis and Mycobacterium bovis. Infect. Immun. 63, $1427-$ 1433 .

Rouse, D. A., DeVito, J. A., Li, Z., Byer, H. \& Morris, S. L. (1996). Site-directed mutagenesis of the kat $G$ gene of Mycobacterium tuberculosis: effects on catalaseperoxidase activities and isoniazid resistance. Mol. Microbiol. 22, 583-592.

Rozwarski, D. A., Grant, G. A., Barton, D. H., Jacobs, W. R., Jr. \& Sacchettini, J. C. (1998). Modification of the NADH of the isoniazid target (InhA) from Mycobacterium tuberculosis. Science. 279, 98-102.

Saint-Joanis, B., Souchon, H., Wilming, M., Johnsson, K., Alzari, P. M. \& Cole, S. T. (1999). Use of site-directed mutagenesis to probe the structure, function and isoniazid activation of the catalase/peroxidase, $\mathrm{KatG}$, from Mycobacterium tuberculosis. Biochem. J. 338 ( Pt 3), 753-760. 
Sambrook, J., Fritsch, E.F., and Maniatis, T. (1989) Molecular Cloning : A Laboratory Manual. Cold Spring Harbour Laboratory. Cold Spring Harbour Press. New York.

Sanger, F., Nicklen, S. \& Coulson, A. R. (1977). DNA sequencing with chainterminating inhibitors. Proc. Natl. Acad. Sci. US A. 74, 5463-5467.

Santoni, E., Jakopitsch, C., Obinger, C. \& Smulevich, G. (2004a). Comparison between catalase-peroxidase and cytochrome $c$ peroxidase. The role of the hydrogenbond networks for protein stability and catalysis. Biochemistry. 43, 5792-5802.

Santoni, E., Jakopitsch, C., Obinger, C. \& Smulevich, G. (2004b). Manipulating the covalent link between distal side tryptophan, tyrosine, and methionine in catalaseperoxidases: an electronic absorption and resonance Raman study. Biopolymers 74, 46-50.

Schellhorn, H. E. \& Stones, V. L. (1992). Regulation of kat $F$ and katE in Escherichia coli $\mathrm{K}-12$ by weak acids. J. Bacteriol. 174, 4769-4776.

Schopf, J. W. (1993). Microfossils of the Early Archean Apex chert: new evidence of the antiquity of life. Science. 260, 640-646.

Sevinc, M. S., Ens, W. \& Loewen, P. C. (1995). The cysteines of catalase HPII of Escherichia coli, including Cys 438 which is blocked, do not have a catalytic role. Eur. J. Biochem. / FEBS 230, 127-132.

Sevinc, M. S., Switala, J., Bravo, J., Fita, I. \& Loewen, P. C. (1998). Truncation and heme pocket mutations reduce production of functional catalase HPII in Escherichia coli. Protein. Eng. 11, 549-555.

Sevinc, M. S., Mate, M. J., Switala, J., Fita, I. \& Loewen, P. C. (1999). Role of the lateral channel in catalase HPII of Escherichia coli. Protein Sci 8, 490-498.

Sharp, K. H., Moody, P. C., Brown, K. A. \& Raven, E. L. (2004). Crystal structure of the ascorbate peroxidase-salicylhydroxamic acid complex. Biochemistry. 43, 86448651 .

Sherman, D. R., Sabo, P. J., Hickey, M. J., Arain, T. M., Mahairas, G. G., Yuan, Y., Barry, C. E., 3rd \& Stover, C. K. (1995). Disparate responses to oxidative stress in saprophytic and pathogenic mycobacteria. Proc. Natl. Acad. Sci. USA. 92,66256629.

Shi, W., Hoganson, C. W., Espe, M., Bender, C. J., Babcock, G. T., Palmer, G., Kulmacz, R. J. \& Tsai, A. (2000). Electron paramagnetic resonance and electron nuclear double resonance spectroscopic identification and characterization of the tyrosyl radicals in prostaglandin $\mathrm{H}$ synthase 1 . Biochemistry. 39, 4112-4121. 
Sies, H. (1991). Oxidative stress: from basic research to clinical application. The American journal of medicine 91,31 S-38S.

Sies, H. (1993). Strategies of antioxidant defense. Eur. J. Biochem. / FEBS 215, $213-$ 219.

Singh, R., Wiseman, B., Deemagarn, T., Donald, L. J., Duckworth, H. W., Carpena, X., Fita, I. \& Loewen, P. C. (2004). Catalase-peroxidases (KatG) exhibit NADH oxidase activity. J. Biol. Chem.279, 43098-43106.

Sivaraja, M., Goodin, D. B., Smith, M. \& Hoffman, B. M. (1989). Identification by ENDOR of Trp191 as the free-radical site in cytochrome $c$ peroxidase compound ES. Science. 245, 738-740.

Smirnoff, N. \& Pallanca, J. E. (1996). Ascorbate metabolism in relation to oxidative stress. Biochem. Soc. Trans. 24, 472-478.

Smith, A. T., Santama, N., Dacey, S., Edwards, M., Bray, R. C., Thorneley, R. N. \& Burke, J. F. (1990). Expression of a synthetic gene for horseradish peroxidase C in Escherichia coli and folding and activation of the recombinant enzyme with $\mathrm{Ca}^{2+}$ and heme. J. Biol. Chem.265, 13335-13343.

Smith, A. T. \& Veitch, N. C. (1998). Substrate binding and catalysis in heme peroxidases. Current opinion in chemical biology 2, 269-278.

Smulevich, G., Jakopitsch, C., Droghetti, E. \& Obinger, C. (2006). Probing the structure and bifunctionality of catalase-peroxidase (KatG). J. Inorg Biochem. 100, 568-585.

Stadtman, T. C. (1991). Biosynthesis and function of selenocysteine-containing enzymes. J. Biol. Chem.266, 16257-16260.

Stehle, T., Ahmed, S. A., Claiborne, A. \& Schulz, G. E. (1991). Structure of $\mathrm{NADH}$ peroxidase from Streptococcus faecalis $10 \mathrm{C} 1$ refined at $2.16 \AA$ resolution. $J$. Mol. Biol. 221, 1325-1344.

Stoeckle, M. Y., Guan, L., Riegler, N., Weitzman, I., Kreiswirth, B., Kornblum, J., Laraque, F. \& Riley, L. W. (1993). Catalase-peroxidase gene sequences in isoniazid-sensitive and -resistant strains of Mycobacterium tuberculosis from New York City. J. Infect. Dis. s 168, 1063-1065.

Su, W. J., Lee, P. Y., Yu, K. W. \& Perng, R. P. (1997). Drug resistance of Mycobacterium tuberculosis isolated from patients at a medical center in Taiwan. Chinese medical journal 60, 21-27. 
Subsin, B., Thomas, M. S., Katzenmeier, G., Shaw, J. G., Tungpradabkul, S. \& Kunakorn, M. (2003). Role of the stationary growth phase sigma factor RpoS of Burkholderia pseudomallei in response to physiological stress conditions. $J$. Bacteriol. 185, 7008-7014.

Switala, J., Triggs-Raine, B. L. \& Loewen, P. C. (1990). Homology among bacterial catalase genes. Can. J. Microbiol. 36, 728-731.

Switala, J., O'Neil, J. O. \& Loewen, P. C. (1999). Catalase HPII from Escherichia coli exhibits enhanced resistance to denaturation. Biochemistry. 38, 3895-3901.

Switala, J. \& Loewen, P. C. (2002). Diversity of properties among catalases. Arch. Biochem. Biophys. 401, 145-154.

Touati, D., Jacques, M., Tardat, B., Bouchard, L. \& Despied, S. (1995). Lethal oxidative damage and mutagenesis are generated by iron in delta fur mutants of Escherichia coli: protective role of superoxide dismutase. J. Bacteriol. 177, 2305 2314.

Triggs-Raine, B. L. \& Loewen, P. C. (1987). Physical characterization of kat $G$, encoding catalase HPI of Escherichia coli. Gene. 52, 121-128.

Triggs-Raine, B. L., Doble, B. W., Mulvey, M. R., Sorby, P. A. \& Loewen, P. C. (1988). Nucleotide sequence of $k a t G$, encoding catalase HPI of Escherichia coli. $J$. Bacteriol. 170, 4415-4419.

Tsai, A., Wu, G., Palmer, G., Bambai, B., Koehn, J. A., Marshall, P. J. \& Kulmacz, R. J. (1999). Rapid kinetics of tyrosyl radical formation and heme redox state changes in prostaglandin H synthase-1 and -2. J. Biol. Chem.274, 21695-21700.

Tsukamoto, K., Itakura, H., Sato, K., Fukuyama, K., Miura, S., Takahashi, S., Ikezawa, H. \& Hosoya, T. (1999). Binding of salicylhydroxamic acid and several aromatic donor molecules to Arthromyces ramosus peroxidase, investigated by $\mathrm{X}$-ray crystallography, optical difference spectroscopy, NMR relaxation, molecular dynamics, and kinetics. Biochemistry. 38, 12558-12568.

Ursini, F., Maiorino, M., Brigelius-Flohe, R., Aumann, K. D., Roveri, A., Schomburg, D. \& Flohe, L. (1995). Diversity of glutathione peroxidases. Methods Enzymol. 252, 38-53.

Vainshtein, B. K., Melik-Adamyan, W. R., Barynin, V. V., Vagin, A. A. \& Grebenko, A. I. (1981). Three-dimensional structure of the enzyme catalase. Nature. 293, 411-412.

Vainshtein, B. K., Melik-Adamyan, W. R., Barynin, V. V., Vagin, A. A., Grebenko, A. I., Borisov, V. V., Bartels, K. S., Fita, I. \& Rossmann, M. G. 
(1986). Three-dimensional structure of catalase from Penicillium vitale at 2.0 Åresolution. J. Mol. Biol. 188, 49-61.

Victor, T. C., Pretorius, G. S., Felix, J. V., Jordaan, A. M., van Helden, P. D. \& Eisenach, K. D. (1996). kat $G$ mutations in isoniazid-resistant strains of Mycobacterium tuberculosis are not infrequent. Antimicrob. Agents. Chemother. 40, 1572.

Visick, J. E. \& Clarke, S. (1997). RpoS- and OxyR-independent induction of HPI catalase at stationary phase in Escherichia coli and identification of rpoS mutations in common laboratory strains. J. Bacteriol. 179, 4158-4163.

Vojtechovsky, J., Chu, K., Berendzen, J., Sweet, R. M. \& Schlichting, I. (1999). Crystal structures of myoglobin-ligand complexes at near-atomic resolution. Biophys.77, 2153-2174.

von Ossowski, I., Mulvey, M. R., Leco, P. A., Borys, A. \& Loewen, P. C. (1991). Nucleotide sequence of Escherichia coli katE, which encodes catalase HPII. $J$. Bacteriol. 173, 514-520.

Wada, K., Tada, T., Nakamura, Y., Kinoshita, T., Tamoi, M., Shigeoka, S. \& Nishimura, K. (2002). Crystallization and preliminary X-ray diffraction studies of catalase-peroxidase from Synechococcus PCC 7942. Acta. Crystallogr. D. Biol. Crystallogr. 58, 157-159.

Wang, J. Y., Burger, R. M. \& Drlica, K. (1998). Role of superoxide in catalaseperoxidase-mediated isoniazid action against mycobacteria. Antimicrob. Agents. Chemother. 42, 709-711.

Weber, K., Pringle, J. R. \& Osborn, M. (1972). Measurement of molecular weights by electrophoresis on SDS-acrylamide gel. Methods Enzymol. 26 PtC, 3-27.

Welinder, K. G. (1991). Bacterial catalase-peroxidases are gene duplicated members of the plant peroxidase superfamily. Biochim. Biophys. Acta. 1080, 215-220.

Welinder, K. G., Mauro, J. M. \& Norskov-Lauritsen, L. (1992). Structure of plant and fungal peroxidases. Biochem. Soc. Trans. 20, 337-340.

Wengenack, N. L., Uhl, J. R., St Amand, A. L., Tomlinson, A. J., Benson, L. M., Naylor, S., Kline, B. C., Cockerill, F. R., 3rd \& Rusnak, F. (1997). Recombinant Mycobacterium tuberculosis $\mathrm{KatG}(\mathrm{S} 315 \mathrm{~T})$ is a competent catalase-peroxidase with reduced activity toward isoniazid. J. Infect. Dis. 176, 722-727.

Wilming, M. \& Johnsson, K. (1999). Spontaneous Formation of the Bioactive Form of the Tuberculosis Drug Isoniazid. Angew. Chem. Int. Ed. Engl. 38, 2588-2590. 
Wilming, M. \& Johnsson, K. (2001). Inter- and intramolecular domain interactions of the catalase-peroxidase $\mathrm{KatG}$ from M. tuberculosis. FEBS Lett. 509, 272-276.

Wood, P. M. (1988). The potential diagram for oxygen at $\mathrm{pH}$ 7. Biochem. J. 253, 287-289.

Xia, R., Webb, J. A., Gnall, L. L., Cutler, K. \& Abramson, J. J. (2003). Skeletal muscle sarcoplasmic reticulum contains a NADH-dependent oxidase that generates superoxide. Am. J. Physiol. Cell. Physiol. 285, C215-221.

Yamada, Y., Saijo, S., Sato, T., Igarashi, N., Usui, H., Fujiwara, T. \& Tanaka, N. (2001). Crystallization and preliminary X-ray analysis of catalase-peroxidase from the halophilic archaeon Haloarcula marismortui. Acta. Crystallogr. D. Biol. Crystallogr. 57, 1157-1158.

Yokota, K. \& Yamazaki, I. (1965). Reaction of peroxidase with reduced nicotinamide-adenine dinucleotide and reduced nicotinamide-adenine dinucleotide phosphate. Biochim. Biophys. Acta. 105, 301-312.

Yokota, K. \& Yamazaki, I. (1977). Analysis and computer simulation of aerobic oxidation of reduced nicotinamide adenine dinucleotide catalyzed by horseradish peroxidase. Biochemistry. 16, 1913-1920.

Yoshikawa, S. (1997). Beef heart cytochrome $c$ oxidase. Current opinion in structural biology 7, 574-579.

Youatt, W. G. \& Fay, L. D. (1959). Experimental brucellosis in whitetailed deer. Am. J. Vet. Res. 20, 925-926.

Young, T. A., Delagoutte, B., Endrizzi, J. A., Falick, A. M. \& Alber, T. (2003). Structure of Mycobacterium tuberculosis $\mathrm{PknB}$ supports a universal activation mechanism for Ser/Thr protein kinases. Nat. Struct. Biol. 10, 168-174.

Yu, S., Girotto, S., Lee, C. \& Magliozzo, R. S. (2003). Reduced affinity for Isoniazid in the S315T mutant of Mycobacterium tuberculosis KatG is a key factor in antibiotic resistance. J. Biol. Chem.278, 14769-14775.

Zamocky, M., Janecek, S. \& Koller, F. (2000). Common phylogeny of catalaseperoxidases and ascorbate peroxidases. Gene. 256, 169-182.

Zamocky, M., Regelsberger, G., Jakopitsch, C. \& Obinger, C. (2001). The molecular peculiarities of catalase-peroxidases. FEBS Lett. 492, 177-182.

Zamocky, M. (2004). Phylogenetic relationships in class I of the superfamily of bacterial, fungal, and plant peroxidases. Eur. J. Biochem. 271, 3297-3309. 
Zhang, Y., Heym, B., Allen, B., Young, D. \& Cole, S. (1992). The catalaseperoxidase gene and isoniazid resistance of Mycobacterium tuberculosis. Nature. 358, 591-593.

Zhang, Y. \& Young, D. B. (1993). Molecular mechanisms of isoniazid: a drug at the front line of tuberculosis control. Trends. Microbiol. 1, 109-113.

Zhao, X., Girotto, S., Yu, S. \& Magliozzo, R. S. (2004). Evidence for radical formation at Tyr-353 in Mycobacterium tuberculosis catalase-peroxidase (KatG). $J$. Biol. Chem.279, 7606-7612.

Zhao, X., Yu, H., Yu, S., Wang, F., Sacchettini, J. C. \& Magliozzo, R. S. (2006). Hydrogen peroxide-mediated isoniazid activation catalyzed by Mycobacterium tuberculosis catalase-peroxidase (KatG) and its S315T mutant. Biochemistry. 45, $4131-4140$.

Zheng, M., Aslund, F. \& Storz, G. (1998). Activation of the OxyR transcription factor by reversible disulfide bond formation. Science. 279, 1718-1721.

Zheng, M. \& Storz, G. (2000). Redox sensing by prokaryotic transcription factors. Biochem. Pharmacol. 59, 1-6.

Zoldak, G., Sut'ak, R., Antalik, M., Sprinzl, M. \& Sedlak, E. (2003). Role of conformational flexibility for enzymatic activity in NADH oxidase from Thermus thermophilus. Eur. J. Biochem. / FEBS 270, 4887-4897.

Zou, P., Borovok, I., Ortiz de Orue Lucana, D., Muller, D. \& Schrempf, H. (1999). The mycelium-associated Streptomyces reticuli catalase-peroxidase, its gene and regulation by FurS. Microbiology 145 ( Pt 3), 549-559. 